\title{
Flow-induced arterial remodeling : role of angiotensin Il and bradykinin
}

Citation for published version (APA):

Hilgers, R. H. P. (2004). Flow-induced arterial remodeling : role of angiotensin II and bradykinin. [Doctoral Thesis, Maastricht University]. Datawyse / Universitaire Pers Maastricht. https://doi.org/10.26481/dis.20040625rh

Document status and date:

Published: 01/01/2004

DOI:

10.26481/dis.20040625rh

Document Version:

Publisher's PDF, also known as Version of record

\section{Please check the document version of this publication:}

- A submitted manuscript is the version of the article upon submission and before peer-review. There can be important differences between the submitted version and the official published version of record.

People interested in the research are advised to contact the author for the final version of the publication, or visit the DOI to the publisher's website.

- The final author version and the galley proof are versions of the publication after peer review.

- The final published version features the final layout of the paper including the volume, issue and page numbers.

Link to publication

\footnotetext{
General rights rights.

- You may freely distribute the URL identifying the publication in the public portal. please follow below link for the End User Agreement:

www.umlib.nl/taverne-license

Take down policy

If you believe that this document breaches copyright please contact us at:

repository@maastrichtuniversity.nl

providing details and we will investigate your claim.
}

Copyright and moral rights for the publications made accessible in the public portal are retained by the authors and/or other copyright owners and it is a condition of accessing publications that users recognise and abide by the legal requirements associated with these

- Users may download and print one copy of any publication from the public portal for the purpose of private study or research.

- You may not further distribute the material or use it for any profit-making activity or commercial gain

If the publication is distributed under the terms of Article $25 \mathrm{fa}$ of the Dutch Copyright Act, indicated by the "Taverne" license above, 
Flow-Induced Arterial Remodeling:

Role of Angiotensin II and Bradykinin 
copytight Rob Higcrs, Maastricht 2004

ISBN 9052784132

Printed by: Datawyse | Universitaire Pers Mastricht

Cover designed by: Rob Itilgers | Gregorio Pazzi

Description cover: two photon real-time microscopy image of a cannulated uterine artery.

Cell nucle stained in green, elastin fibers in red. Image kindly provided by:

Remco Megens/Mare wan Zandwoort/Wim Engels/Dick Slaaf. 


\title{
Flow-Induced Arterial Remodeling
}

\section{Role of Angiotensin II and Bradykinin}

\author{
PROEFSCHRIFT \\ Ter verkrijging van de graad wan doctor \\ aan de Universiteit van Maastricht, \\ op gezag van Rector Magnificus, \\ Prof. Mr. C.P.M.F. Mols, \\ volgens het besturur van College van Decanen, \\ in het openbaar te verdedigen \\ op vrijclag 25 juni 2004 om 12:00 uur \\ door \\ Robertus Hubertus Petronella Hilgets \\ geboren op 7 augustus 1972 \\ te Kerkrade
}

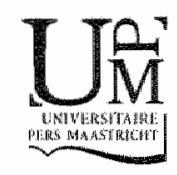


Promotor:

Prof. dr. J.G.R. De Mey

Coprostritor:

Dr. P.M.H. Schiffers

Beoordelingscommissie:

Prof. dr. M.J. Post (voorzitter)

Prof. dr. in. A.P.G. Hoeks

D. L.L.H. Peeters

Prof. dr. JJ. Piek (AMC, Amsterdam)

Dr. J.L. Waltenberger

Financial support by the Netherlands Heart Foundation for the publication of this thesis is gratefully acknowledged.

Additional support was granted by: Living Systems Instrumentation (USA). 


\section{Table of Contents}

\section{CHAPTER 1 Introduction 7}

CHAPTER 2 Flow-Dependent Dilatation Mediated by Endogenous Kinins Requites Angiotensin A $\mathrm{T}_{2}$ Receptors. Cir Rer. In Pres 29

CHAPTER 3 Role of Tissue Kallikrein During Arterial Remodeling in Response to Altered Blood. SAbmitted. 41

CHAPTER 4 Uterine Attery Structural and Functional Changes During Pregnancy in Tissue Kallikrein-Deficient Mice. $A T T$ B. 2003;23-1826-1832. 51

CHAPTER 5 Intra-Arterial Bradykinin and Vasodilatacion in relation to Acute and Chronic Changes in Blood Flow. Swownited. 67

CHAPTER 6 Changes in Vascular Distensibility During Angiotensin-Converting Enzyme Inhibition Inwolve Bradykinin Type 2 Receptors. 7 Varc Res. 2004,41:78-27. 77

CHAPTER 7 Tissue Angiotensin-Converting Enzyme in Imposed and Physiological Flow-Related Arterial Remodeling in Mice. ATT B. 2004,892-897. 91

CHAPTER 8 Utentine Artery Dilatation and Remodeling During Postponed Murine Pregnancy. Submitted. 105

CHAPTER 9 Review article: Acute and Chronic Adaptive Responses to Altered Blood Flow in Latge and Small Arteries. Swontited. 117

CHAPTER 10 General Discussion. 139

REFERENCES 145

SUMMARY 171

SAMENVATTTNG 175

DANKWOORD 179

CURRTCULUM VITAE 181

GRANTS AND AWARDS 183

LIST OF PUBLISHED ARTICLES 183

LIST OF SUBMITTED ARTTCIES 183

LIST OF ABSTRACTS 184 

Chapter 1

\section{Introduction}




\section{General Introduction}

Blood vessels are permanently subjected to mechanical forces. The pulsatile nature of arterial blood flow affects the inner layer of the arterial wall, the endothelium, which senses the frictional dragging force (fluid shear stress) of the blood flow. Transmural pressure causes tangential forces (circumferential wall stress) that affect the cellular and exrracellular matrix components of all three layers of the arterial wall (the turicae intima, media and adventitia)(Figure 1.1).

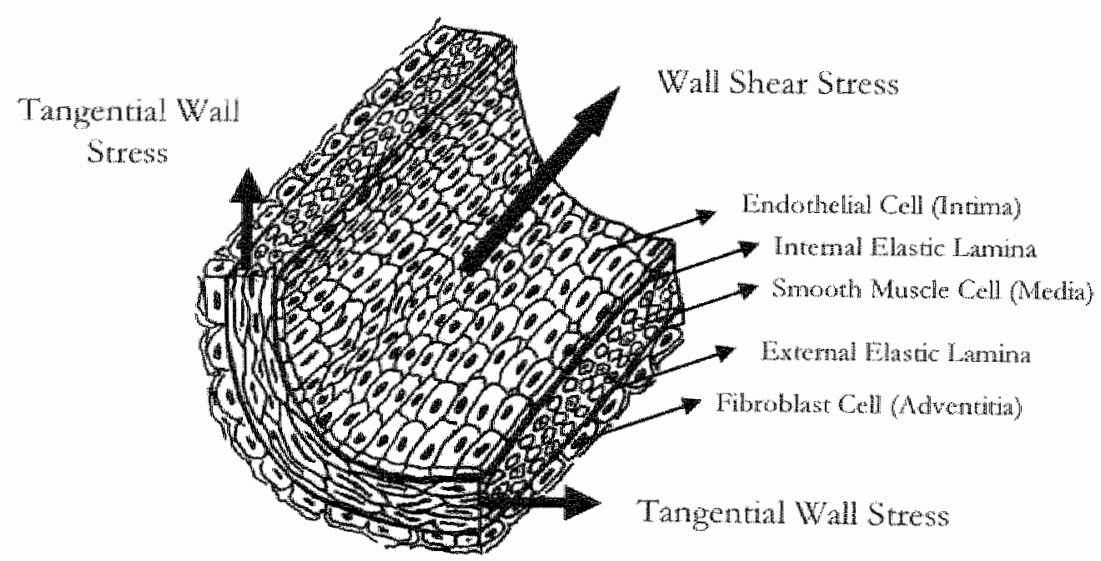

Figute 1.1. Simplifed model of the arterial wall components and the hemodynamic forcas which they are

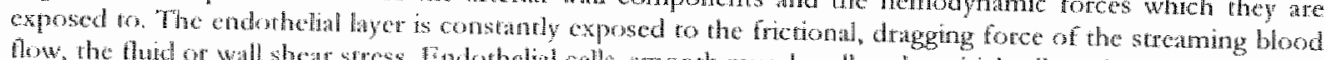

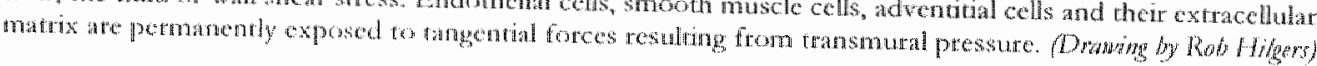

Acute changes in pressure and flow result in transient adjustments of the vascular lumen diameter, mediated by myogenic contractile responses or release of paracrine vasoactive compounds that aim to restore shear stress and circumferential wall stress. For instance, an increase in blood pressute causes a narrowing or constriction of the artery (myogenic rone), while a rise in arterial blood flow results in a widening or dilatation of the vessel (flow-induced dilatation).

When these mechanical forces persist for a longer period of time they may cause structural alterations in arterial wall shape, composition and function. This arterial tremodeling describes the transformation of the artery (diameter and wall mass changes) in response to chronically altered mechanical forces. It participates in the pathogenesis of many cardiovasculat diseases and complications. For example, hypertension is 
accompanied by increased wall thickening in small resistance arteries and arterioles resulting in smallet lumen diameters. Atherosclerosis develops at sites that experience reduced or turbulent wall shear stresses, especially near bifurcations of large arteries such as the aorta or carotid atrery.

Unfortunately, cardiovascular diseases such as hypertension, atherosclerosis, precclampsia, myocatdial infatction, and heart fallure remain the number one cause of death in western societies. Research on vascular temodeling that ams to univel the mechanisms and mediators involved in the onset of cardiovascular complications is necessary for the development of new therapeutic strategies for the treatment of these diseases.

\section{Acute Arterial Responses to Mechanical Stimuli}

\section{Pressure and Myogenic Tone}

Vascular smooth muscle cells (VSMCs) are particularly sensitive to stretch and stresses and transmit these mechanical forces into biochemical signals. Intraluminal pressure results in a tangential stress upon the arterial wall or circumferential wall stress (CWS). It is defined by the law of Laplace:

$$
C W S=\frac{T P \cdot r}{W t}
$$

where TP is transmural pressure (in $\mathrm{N} / \mathrm{m}$ ), $r$ is the vasculat inner or lumen tadius (in mm) and $W t$ is wall thickness (in mm). Small atteries $(\varnothing<300 \mu \mathrm{m}$ ) constrict in response to an elevation of pressure, reducing their radius and slightly incteasing their wall thickness, hence restoring wall stress toward normal levels. This pressure-induced vasoconstriction is known as the myogenic tone or the myogenic response. 150 increases with decreasing vessel diameter, ${ }^{115}$ although the cetebtal wascular bed ${ }^{404}$ may be an exception to this nule. Myogenic tone can casily be investigated in vitro. When small resistance arterics of arterioles are isolated, cannulated and incubated in a physiological salt solution, raising the intraluminal pressure will result in a pressure-dependent constriction (active, Figure 1.2) which stays stable over a broad pressure range. Withdrawal of $\mathrm{Ca}^{2+}$ ions from the incubate and administration of vasodilator agonis abolishes this myogenic tone and fully relaxes the VSMCs so that the arterial diameter contimues to increase und a plateau is reached (passive, Figure 1.2).

The myogenic response is mediated by a non-neuralts and endotheliumindependent mechanism. It enables small arteries such as arterioles to (1) autoregulate local blood flow, (2) establish basal vascular tone and peripheral resistanco, and (3) regulate capillary hydrostatic pressure. ${ }^{116231}$ It has long been appreciated that $\mathrm{Ca}^{2}$ ions play a pivotal role in smooth muscle contraction and in the setting of atteriolar tone. "This effect of $\mathrm{Ca}^{2+}$ is assumed to be mediated through $\mathrm{Ca}^{2+}$-calmodulin activation of myosin light chain kinase. The intracellulat signaling mechanisms of this response remain focus of extensive rescatch, but it is agteed upon that a myogenc tone is initiated by VSMC 
depolarizarion, which then regulates $\mathrm{Ca}^{2+}$ entry through voltage-gated $\mathrm{Ca}^{2+}$ channels ${ }^{3}$ ? and stretch-sensitive ion channels. "16 For an overview of the medators involved in myogenic tone the reader is teferred to more elaborate review aruicles. 17,005

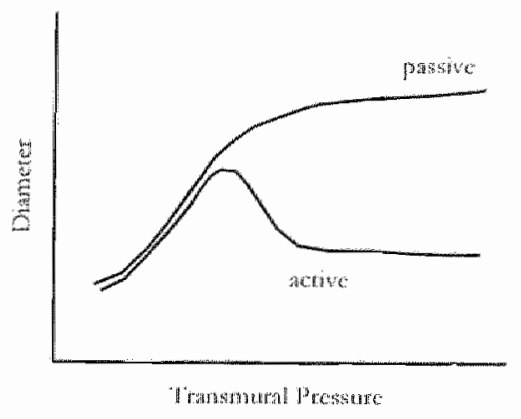

Figure 1.2. Example of mygenic tone measurement in an isolated small artery. An arterial segment $(3 \mathrm{~mm})$ is cannulated beween two small glass pipettes and incubarcd in a physological salt solution withour $\mathrm{Ca}^{2}$

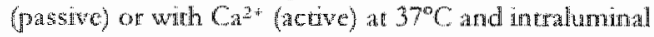
pressure is raised in small seps (to. $10 \mathrm{~mm} / \mathrm{m}$ ) and the diameter is recorded funct at stable dianeter is rached). "Transmunal pressure is plonted aganst flameter (Pressure-Dianeter curve).

\section{Filow (Shear Stress)-Induced Vasodilatation}

Endothelial cells (ECs) are ditectly in contact with the streaming blood and are well adapted to sense the dragging, frictional force (shear stress) of the pulsatile blood flow. Wall shear stress (WSS) is, accotding to Pousseuille's law, defined by the formula:

$$
W S S=\frac{4 \cdot \eta \cdot Q}{\pi \cdot r^{3}}
$$

in which $\eta$ is the blood viscocity (in dyne.s.cm $\left.{ }^{7}\right), Q$ is the blood flow relocity (in $\mathrm{cm}^{3} . \mathrm{s}^{-1}$ ), and, is the radius of the arterial lumen (in $\mathrm{cm}$ ). Nomal steady laminar shear stress stimulates celluat tesponses that are essential for nomal endothelial cell function, such as tegulating pemeability of plasma lipoproteins, adhesion of leukocytes, enabling balance between anti-and prothromboric factors, telease of endothelium-derived growth factors and vasoactive agents ${ }^{\text {thi }}$ and mainfaining an atheroprotective environment. ${ }^{\text {it4 }}$ Increases in blood fow, and thus shear stress, lead to release of endothelium-derived relaxing factors, such as nitric oxide and prostacyclin, that cause a diamcter enlargement, which tends to restore wall shear stress values toward notmal.

\section{Interation Between Myogentic Tane and Flon-Indwed I arodilatation}

A distinction and an interaction between pressure-induced and shear stress-induced arterial responses have been clearly demonstrated in an in wivo experiment by Pohl and colleagues. ${ }^{20} \mathrm{In}$ sits exposed and saline-perfused rabbit small mesenteric arteries wete exposed to an elevation of perfusion pressure, which increased both transmural pressure 
and flow. After an initial dilatation, the vessels developed a myogentic constriction, followed by a diatation, which was shear stress-dependent, since nonperfused vessels exposed to the same intraluminal pressure developed a myogentic response without a subsequent dilatation. ${ }^{426}$ These experiments indicate that myogenic responses can be opposed by flow-induced dilatations, suggesting a mechanism for maintaining adequate tissue perfusion during for instance exercise, when pressure and shear stress increase simulaneously. Also comperition between myogenic rone and How-induced dilatation occurs, since strong lewels of myogenic tone in cannulated piessurzed coronary arterioles attenuated flow-induced dilatation. ${ }^{302}$ This competition might be a mechanism to protect tissues from overperfision and increased permeability leading to edemas.

In some types of isolated arterial segments, flow can cause either a constriction on a dilatation, depending on the level of the pre-existing vasomotor tone and on the membrane potential of the VSMCs. ${ }^{44}$ Resting tone is proposed to be determined by the activity of ATP-sensitive 262 and calcium-dependent ${ }^{38}$ potassium channels, where a fraction of these channels is open, such that membrane potential has an intemediate value (approximately $-58 \mathrm{mV}$ ). The opening of potassium channels would lead to hypetpolarization $(-62 \mathrm{mV})$ and dilatation, whereas closure results in depolarization (-49) $\mathrm{m}$ V) and contraction.

\section{Medranisms of Mechawomatudution}

Evidence indicating a role of the endothelium as a shear stress-sensor emerged from is wiro studies of cultured endothelial monolayers 10,12 and isolated cannulated small arteries. 24827 In response to sustained steady flows, ECs align and clongate in the direction of the flow, ${ }^{110,255}$ and release vasoactive factors (ag nitric oxide, prostacyclin. endothelin-1). Confomational changes of proteins ate most likely involved. The specialized regions of the plasma membrane known as focal adbesion sites link cytoskeletal proteins to the extracellular matrix, and modulate cellular shape and motility. Shear stress leads to a rapid deformation and reorganization of actin-containing stress fibers, as well as othet cytoskeletal intermediare flaments, such as vimentin, at the sites of cell-matrix contacts foming the focal adhesion complexes (Figure 1,3 ). ${ }^{21}$ Integrins are important linkage proteins and are likely candidates for mechanotranduction in their capaciry to bind to the extracelluar matrix on the cell surface and to the cytosketetal proteins in the cytoplasm in Indeed, sheat stress modulates the binding affinity of integtins to the Arg Gly-Asp (RGD) sequence in integtin receptor proteins such as fibronectin. "The underlying rapid signal transduction pathways involve muldiple kinases such as focal adhesion kinase ( $F A K$ ) that activate downstream mitogen-activated protein kinases (MAPKs, \&.g. ERK , transcription factors such as muclear factor-KB, and receptor associated $G$ protems such as Rho. $17,42,4 \%$ Ultimaty, the responses to shear stress lead to the rapid phosphorylation and activation of enzymes, such as endothelial nitric oxide synthase (eNOS)(see next section). Othet vasoactive compounds can be released in response to flud shear stress as well. These endorhelium-derived factors include prostaglandines, ${ }^{4}$ bradykinin, ${ }^{37}$ and the still poorly characterized endorhclium-derived hyperpolarizing factor (EDHF), ${ }^{2+4}$ as well as vasoconstrictors, such as colothelin $1,{ }^{297}$ and reactive oxygen species. 324 


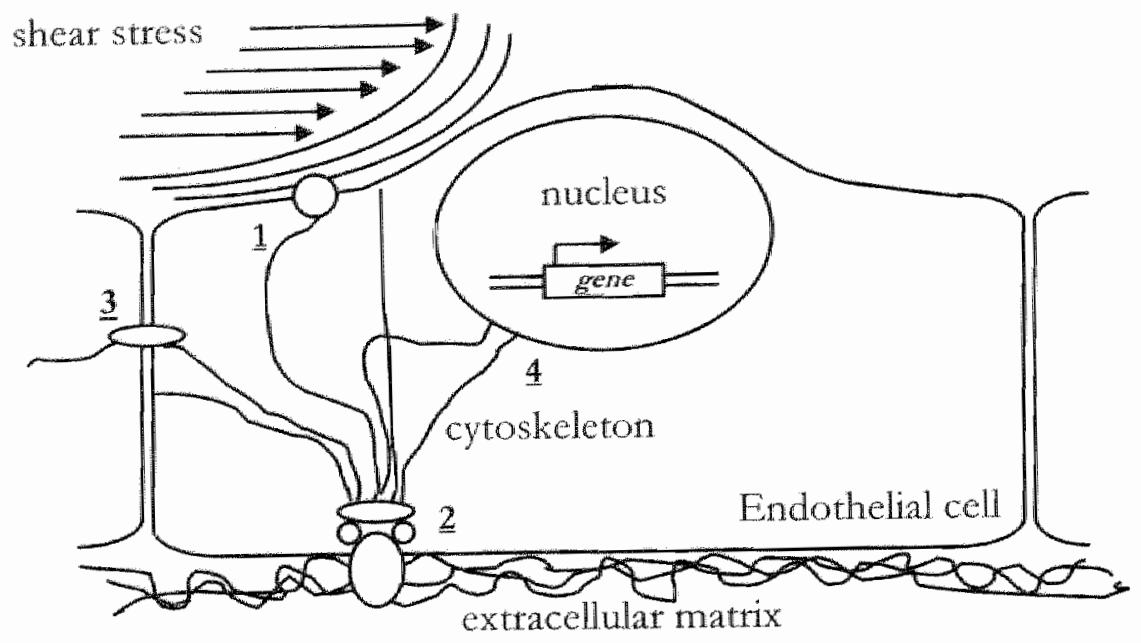

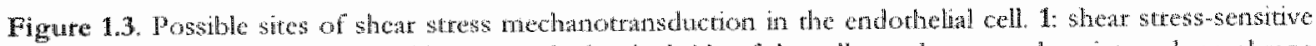
protens bound on the phospholid layer on the luminal side of the cell membrane, such as integral membrane

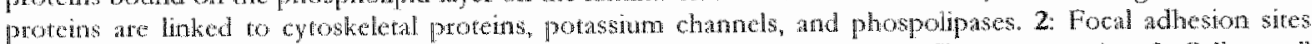

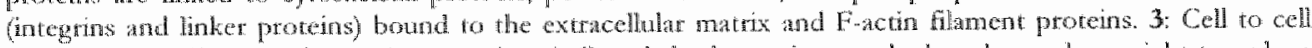

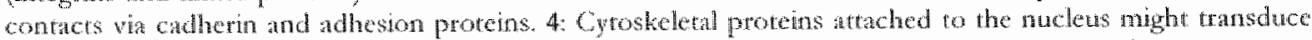

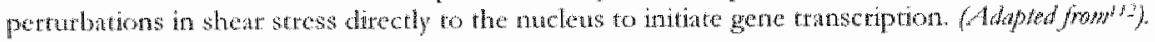

\section{Entothelial Nitric Oxide Synthase Actipation}

Numcrous studies have been performed to elucidate the mechanism of shear stressinduced activation of $\mathrm{NOSS}$ and NO release. In general, $\mathrm{NOS}$ (or NOS3) catalyzes the production of the vasodilator nitric oxide (NO) by the oxidation of L-arginine to Lcimuline. "The diatomic gas diffuses to the underlying VSMCS where it acrivates soluble guarylate cychase leading to cyclic GMP production and subsequent VSMC telaxation (for a review $\mathrm{sec}^{224}$ ). $\mathrm{eNOS}$ is targeted to caveolac (invaginations of the plasma membrane) by comanslational $\mathrm{N}$-mytristoylation and postranshational palmitoylation, ${ }^{174}$ and is held inactive wa binding with caveolin-1.24 An increase in intracellular $\mathrm{Ca}^{2+}$, following recepror-dependent and -independent agonist stimulation, enhances NO production and causes vasodilatation. ${ }^{154}$ Binding of calmodulin and associated proteins is essential for agonist-induced activation of eNOS, 7572 However, sheat stress activates eNOS via a $\mathrm{Ca}^{21}$-independent mechanism." Evidence that eNOS can be stimulated by at least two independent signaling pathways came from an observation that distuption of the cytoskclcton attenuated the flow-induced release of $\mathrm{NO}$, but did not affect the agonist-

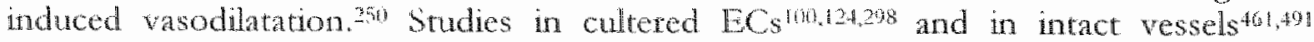
demonstrated the production of NO by flow via eNOS activation. Shear stress rapidly activates a signaling pathway involving several tyrosine kinases, $\mathrm{FAK}$, proline-rich tyrosine kinase (PYK2), and vascular endothelial. growth factor receptor 2 (Flk 
1/VEGFR2:268.321,59) Ultimately, this proximal tyrosine kinase signaling loads to the mote downstream signaling via phosphoinositide 3-kinase $(\mathrm{PI} ; \mathrm{K})$ and subsequent serine/threonine protein kinase Akt activation that phosphorylates eNOS at amino acid residue serine $1177,{ }^{124169}$ leading to activation of eNOS and the release of NO within seconds (Figure 1.4).

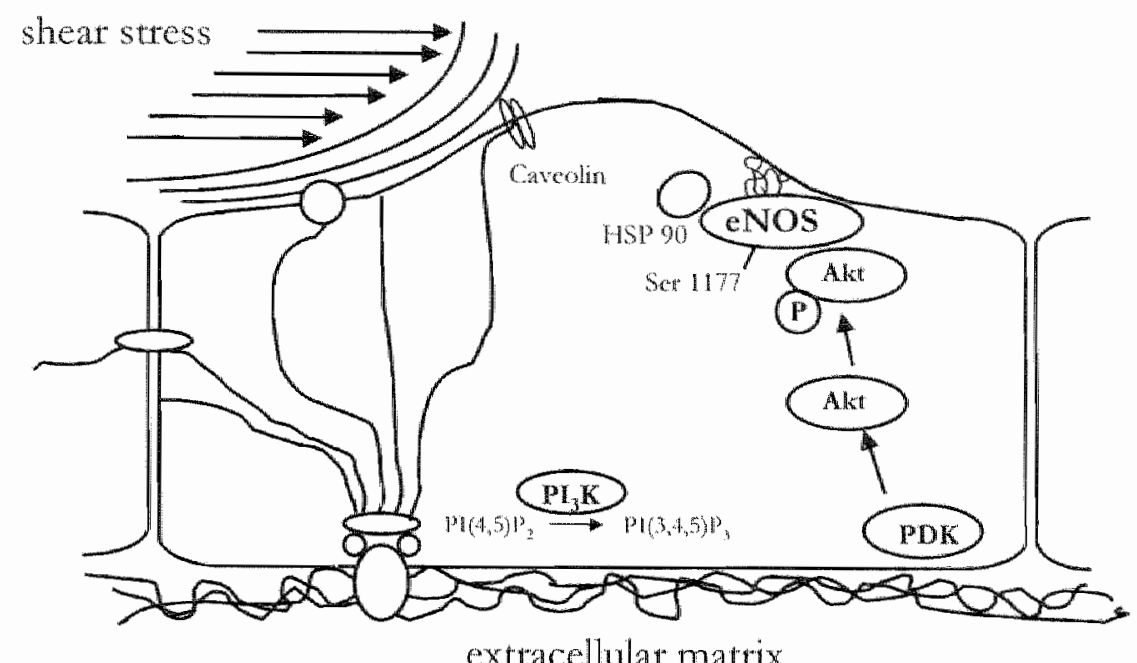

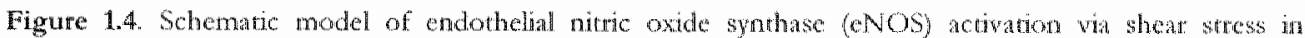
endothelint cells. Under physiological shear stress levels (resting condatons) low levels of No are produced

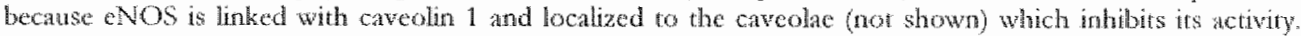

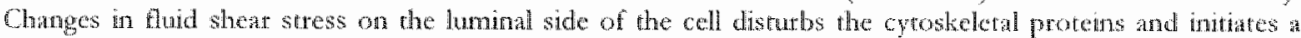

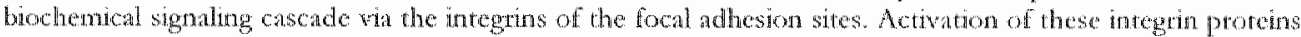

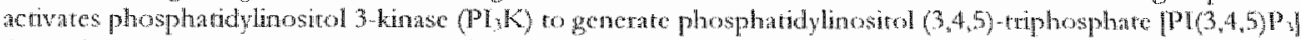

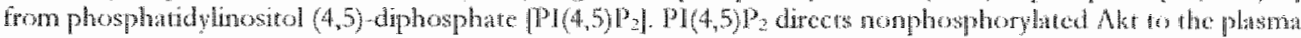

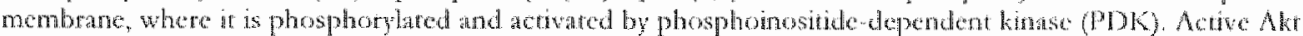

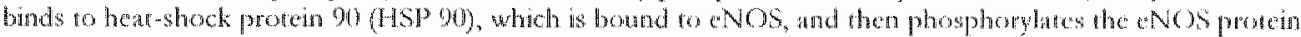

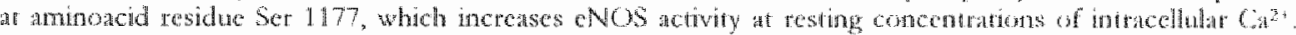

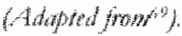

\section{Shear Stress and Arevial Gene Expression}

In contrast to the acute shear stress-induced signaling tesponses described in the previous section, more sustained $P 1$ hour) shear stress-induced responses involve regulation at the level of gene expression. A striking similarity of these shear stress-inducible genes is their atheroprotective role, indicating that nomal biood flow is vital for maintaining a healthy balance between a pro- and anticoagulant, inflammatory, -oxidant, and proliferative state, and that chronic nonphysiological shear seress values distupt this balance toward the onset of vascular diseases such as atherosclerosis. Many genes important in thrombosis and haemostasis, inflammation, cell proliferation, and extracellulat matrix temodeling are regulated by shear stress, including tissue plasminogen activator (TPA), ${ }^{123}$ monocyte chemotactic protein 1 (MC,P-1), ${ }^{\text {5in }}$ interccllular adhesion 


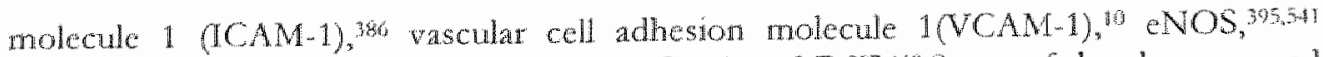

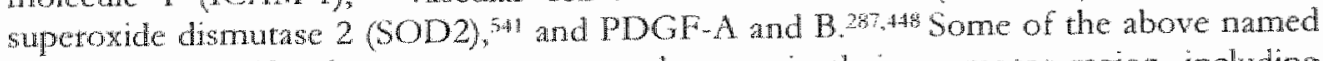
genes have specific shear stress-response dements in theit promotor region, including PDOF $A$ - and $B$, and $M C P-1223,46, A$ more indrect pathway of shear-induced changes in gene expression involves the NO/cGMP pathway, which was described above with respect to vasodilatation. $2{ }^{25}$

\section{Chronic Arterial Responses to Mechanical Stimuli}

\section{Pressure-Related Arterial Remodeling}

Hypertension, a vascular disease with an incidence of nearly $20 \%$ in the human population, affects all parts of the cardiovasculat system. Studying the vasomotor responses of small vessels exposed to high blood pressure enables better understanding of the underlying signaling events that lead to functional adaptations, which precede structural and more permanent arterial changes.

Since large vessels possess only minor vasomotor rone, theit capacity to acutely nomalize increases in hemodynamic forces is limited. As a resul, large arteries undergo structural remodcling (an increase in arterial wall thickness that nomalizes circumterental wall stress) carliet than smaller arteries do. In arterioles, increases in transmut pressure initially induce a constriction, which opposes the passive distension. During this myogenic response, the thickness of the arteriolar wall increases and circumferential wall stress is nomalized. Consequently, this hemodynamic sensor will no longer be activated and the proliferative signaling will not occur." Because of the rearangement of dynamic focaladhesion sices, by which the arteval cells are conmected to their matrix, the maintained constriction will ultimately become structural, leading to inward eutrophic remodeling. ${ }^{\text {? }}$ Smali arteries (D: $100-300 \mu \mathrm{m}$, possess a weak myogenic reactivity and consequenty display an intemediate rype of remodeling.

A general characteristic of hypertension is that peripheral vasculat resistance is increased cspocially in arrerioles and capillanes. 53,37 Besides ratefaction ( $\downarrow$ vessel number) and inware eutrophic temodeling, this is duc to an augmented myogenic response, ${ }^{24.3}$ vin chlanced release of constrictor factors such as codothelin 1 , th prostaglandin $\mathrm{H}$, and thromboxane $A_{2}{ }^{242}$ which incrase the $C^{2+4}$ sensivity of the contractile apparatus.

The colluar signaling cascades involved in VSMC profiferation are intricate and are mostly derived from cell culture experiments. However, recent infomation came from intact wessels, which were cantulated and pressurized in vito.35,5s5 Increased transmutal pressure leads to the release of angiorensin $11,2,318$ which wia the specific angiotensin $\Pi$ receptor subype $1(A T)$, activates phospholipase $C$ and protein kinase $C(P K C$ ) in tat VSMC.2Z PKC activates the Ras-Raf parhway, and MAPKs such as extraceliular signaltegulated kinase (ERK). ERK1/2 is a key enzyme in mediating the VSMC proliferative actions in response to angiotensin $\Pi 152$ and pressure 150,53985 ERK $1 / 2$ stimulates the expression of several proto-oncogenes such as $f \circ$ and $c$-my causing proliferation and proten expression necessary for impotant remodeling events. ${ }^{32}$ One of these processes is extracellutat matrix degudation in which matrix metalloproteinases (MMPs) especially the elastinolytic MMPs, such as MMP-2 (ope IV collagenase), MMP-9 and MMP-12 play 
a pivotal role. Proto-oncogenes of the AP-1 family such as for regulate MMP-9 promotor activity. ${ }^{102}$ Platelet-derived growth factor (PDGF)-A and angiotensin II are also expressed in response to an increase in pressure and appear to be critical for VSMC hypertrophy and lypetplasia through autocrine mechanisms. $40.35,384$

\section{Flow (Sbear Swors)-Related Antwal Remadeling}

Flow-related atterial remodeling is the process involving arterial adaptations to chronic altetations in flow, that aim to nomalize the imposed wall stresses on the endothelum and the arterial wall, via structural changes of the lumen diameter (WSS) and wall thickness (CWS). The arterial remodeling of an arterial bed in response to changes in blood flow can occur via two mechanisms: capillary network expansion via the sprouting of new capillaties from existing capillaties (angiogenesis) and enlargement of existing collateral arterioles (arteriogenesis). ${ }^{1325}$ Changes in architecture of the arterial wall in response to alterations in hemodynamic stmuli occut under physiological as well as under pathological conditions, but can also be experimentaly induced wia surgical manipulations outlined in the following sections.

Several remodeling responses can be distinguished (Figure 1.5). The classification that was proposed in 1996 for arterial structural responses in hypertension ${ }^{\text {was }}$ is based on the changes of the structural arrerial lumen diameter (inwatd or outward) and on changes in wall mass (increased: hypertrophic, decteased: hypothrophic, and unchanged. eutrophic). For example, an increase in lumen diameter without a change in medial crosssectional area, would be denoted as an outward eutrophic remodeling. "This remodeling classification can also be applied to flow-related arterial diameter and wall mass changes. Sustained elevated blood flow will eventually result in a structural luminal expansion, most likely from reatrangement of existing wall mass leading to a thinner wall. "This thinning of the arterial wall will increase circumferential wall stress, which triggers growth and proliferation of arterial wall cells. The resulting wall hypettrophy nomalizes this wall stress. $71,4,4$

Chronic reductions in arterial blood flow lead to a reduction in lumen diameter that normalizes wall shear stress. This narrowing of the lumen diameter is stuctural, since the diameter can not be restored to control walues after application of several vasodiator compounds 3 days postligation in mesenteric arteries 4.34 and 7 days postligation in carotid arteries. ${ }^{62,462}$ Reductions in arterial diameter decrease circumferential wall stress which not only induces hypotrophic signaling but also stimulates atrophy and apoptosis of the VSMCs." 


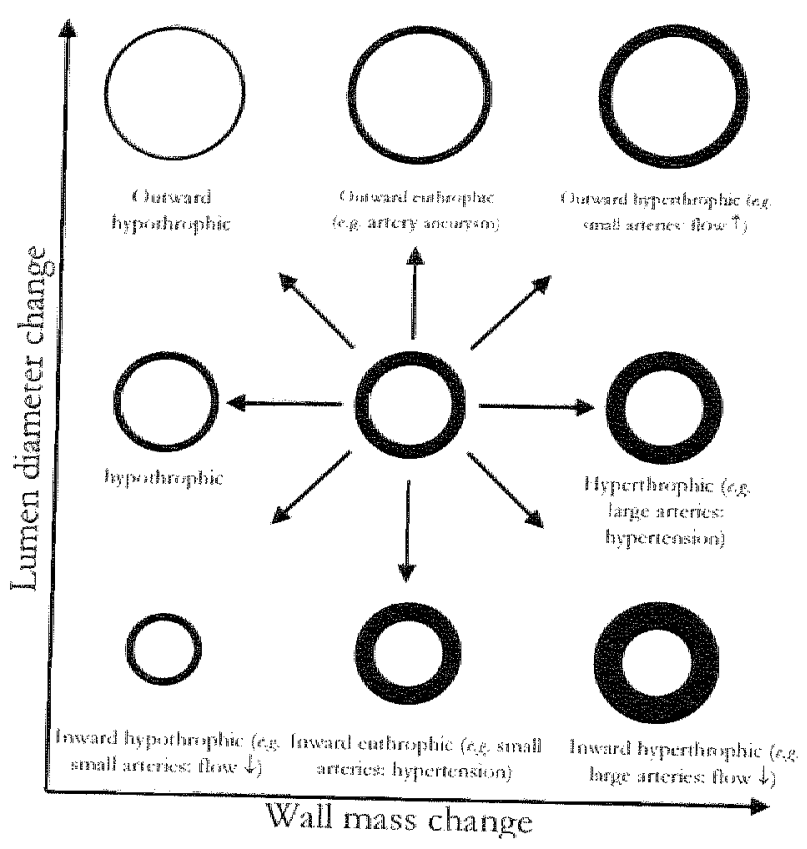

Fignare 1.5. Arterial remodeling werts (adapted fromm) resulting from exposure to chronic alreratons in ransmural pressure and/or blood now. On the vervical axis are lumen diamerce changes denoted as ether inward (decrease in dimerent. ounward (increase in dianeret) or unchanged. Similaty, on the horizonal axis are wall mass changes termed hypotrophic, cutrophic, and hypertrophic as, respecively, a decreased, unchanged, and incteased wall mass.

\section{Physiological Flow-Related Arterial Remodeling}

\section{Pre-and Postnalal dowelopnzent}

The development of the embryonic vasculature depends on the de now formation of new blood ressels (vasculogenesis) and sprouting of new vessels from preexisting vessels (angiogenesis). These processes ane driven by locally released growth factors such as Abroblast growth factor and vascular endorhelial growth factor ${ }^{\text {\% }}$ Further vessel development, va cxpanding of capillary beds and atterialization of feed arteries by enlargement and recrutment of pericytes and smooth muscle cells, becomes thereafter concept was fice act hemodymic forces such as pressute and shear stress. This cmbtyo errew appreciated by Thoma, who observed that arteries in the chicken

Marked birth-related blood fow was high and regressed at low blood flows.

placental blood fow accoun flow changes occur in the perinatal period. In the fetus, Immediately after birth pulmonar for nearly $40^{\circ} \%$ of matemal total cardiac output. ${ }^{222}$ resulting in a 10 fold inctease in pasurestance dramatically falls in the newbota, exchange. ant Closure of the umbilical pulmonary blood flow, allowing pulmonary gas abdominal aorta experiencing a $95^{\circ} \%$ arteries at birth has dramatic consequences for the weeks postpartum in lambs. 31 This was a with a marked reduction in wall mass 2 to 3 cell proliteration with no net DNA accompanied by a reduction of smooth muscle Perfusion of the kidncys and gastroincestinesis) and the occurtence of apoptosis, 
postpartum. ${ }^{45}$ The thoracic aorta supplying these organs incteases $34 \%$ in diameter and undergoes a doubling in wall mass in a period of 2-3 weeks after bith ${ }^{34}$ Changes in vessel diameter and the cellular armangements of the arterial wall constituents affect arterial. viscolasticity. Collagen-to-elastin ratio of the thoracic aorta decreased by $45 \%$ at 3 weeks postpartum in lambs. ${ }^{32}$ Indeed the aortic wall became substantially less elastic at this period afrer birth in neonatal lambs. ${ }^{383}$ These findings indicate that cell turnover plays an important rolle in postnatal arterial growth and development.

These observations confirm the direct sensitivity of arterial growth to altered blood flow, a phenomenon which was shown to be endothelium-dependent. 12 They furthermore indicate that shear stress is the trigger for arterial diamerer adjusments and that blood flow regulates the remodeling of blood vessels in such manner that wall shear stress remains comparable in many arteries and arterioles in newborn and adult animals. $159.57 \%$

\section{Chrowic Exercise}

Durng physical exercise an increase in catciac output and distribution of blood thow to skeletal muscle and the coronary circulation develops. Regular excrcise reduces the incidence of catdiovascular diseases ${ }^{162}$ and lowers risk factors for atherosclerosis. 56 Furthemore, exercise has been demonstrated to improve endothelial function by

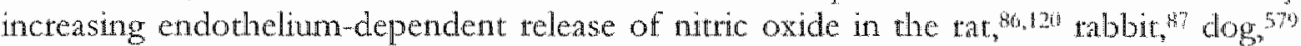
and human ${ }^{205,669}$ and by increasing eNOS expression in dogs. ${ }^{722}$ In patients with coronary artery disease this imptovement of endothelial function during chronic exercise was attributed to increased phosphorylation of eNOS.20 Larget lumen diameters of the common femoral artery were observed in young human adults performing regular exercise compared to untrained healthy controls. ${ }^{127,240,292,589}$ During treadmill exercise in rats, a significant increase in blood flow (reaching up to 50 -fold) to working skeletal muscle develops," structure of arteriolat networks. 314,488

\section{Pregnang}

During mammalian pregnancy uterine blood flow increases dramatically. This tanges from

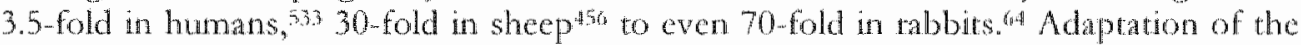
uterine vascular bed to enable sufficicnt pertusion to the growing fetus(es) occurs through augmented dilatation $73,343,58$ outward remodeling of pre-existing vessels and the development of new vessels (angiogenesis). Gh Sex hormones such ns estrogens increasc blood flow to the uterine tissue ${ }^{56}$ via multiple actions such as upregulation of endothelial and neuronal NOS ${ }^{75,337,3 y}$ and vascular endothelial growth factor (VEGP). ${ }^{2 \%}$ VEGF can act as an angtogenic factor 53 and as a vasodiating factor through stimulation of endothelial ft- 1 and $\mathrm{flk}-1 / \mathrm{KDR}$ receptors 212 leading to NOS activition ${ }^{52}$ and subsequent production of NO and prostacyclin.22

Also pregnancy-induced remodeling of the afferent main werine artery (Figure 1.6) contributes substantally to the decrease in uterine resistance. It has been described in a

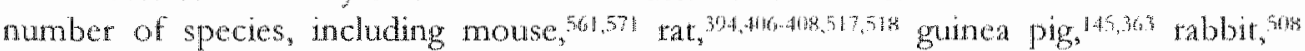
pig. ${ }^{10 \% 4}$ ewe, $11,104,19 \%, 2 \%$ and humans. 59,533 
Immediately after birth, uterine blood low decreases rapidly and the uterine artery regresses toward preptegnant vessel structure representing a plysiological model of reduced blood flow.

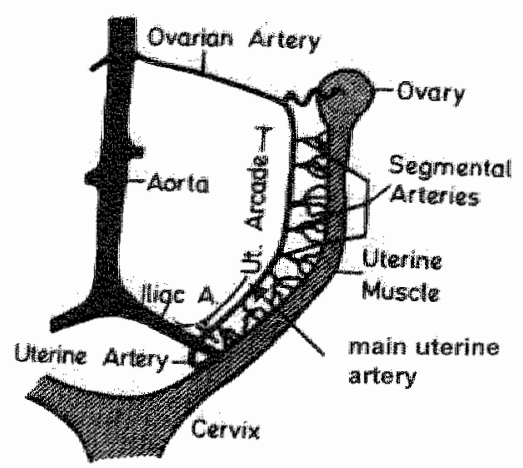

Figure 1.6. Schematic diagram of the left uterine arierial system in rocleses such as rat and mouse. In this thesis the main unerine artery (sec artow) is represented as a segment of the weme arcade, and wot as a scgment of the segmertal arteries (also known as madial arteries), which are biturating from whe man ucrine arkery.

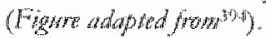

\section{Flow Retated Artenal Remodeltng in Patbologies}

Most vasculat disotders are associated with reduced or inadequate tissue perfusion that ultimately affect arterial remodeling and the severity of the disease. In humans, regions of low shear stress develop inward remodeling and progressive atheroscletosis, $186,338,517,544$ whereas areas of physiological shear stress remain quiescent, and areas of elevated shear stress show outwatd remodeling. 519 The parhobiology of restenosis after percutaneous coronary artery interventions involves atterial remodeling and neointimal hyperplasia. $\left(60,418,41^{03}, 431,432\right.$

Insulin-dependent diabetes mellitus is associated with endothelial dysfunction (reduced vasodilator responses to agonists such as acetylcholine ${ }^{33}$ and reduced flowinduced NO-medinted vasodilatationy. 165,55 Absence of a normal flow response could contribute to decreased michovascular citculation leading to long-tern vascular complications. Decteased MMP activity has been demonstrated in internal mammary arterics of diabetic parients which may contribute to increased collagen deposition and parhological remodeling in diabetes, ${ }^{43}$

Aging is an mpottant risk factor for cardiovascular diseases such as ischemic heart discasc. Athough the mechanisms associated with vascular aging are mulifactorial, one wnderlying pathological cause may be an age-associated endothelial dysfunction 135,603 leading to impaired vasodilator production $25 \%$ and flow-induced dilatation, 181 which predisposes to an increased vascular resistance. "3uthemote, the ability of artertes, and in pariculat the shear stress-sensing ECs, to remodel in response to altered blood flow decreases with advancing age. Reduced flow related arterial remodeling is observed in both large arteries 2301 and resistance arteries. $258.511,554$

Arterial remodeling is often associared with cardiovascular diseases. Striking examples in large elastic conduit arteries include (1) the formation of a neointima that may crolve into a vulnerable plaque ${ }^{31}$ and (2) aneurysm"s which might ultimately butst, $15 \mathrm{O}_{\mathrm{n}}$ the other hand, atretial remodeling can favourably infuence the outcome of catdiowascular disease. For instance, infuences of a growing neointima ot atherosclerotic 
plaque on the patency of the arterial lumen can be limited by timely expansion foutward

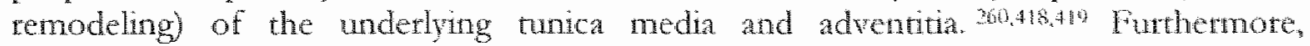
evolution of newly formed or pre-existing collateral arteries into feed arteries can help maintain or restore perfusion of an organ endangeted by a fox-limiting stenosis. ${ }^{7}$

\section{Collateral Artertes}

White mechanical forces such as circumfetertal wall stress and wall sheat stress can

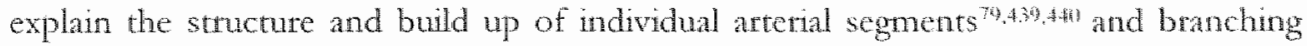
arterial trees, ${ }^{2}+$ there are notable exceptions. Collateral arteries interconnect arterial vessels of the same branching order. They can be formed by angiogenesis and arteriogenesis during pathological or expermentally imposed obstructon of a mior feed

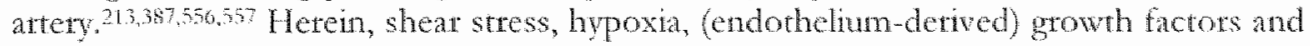
monocyte/macrophages play important roles. Pre-existing collateral atenes are, however, also an integral part of certain rascular beds. In the cerebra circulation of most mammalian species (the gerbil being an excepton) all major food arteries are interconnected at the base of the brain into a structure called the circle of Willis. The uterine arteries that provide the blood supply to the uterus before and during pregnancy, interconnect the ovarian arteries (btanches of the abclominal aorta of of the renal arteries) to the vaginal arteries (branches of the iliac arteries). The circulation to the small intestine is interwined by numerous arterial trees branching off the superior or interior mosenteric arteries. These mesenteric arterial trees are all interconnected at either a proximal level (a. pig) or a distal level close to the gut wall ( 6 gat). In the coronary circulation, the presence of pre-existing collaterals differs extensively benween species ( $6 . g$ dog, many; pig and human, few) which might explain interspecies differences in cardiac consequences of coronary artery occlusion. ${ }^{375}$

In theory, blood pressure is equal at both entry points of a pre-existing collateral artery and there is thus no pressure gradient of net average blood flow over the centre of the collateral vessel. If wall shear stress would be the only determinant of vessel lumen diameter, collateral arteries would be readily lost during development. Their presence in the adult and their occurtence in organs that are characterized by marked fluctuations in blood tlow such as the gastro-intestinal tract and the female uro-genital syetrem, continucs to be a major challenge for current vascular biology. The characteristics of these vessels are of great importance because of their remarkable plasticity in health and discase and because they allow the catrovascular system to cope both funcrionally and stucturally with majot physiological challenges (digestion of large quantities of food; carly growth and development of the offpring) and subchinal pathological situations (obstruction of certain feed artertes).

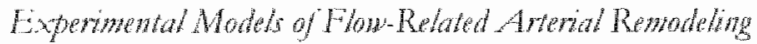

Most expermental animal models to study arterial temodeling in response 10 chironic elevations in blood flow involve creation of an arteriovenous fistula, which can be

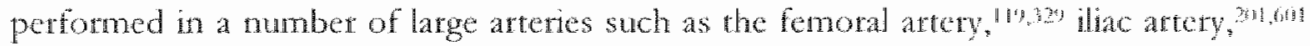

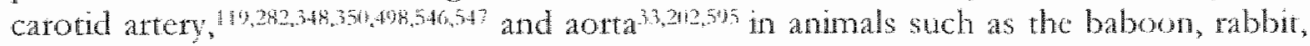
rat and mouse. A major advantage of this method is that supraphysiological blood how levels are obtaned in large arteries. For example, an approximate 30 fold increase in iliac 
artery blood tlow was obtained in monkeyg $g^{60 !}$ and an 11 -fold increase in carotid artery blood flow in the rat. ${ }^{539}$ Another method is the unilateral ligation of the carotid artery, first introduced by Guyton and Hardey, which leads to a reduction of blood flow in the ipsilateral (ligated) artery and a elevation in blood flow in the contralateral right carotid artery. Collaterals interconnecting the facial, cranial and cerebral areas, perfused by both. carotid arteries form the anatomical basis for this shunting. ${ }^{25} \mathrm{~A}$ comparable method was applied in mice by Kumar and Lindner, ${ }^{301}$ resulting in a complete cessation of blood flow in the ligated common carotid artery and a nearly doubling in blood flow in the contralateral catotid artery. A variant of this method is ligation of the extemal carotid artery resulting in an approximately $90 \%$ reduction in carotid artery blood flow. 463 Analogous to the undateral carotid artery ligation, this surgical approach can be applied to side branches of the superior mesenteric artery, resulting in decreased blood flow in ligated mesenteric arteries and shunting of blood flow through collaterals resulting in flow-loaded mesenteric arteries. $71,326,327,434,4,35,54,555$

\section{The Renin-Angiotensin System}

\section{Pbysiologival Funtion and Mediarors}

The renin-angiotensin system (RAS) has traditionally been viewed as an endoctine and circulating system that is important in the short-term homeostasis of cardiovasculat and renal function. It is nowadays well accepted that in addition a local RAS operates in many tissues and blood vessels that regulate local vascular resistance and tissue perfusion. ${ }^{131} \mathrm{~A}$ decrease in blood pressure leads to sympathetic activation and in renal vasoconstiction and hypoperfusion. This stimulates the telease of renin from the renal zonae glomerulosae into the circulation, where it catalyses the transformation of angiotensinogen into the decapeptide angiotensin $\mathbb{I}$. The key enzyme of the RAS is angiotensin I-converting enzyme (ACE), a zinc-containing carboxy-di-peptidase that cleaves the two carboxyterminal aminoacids from angiotensin $I$ to form the potent vasoconstrictor octapeptide angiotensin II (Ang II), ${ }^{139}$ the main biological mediator of the RAS. (Figure 1.7). $\mathrm{ACE}$ is found mainly in the vascular endothelium, where it is bound to the membrane by a hydrophobic membrane-spanning region near the carboxy terminal ${ }^{23.5}$ and is therefore also refetred to as tissue ACE. Protease activity releases ACE into the blood, generating an enzymatically active but truncated plasmatic isoform. Recently, another: ACE-related carboxypeptidase and ACE homologue has been reported (ACE2), which like ACE is membrane-bound and predominanty expressed on the endothelium and restricted in humans to heart, kidney and testis. ${ }^{12}$ It cleaves Ang I into Ang 1-9, which can be further broken down to Ang 1-7, which has opposing effects compared to Ang II, such as diuresis and vasodilatation. 'iat On the other hand, Ang II has numerous, acute and chronic actions, including modulation of vasomotor tone, cell growth, ${ }^{* 1}$ apoptosis, ${ }^{428}$ cell] migtation, ${ }^{3.1}$ extracellular matrix deposition, ${ }^{2+11}$ inflammation, ${ }^{\prime \prime s}$ and stimulation of the production of other vasoconstrictor- and growth factors. ${ }^{82.59}$ 


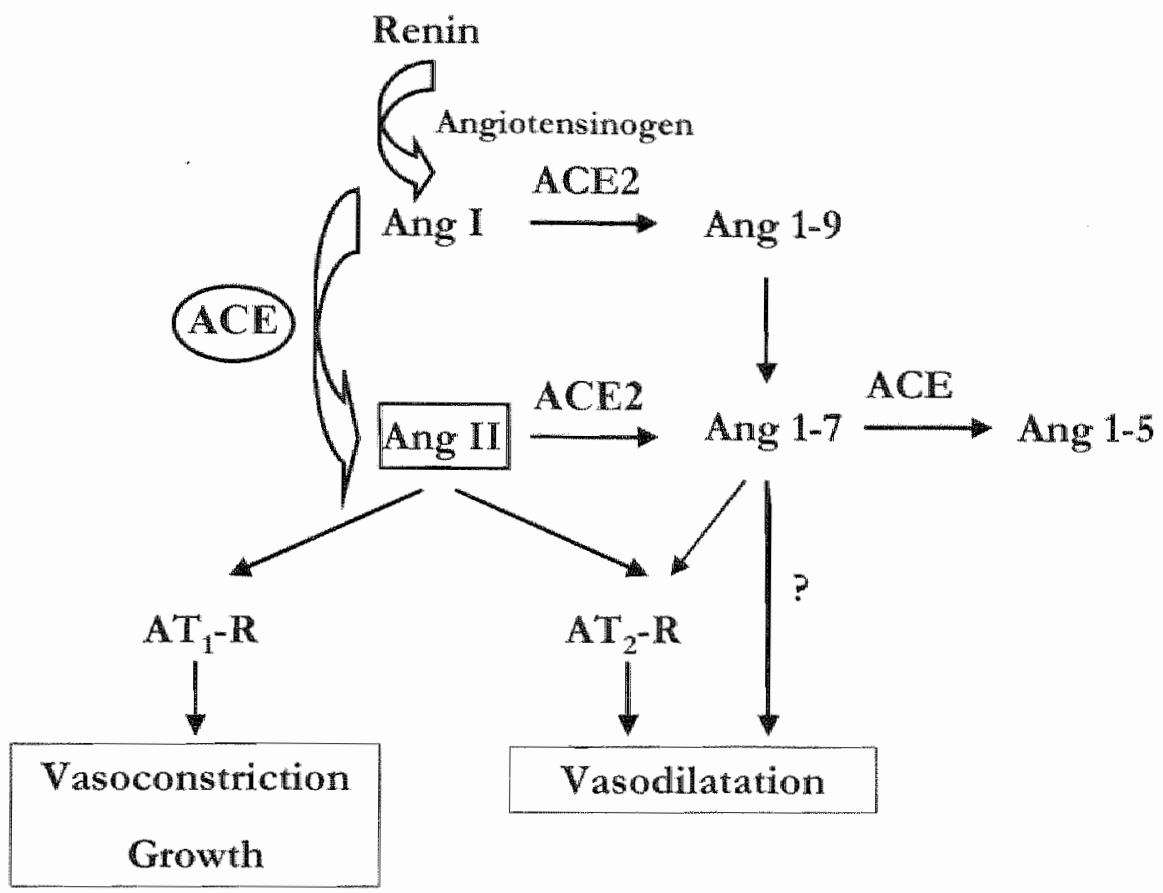

Figure 1.7. Scheme of the renin-angiotensin system. Renin cleaves angiotensinogen to form the decapeptide angiotensin $1(A n g l)$. Ang $I$ is converted by angiotensin l-converting enzyne (ACE) in the lung, tissue and blood vessels to active angiotensin II (Ang ID), which can further be metabolized into smaller peptiades by ACE, ACE2, and other aminopeptidases, such as chymase, cathepsin $G$, or carboxypeptidase. Ang II can bind to its

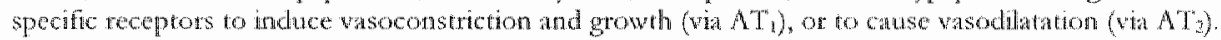

Ang II plays impottant roles in the functional and stuctutal integrity of the atterial wall and in the pathogenesis of cardiovascular discases. medates it effects by binding to specific angiotensin type 1 (Al) and type 2 (AT2) cell membrane receptors. AT receptors have been shown to mediate most of the Ang IIinduced vascular functions. $465 \mathrm{In}$ the vascularure, AT, teceptors ate present at high levels in the media and adventitia, but not on the endothelium, "ust Most adult arteries express predominandy $A T^{*}$ receptors, and the $A T_{2}$ subrype is expressed relarively weakly in large arteries such as the aorta, while a high level of expression is oficn obscrved in resistance arteries. 5.573 "The AT2 receptot. opposes some of the actions mediated by the $A T_{1}$ receptot. $A T_{2}$-deficient mice are hypertensive and have an enhanced ptessot action of Ang $11_{1}^{2}+25$ suggesting that the $A T_{2}$ teceptor plays a role in blood pressure regulation. Ang $I$ has been demonstrated to release $\mathrm{NO}$ wia an endothelium dependent $A \mathrm{Tz}$ receptor

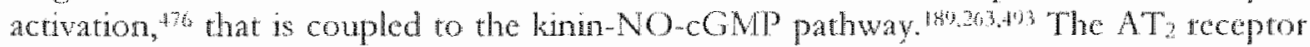

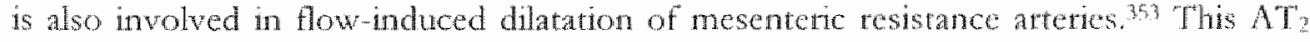
receptor-dependent rasodilatation is mediated by local production of bradykinin in these arteries. ${ }^{29}$. These studies indicate that the $A T_{2}$ receptor mediates vasodiatation in resistance arteries and hereby dectease peripheral resistance and lowering meath arterial pressure. 


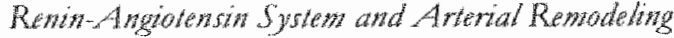

Chronic hypertension is associated with VSMC hypertrophy and increased media deposition of collagen leading to encroachment of the lumen diameter and an increase in vasculat resistance (reviewed in Mulvany). ${ }^{30}$ Pulse pressure, but not the increase in mean arterial pressure is the trigger for the increase in wall thickness, since coarctation of the thoracic aorta in monkeys ${ }^{33}$ and dogs ${ }^{2 n}$ reduced pulse pressure without affecting mean blood pressure, yet still decteased vascular hypertrophy. The importance of the RAS in vasculat remodeling processes came from phamacological intervention studies using $\mathrm{ACE}$ inhibitors (captopril) to prevent the development of hypertension in spontaneously thypertersive rats. ${ }^{163} \mathrm{ACE}$ inhibitors reverse cardiovascular remodeling through reduction of blood pressure, which is an nonecific effect, and through a direct, specific arterial effect (inhibition of VSMC hyperplasia/hypertrophy) that is unrelated to its hypotensive action. Other anthyperrensive drugs, like propanolol, have been shown to lower blood pressure without affecting medial hypertrophy, suggesting that smooth muscle hypertrophy is not simply a response to increased blood pressure, but that other factors,

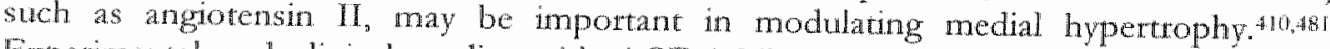
Experimental and clinical studies with $A C E$ inhibitors and $A T_{1}$ receptor antagonists consistently documented beneficial effects of these agents in treating and preventing cardiovasculat diseases such as hypertension, a theroscletosis and testenosis, $36,423,44,44,481$

Although vasoconstriction is the primary function of VSMCs, during development and vasculat temodeling their phenotype changes from a contracrile to a synthetic

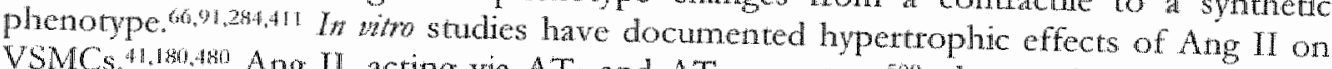

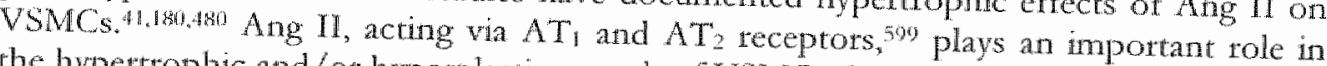
the hypetrophic and/ot hyperplastic growth of VSMCs during increased workload of the arterinl wall occurring during hypertension and during the inflammatory responses involved in atherosclerosis, 202,543 Infusion of Ang II in the rat resulted in a significant DNA synthesis in VSMCs of the aorta and carotid artery, which was suppressed by coinfusion with the $\alpha_{\text {-adrenergic receptor blocker prazosin. }}$. Ad Aditionally, infusion of Ang II increased media thickness in densely innervated mesenteric arteries, whereas no change was observed in the sparsely innervated supenor epigastric artery, indicating that perivasculat nerves are itwolved in the hypertrophe growth stimulus. 31 the hypertophic effecs of Ang II were independent of its blood lowering effects and its increased vasoconstrictor activity. Infusion of non-hypertensive doses of $\mathrm{Ang}$ II in apolipoprotein E-deficiont mice lead to serious abdoninal aotic ancuysms and adventitial remodeling,
that was inhibited by $A T$, blockade and augmented by $A T_{2}$ antagonism.
The prouression of neoing

The progression of neointimal thickening induced by Ang 11 was mediated via AT receptors, in balloon-injured rat carotid arteries 505 A $\Gamma_{2}$ receptors may antagonize the conflicting findings ate observed in $A T_{2}$ receptor knockont mice, showing that $A T$ ?

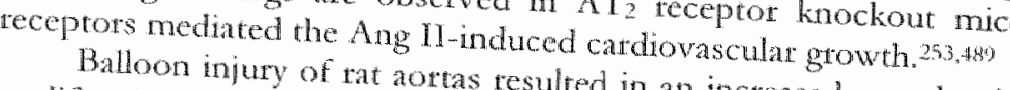
proliferationg neointina. $152,43.44$ The days following balloon injury th $^{\prime}$ Ge carotid artery tevealed an early induction of ACE at 2 are known to stimulate $\mathrm{ACE}$ expression astucocotids and fibroblast growth factor (FGF) 15 arteries with recombinan $\mathrm{CG}^{3}-1$ increased $\mathrm{ACE}$ expression in neointimal $\mathrm{VSMC}$ to the 
same extent as injured, nontransfected arteries. 132 Interestingly greater ACB expression was observed close to the lumen which gradually decreased to the media ${ }^{152}$ suggesting that shear stress and FGF could be involved in the regulation of ACF expression in the neointima. Laminar shoar stress increased ACE activic and mRNA levels of both ACE and bFGF in cultured ECs, VSMCs and fibroblasts." Taken together, these data imply that FGF induces ACE, generating Ang II to stimulate VSMC growth in response to shear stress and arterial injury.

\section{The Kallikrein-Kinin System}

\section{Plysiological Fundon and Mediators}

Kallikreins telease kinins from high and low molecular weight kininogens (Fygure $1.87,80$ Similarly to ACE, plasma and tissue isoforms exist for kallikreins. Human tissue kallikrein cleaves low molecular weight kininogen to release Lys-bradykinin (kallidin), whereas plasma kallikrein teleases bradylinin (BK). ${ }^{34}$ Both peptides are equipotent. Kinins such as BK, Kallidin and Met-Lys-BK act mainly as local homonal oligopeptides by autocrineparacrine mechanisms. Kinins play a role in several (patho)biological processes including renal blood flow regulation, 5 inflammation, ${ }^{3.45}$ and endotoxic shock, in cell culture experiments it was shown that kinins can induce the release of endothelal $\mathrm{NO}$ and prostacyclin, both of which have antiptoliferative and antimigratory effects on VSMCs. 53 Furthemore, kinins can induce the release of interteukins and tumot necrosis factor from macrophages, followed by inducible NOS expression in VSMCs. ${ }^{30,27}$ Kininases degrade the kinins into (in)active fragments. They are found in the blood, ondothelial cells and other tissues. ${ }^{76} \mathrm{ACE}$ (kininase II) removes the C-terminal Phe-Arg from bradykinin and kallidin, linking the renin-angiotensin system with the kallikfein-kinin system. ${ }^{587}$

Based on their pharmacological responses to various BK analogues, two kinin receptors, $B_{1}$ and $B_{2}$, have been characterized. 45 The $B_{2}$ receptors mediate most of the physiological effects of $B K$ and kallidin. $B$ receptors are induced by cottain pathological conditions. 35 Another coupling of the tenin-angiotensin system with the kalikrein kinin system is via AT2mediated vasodilator responses to Ang 1 . Several studies suggest that

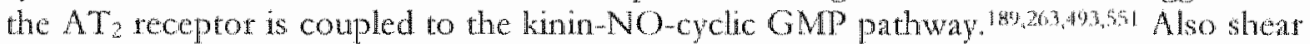
stress can stimulate $A T_{2}$ receptors and vasodilatation through local production of $B K$ in rat mesenteric resistance arteries ${ }^{283}$ and mouse catotid atteric ${ }^{37}, 34$ "This mechanism probably counterbalances the ATinduced vasoconstriction in vits. Tissuc kallikrein localized on ECs likely releases BK in response to sheat stress, but the mechanism by which the activity of tissue kailikrein is regulated remains poony defined. Tsutsumi and colleagues demonstrated that $A T_{2}$ receptor activation causes celluar acidificarion through inhibicion of amblonde-sensitive $\mathrm{Na}^{+} / \mathrm{H}^{+}$exchanger activity that is supposed to activate cissue kallikrein. 5 i 
LMW-Kininogens

HMW-Kininogens

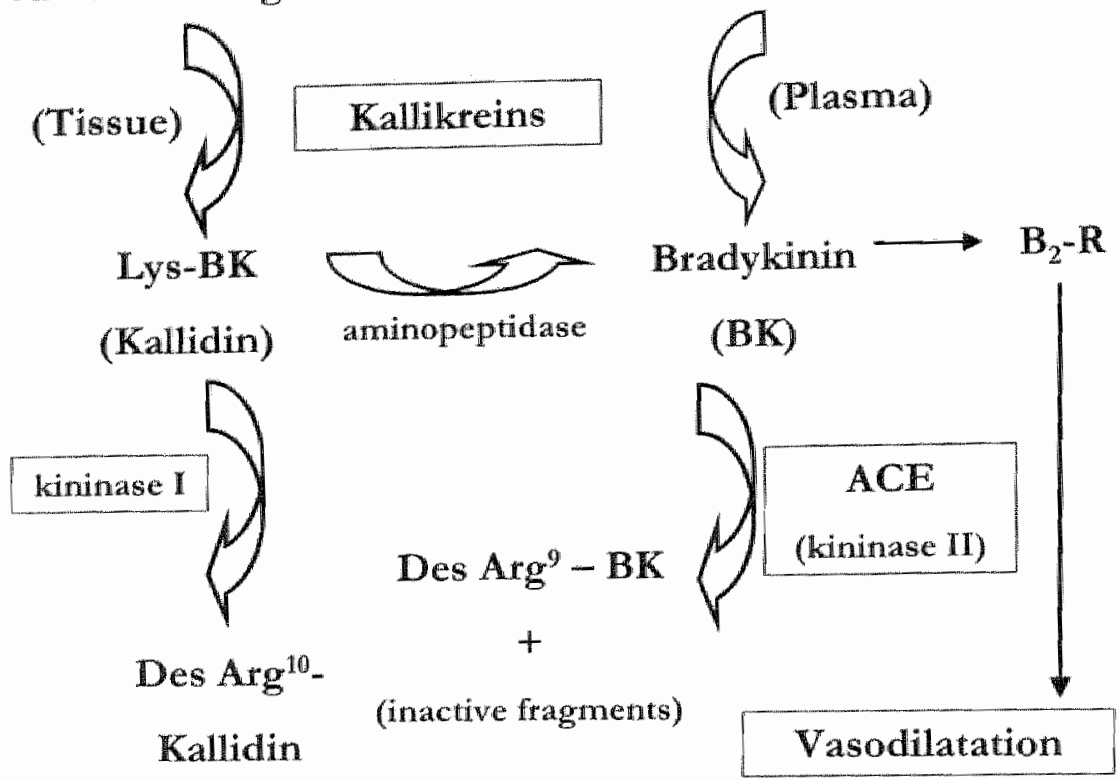

Figure 1.8. Scheme of the kallikein-kinin system. Kinins are released fron big precursor molecules, the kininogens. Low molecular weight (LMW) kininogen is cleaved by usswe kallikrein to release Lys-buadykinin (Kalldin, which can be cleaved into badykinin (BK). High molecular weighe (HMW kinnogen is cleaved by

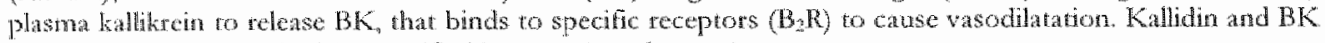
can be further metabolized by specific kininases (see also text).

\section{Kallikerein-Kinin System and Anterial Remodeling}

Potentation of the endogenous kinins via ACE inhibition has antihypertensive actions. This was demonstrated in a number of different rat models of hypertension after administration of ACE inhibitors such as cnalapriat, tamipril and captopril. Blockade of the $B_{2}$ rcceptors has been shown to attenuate the blood-lowering effect of $A C E$ inhibitors in these studies. ${ }^{12,35,2,105}$ In rabbits on a long-term atherogenic diet, ACE inhibitor treatment prorected against the progressive endothelial dysfunction observed in atherosclerosis. $34,4,2$

The protective cffects do not firmly discriminate between reduced Ang II production, increased levels of BK or increased $N O$ and prostacyclin production by $A C E$ inhibition. In apolipoprotein E-deficient mice the antatherogenic effects of ACE inhibition wete independent of its blood lowering and BK relating effects, ${ }^{285}$ suggesting what the disadvantageous effects are mediated by Ang II. Kinins have been documented to mediate the antiproliferative actions of ACE inhibition after rat carotid artery endothelial denudation, ${ }^{148}$ an effect which was NO-mediated and $B_{2}$ teceptor-dependent. ${ }^{19}$ Other studics have shown absence of antiproliferative actions of ACE inhibition on VSMCs, whereas $A T_{1}$-blockade did. Prescott and colleagues demonstrated that in the carotid artery 
balloon injury model, $A C E$ inhibition reduced neointimat formation by inhibiting $5 \mathrm{MC}$ migration alone without affecting SMC proliferation. ${ }^{43}$ "They found a more prononuced reduction in lesion size after $A \mathrm{~T}_{1}$ antagonism, however, when both $\mathrm{SMC}$ migration snd. proliferation wete inhibited. Thus, combined ACE inhibition and $A T_{1}$-blockade reduced neointima formation, which further demonstates the importance of Ang II in VSMC migtation and proliferation after atterial injury, 4.38

In rabbits, the AT, teceptot, and not the AT2 receptor, was implicated in neointimal hyperplasia after carotid artery balloon injury. 265.5 In anothet model of neointima fomation, ACE inhibition markedly reduced the neotntimal formation after blood flow cessation in mouse carotid arteries. ${ }^{130}$ Furthemore, gene transfer of human tissue kallikrein, the kinin-forming enzyme in the endothelium, inhibited neointirna formation in the same model, which was mediated via $B_{2}$ receptor signaling. "These results demonstrate a role for kinins in suppressing VSMC growth and migration, but again do not discrimnate evidently between the beneficial actions of kinins and the proliferating actions of $\mathrm{Ang}$ II in neointima formation. Perhaps receptor heterodimerization of $A T_{1}$ and $B_{2}$ receptors occurs, just as has been shown in preeclampsia."

\section{Relations Between Acute and Chronic Arterial Responses to Mechanical Stimuli}

Although mechanical stimuli such as CWS and WSS can modulate vasomotor tone and arterial structure, the relation between the acute functional and chronic structural responses remains unclear. Several possibilities have been considered: (1) the functional response (.$g$. more or less constriction) might give rise to the functional response (e.g. more or less growth and reorganization of the extracellular matrix), ${ }^{16,17}$ (2) the same mediators might participate such as the vasodilator and growth-inhibitory substances NO, prostacyclin and bradykinin or the vasoconstrictor agonist and growth factor angiotensin II and (3) distinct processes might be involved. In the case of the second hypothesis, conditions that chatacterize candiovascular disease such as endothelial dystunction 1.35 , and activation of the renin-angiotensin system ${ }^{179}$ would entail alterations of both the acute and the chronic compensatory responses to mechanical stimuli. In the case of the third hypothesis, the chronic structural tesponses would come into play as a secondary back-up protective mechanism when the acute functional responses would fail to nomalize the mechanical forces.

Below, components of these wotking hypotheses are combined with respect ro nowinduced vasodilatarion and flow-induced outward arteral remodeling (Figure 1.9). It is proposed that an acute moderate rise in blood flow, for instance during physical activity, will lead to an inctease in WSS, causing telease of endothelium-cletwed vasodiatory compounds that dilate the vessel. This fow-induced vasodilatation restores the WSS value toward nomal (upper part Figure 1.9). However, when the rise in blood flow is large and persistent, flow-induced dilatation can not fully restore WSS. Structural rearrangement of pre-existing wall material leads to a widet diameter and hence a thinner wall. The formet will rend normalize WSS, while as a result of the latret CWS will be increased and hypertrophy will be srimulated (lower part, Figure 1.9). 


\section{Small BF increases: Large BF increases:}

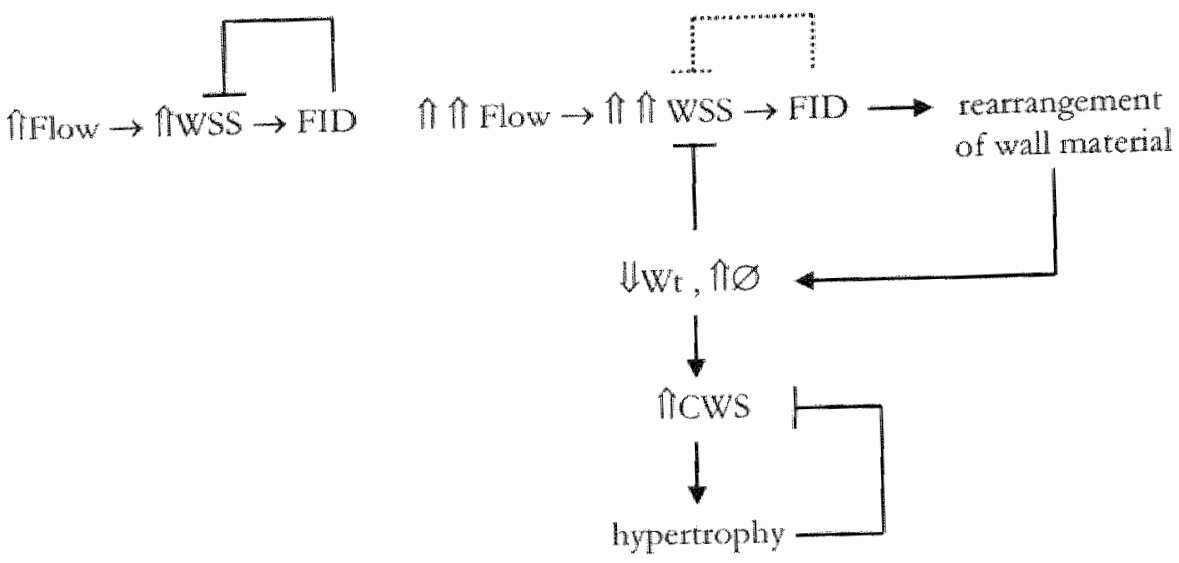

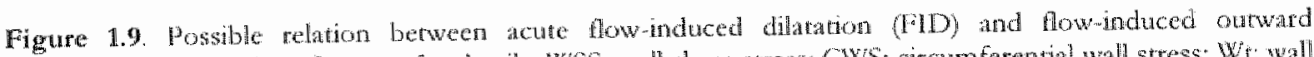

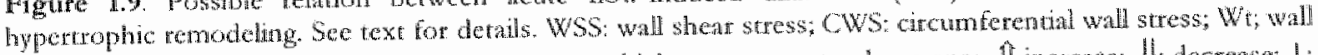

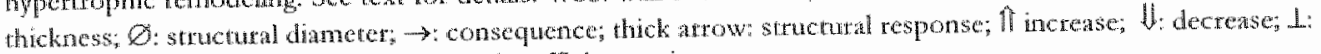
negreve feedback regulation; dorted line insufficientaction.

\section{Novel Experimental Animal Models}

Traditionaly surgical and phamacological intervention studies have been applied as a model for (cardio) vascular remodeling. The use of dugs as a rool for chronic inhibition of an enzyme or a recptor system is limited to (1) the antagonists specificity and affinty for the enzyme, (2) the metabolic degmadacion rate, (3) transport to the site of action. Nowadays, a growing number of trangenic mouse models are being used for disease resench and drug development. Transgenic animals are animals in which foreign genes have been experinentally introduced or endogenous DWA deleted from. Transgenic mice can be defined as a gain of function or loss of function. Gain of function is associated with an overexpression of randomly integrated trasgens fragments foreign DNA) into fertilized eggs of mice. Targeting the deletion or tautation of a specific gene using cmbryonic stem cell technology creates loss of function ot knock-out models. Both of these two groups of tranggenic mice offer a myrad of models to study individual genes and gene products in the process of, for instance, development and disease pathogenesis. Many ansgenic mouse models mimic the phenotypes of the human diseases. Transgenic models have the main advantage that the effect of one specific gene or gene product can be studied, although many genes have pleiotropic roles. This problem can be overcome by cell rype-selective and conditional transgenic technology. The potential disadvantages of ansgenic anmals are outweighed by the unique opportunity for developing and testing 
different therapeutic strategies betote they ate introduced in the clinic. Many transgenic mice have been used in the field of vascular biology to study the mediators inwolved in neointima fomation, smooth muscle cell migration, matrix degradation, atherosclerotic placke, etcetera. However, few transgenic mice models were used to study flow-related artenal remodeling. Mice lacking one cytosketal protein (such as desmin or vimenrin) show blunted acute flow-induced diatation of mesenteric tesistance arteries, ${ }^{220,327}$ Despite this acure arteral dysfunction, longtem arterial stuctural adaptations to blood flow wete unaltered in these knock-out mice compared to will-type mice. ${ }^{32.479}$ In this thesis two strains of genetically-altered mice wete used: tissue kallikrein-deficient (TK $/ 9$ mice and tissue angiotensin I-converting enzyme-deficient ( $t-A C E$ ) mice. The lack of rissue kallikrein has been shown to abolish the kinin-forming capacity in most dissues and to induce cardiac and vascular abnomalities, without changing blood pressure. ${ }^{3,3}$ Flowinduced vasodilatation in response to shear stress of carotid artetics was blunted in these

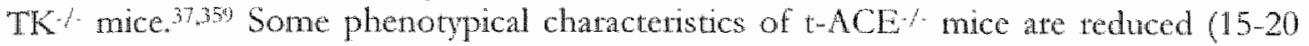
mmHg less than nomal) mean arterial bload pressute, teduced male fertility, and renal vascular thickening. "1n the future, many mote genetically engineered animal models will be valuable for gaining important insights into the molecular mechanisms underying development, disease pathogenesis, prevention and treatment:

\section{Aim and Outline of the Thesis}

The present thesis aims to determine the roles of vasoactive peptides/growth factors in flow-induced atterial dilatation and flow-related arterial remodeling. Two interdigitating systems, the renin-angiotensin system and the kallikrein kinin system, which are regarded as opposing systems in blood pressure control, were evaluated to elucidate the contribution of these systems and their main players in acute and chtonic responses of both small and large arteries to altered blood flow (Figure 1.10),

Two models of flow-related arterial remodeling were studied. The first model is murine pregnancy, which like all mammalian species, reptesents a physiological model of increased utetine blood flow to the growing hetus(es), resulting in considerable arteriogenesis of the afferent uterine wasculat bed. The second model is unilateral carotid artery ligation of the mouse, which tepresents an experimentally-imposed model of a chronic cessation of blood flow in the ligated carotid artery and a doubling of blood low in the contralateral carotid artery. In order to investigate arterial function (wasomotor responses to mechanical stimuli, vasoconstrictors and vasodilators, and remodeling (diameter and wall mass changes), reactivity studies and histological techniques were pertormed in isolated, pressurized uterine and catotid arteries.

Tissuc kallikrein-deficient mice were studied in Chapters 2 to 4 to analyo the effect of an absence of membrane-bound kallikein (and reduced bradykinin synthesis) on acute vasomotor responses and chromic structural responses of carotid arteries (Chapter 2 and 3) and uterine arteries (Chapter 4) to flow. The reninangiotensin system, and especially tissue angiotensin-l-converting enzyme, was the focus of Chapters 6 and 7 . Arterial structure and elasticity of large conduit arteries and peripheral antreries were analyzed in tissue angiotensin. Iwconverting enzyme deficient mice (chapter 6). Agonist- and flowinduced dilatation were investigated in isolated werine artcrics of nompregnant, late 
pregnant and 7-days postparum mice (Chapter 5). The effects of chronic exposure to altered blood flow, applying the two models of flow-related arterial remodeling, wire detemined in these mice (Chapter 7). The consequences of aging on acute and chrotic uterine attery responses to flow were investigated in one-yeat old wild-rype nonpregnat and late pregnant mice in comparison to young (3-months) mice (Chapter 8). A review of flow-related arterial remodeling and to telationship to flow-induced vasodilatation is presented in Chapter 9. Finally, a general discussion of this thesis is ontined in Chapter 10.

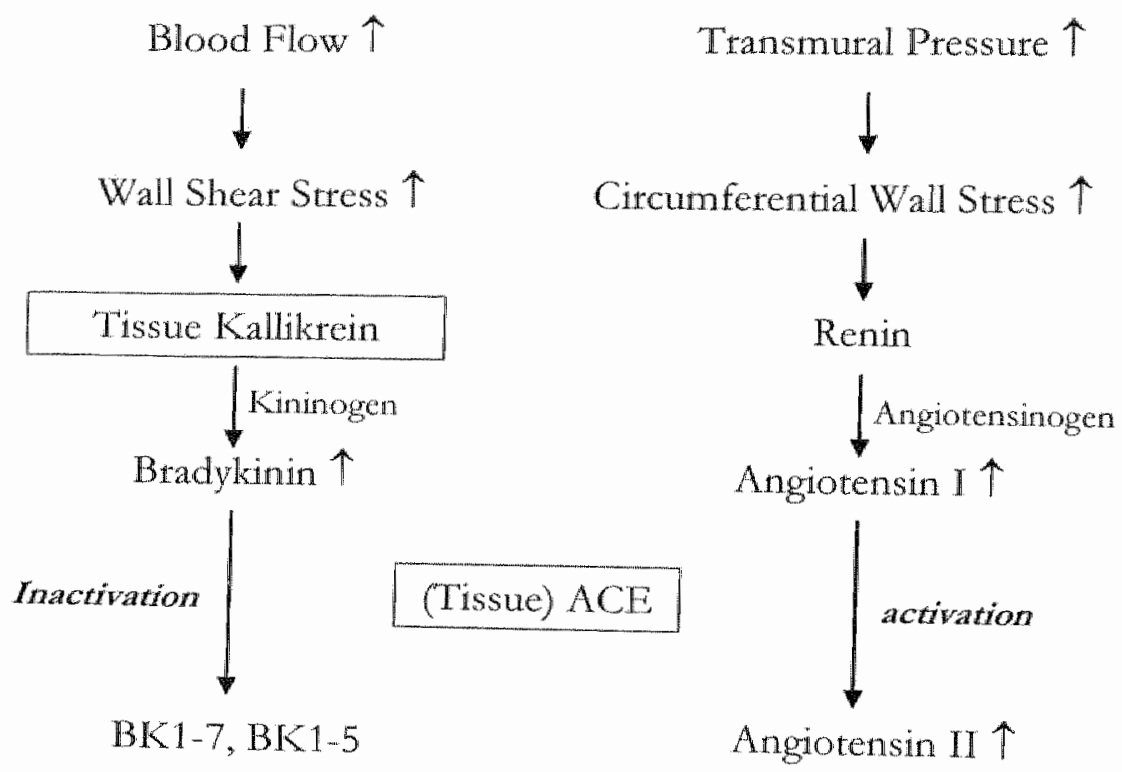

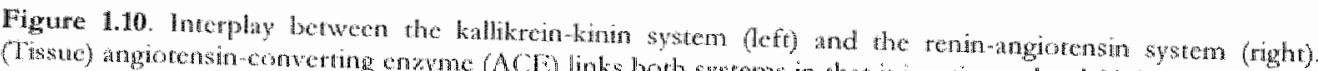

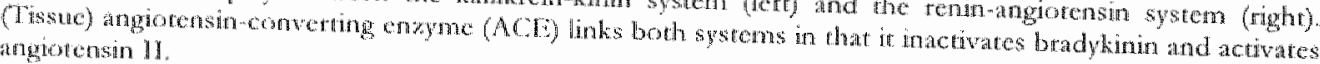


Chapter 2

\section{Flow-Dependent Dilatation Mediated by Endogenous Kinins Requires Angiotensin $\mathrm{AT}_{2}$ Receptors}

Sonia Bergaya, Rob H.P. Hilgers², Pietre Meneton", You Dong, May Bloch-Faure, Tadashi Inagami", François Alhenc-Gelas", Bernard I. Lévy!, Chantal M. Boulanger!.

Instiut National de la Santé er de la Recherche Médicale (INSERM), Unit 541, Hôpital Lariboisic̀re, Patis, France,

2Cardiovascular Research Institute Maastriche (CARIM), Universiteit Maastricht, the Netherlands,

3NSERM, Unit 367 , Paris, France,

4 Vanderbilt University School of Medicine, Nashville, Texas, USA.

Gir Res. In Press 


\section{Abstract}

Objective. The vascular kallikrein-kinin system contributes to flow-inducod dilatation by activating bradykinin $\mathrm{B}_{2}$ receptors coupled to endothelial nitric oxide (NO) release. Because the bradykinin/NO pathway may mediate some of the effects of angiotensin II $\mathrm{AT}_{2}$ receptors, we examined the possible contribution of $\mathrm{AT}_{2}$ teceptors to the kinin-dependent response to flow.

Methods and Results. Changes in outer diameter following increases in flow rafe were evaluated in perfused arteries from wild-type $\left(\mathrm{TK}^{+/+}\right)$and tissue kallikrein deficie $\mathrm{l}_{\mathrm{i}} \mathrm{t}$ (TK\%) mice in which the presence of $\mathrm{A}_{2} \mathrm{~T}_{2}$ receptor expression was vertified. Saralasin, a non-selective angiotensin II receptor antagonist, significantly impaired flow-induced dilatation in TK+ ${ }^{+/}$, whereas it had no effect in TK mice. In both groups, blockade of $A T_{1}$ receptors with losartan or candesartan did not affect the response to flow. Inhibition of $\mathrm{AT}$ 2 receptors with PD123,319 significantly reduced flow-induced dilatation in TK mice. Combining PD123,319 with the bradykinin $\mathrm{B}_{2}$ receptor antagonist HOE-140 hat no additional effect to $A T_{2}$ receptor blockade alone in $T K^{+1+}$ arteries. Flow-dependent dilatation was also impaired in $A T_{2}$ receptor deficient mice $\left(A T_{2}^{-1}\right)$ when compared $t$, $\mathrm{AT}_{2}+1+$ litremates. Furthermore, HOE 140 significantly reduced the response to flow it: the $\mathrm{AT}_{2}{ }^{* /+}$, but not in $\mathrm{AT}_{2}^{*} \%$ mice.

Conclusion. This study demonstrates that functional $A T_{2}$ receptors play a key role in flow-induced dilatation mediated by the vascular kalliktein-kinin system. 


\section{Introduction}

Kallikreins are a distinct group of setine proteases, which are capable of generating vasoactive kinins from kininogen in vitro. Vascular tssue contain kininogen and kallikrein mRNA suggesting the existence of an endogenous kallikrein-kinin system (KKS). Tissue KKS is present in both endothelial and smooth muscle cells of vartous vascular territoties and is different from the circulating plasma KKS $39,41,34,596$ Indeed, tissue kallikrein (TK) is encoded by the kallikrein ke/k1 gene, and experimental evidence suggests that endogenously formed kinins could participate in the regulation of vascular tone. ${ }^{176,365,366.469,587}$ Recently we reported that tissue kallikrein plays an essential role in kinin synthesis within the vascular wall of mutine carotid arteries. ${ }^{37}$ In addition, this tissue KKS contributes to the flow-induced dilatation though activation of bradykinin $B_{2}$ receptors leading to NO release in these arteries. "Flow-induced dilatation enables arteries to increase their diameter in response to elevations in blood flow following the release of endothelial-derived factors such as NO or prostacyclin to ensure appropriate organ perfusion. ${ }^{1612,269,427,461}$ Flow-induced dilatation is a complex response involving not only local mediators (such as kinins), but also mechanical activation of endothelial cells. Other peptide systems such as angiotensin II (Ang II) can also contribute to flow-induced dilatation by activating $A T_{2}$ receptors, as demonstrated in resistance arteries. ${ }^{353}$

Interestingly, several studies suggested a possible link between Ang II receptors and

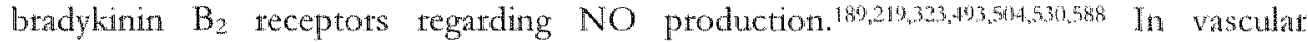
endothelial cells, the production of NO by Ang II is caused by activation of the angiotensin type $2\left(\mathrm{AT}_{2}\right)$ receptor. ${ }^{588}$ In addition, $A T_{2}$ teceptor stimulation by exogenous Ang II leads to an increase in cyclic GMP, through a mechanism involving bradykinin $\mathrm{B}_{2}$ receptors and NO release. ${ }^{189,219,283,493,504,530,56,3}$ Furthermore, overexpression of $\mathrm{AT}_{2}$ receptors increases bradykinin production presumably by activating kininogenase(s). ${ }^{5.51}$ However, there is no information regarding a possible telation between $A T_{2}$ and bradykinin $\mathrm{B}_{2}$ receptor pathways during flow-induced dilatation. Therefore, the purpose of the present study was to determine whether $A T_{2}$ receptors contribute to flow-induced dilatation mediated by endogenously formed kinins using isolated carotid arteries from $\mathrm{TK}^{+1+}$ and $\mathrm{TK} /$ mice.

\section{Methods}

\section{Animals}

Twelve-week old male littermate wild-type (TK ${ }^{+/ 4}$ ) and tissue kallikwein knockour (TK/) mice were used as previously described. $3,35 \%$ Twelve-week old male $A T_{2}$ receptor deficicnt mice $\left(\mathrm{AT}_{2} / \mathrm{)}\right.$ ) and their age-matched $\mathrm{AT}_{2}^{+1++}$ littermates were generated as described ${ }^{252.494}$ They were backcrossed ten times in the C57BI/6J genetic background.

\section{RNA Extraction and RT-PCR Analysis}

For each group of mice, carotid arteries from ten mice were pooled and total RNA was extracted according to the Trizol reagent protocol (Life Technologies, Cergy Pontoise, France). The quality of the RNA preparation was confirmed by ethidium bromide staining. 
Single-strand cDNA synthesis was carried out in $20 \mu$ of reaction buffer, consisting of first-strand buffer $5 X$ (GibcoBRI, Cergy Pontoise, France), RNase inhibitor (40 UI/HL), di-nucleotideriphosphate (25 mmol/L), DTT (100 mmol/1, Amersham, Orsay, France), and reverse MMLV (200 UI/ HL). The reverse transciptase reaction was perfomed by incubating the reaction mixture for $90 \mathrm{~min}$ at $37^{\circ} \mathrm{C}$ followed by $10 \mathrm{~min}$ at $65^{\circ} \mathrm{C}$. using a $3^{\prime}$ oligo-(5'-GGTTTCCAAGGAAGGGTATGAG). The polymerase chain reaction (PCR) included three steps: denaturation $\left(94^{\circ} \mathrm{C}, 45 \mathrm{~s}\right)$, annealing $\left(65^{\circ} \mathrm{C}, 45 \mathrm{~s}\right)$, and extension $\left(72^{\circ} \mathrm{C}, 105 \mathrm{~s}\right)$, for 35 cycles using the above mentioned $3^{\circ}$-oligo and the $5^{\prime}$-oligo (5'-CATGCTTTTGTTCTGGGCTTCGTC). These primers were taken from the unique exon from position 2,176 to 2,749 of the angiotensin $A T_{2}$ teceptor gene, localized in chromosome $X$, thus producing a $0.573 \mathrm{~kb}$ fragment of the $A T_{2}$ receptor $C D N A 388$ The CDNA was amplifed using $5 \mathrm{UI} / \mu \mathrm{L}$ of Tag DNA polymerase (Life Technologies, Cergy Pontoise, France) and $20 \mu \mathrm{mol} / \mathrm{L}$ of each set of primers in $50 \mu \mathrm{buffer} 10 \mathrm{X}(22 \mathrm{mmol} / \mathrm{L}$. Tris-HCl; $\mathrm{pH} 8.4,55 \mathrm{mmol} / \mathrm{L} \quad \mathrm{KCl}, 1.65 \mathrm{mmol} / \mathrm{L} \quad \mathrm{MgCl}_{2}, 25 \mathrm{mmol} / \mathrm{L} \mathrm{di}-$ mucleotideriphosphate, and $10 \mu \mathrm{L}$ of loading dye $(0.02 \% \mathrm{red}$ cresol and $60 \%$ sucrose). The PCR products were scquenced and corresponded to the mouse $A T_{2}$ receptor sequence.

In addition, expression of GAPDH was evaluated in parallel to that of the AT2 receptor. Briefly, total RNA was reverse transcribed as mentioned above using the 3'oligo-(5'CATGTAGGCCATGAGGTCCACCAC). Then, the RT product was amplified by 35 cycles as described abowe, using the 3'-primer and the 5'-oligo $\left(5^{\prime}\right.$ TGAAGGTCGGTGTGAACGGATTTGGC). The migration of both PCR products was then followed by elecrophoresis on a $2 \%$ agarose gel containing ethidium bromide.

\section{In Viwo Measurwent of Anterial Dianneter}

Carotid atteries were carefully exposed and quickly excised. The procedure was in accordance wirh the European Community guidelines on the care and use of laboratory animals (Ministète de l'Agriculture, Ftance, authorization 07430). Carotid arteries were cannulated at both ends and then perfused continuously in witro in a videomonitored arteriograpl setup (1.iving Systems lnstrumentation, Burlington, VT, USA), whete fow and pressure can be modified independendy, as previously described. ${ }^{3}$ Briefly, arteries were bathed in a physiological salt solution wamed $\left(37^{\circ} \mathrm{C}\right)$ and gassed $\left(95 \% \mathrm{O}_{2} / 5 \% \mathrm{CO}\right)$ throughout the experiments. Intrahminal pressure was monitored by a servoperfusion system. The onter diameter as well as proximal and distal intralummal pressures were contmuously recorded. The integrity of the endothelim was assessed by extraluminal

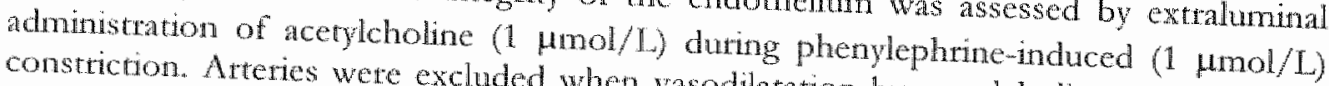
constriction. Arteries wete exchded when vasodilatation by acetylcholine was $<60 \%$ of
that induced by Na-nitroprusside $(0.1 \mathrm{mmol} / \mathrm{L})$. At the end of each experiment, passive
diameter was obtained following incubation of the anter physiological salt soluron and $\mathrm{Na}$-nitroprusside of the artery $(40 \mathrm{~min})$ with $\mathrm{Ca}^{2}$-free muscle tone. Phenylephine and all aptusside $(0.1 \mathrm{mmol} / \mathrm{L})$, in the absence of smooth extrailuminally. 


\section{In Tho Protociols}

All experiments evaluating the response to increases in intaluminal fow were performed in the presence of phenylephrine ( $1 \mu \mathrm{mol} / \mathrm{L})$. When atable constriction was reached, intrahminal flow was applied in a stepwise manter (from 10 to $800 \mu \mathrm{L} / \mathrm{min}$ ). Fach low was applied for 3.9 min until a stable diameter was reached, and then augmented to the next level. Experiments were performed in the presence of either, saralasin (a nonspecific $A T_{1}$ and $A T_{2}$ blocker; $1 \mu \mathrm{mol} / \mathrm{L}$ ), losartan (an $A T_{1}$ blocker; $0.1 \mu \mathrm{mol} / \mathrm{L}$ ), candesartan (an AT, blocker; $10 \mathrm{nmol} / \mathrm{L}$ ), PD123,319 (an AT2 blocker; 1 Hmol/L), or HOE-140 (a bradykinin $B_{2}$ receptor blocker; $1 \mu \mathrm{mol} / \mathrm{L}$ ). Tissues were preincubated for 40 min with each blocker.

Some experiments were perfomed on isolated mesenteric arteries, which wete mounted as described above. Mesenteric artery basal dianeters in $A T_{2}^{+/ t}$ and $A T_{2}^{*}$ mice were $177 \pm 12 \mu \mathrm{m}(\mathrm{n}=7)$ and $181 \pm 20 \mu \mathrm{m}(\mathrm{n}=6)$, respectively. The arteries were perfused (40 $\mu 1 / \mathrm{min}$ ) at an intraluminal pressure of $80 \mathrm{mmHg}$, and diatation to cumulative concentations of extraluminally administered bradykinin $(0.1 \mathrm{mmol} / \mathrm{L}-10 \mu \mathrm{mol} / \mathrm{L})$ was recorded during preconstriction with the thromboxane analogue $U 46619(1-10 \mathrm{mmol} / \mathrm{L})$.

\section{Drwgs}

The compounds used for in vitro studies included acetylcholine chloride, bradylkinin diacetate salt, L-phenylephrine hydrochloride, U46619 (Calbiochem, France), PD123,319, saralasin, and losartan (from Sigma, St-Quentin-Fallavier, France). HOE 140 was kindly provided by Drs. H.). Lang and B.A. Schökens (Hoechst-Marion-Roussel, Erankfurt, Germany). Candesattan was kindly provided by ASTRA-ZENECA.

\section{Data Analysis and Statistico}

Data are given as changes in diametet" (mictons) from the atterial diameter obtained during constriction wirh phenylephrine. Results are expressed as moans $56 \mathrm{M}$ of $n$ experiments; $n$ represents the number of animals used for each experimental protocol. $\mathrm{pD}_{2}$ values represent the negative logarithm of the concentration of agonist causing $50 \%$ of its maximal response. Statistical evaluation was performed by using ANOVA for factorial or repeated measurements, followed by Scheffe $t$-test. ${ }^{575}$ Vahes of $P<0.05$ were considered to be statistically significant. 


\section{Results}

RT-PCR revealed the expression of $A^{\prime} T_{2}$ receptors in carond arteries of $T K K^{+/ 4}$ and $T K^{*}$ mice (Frgure 2.1). In perfused carotid arteries of $T K+$ and $T K$ mice, administration of phenylephrine, acetylcholine or Na-nitroprusside caused similar changes in outer diameter for each set of experiments (Table 2.1).

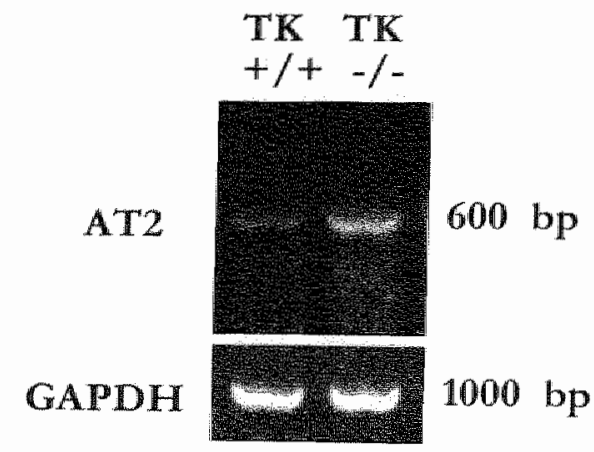

Figure 2.1. Expression of the angiotensin II $A \mathrm{C}_{2}$ receptor gene in cartorid arteries of $\mathrm{TK}$ and " $\mathrm{TK}$ mace This typical Rl.PCR experiment is a representative of total of two different experiments obaned on different pools of 10 carotid arteries. Expression of $\mathrm{CAPDH}$ was: obserwed in the sume samples in parallel experiments.

Step increases in intraluminal flow augmented the carotid artery diameter in both TK+1+ and " $T K$ " mice (Figure 2.2). At high flow rates flow-induced dilatation (FID) was significantly lower in arteries from $T K^{*}$ mice compared to $T K^{+/ 4}$ as previously
observed. ${ }^{37}$

Table 2.1. Changes in outer diamete of carotd atteries from "TK" and "TK mice, in response ro phenylephrine, acetylcholine, and $\mathrm{N}_{\mathrm{a}}$-nitroperside in the absence and presence of antagonsts

\begin{tabular}{|c|c|c|c|c|c|c|c|}
\hline Genotype & Agonist & Control & Losartan & $\begin{array}{c}\text { Losatran + } \\
\text { PD123,319 }\end{array}$ & Satralasin & PD123,319 & $\begin{array}{c}\text { PD123,319 + } \\
\text { HOE } 140\end{array}$ \\
\hline \multirow{3}{*}{$T \mathrm{~K} / \mathrm{s}$} & Phe & $-58 \pm 5$ & $-56 \pm 11$ & -7418 & $-65+14$ & $-67 \pm 14$ & $-70 \pm 1.4$ \\
\hline & $\mathrm{ACh}$ & $94 \pm 5$ & $91 \pm 4$ & $93+6$ & $100+2$ & $99 \pm 3$ & $99+3$ \\
\hline & $S N$ & $83 \pm 12$ & $80 \pm 16$ & $93 \pm 11$ & $01 \pm 2$ & $77 \pm 15$ & $83 \pm 15$ \\
\hline \multirow{3}{*}{$\mathrm{TK}$} & Phe & $-58 \pm 8$ & $-71 \pm 7$ & $-72+9$ & $-63 \pm 12$ & $-68 \pm 9$ & $\mathrm{ND}$ \\
\hline & $\mathrm{ACh}$ & $94+4$ & $85+5$ & $84 \pm 4$ & $101 \pm 1$ & $95 \pm 4$ & $\mathrm{ND}$ \\
\hline & SNP & $72+10$ & $70+8$ & $78 \pm 9$ & $74 \pm 14$ & $92 \div 9$ & $\mathrm{ND}$ \\
\hline
\end{tabular}


In TK+t mice, EID (between 200 and $800 \mu$ L/min) was significanty impaired by the nonspecific angiotensin teceptot antagonist saralasin (Figure $2,2, \mathrm{lf}$ ). Conversely, saralasin did not significandy modify IJD of TK/ carotid arteries (Figure 22 , mhin). We next investigated the effect of preferental $A T_{1}$ and $A T_{2}$ receptor antagonists on $\mathrm{FDD}$. The AT receptor blocker losartan did not affect FID in both $\mathrm{TK} /$ and $\mathrm{TK} /$ arteries (Figure 2.3). Similarly, candesartan had no effect on FID (data not shown).

\section{$\mathrm{TKK}^{+/+}$}

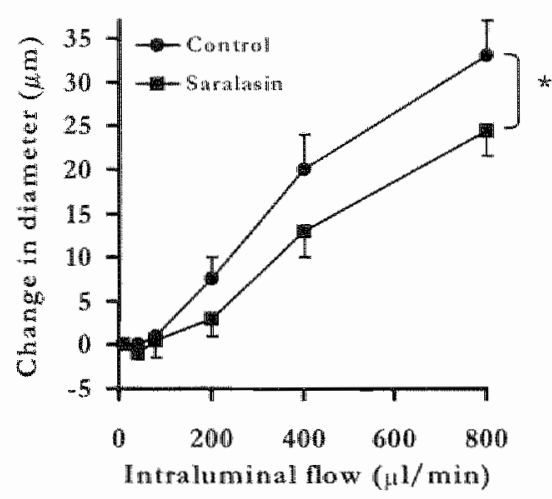

$\mathbf{T K}^{-1-}$

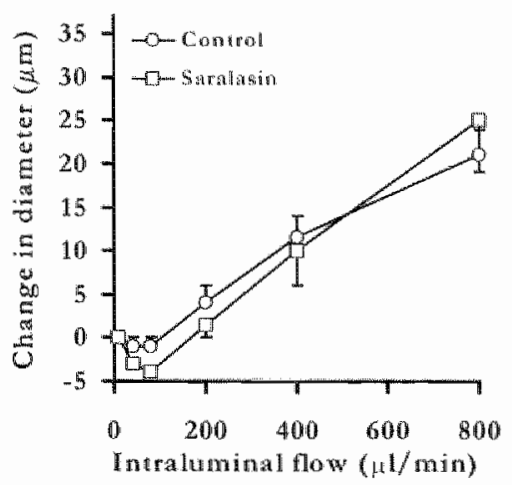

Figure 2.2. Changes in diameter ( $\mu \mathrm{m})$ following step-increases in intraluminal fow in catorid atteries from $\mathrm{TK}^{+/ 3}$ (efts closed symbols; $\mathrm{n}=7$ ) and $\mathrm{TK}$ (right; open symbols; $\mathrm{n}=6$ ) mice. Experiments were performed under control condision (wrles) or in the presence of suralasin (squars). * $P<0.05$ versus con

Interestingly, the $\mathrm{AT}_{2}$ receptor blocker $\mathrm{PD} 123,31.9$ significantly blunted the response to flow at high flow rates in $\mathrm{TK}^{+/+}$arteries exposed to losartan (Figure 2.3, leff). However, PD123,319 had no significant effect on FID in TK $/$ arteries exposed to losartan (Figure 2.3, rigbt). In $\mathrm{TK}^{+/+}$arteries, blockade of $\mathrm{AT}_{2}$ receptors with $\mathrm{PD} 123,319$ alone significantly reduced FID (from 200 to $800 \mu \mathrm{L} / \mathrm{min}$, Figure 2.4). Interestingly, the magnitude of dilatation of $\mathrm{TK}^{+/ *}$ arteries exposed to PD123,319 was comparable to that of TK arteries in the absence of any antagonists (Figure 2.4). Furthermore, PD 123,319 thad no significant effect on FID of TK arteries (Figure 2.4).

The bradykinin $B_{2}$ receptor antagonist HOE-140 also decreased FID in "TK"/ arteries confirming previous observations. ${ }^{37} \mathrm{~A}$ combination of both PD123,319 and HOE-140 significanty altered FID compared to control conditions (Figure 2.4). However, the impaired FID in the presence of both antagonists was not different from the FID observed in the presence of PD 123,319 alone.

FID was also significantly reduced in carotid arteries of $\mathrm{AT}_{2} /$ mice when compared to their wild-type littermates (Figure 2.5). In addition, HOE-140 significantly blunted HID in $\mathrm{AT}_{2}^{+{ }^{+}}$, but had no significant effect in $\mathrm{AT}_{2} \%$ mice (Figure 2.5). Dilatation to exogenously added bradykinin was investigated in perfused $(40 \mu \mathrm{L} / \mathrm{min})$ mesenteric resistance anteries $(\mathrm{n}=6)$ of $\mathrm{AT}_{2}{ }^{+/ t}$ and $\mathrm{AT}_{2} \%$ mice. There was no significant difference in pD2 values $(7.89 \pm 0.31$ versus $8.18 \pm 0.33)$ and the maximal responses $(52 \pm 12 \%$ and $47 \pm 7 \%$ ro bradykinin between $\mathrm{AT}_{2}^{+/ 4}$ and $\mathrm{AT}_{2} /$ mesenteric arteries, respectively. 
$\mathbf{T K}^{+/+}$

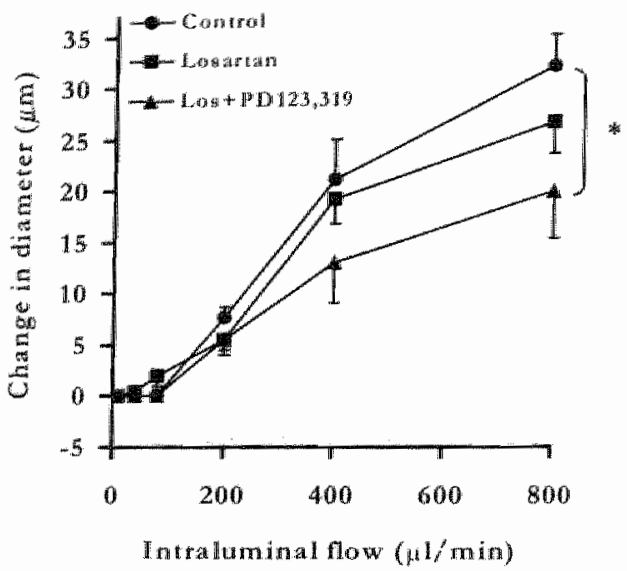

$\mathbf{T} K^{\mu}$

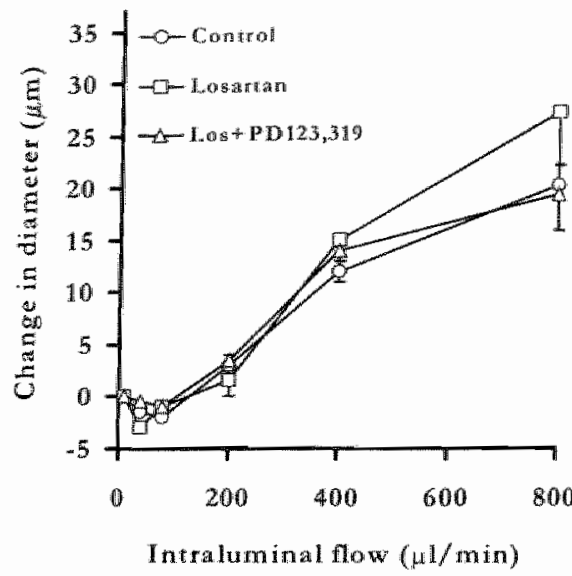

Figure 2.3. Changes in diamerer ( $4 \mathrm{~mm}$ ) followwing step-increases in intraluminal flow in carorid arteries from

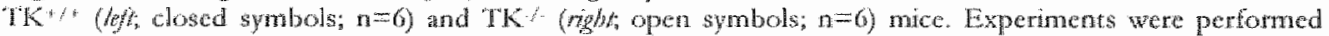
under control condition (cimles), in the presence of losattan (squares), or in the presence of both lasartan and PD123,319 (triangles). Control experiments and experiments with losartan were performed on carotid arteries from the same mice, while experiments with both losartan and PD123,319 were performed on carotid arteries of a different set of animals. ${ }^{*} P<0.05 . \mathrm{pD} 2$ values $(7.89 \pm 0.31$ versus $8.18 \pm 0.33$ ) and the maximal tesponses $(52 \pm 12 \%$ and $47 \pm 7 \%)$ to bradykinin betwen $\mathrm{AT}_{2} / 4$ and $\mathrm{AT}_{2} /$ mesenteric arteries, respecrively.

$\mathrm{TK}^{+/+4}$

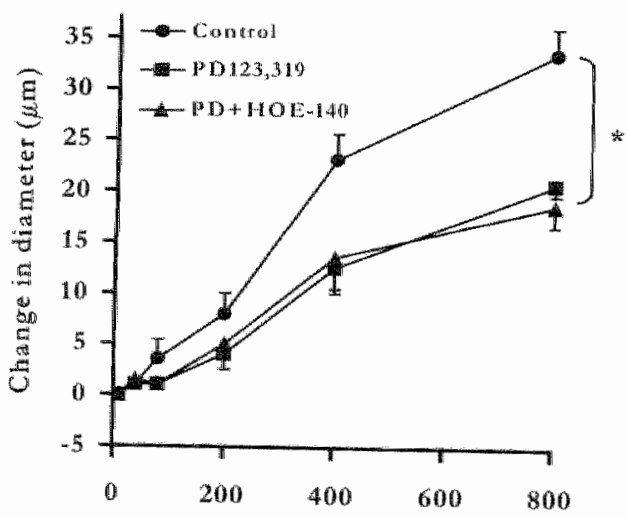

Intraluminal flow $(\mu \mathrm{l} / \mathrm{min})$
TK\%

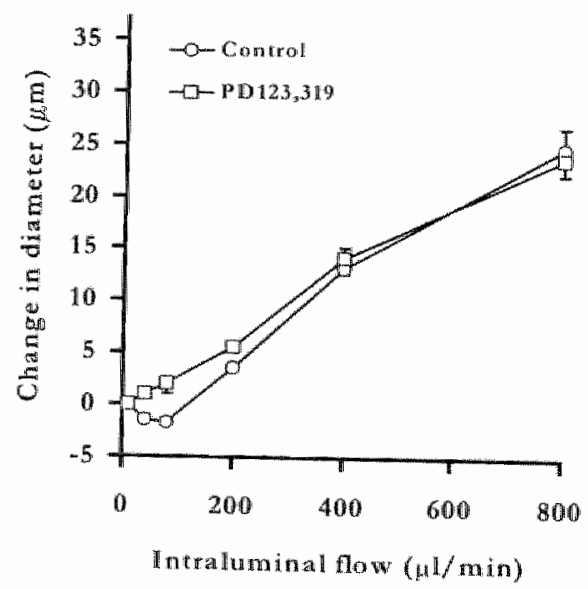

Figure 2.4. Changes in diameter (hm) following step-increases in intraluminal tow in carotid arteries from

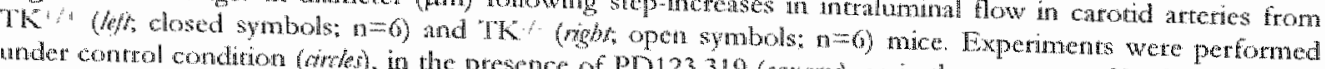

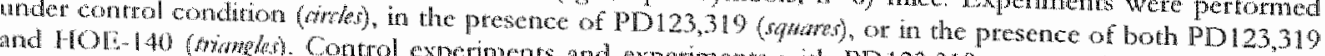

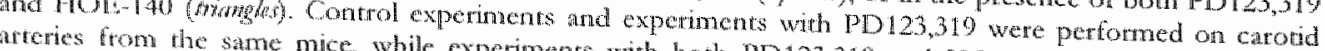
carosid arretics of a different set of animals $* P<0,05$. 

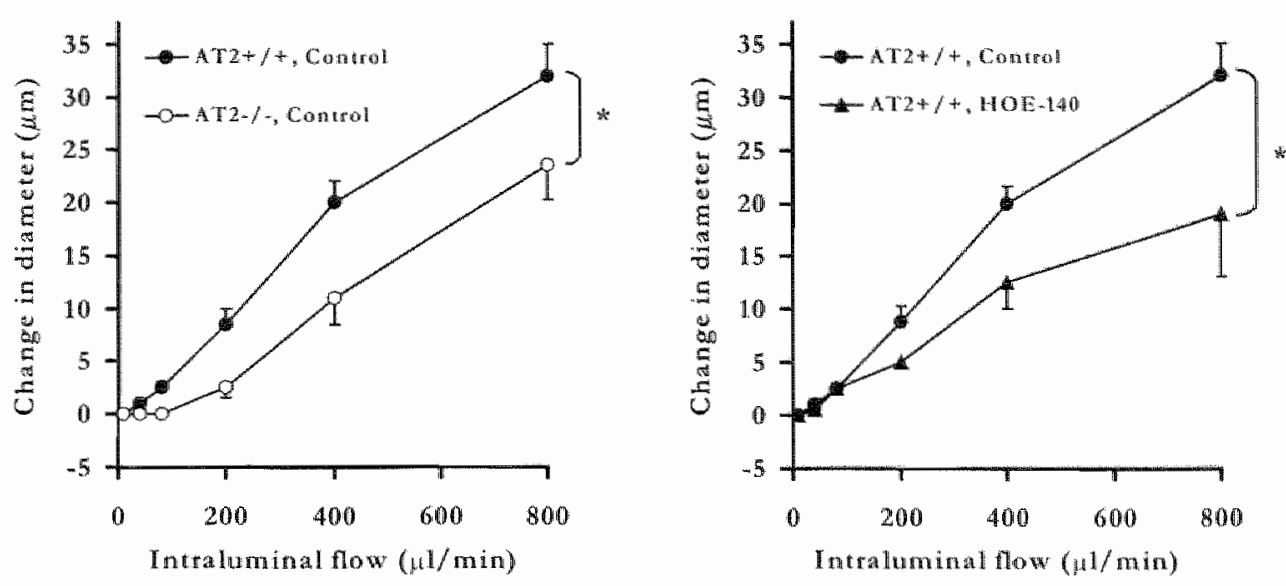

Figure 2.5. Changes in diameter $(\mu \mathrm{m})$ following step-increases in intraluminal flow in catrotid atterics from $\mathrm{AT}_{2}+\%$ (closed symbols; $n=7$ ) and $\mathrm{AT}_{2} \%$ (open symbols; $\mathrm{n}=6$ ) mice. Fipperimens were pertomed under control condition (arder) or in the presence of HOE 140 (raftawe) in catrodid arteries from the same mouse. $* p<0.05$

\section{Discussion}

Tissue kallikrcin activation contributes to flow-induced dilatation by activating bradykinin $\mathrm{B}_{2}$ receprots coupled to endothelial NO release. ${ }^{37,176,197,360,469}$ In this study we demonstrate that angiotensin $\mathrm{AT}_{2}$ receptors mediate the tissue kalliktein-dependent dilatation induced by shear stress in murine carotid arteries.

Before investigating the putative contribution of $A T_{2}$ receptors in flow-induced dilatation mediated by the endogenous kallikrein-kinin system, we first verified the presence of $\mathrm{AT}_{2}$ receptors in carotid arteries, because these receptors are either absent or expressed at a low level in blood wessels from adult animals. ${ }^{251}$ RT-PCR experiments demonstrated the presence of $A T_{2}$ receptor mRNA in carorid arteries from wild-type and mice lacking tissue kallikrein (TK/). In perfused carotid arteries we observed that flowinduced dilatation was significantly smaller in TK/ compated to TK+tmice, confirming our previous study ${ }^{37}$ The first hint regarding the involvement of angiotensin receprors in flow-induced dilatation mediated by endogenous kinins came from experiments with satalasin, a nonspecific $A T_{1}$ and $A T_{2}$ receptor antagonist. Satalasin impaircd the flowinduced dilatation in $\mathrm{TK}^{+/+}$arteries, but not in $\mathrm{TK}$ arteries, which are unable to generate endogenous kinins, 37,359

To further determine which of the angiotensin recepror is involved in flow-induced dilatation coupled to the vascular kallikrein-kinin system, we studicd the effect of flowinduced dilatation of selective antagonists to either $A T_{1}$ or $A T_{2}$ receptors. Neither losartan nor candesartan modified flow-induced dilatation in both " $T K^{+/ t}$ and $T K$ / micc, demonstrating that $\mathrm{AT}_{1}$ receptors do not contribute to the kinin-mediated dilatation induced by shear stress. This incerpretation is in agreement with other studics showing absence of $A T_{1}$ receptor antagonism in flow-induced dilatation, ${ }^{35.3}$ but it may contrast with previous studies showing that angiotensin II increases cyclic GMP levels by 
activating an endothelial $A T$ receptor, ${ }^{57,7}$ The apparent discrepancy might result from the absence of shear stress and the use of high concentrations of exogenous angiotensin II in these studies.

Unlike the blockade of the AT" receptor alone, exposing arterics to antagonists of both AT receptor subypes decreased the Alow-induced dilatation in TK" ${ }^{*}$ arteries, thus implying that $A T_{2}$, but not $A T_{1}$ receptors, contribute to flow-induced dilatation in wildrype arteties. The inhibitory effect of PD123,319 on this response in wild-type arteries reinforce his interpretation. Interestingly, A 12 receptor antagonism reduced flow-induced dilatation in wild-type mice to reach comparable levels to those observed in $T K /$ mice in the absence of any antagonists. The effect of $A T_{1}$ and $A T_{2}$ receptor antagonists was also examined in $\mathrm{TK}^{-}$arteries. Unlike $\mathrm{TK}^{++}$arteries, blockade of $\mathrm{AT}$ and/or $\mathrm{AT}_{2}$ teceptors did not modify flow-induced dilatation in $T K$ arteries. As TK arteries express both $\mathrm{AT}_{2}$ and $\mathrm{B}_{2}$ receptots, ${ }^{37}$, whin wurly we conclude that the participation of $A T_{2}$ receptors to flow-induced dilatation requires the presence of a functional kallikrein-kinin system.

We further confirmed the results obtained with saralasin and PD123,319 in wild-type mice by investigating flow-induced dilatation of carotid arteries obtained from $\mathrm{AT}_{2}$ neceptot deficient mice $\left(\mathrm{AT}_{2} /\right)^{-252}$ Flow-induced dilatation was blunted in carotid atteries of these mice compared to arteries of wild-rype mice. In addition, $B_{2}$ receptor antagonism reduced fow induced dilatation in $\mathrm{AT}_{2}^{* / *}$, but not in $\mathrm{AT}_{2} /$ mice, although deletion of the $\mathrm{AT}_{2}$ receptor gene did not affect the functional response to $\mathrm{B}_{2}$ receptor activation with exogenous bradykinin. Taken together, these findings indicate that the participation of bradykinin $B_{2}$ receptors in flow-induced dilatation requires the presence of functional A T.2 receptors.

We next studied the possible contribution of bradykinin $B_{2}$ receptors to the $A T_{2-}$ dependent flow-induced dilatacion in wild-type mice. Antagonism of the $\mathrm{B}_{2}$ receprot did not further decrease flow-induced diatation in wild-type arteries alteady exposed to $A T_{2}$ receptor blockade. Previous results demonstrated that $B_{2}$ receptor blockade in these arteries significandy blunted flow-induced diatation. ${ }^{37}$ Taken together, these obserwations suggest that there is no additional inhibitory effect on flow-induced dilatation by AT2 $_{2}$ roceptor antagonism when $B_{2}$ receptors are blocked or the kallikrein-kinin system is inactivated. Similarly, if $A T_{2}$ teceptors ate blocked or not expressed, $B_{2}$ teceptor blockade no longer inhibits flow-induced diatation. Thus, the present data demonstrate that the involvement of $\mathrm{AT}_{2}$ receptors in flow-induced dilatation requites the presence of both functional badykinin $B_{2}$ receptors and an activ kallikrein-kinin system. Furthemone, this study supports the conclusion that the fow-induced dilatarion mediated by the local kallikrein-kinin system requires the presence of functional $\mathrm{AT}_{2}$ receptors. This interpretition is in agteement with previous studies indicating that $A T_{2}$-dependent dilatation, following exposure to exogenous angiotensin $I$, involves bradykinin $B_{2}$ receptor activation. ${ }^{189,28,4}, 493$

Although the exact mechanism linking $A T_{2}$ and $B_{2}$ receptors in flow-induced difatation remains to be elucidated, several hypotheses can be stated. The increase in shear stress may favot and augment the boundary layer mass transport of kinins and angiotensin II to their teceptors therefore decreasing their degtadation rate. ${ }^{130,364}$ However, we cannot exclude the possibility that shear stress might also modulate endogenous pepride synthesis. Indeed, as a short-tem increase in sheat stress enhanced ACE activity, Gow 
simulation may augment the local production of angiotensin II. 451 However, an increase in $A C E$ activicy would also contribute to a enhanced degradation of locally formed kinins, ${ }^{29}$ thus counterbalancing the effect of an augmented angiotensin II synthesis. Interestingly, the lack of an additional effect of $A T_{2}$ and $B_{2}$ receptor blockade supports the conclusion that these two teceptors do not act synergistically. This observation also fawors the interpretation that these rwo recetor pathways lie upstream or downstream from ach other. Activarion of the $\mathrm{AT}_{2}$ pathwa might precede stimulation of endogenous kinin synthesis and $B_{2}$ receptor actiwation, as indicated in a recent study. interpretation is also supported by data from this study and previous work as the dilatation in response to bradykinin was unchanged following inacrivation of either kallikrein or $A T_{2}$ receptors. Furthemore, overexpression of $A T_{2}$ receptors in vascular smooth muscle cells increases intracellular acidosis, resulting in an enhanced kininogenase acrivity and subsequent kinin generation. 55 However, activation of kininogenase(s) by lowering intracellular $\mathrm{pH}$, following $\mathrm{AT}_{2}$ receptor activation, is not fully compatible with the known in who chatacteristics of tissue kallikrein activation. ${ }^{4+1}$ Altematively, the present results may suggest a direct molecular interaction between $B_{2}$ and $A T_{2}$ receptors, comparable to the one recently proposed for $A T_{1}$ and $A T_{2}$, but these different hypotheses would require further investigations.

In conclusion, the present study demonstrates that flow-induced dilatation mediated by the vasculat kallikrein-kinin system tequires both $A_{2}$ and $B_{2}$ receptors. This pathway would contribute to the regulation of wascular tone by offsetting vasoconstrictor and pro atherogenic effects of angiotensin II under physiological shear stress values in the vessel wall.

\section{Acknowledgements}

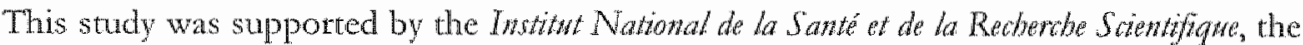

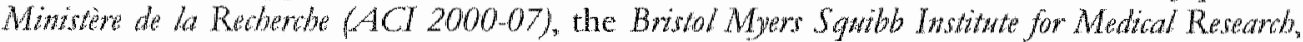
and the Gronpe Paris-Nord de Redenthe Pabologw Varnhane. S. Betgaya was supponted by a fellowship from the Fondation pour la Recherbe Mediale and R. Hilgers by a Marie Cario dorom stipund from the Enropean Commumity. We would also like to thank Eric Mathicu for his technical assistance. 

Chapter 3

\section{Role of Tissue Kallikrein During Arterial Remodeling in Response to Altered Blood Flow}

Sonia Bergaya, Rob H.P. Hilgers ${ }^{2}$, Paul MH. Schiffers², Gregorio F. Fazzi?

Pierre Meneron', Bernard I. Lévy', Jo G.R. De Mey², Chantal M. Boulanger

"Institut Narional de la Santé et de la Recherche Médicale (TNSERM), Unit 541, Hôpital Larboisicte, Paris, France,

2Cardionascular Researth Institute Maastricht (CARM), Universiteit Mastricht, the Netherlands,

TNSERM, Unit 367 , Paris, France.

Submitted 


\begin{abstract}
Objective. The tisue kallkrem-kimin system (KKS) participates in acure arterial responses to changes in Alow. Thus, the aim of this study was to elucidate the potental role of the vasculat $\mathrm{KKS}$ during arterial remodeling in response to chronic changes in biood flow.

Methods and Results. We ligated the left common carotid artery of mice lacking the tissue kallkrein gene (TK-) and their wild-rype littemates (TK $\%$ ). Sham-operated mice were used as controls (normal fow). Pressure-diameter curves were obtained in isolated cantrid arteries 4 weeks after surgery. In the ligated group, carotid artery blood Aow was significantly higher in the right carotid artery (high flow) than in the left carotid artery (no flow) and in nomal flow carotid attery. Nomal flow carotid arteries wene smallet in $\mathrm{TK} \%$ than in $\mathrm{TK}^{+/ 4}$ mice $(740 \pm 19 \mu \mathrm{m}$ versus $791 \pm 21 \mathrm{~mm}$ at $100 \mathrm{mmHg}$, respectrely). In both strains, dameter and structure were similat in nomal and high tow carotid artery. Diameters of no flow carotid artery were significanty reduced compared to normal flow carotid artery, being more pronounced in $T K \%$ mice $(\Delta 260 \mu m)$ than in $T K^{+/ *}(\Delta 179 \mu \mathrm{m})$. In both strains, unilateral catotid artery ligation tesulted in similar increases in media cross-sectional area without changes in collagen and elastin contents.

Conchision. The vasculat $K K S$ seems to protect against excessive inward remodeling in response to flow cessation. Moreover, mechanisms involved in acute vasomotor responses to shear stress likely differ from those operating in structural responses to chronic changes in blood flow.
\end{abstract}




\section{Introduction}

Tissue kallikrein is a serine protease expressed in the vascular wall that cleaves kininogen to generate kinins, 359,397 In endothelial cells, kinins stimulate the telcase of nitric oxide $(\mathrm{NO})$ and prostacyclin $\left(\mathrm{PGI}_{2}\right)$ through the activation of bradykinin $\mathrm{B}_{2}$ receptors. NO and $\mathrm{PGI}_{2}$ exert relaxing, antaptolifetative, and antimigtatory effects on vascular smooth muscle cells. ${ }^{70,177,463,70}$ Furthermore, the vascular kalliktein-kinin system (KKS) participates in flow-induced dilatation (FID) through the activation of bradykinin B receptors coupled to endothelial NO release.".

Wall shear stress is a major determinant of the structural arterial lumen dianeter and, indirectly, of atterial. wall mass. $184,185,308,4,34,533$ In response to changes in shear stress, endothelial cells modulate their release of vasoactive mediators and alter their pattern of gene expression. ${ }^{110}$ Arterial structural responses to changes in blood flow have been suggested to be endothelium-dependent ${ }^{3 / 2}$ and to display similatities to acure flowinduced dilatation. $4.5 \mathrm{In}$ this regard, endothelial NO synthase (eNOS) appears to be a major determinant of flow-related arterial remodeling, ${ }^{46,5,47}$ Indeed, lack of eNOS leads to smooth muscle cell hypertroply in response to a drastic chronic decrease in blood flow. Kinins, which can stimulate NO release, also appear to contribute to arterial remodeling, since bradykinin $B_{1}$ and $B_{2}$ receptors participate in the inhibition of neointima formation caused by either ACE inhibition or overexpression of the human tissue kallikrein (TK) gene in experimental models of altered blood flow. $137,138,380$

Because TK is involved in acte NO-mediated flow-induced dilatation and because of the putative role of kinins in arterial remodeling, we investigated whether deficiency of TK affects atterial remodeling during chronic alterations in blood flow. We therefore performed unilateral carotid artery ligations, ${ }^{300}$ resulting in a cessation of blood flow in the ligated and a doubling of blood flow in the contralateral (righr) carotid arteries, in tissue kallikrein deficient $(\mathrm{TK} /)$ and wrild-type $\left(\mathrm{TK}^{+/}\right)$mice. We focused our attention on the central part of the arteries where no signs of neointimal formation developed. ${ }^{211}$

\section{Materials and Methods}

\section{Animzals}

Tissue kallikrein deficient (TK/) mice were obtained by targeted distuption of the "TK. (k/kt) gene by replacing 100 bp of exon 4 with the neomycin-resistance gene in embryonic stem cells as described eatlier. ${ }^{3+4}$ Breeding of "T"K" mice derivec from these embryonic stem cells led to $\mathrm{TK}^{+/ t}$ and $\mathrm{TK} \%$ littermate mice. Experiments were performed on 12-week old male mice with a mixed genetic backgtound (129/SVC57BI/6J). To identify the genotype, DNA was extracted from the tall, and the presence of targeted TK alleles was detected by RT-PCR.

\section{Unilateral Carodid Antery Ligation}

Male TK $\%$ and TK mice ( 3 to 4 months old) were used in all experiments. The animals were anesthetized with a gas mixture $\left(\mathrm{O}_{2} /\right.$ isoflurane). Carotid anteries were carefully exposed by a midline incision $(1 \mathrm{~cm})$ in the neck of the mouse. The left common cafotid 
artery was dissected in situ and ligated near the carotid bifurcation with a 5.0 surgical silk suture as previously described. 3 . Since mouse carotid arteries lack side branches, left carotid arteries ligated near the bifurcation will have no net forward flow and will be referred to as "no flow", while the ipsi- or contralateral (right) carotid artery will be referred to as "Tigh flow", In parallel, carotid arteries of sham-operated mice will be teferred to as "normal Alow". All surgical procedures were in accordance with the Eutopean Communiry guidelines conceming the care and use of laboratory anmals (Ministere de l'Agriculture, France, atuthotzation 07430).

\section{Canolid Artery Bhod Flow Measurenzents}

Four weeks after surgery, both carotid arteries were carefully dissected before measuring in sith blood low. We used a transit-time ultasonic flow probe $(0.5$ mm $V$ series, Transonic Systems) that was mounted on a micromanipulator and positioned halfway between the aotric arch and the carotid artery bifutcation. Mean BF was obtained by averaging during a 5 minute period for the left and right catotid artery of each animal.

\section{Pressure-Dianueder Curves}

Catotid artery segments $(5 \mathrm{~mm}$ long, halfway between the aortic arch and the carotid bifurcation) wete isolated and placed in ice-cold calcium-free modified Krebs-Ringer buffer (composition in mmol/L: $144 \mathrm{NaCl}, 4.7 \mathrm{KCl}_{3} 1.2 \mathrm{MgSO}_{4}, 1.2 \mathrm{KH}_{2} \mathrm{PO}_{4}, 14.9 \mathrm{HEPES}, 2.5$ $\mathrm{CaCl}_{2}$, and $5.5 \mathrm{glucose}$ pH 7.4). The proximal end of each vessel was cantulated on a glass cannula in an organ chamber (arteriograph system, Iving System Instrumentation, Burlington, VT, USA) and the distai part of the segment was closed with a nylon knot. The organ chamber was flled with calcium-free Krobs-Ringer buffer containing $10 \mu \mathrm{mol} / \mathrm{L}$ sodium nitroprusside (SNP) to assure maximal vasodilatation. The cantulated arterial segment was pressurized using a feedback-controlled pressure source (Tiving Systems Instrumentation) and placed under a binocular lens connected to a video camera for recording of arterial diameter. Due to the intransparancy of the carotid attery, outer diameters were recorded.

A pressure-diameter $\left(\mathbb{P}^{3}\right.$-D) rehationship was established by tecording the diameter during stepwise (10 mmHg) increases in intraluminal pressures from 20 to $150 \mathrm{~mm} / \mathrm{g}$. After each step the vessel was allowed to equilibrate until a stable dameter was reached. Afret the experiment, rhe arteries were fixed at ansmural pressute of $100 \mathrm{mmHg}$ in $4 \%$
phosplate-buffered formaldehyde and stored in $70 \%$ ethanol.

\section{Mophowitom}

Fixed vessels were embedided in paraffin and cross-sections (4 $4 \mathrm{~m}$ ) were stained with Lawson's solution (Boom, Meppel, the Netherlands) to visualize the internal and external clastic lamina. In the case of carotid artertes the morphometric analysis focussed on the central part of the vessel, halfway berween the aortic atch and the catotid bifurcation. Unlike the distal part near the ligation, the central part showed no signs of neointima formation or inflammation. Video images were made from cross-sections using a Zeiss axioscope and a standated charge-coupled device camera (Sony). Using IAVA softwate (Sigma Scan, Jandel Scientific, Corte Madera, CA, USA) the circumference of the internal and external elastic laminae were detemined. From these values the media cross-sectional 
area (mCSA) was calculated. The average number of medial nuclear profiles per crosssection was measured by counting the nuclear profiles in the smooth muscle layers of hematoxylin-eosine stained cross-sections ( $\mathrm{n}=3$ for each artery and individual mouse). Digital images of Lawson stained cross-sections were made at $400 \mathrm{X}$ maginification. Using Leica QWin softwate the density of elastin was determined in two areas of the media and averaged. Elastin content was calculated by multiplying elastin density with the medial cross-sectional area. To stain collagen, cross sections were deparaffinised and incubated in phosphomolybdenic acid $\left(0.2^{\%} \%\right.$ for 5 minutes, followed by incubation with Sirius Red $(0.1 \%)$ in saturated picric acid solution for 90 minutes. After washing with $0.1 \mathrm{MHCl}$ for 2 minutes, sections were dehydrated and protected with coverslips. The densiry and content of collagen in media were quantified as described for elastin.

\section{Data Analysis and Statititics}

Results are expressed as mean \pm SEM of $n$ experiments, $n$ represents the number of animals used for each experimentad protocol. Statistical evaluation was performed by ANOVA for factorial or repeated measurements, followed by Scheffe $t$ test. ${ }^{575}$ Values of $P<0.05$ were considered statistically significant.

\section{Results}

\section{General Obsentations}

Body weights did not differ berween the four groups of mice. In the sham groups, carotid arterial blood flow (mean of left and right artery) did not differ significantly between $\mathrm{TK}^{+/ *}$ and $\mathrm{TK} /$ mice $(0.69 \pm 0.20 \mathrm{~mL} / \mathrm{min}$ versus $0.59 \pm 0.08 \mathrm{~mL} / \mathrm{min})$. In both groups of mice, left carotid artery ligation resulted in a cessation of blood flow in the occluded antery and a comparable substantial increase of blood flow in the right carotid artery $(1.25 \pm 0.20$ $\mathrm{mL} / \mathrm{min}$ in $\mathrm{TK}^{+/+r}$ mice and $1.28 \pm 0.23 \mathrm{~mL} / \mathrm{min}$ in $\mathrm{TK} /$ mice).

Arterial Structure of TK" and TK- Mice

Outer diameters of pooled left and right carotid arteries wete significantly smaller in TK $\%$ compared to $\mathrm{TK}^{+/}$sham-operared mice (Figure 3.1). However, media thickness (not shown) and media cross-sectional area were comparable in $\mathrm{TK}^{* /+}$ and $\mathrm{TK} /$ mice (ligute 3.1 , insent). There was no difference in collagen and elastin content and density in carotid arteries of both strains (Table 3.1).

\section{Carotid Arteo Strutural Changes in Response to Altered Blood Flow}

In both $\mathrm{TK}+1+$ and $T K \%$ mice, outer diameters of high flow arteries wete not different from normal tlow arteries (Figure 3.2). Medial cross-sectional area, collagen and elastin were not significantly altered in carotid arteries of both strains exposed to elevated blood flow (Figure 3.3, Table 3.1). 


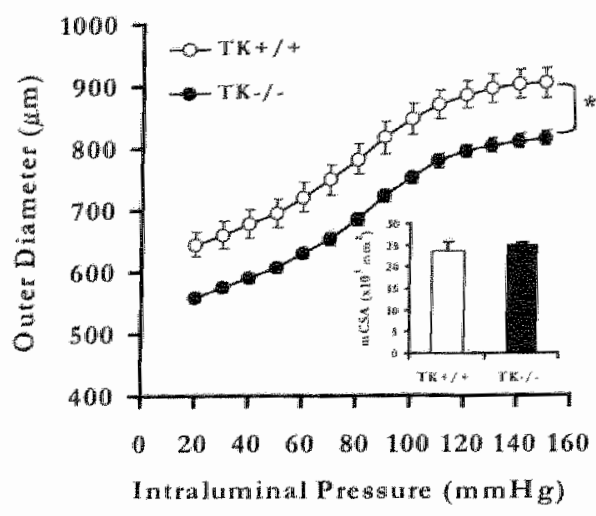

Pigure 3.1. Relatons befwen distending intratuminal pressure ard outer diameter in isolated catrotal arteries (poled lete and right) of TK (open symbols) and TK (niled symbols) mice. Lurm: media CSA of tsolated carotid arteries (pooled lof and right) of $\mathrm{TK}$, (open bars) and $\mathrm{TK}$ (filled barsy mice. P<0.05 between the two growps of mice.

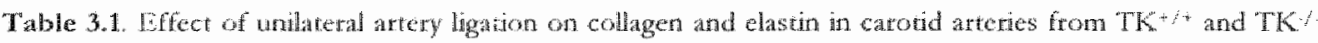
mics.

\begin{tabular}{|c|c|c|c|c|c|}
\hline & Strain & Unit & Normal Flow & High Flow & No Flow \\
\hline Collagern & $\begin{array}{l}\mathrm{TK} \\
\mathrm{Th}\end{array}$ & $\begin{array}{l}0 / 0 \\
x 10^{3} \mathrm{Lnn}^{2} \\
6 / \mathrm{m} \\
\mathrm{x} 10^{3} \mathrm{Hm}^{2}\end{array}$ & $\begin{array}{r}35.7 \pm 6.7 \\
9.8 \pm 1.3 \\
36.4 \pm 4.0 \\
9.8 \pm 0.8\end{array}$ & $\begin{array}{l}37.2 \pm 8.9 \\
10.5 \pm 3.0 \\
34.7 \pm 9.5 \\
10.1 \pm 2.5\end{array}$ & $\begin{array}{l}15.1 \pm 20 \% \\
6.5 \pm 1.0 \\
10.1 \pm 3.0 \\
4.3 \pm 1.9\end{array}$ \\
\hline Elastin & 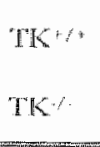 & $\begin{array}{l}6 / \% \\
\times 10^{4} \mathrm{Lm}^{2} \\
6 / 0 \\
\times 10^{4} \mathrm{Hm}^{2}\end{array}$ & $\begin{array}{l}38.1 \pm 1.9 \\
10.4 \pm 0.8 \\
36.0 \pm 2.5 \\
9.9 \pm 0.7\end{array}$ & $\begin{array}{l}41.9 \pm 5.6 \\
10.8 \pm 1.1 \\
38.6 \pm 1.4 \\
10.3 \pm 0.6\end{array}$ & $\begin{array}{l}21.2 \pm 4.0 * .2 \\
8.4 \pm 1.3 \\
280 \pm 6.7 \\
11.6 \pm 2.1\end{array}$ \\
\hline
\end{tabular}

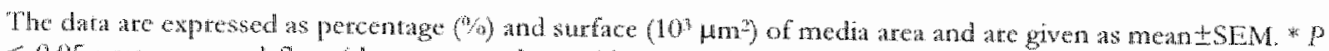

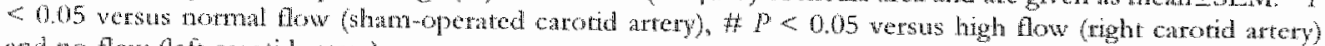
and no-flow (kefi carovid artery).

Ligation of the left catotid artery resulted in a significant reduction of the outer diameter and discensibility (Figure 3.2). The observed inward remodeling was more pronounced in ligated arteries of TK mice $(811 \pm 11$ to $553 \pm 17 \mu \mathrm{m} ;-32 \%$ ) than in TK / mice (from $899 \pm 23$ to $734 \pm 31 \mu \mathrm{m} ;-18 \%$ ). In both strains of mice, ligation of the left carotid artery resulted in a significant medial hypertrophy, which was not significantly different between TK+/t and TK/ mice (Figure 3.3). In TK" mice, unilateral catodd artery ligation decreased both elastin and collagen density in the no flow artery without altering the contents of these extracellular matrix proteins (Table 3.1). However in TK-tmice, ligation decteased collagen density in the no flow artery, but did not significantly
affect elastin density (Table 3.1). 
$\mathrm{TK}^{+/+}$

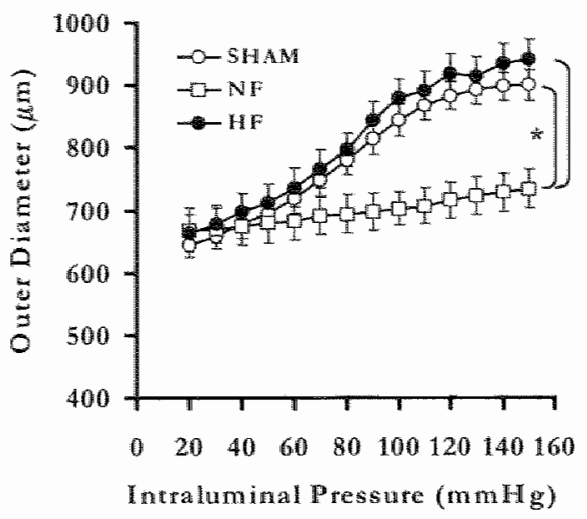

$\mathrm{TK}^{\%}$

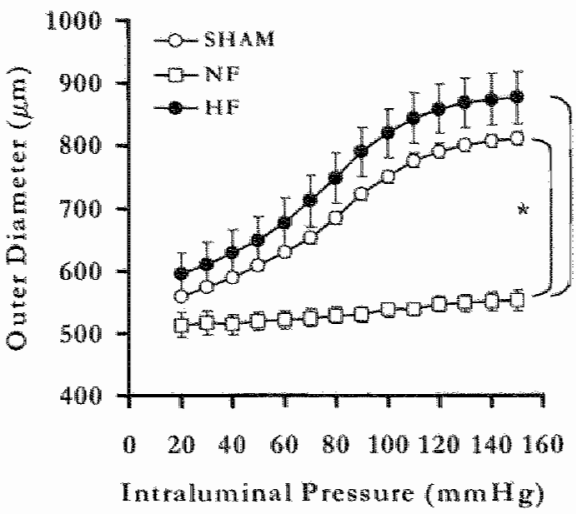

Figure 3.2. Fffecis of unilateral carotid arterg ligation on relations betwen distending intraluminal pressure

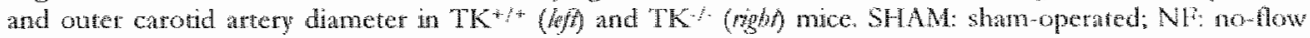
(Ggated) vessels; HF: high How (lipperperfused) vessels. * $P<0.05$ versus NF interics.
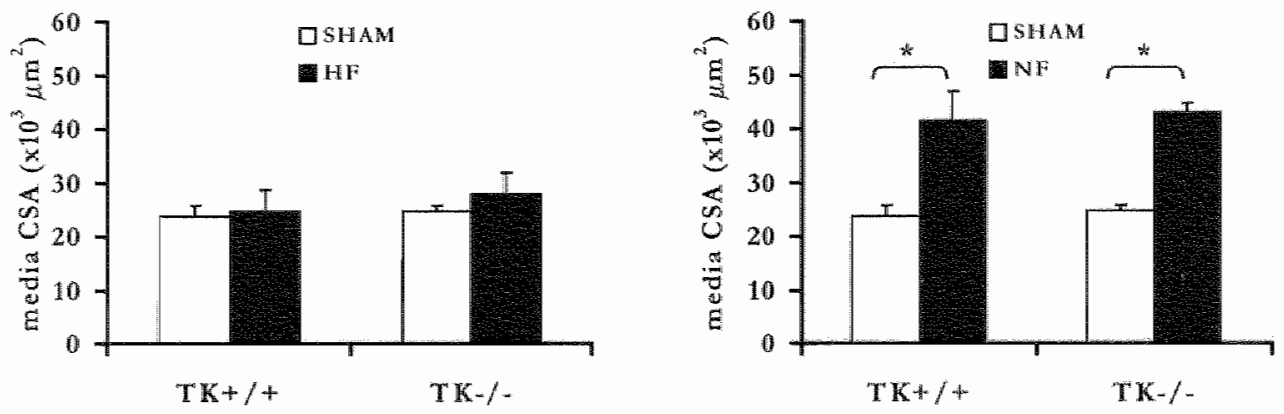

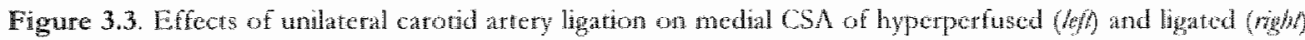

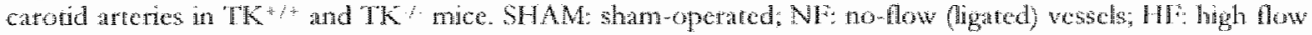
(hyperperfused) vessels. ${ }^{*}$ P<0.05 versus shatn, * PK0.05 versus SMAM. 


\section{Discussion}

The arterial wall expresses tissue kallikrein (TK), which contributes to flow-induced NOmediated dilatation. ${ }^{37}$ The present study shows that the vascular kallikrein-kinin system participates in the morphogenesis of large elastic arteries and limirs inward remodeling in flow-limited arteries. These results also demonstrate that the contribution of the kallikrein-kinin system to flow-induced remodeling unlikely involves similar pathways to those activated during flow-induced dilatation.

Local mechanical forces that govern arterial structure include circumferential wall stress (CWS) and wall shear stress (WSS). "10 Chronic changes of these forces result in arterial remodeling. $137,138,184,135,300,312,4,4,4,3,3,46,3,547,55,3$ These atterial adlaptive structural responses involve partly interrelated alterations in (a) arterial wall mass, (b) mechanical properties of the arterial wall material and (c) arterial lumen diameter. Because WSS can stimulate intra-arterial production of bradykinin, ${ }^{37}$ we compared flow-related arterial remodeling in large conduit arteries of $\mathrm{TK}^{\prime}$ and $\mathrm{TK}^{+/+}$mice using the unilateral carorid artery ligation method introduced by Kumar and Lindner. 300

The outer diameter of normal flow carotid arteries was significandy smaller in TK\% than in littermate wild-type mice over a broad pressure range. A thinner arterial wall in TK arteries can not explain the difference in ourer diameter, as media thickness was compatable between $\mathrm{TK}^{+/+}$and TK $/$mice. Since blood pressure and in wiro carotid artery blood flow were similat in both strains, ${ }^{37}$, this sndy the present results indicate that TK is involved in the development of the arterial lumen diameter during pre- and postnatal growth. They also suggest that the arterial wall of TK $\%$ mice may be chronically exposed to slightly higher shear stress values compared to carotid arteries of wild-type mice. This interpretation, together with the previously observed blunted flow-induced dilatation in TK $\%$ arteries, ${ }^{37}$ would suggest that the arterial wall was unable to normalize wall shear stress.

Ligation of the left common carotid artery was successful, since a complete cessation of blood flow was measured in the occluded arteries and a doubling of blood flow developed in the contralateral artery, whete, as a consequence, shear stress was augmented. Connections between the left and right carotid circulation, such as the circle of Wilis, form the anatomical basis for this shunting. We did not observe a significant outward remodeling of carotid arterics of both strains exposed to a 4 -week period of a doubling in blood flow. Flow-relared remodeling has been demonstrated to be age- and strain-dependent. Aging blunts ourwatd carotid carotid artery remodeling in rats ${ }^{361}$ and rabbits. "3to Hatmon and colleagues applied the unilateral ligation model in a variety of inbred strains of mice. They observed that carotid arteries of the $129 / \mathrm{SV}$ strain developed
the least outwatd remodeling in response to a doubling of blood flow 4-weeks post
ligation.21" Since the genetic background of the ming ligation.21" Since the genetic background of the mice used in this study is $129 / \mathrm{SV}$ $\mathrm{C} 57 \mathrm{Bl} / \mathrm{G}$ and the awerage age is 4 to 5 months, absence of significant outward arterial
remodeling might depend on these factors.

In carotid arteries of both strains, a chronic cessation of blood flow resulted in a compensatory inward arterial remodeling accompanied by a drastic reduction in arterial distensibility. These changes in arterial diameter were more pronounced in TK $\%$ than in $\mathrm{TK}^{+/}$mice, while the increase in media cross-sectional area was comparable between the 
two strains. The absence of an additional effect on medial hypertrophy in $\mathrm{TK}$ - mice in response to blood flow cessation is in agreement with studies investigating the effect of bradykinin $B_{2}$ receptor deficiency and adenovirus-mediated human tissue kallikrein gene delvery in the same model of unilatemal catotid artery ligation Considering the role of tissue kallikrein in shear stress-induced NO release ${ }^{3}$ and the role of NO as an negative regulator of vascular smooth muscle cell proliferation in response to a remodeling stimulus, ${ }^{* 3}$ absence of an additional effect on medial hypertrophy in TK\% mice is paradoxical. An explanation for this discrepancy might be the difference in the experimental model between the latter study (ligation of the external carotid artery) and the present study (ligation of the common carotid artery). One possible explanation is that the interruprion in blood flow observed in our study may decrease basal NO level to such wn exrent that an additional decrease in NO release, caused by a deficiency in endogenous kinins, would only have minor effects. Alternatively, other endothelimm-derived factors could compensate for the deficiency in NO release in $\mathrm{TK} \%$ carotid arteries, as suggested from a previous study investigating flow-related arterial remodeling in rats chronically weated with the NO synthase blocker L-NAME, The present sudy allso contrasts with tesults obtained in vimentin-deficient mice using the same surgical model ${ }^{5}$ Flowinduced dilatation is blunted in mesenteric ateries of these mice, 220 but inward remodeling was smaller and medial hypertrophy was larger compared to wild-type mice. 47 Taken together, these data suggest that the mechanisms implicated in acute and chronic responses are different. In this respect, the flow-related arterial aclaptations in $\mathrm{TK} / \mathrm{mice}$ likely involve different pathways than those mediating TK-dependent endothelial NO telease. ${ }^{37}$

It is not cleat which arterial component(s) is (are) cruciall in re-shaping the arterial architecture in response to chronic changes in blood flow. Since the medial cross-sectional area increase was comparable in carotid arteries of both strains after blood flow cessation despite a more substantial inward remodeling in TK $\%$ mice, indicates that the medial mass is not the main determinant. Since extracellular matrix proteins such as collagens and elastin determine atterial elasticity, we measured the contents and density of these proteins. Surprisingly, the marked medal hypertrophy and reduction in arterial distensibility resulting from blood low cessation were not accompanied by increases in either collagen or elastin content. Rather, the density of these extracellular matrix proteins was reduced. This seems largely due to the mediat hypertrophy but might also involve increased activity of matrix metalloproteinases (MMP-2 and -9) associated with smooth muscle cell migration, macrophage accumulation and neointima fomation in the proximal and distal (but not central) parts of the murine carotid artery exposed to blood flow cessation. ${ }^{172,173,187,260,253,313}$ If anything, collagen density dropped more and elastin density dropped less in the occluded TK/ carotid arteries, which cncroached mote on theit lnumen than occluded $\mathrm{TK} \mathrm{K}^{+/ 4}$ arteries. The greater narrowing of $\mathrm{TK} /$ arteries in response to blood flow cessation is in line with the reduced expansion during development described above. The vascular kallikrein-kinin system may thus be proposed to reduce inward arterial remodeling independent of collagen and elasin changes. Other extracellular matrix components such as frbronection ${ }^{52}$ and cytoskeletal proteins such as desmin merit attention in this respect. 
These results indicate that the vascular kallikrein-kinin system (a) is involved in the development of the arterial lumen diameter, and (b) reduces inward arterial temodeling in response to chronic feductions in blood flow. They also dissociate between short- and long-term arterial responses to shear stress.

\section{Ackenowledgement}

This study was supported by the Institut National de ta Santé et de la Recherche Scaentifique, an educational grant from the Groupe Paris-Nom de Recherche en Pathologie Vasculatre and by the Fondation povir la Restberche Médicale (Paris, France). 
Chapter 4

\section{Pregnancy-Related Arterial Structural and Functional Alterations Despite Blunted Flow- Induced Dilatation in Tissue Kallikrein- Deficient Mice.}

Rob H.P. Hilgers', Sonia Betgaya2, Paul M.H. Schiffers, Pieme Meneton", Chantal M Boulanger ${ }^{2}$, Daniel Hention², Benard I Lévy², Jo G.R. De Mey

'Cardiovasculat Reseatch Institute Maastricht (CARIM), Unversiteil Maastricht, the Nethetlands,

2Institut National de la Santé et de la Recherche Medicale (INSERM), Unit 541, Mopital Laribotsière, Paris, France,

TNSERM, Unit 367 , Paris, France.

Based on: Arterionder Thromb I asc Bial. 2003;23:1826-1832. 


\section{Abstract}

Objective. Tissue kallikrein (TK) participates in acute flow-induced dilatation (FID) of large arteries. We investigated whether TK-deficiency blunts FID and alters chronic flow-telated arterial changes in resistance-sized muscular arreries.

Methods and Results: Vasomotor responses and structural parameters were determined in uterine arteries isolated from nonpregnant, 18 to 19 days pregnant and 7 days postpartum $\mathrm{TK}^{\%}$ and $\mathrm{TK}^{+/ t}$ littermate mice. In $\mathrm{TK}^{\%}$, diameter, media cross sectional area $(\operatorname{CS} A)$, myogenic tone and dilatation in response to acetylcholine were comparable to those in TK ${ }^{+/+}$, but FID $(0-100 \mu \mathrm{l} / \mathrm{min})$ was significantly reduced ( $55 \pm$ $4 \%$ versus $85 \pm 4 \%$ in $\mathrm{TK}^{+/+}$). For both $\mathrm{TK} /$ and $\mathrm{TK} /+$, FID was not significantly reduced in the presence of $100 \mu \mathrm{M}$ L-NAME, but reduced to $50 \%$ in the presence of both L-NAME and $10 \mu \mathrm{M}$ indomethacin. In $\mathrm{TK}^{+/ t}$, pregnancy resulted in significant increases of diameter and CSA, and in the L-NAME-sensitive component of FID. In uterine arteries of pregnant TK $\%$, outward hypertrophic remodeling and the increase of FID wete similar to those in $T K+4$. By 7 days postpartum, uterine artery diameter and CSA did no longer differ from nonpregnant values and FID was markedly reduced in both TK\% and TK ${ }^{+/ 4}$.

Conchusions: These observations (1) confirm at the level of resistance arteries the key role of TK in FID and (2) indicate that TK deficiency and blunted FID do not comptomise the arterial remodeling and the changes in the contribution of nitric oxide to FID during and after pregnancy. 


\section{Introduction}

Tissue-kallikrein (TK) is a serine protease that generates kinins, such as bradykinin, by enzymatic cleavage of high and low molecular weight kininogens. 396.39 Bradykinim modulates vascular tone by srimulating vascular endothelial $B_{2}$ receptors and the subsequent release of $\mathrm{NO}$ and prostacyclin $\left(\mathrm{PGI}_{2}\right) .{ }^{60.89}$ Recently, Bergaya and colleagues demonstrated a reduced flow-induced dilatation (FID) in isolated carotid arteries of tissue-kallikrein-deficient (TK\%) mice, indicating a key role of the local kallikrein-kinin system in vasomotor responses of large arteries to increased shear stress. ${ }^{37}$

Pressure and flow are hemodynamic determinants of circumferential wall stress (CWS) and wall shear stress (WSS) in the arterial system. On an acute basis they modulate arrerial smooth muscle tone: an increase in transmural pressure triggers a myogenic contractile response, $\$ 3,156,157,464$ while an increase in flow induces an endotheliumdependent FID, $22,43,110,506$ Long-term changes in blood flow lead to arterial structural adaptations in order to notmalize WSS. $33,398,43+, 479,547,556$ In general, chronic blood flow elevations lead to a widening of lumen diameter, whereas sustained reductions in blood flow lead to a natrowing of lumen diameter. These structural atterial adaptations have been shown to be endothelium-dependent. ${ }^{312}$

Pregnancy may be considered a model of physiological flow-related remodeling of uterine atteries. It has previously been studied in rats, ${ }^{406}$ guinea pigs, ${ }^{426}$ and ewes. ${ }^{168}$ During pregnancy, blood flow through the uterine circulation increases substantially, 10,335 In order to accommodate this increase in uterine blood flow, the uterine vasculature undergoes luminal expansion and an increase in wall mass, , the a process termed outward hypertrophic remodeling or arteriogenesis. The increase in wall mass involves borh hypertrophy and hyperplasia of vascular smooth muscle cells as observed in the uterine artery of late pregnant rats. ${ }^{* 018}$ The driving force for this outwatd hypertrophic remodeling of uterine arteries during pregnancy is increased WSS. Previous studies have demonstrated that chronic elevations in blood flow lead to an upregulation of FID, mainly due to enhanced production of endothelial factors, such as $\mathrm{NO}$ and prostaglandins. 1 or instance, chtonic exercise in rats resulted in increased FID and expression of endothclial nitric oxide synthase (eNOS), ${ }^{200}$ and arterial wall mass increases in the arteriolar network in skeletal muscle. ${ }^{488}$ These observations suggest that long-term elevations in blood flow increase acute FID, but do not explain whether FID is required for successful structural remodeling of arteries.

The purpose of this study was to investigate whether TK-deficiency alters $\mathrm{F} \mid \mathrm{D}$ and chronic flow-related arterial structural and functional alterations in resistance-sized musculat arteries. Vasomotor responses and structural parameters were determined in uretine anteries isolated from nonpregnant, 18 to 19 days late pregnant and 7 -days postpartum $\mathrm{TK}$ and $\mathrm{TK}^{+/+}$mice. 


\section{Methods}

\section{Mice latking tissue kalliketip.}

Tissue kallikrein (TK)-rull mice were obtained by targeted disruption of the TK kble/ gene that was accomplished by replacing 100 bp of eron 4 with the neomycin-resistance gene in embryonic stem cells. ${ }^{359}$ Breeding of heterozygous mice derived from these embryonic stem cells led to wild-type (TK ${ }^{+/}$), heterozygous $\left(\mathrm{TK}^{+/}\right.$) and homozygous (TK/) littermate mice. Mice had a mixed genetic $129 / \mathrm{SV}$-C57BL/6 backgtound.

At an age of 15 wecks, four to frve $\mathrm{TK}^{+/+}$female virgin mice were placed together in a cage with one male TK $/$ mouse. Female TK $/$ mice were put together with one male $\mathrm{TK}^{+/+}$mouse. This allowed to compare pregnant $\mathrm{TK}^{\%}$ and $\mathrm{TK}^{+/+}$mice, each carrying heterozygous feruses. Males were separated from the females after 24 hours. When mating was successful, the day of placing a male mouse with the females was considered as day 0 . For the late pregnant group, pregnant mice were sacrificed 18 to 19 days after day 0 . For the postpartum group, mothers were sacrificed 7 days after delivery of their pups. For the nonpregnant group, age-matched (18 week-old) virgin mice were used.

\section{Preparation of biood wessels and experimental set up.}

Mice were sacrificed with an overdose of pentobarbital (i.p.). The mesentery and uterus were dissected and pinned out on a petridish (coated with a layer of silicon). Adipose- and connective tissue were carefully removed from the arteries. From every mouse one segment ( $3 \mathrm{~mm}$ ) of a first-order mesenteric artery and one segment ( $3 \mathrm{~mm}$ ) of the uterine artery at midpoint of the uterine arcade were isolated. Both preparations were mounted in an arteriograph system (I.jing System Instrumentation, Burlington, VT, USA), in which wall thickness and lumen diameter could be continuously monitored while controlling intraluminal pressure. Borh ends of the vessels were cannulated on 120-150 $\mu \mathrm{m}$ wide glass micropipettes (Living System Instrumentation, Burlington, VT, USA), and tied with two 17- $\mu \mathrm{m}$ thin nylon threads. The two micropipettes were selected such that both had the same resistance to how. Arterial segments were bathed in a $10-\mathrm{ml}$ organ chamber filled with physiological salt solution of the following composition (mmol/L): $144 \mathrm{NaCl}, 4.7$ $\mathrm{KCl}_{2} 2.5 \mathrm{CaCl}_{3}, 1.2 \mathrm{MgSO}_{4}, 1.2 \mathrm{KH}_{2} \mathrm{PO}_{4}, 14.9 \mathrm{HEPES}$ and 5.5 glucose, $\mathrm{pH} 7.4$. The artery was superfused at a tate of $4 \mathrm{ml} / \mathrm{min}$ with physiological salt solution warmed at a temperanure of $37^{\circ} \mathrm{C}$ and continuously gassed with $95 \% \mathrm{O}_{2}$ and $5 \% \mathrm{CO}_{2}$. The pressure at both ends of the artery was monirored using two pressure transducers. Intraluminal flow $(0-100 \mu \mathrm{L} / \mathrm{min})$ could be applied wirhout changes in average pressure.

At the beginning of each experiment, the artery was incubated for 45 minutes at 80 $\mathrm{mmHg}$. The integrity of the endothelium was evaluated by assessing the response to acetylcholine $(1 \mu \mathrm{mol} / \mathrm{L})$ during phenylephrine $(1 \mu \mathrm{mol} / \mathrm{L})$-induced constriction.

Ptessure-induced (myogenic) tone was determined by submitting the arteries to 10 $\mathrm{mmHg}$ pressure steps ranging from 10 to $140 \mathrm{mmH} H \mathrm{H}$ in the absence of intraluminal flow. Pressure was only increased (not decreased or tandomly changed) from 10 to $140 \mathrm{mmHg}$, and augmented to the next level until a stable diameter was teached.

Flow-induced dilatation was studied by increasing the flow rate $(0,4,10,25,50,75$ and $100 \mu \mathrm{l} / \mathrm{min}$ ) at an intraluminal pressure of $80 \mathrm{mmHg}$ (Figure 1). Arteries were 


\section{Monpbologzat Propertien.}

After reactivity measurements, arteries were pressurized at $80 \mathrm{mmHg}$ for one hour in phosphate-buffercd $10 \%$ formaldehyde solution. Fixed vessels were embedded in paraffin, cross sections (4 $\mathrm{Hm}$ ) were stained with Lawson's solution (Boom, Meppel, the Netherlands) to visualize the internal and cxternal elastic laminae. Video images were made from cross sections using a Zeiss axioscope and a standard charge-coupled device camera (Sony). Using J A sofware (Sigma Scan, Jandel Scientific, Corte Madera, CA. USA) the circumference of the internal and external elastic laminae wete determined, and modia cross-sectional area (CSA) was defined as the area enclosed between both laminae. The average number of medial nuclear profiles per cross-section was measuted by counting the mucleat profiles in the smooth muscle layers of hematoxylin-cosine stained cross-scctions at a maginification of $400 \mathrm{X}$ (three cross-sections were counted for each artery and were averaged).

\section{Ditugs.}

$\mathrm{N}^{(1)}$-nitro-L-arginine methyl ester (L-NAME), indomethacin, HOE-140 and sodium mitropruside (SNP) were purchased from Sigma Chemical Co. (St Louis, MO). Other weagents were purchased from Prolabo (Paris, France).

\section{Shativical analysis.}

Results are shown as means $I S E M$. Significance of the differences becween the different groups was determined by ANOVA. Means were compared by paired $t$ test or studentNewman Keuls test. $P<0,05$ was considered to denote statistical significance.

\section{Results}

\section{Geryeral Findings.}

In $T K /$ and $T K+1+$ mice, body weight $(29 \pm 1$ versus $26 \pm 1 \mathrm{~g}$, tespectively) as well as heart weight ( $140 \pm 6$ versus $134 \pm 7 \mathrm{mg}$, respectively) and uterine weight $(0.33 \pm 0.06$ versus $0.33 \pm 0.04 \mathrm{~g}$, respectively) did not differ signiffcantly. During pregnancy, in $T K \%$ and TK +1 mice, body weight ( $46 \pm 2$ versus $43 \pm 2 \mathrm{~g}$, respectively) and uterine weight (12.13 0.77 versus $11.21 \pm 0.98 \mathrm{~g}$, respectively increased to the same extent. Heart weight was significandy increased by day 18 to 19 of pregnancy in $T K /$ mice but not in $T K /+$ mice (155t3 versus $147 \pm 6 \mathrm{mg}$, respectively). When $\mathrm{TK} \%$ mice were compared with

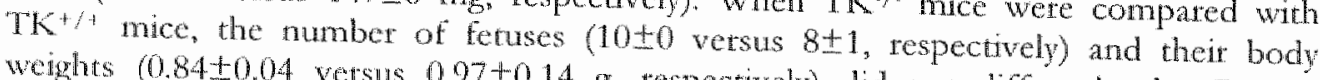
weights $(0.84 \pm 0.04$ versus $0.97 \pm 0.14 \mathrm{~g}$, respectively) did not differ. At day 7 after pregnancy, in $\mathrm{TK}$ and $\mathrm{TK}^{+/ t}$ mice, body weight ( $40 \pm 1$ versus $35 \pm 2 \mathrm{~g}$, respectively) and hert woight ( $200 \pm 5$ versus $164 \pm 9 \mathrm{mg}$, respecrively) remained clevated, but uterine weight (0. $19 \pm 0.02$ versus $0.20 \pm 0.03 \mathrm{~g}$, respectively) was decreased to below the original value.

\section{Arteriat simathe.}

"The stmcture of uterine arteries and mesenteric resistance arteries did not differ between $\mathrm{TK} /$ and $\mathrm{TK} / \mathrm{t}$ mice (Figure 4.2). Neither the arterial lumen diameter, as determined by pressure-diameter curves under passive conditions (Figute 4.3A) nor the media cross 
sectional area and the number of medial cells, detemined on cross sections (Table 4.1), differed between 'TK $/$ and $T K^{+/ t}$ mice.

Pregnancy was accompanied by a regionally selective and statistically significant outward hypettrophic remodeling of the uterine artery (Figure 4.4A). The increases in arterial stuctural diameter and in media ctoss sectional area (Table 4.1) were comparable in $T K /$ and $T K^{+/ t}$ mice.

Table 4.1. Momphometric analysis of uterine and mesenteric arteries of wid-type and rissue katlikrein-deficiont mice.

\begin{tabular}{|c|c|c|c|c|c|c|}
\hline \multirow[b]{2}{*}{ Mensurement } & \multicolumn{2}{|c|}{$\mathrm{NP}$} & \multicolumn{2}{|c|}{ LP } & \multicolumn{2}{|c|}{$P P$} \\
\hline & $\mathrm{TK}$ & TKK- & TK & TK & $\mathrm{TK}^{+1+}$ & $\mathrm{TK}$ \\
\hline \multicolumn{7}{|l|}{ Werine Arteries } \\
\hline Diameter $(\mu m)$ & $335 \pm 9$ & $357 \pm 16$ & $457 \pm 8 *$ & $4,66 \pm 14 *$ & $360 \pm 11$ & $349+16$ \\
\hline $\left.\operatorname{mCSA}(x) 0^{2} \mu m^{3}\right)$ & $4.4 \pm 0.6$ & $4.2 \pm 0.4$ & $6.310 .7 *$ & $6.2 \pm 0.4 *$ & $3.8 \pm 0.2$ & $5.1 \pm 0.5$ \\
\hline $\begin{array}{l}\text { No medial nuclear profiles/ } \\
\text { cross-section }\end{array}$ & $23 \pm 3$ & $25+3$ & $19 \pm 2$ & $19 \pm 1$ & $22 \pm 3$ & $22 \pm 1$ \\
\hline \multicolumn{7}{|l|}{ Mesenteric Resistance Arteries } \\
\hline Diancter $(\mu \mathrm{m})$ & $239 \pm 18$ & $258 \pm 14$ & $271 \pm 13$ & $280 \pm 12$ & $308 \pm 19^{*}$ & $310 \pm 15^{*}$ \\
\hline $\operatorname{mcsA}\left(\mathrm{x} 10^{2} \mu \mathrm{m}^{2}\right)$ & $1.9 \pm 0.4$ & $2.2 \pm 0.4$ & $1.9 \pm 0.2$ & $26 \pm 0.3$ & $2.6 \pm 0.3$ & $2.7 \pm 0.4$ \\
\hline
\end{tabular}

Diameters in the absence of smooth muscle tone obtaned an intraminal prassure of 140 monltg, medit cross-sectional areas (mCSA), and the number of medial nuclen profiles per cross-sccion of paraftinembedded uterine arteries and mesenteric resistance arteries of nonpregnant (NP), late pregnatu (L.P), and

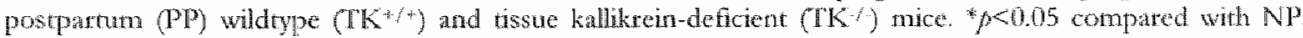
Walues atre ment \pm SEM.
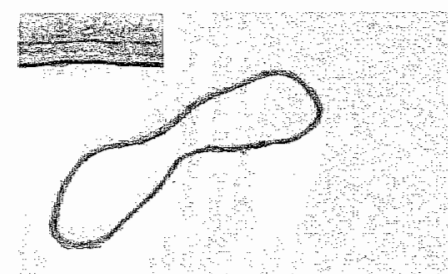

a
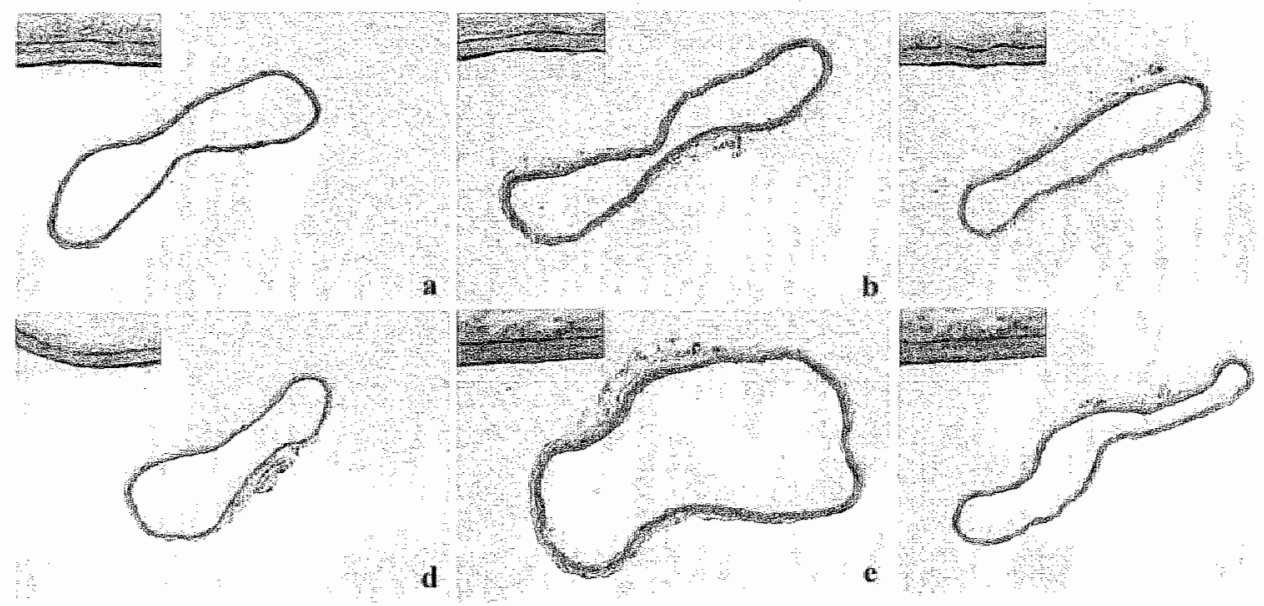

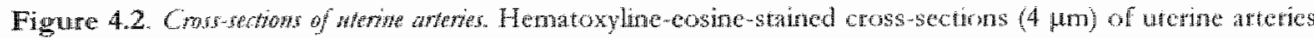

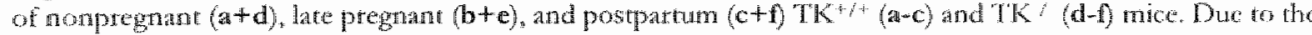
sumall size and mampulation during the process of parafun embedment, cross soctions appear not circalar

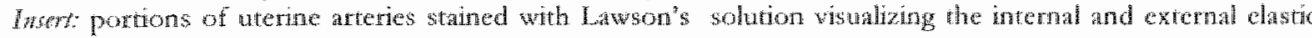
laninace (4 higher magnification). 
By 7 days after pregnancy, most of the stuctural changes in the urenine artery were reversed (Figure 4.5A). In TK mice, the structural lumen diameter and the media cross sectional area did no longer differ from findings before pregnancy (Table 4.1). In IK mice, the structural lumen diameter had regressed to notmal (Figure $4.5 \mathrm{~A}$ ) but a significant media hypettrophy persisted (Table 4.1). The changes in media cross sectional area of the uterine artery during and after pregnancy were not accompanied by significane changes in the number of arterial smooth muscle cells/cross-section in both $\mathrm{TK}$. and TK + mice (Table 4.1). It might be noteworthy that mesenteric resistance arteries, which wete not modified during pregnancy, were enlarged in the postpatum period. This was statistically significant for the lumen diameter in both "TK/- and $T K / / 4$ mice, but the 20 . $30 \%$ media hypertophy did not reach statistical significance in either strain (Table 4.1 ).

A

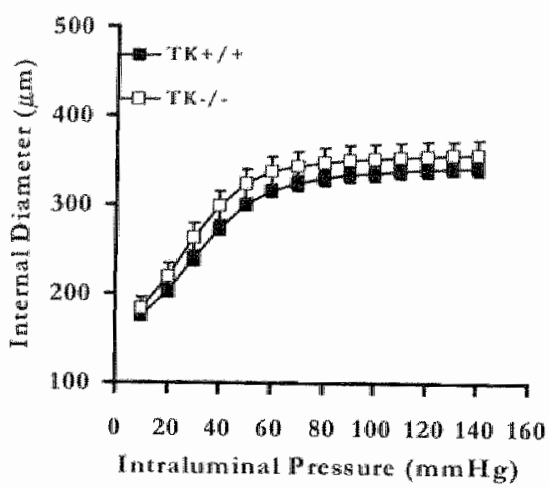

C

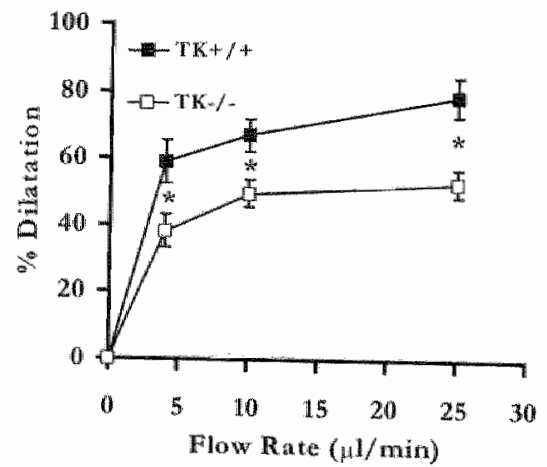

B

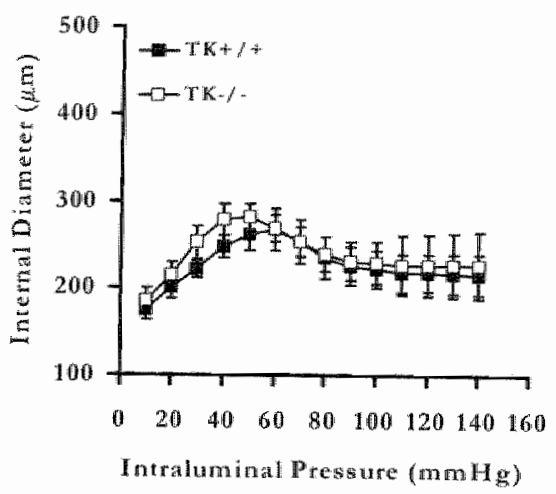

D

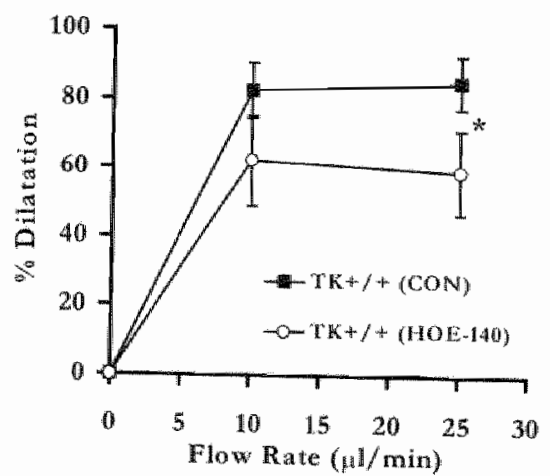

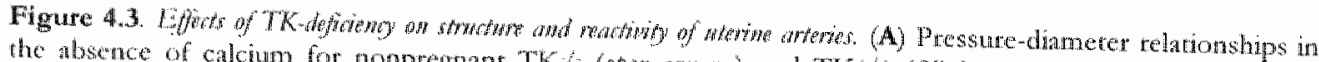

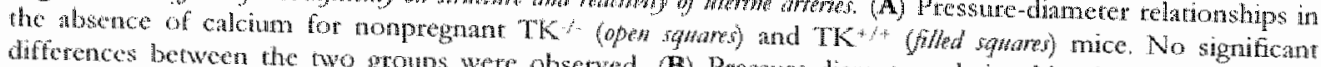
diferences beween the two groups were observed. (B) Pressure-dianeter rationships in the presence of calcim. No defetences in myogenic tone were observed berwean the two strans. (C) Flow-induced diatation

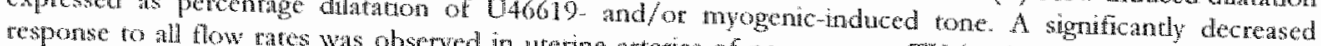
compared to "TK+" (D) Effect of 0.1 umol/ HOF

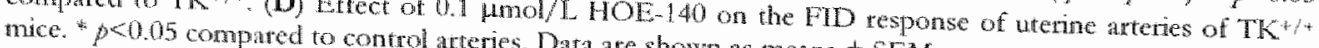




\section{Tanowntritor Reativity.}

Myogenic vasoconstriction (Figure 4.3B) and constriction induced by $1 \mu \mathrm{mol} / \mathrm{L}$ phenylephrine (Table 4.2) did not differ between uterine arteries of $\mathrm{TK} \%$ and $\mathrm{TK}+/$ mice. In the case of myogenic tone, neither the pressure at which the response was induced nor the maximal amplitude (Table 4.2) of the response differed significantly (Figure $4.3 \mathrm{~B}$ ). In uterine arteries of term pregnant mice, when compared to nonpregnant animals, the myogenic constriction at $100 \mathrm{~mm} / \mathrm{Hg}$ and the constriction induced by 1 $\mu m o l / L$ phenylephrine were larger when expressed in absolure values (not shown) but comparable when expressed as precentage reduction of the passive diameter (Figure 4.4 and Table 4.2). This was the case for both $\mathrm{TK}^{7}$ and $\mathrm{TK}^{* /+}$ mice. In uterine atteries of late pregnant $\mathrm{TK}^{* /+}$, unlike $\mathrm{TK} \%$, myogenic constriction was not well maintained at high leve's of transmural pressure (>100 $\mathrm{mmHg}$; Figure 4.4B).

A

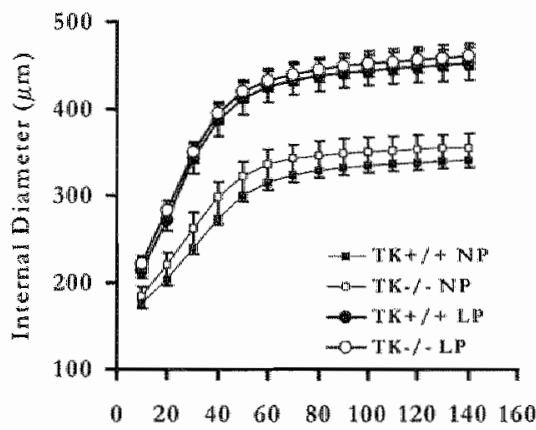

Intraluminal Pressure ( $\mathrm{mm} / \mathrm{Hg})$

C

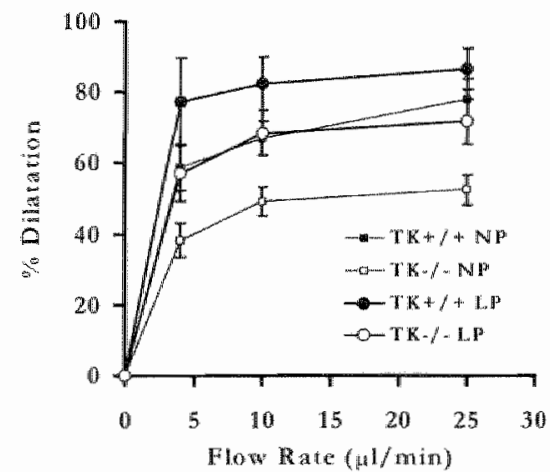

B

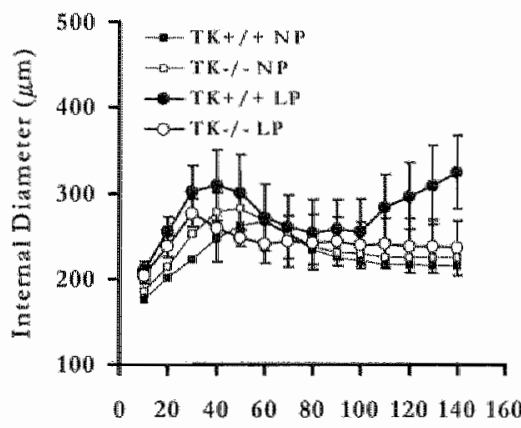

Intruluminal Pressure $(\mathrm{mmHg})$

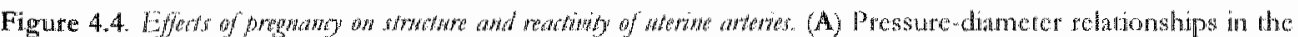

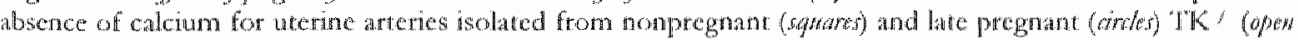

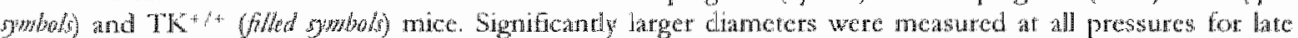
pregrant mice, indicating an ourward, remodeling. No differences were obserwed between the wwo surains. (B) Pressure diameter relationships in the presence of calcium. Myogenic tone measured at $100 \mathrm{~mm} / \mathrm{gg}$ (sec Talbe 2) tended to be higher in uterine arteries of late pregnanat mice. (C) Flow-induced dilatation in uterine arteries of

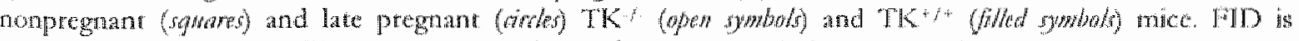
expressed as percentage dilatation of U4661)- and/or mygenic-induced tone. Data are shown ain means \pm SEM. 
A

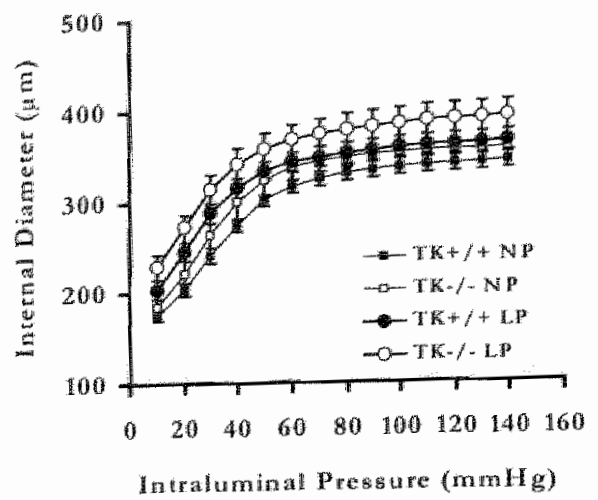

C

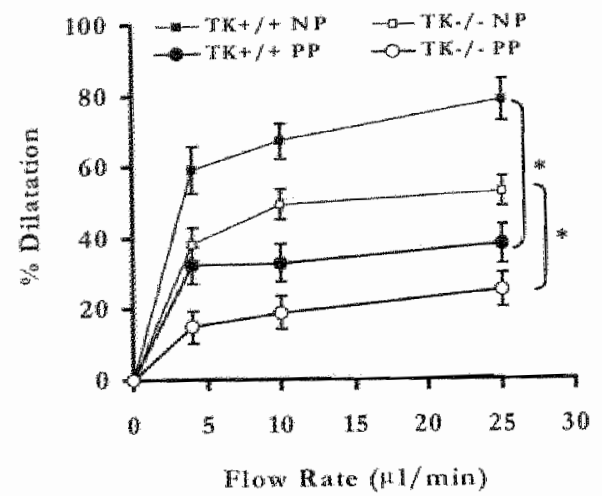

$\mathbf{B}$

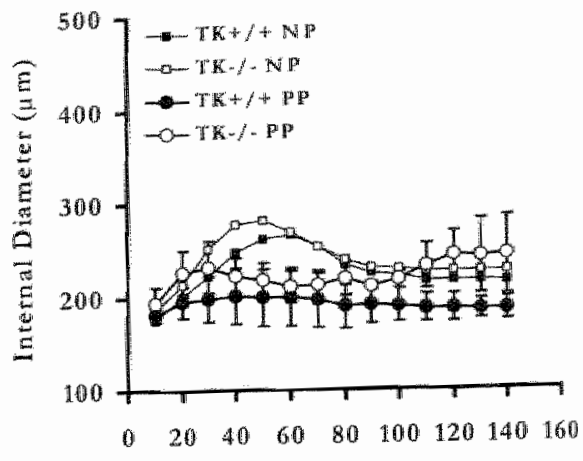

Intraluminal Pressure (mom

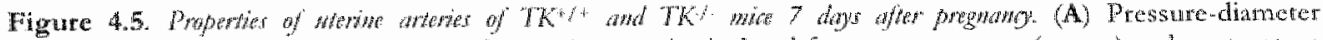

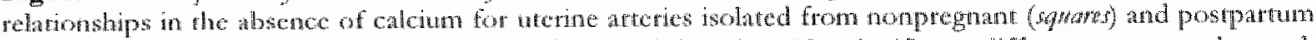

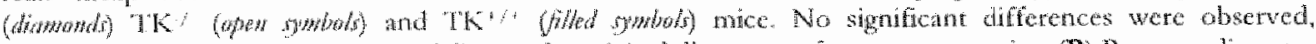

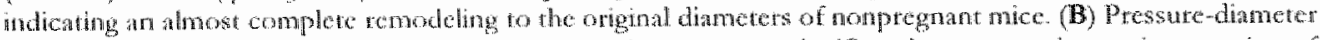

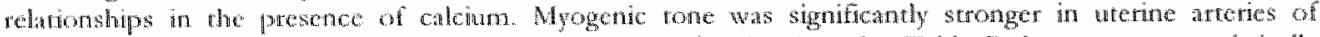

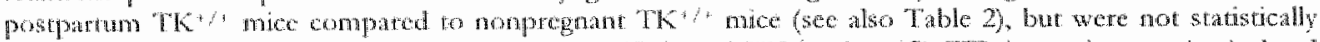

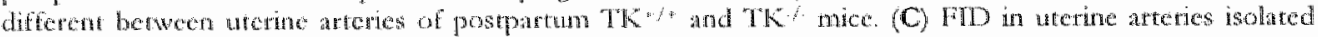

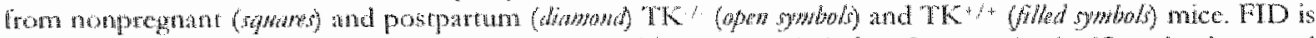

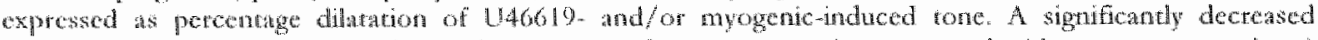

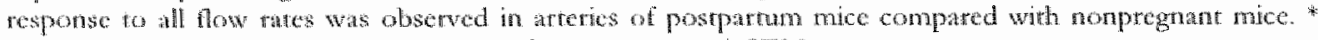

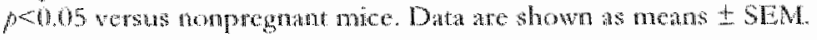

At 7 days after pregnancy, the relative constriction induced by phenylephrine did not differ from observations before and during pregnancy in $\mathrm{TK}^{\%}$ and $\mathrm{TK}^{+/+}$mice (Table 4.2). Sensitivity to myogenic vasoconstriction was increased in the postpartum period when compared to before and during pregnancy (Figure 4.5B). Yet, the amplitude of the myogenic constriction at physiological pressures (80-100 mmHg) was for both TK\% and "TK" comparable to that before pregnancy (Table 4.2). 


\section{Agonist-Indtuced Vasodilatation.}

Dilator responses to $1 \mu \mathrm{mol} / \mathrm{L}$ acetylcholine and intraluminal flow (sce below) were evaluated during $40-45 \%$ constriction induced by a transmural pressure of $80 \mathrm{mmH} H \mathrm{H}$ and 1 to $100 \mathrm{nmol} / \mathrm{L}$ of the tromboxane $\mathrm{A}_{2}$ analogue U46619 (Figure 4.1). This level of preconstriction was comparable for all groups of uterine arteries investigated (Table 4.2). Dilator responses were expressed as a percentage of the response to $100 \mu \mathrm{mol} / \mathrm{L}$ sodium nitroprusside, which induced near maximal dilatation in all cases.

Acerylcholine $(1 \mu \mathrm{mol} / \mathrm{I})$ caused vasodilatation in all uterine arteries studied. The amplitude of this response did not differ between $\mathrm{TK}^{-1}$ and $\mathrm{TK}^{+/+}$mice and was not modified in pregnant and postpartum mice (Table 4.2).

\section{Flon-Induced Vasadilatation.}

Increases in flow rate from 0 to $100 \mu \mathrm{l} /$ min caused flow-dependent increases in diameter in constricted uterine arteries (Figure 4.1). Because the response to $25 \mu \mathrm{l} / \mathrm{min}$ did not differ significandly from responses to higher flow rates, only responses from 0 to 25 $\mathrm{H} / \mathrm{m} / \mathrm{min}$ are depicted in the figures 4.3 to 4.5 .

Flow-induced dilatations were significantly smaller in uterine arteries of 'TK than $\mathrm{TK}^{+/+}$mice at all flow rates tested (Figure $4.3 \mathrm{C}$ ). Maximal responses averaged $55 \pm 4 \%$ $(\mathrm{n}=8)$ and $85 \pm 4 \%(\mathrm{n}=7)$ in $\mathrm{TK} \%$ and $\mathrm{TK}^{+/ 4}$, respectively. In the presence of the $\mathrm{B}_{2-}$ receptor antagonist HOE-140 $(0.1 \mu \mathrm{mol} / \mathrm{L}$; icatibant $)$ flow-induced dilatation of TK $\mathrm{TK}^{+/+}$ arteries was reduced to the level observed in TK $/$ arteries (Figure 4.3D). In the presence of the NO-synthase inhibitor L-NAME $(100 \mu \mathrm{mol} / \mathrm{L})$, flow-induced dilatation was moderately but consistently reduced in uterine arteries of both $\mathrm{TK} /$ and $\mathrm{TK}^{+/+}$mice, and the significant difference between both strain persisted (Table 4.3). In the presence of

Table 4.2. Vasomotor responses of uterine arteries to phenylephrine, acetyloholine, pressute (100 $\mathrm{nm} / \mathrm{g}$ ) and U46619 for wild-type and tissue-kalliketen dehcient mice.

\begin{tabular}{|c|c|c|c|c|c|}
\hline mouse & state & $\begin{array}{l}\text { "o constriction } \\
\text { to } 1 \text { fmol/L Phe }\end{array}$ & $\begin{array}{l}\text { "o dilatation } \\
\text { to } 1 \mu \mathrm{mol} / \mathrm{L} \text { ACh }\end{array}$ & 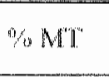 & $\begin{array}{c}\% \text { induced rone } \\
\text { before } 1 \| \mathrm{D}\end{array}$ \\
\hline \multirow[t]{3}{*}{$\mathrm{TK}^{2 / \cdots}$} & $N P$ & $51 \pm 4$ & $85+7$ & $32 \pm b$ & $45 \pm 2$ \\
\hline & $\mathbb{L P}$ & $44 \pm 8$ & $94 \pm 2$ & $43 \pm 7$ & $38 \pm 4$ \\
\hline & PP & $52 \pm 6$ & $87 \pm 3$ & $47 \pm 5 *$ & $45 \pm 4$ \\
\hline \multirow[t]{3}{*}{$\mathrm{TK}$} & $\mathrm{NP}$ & $50 \pm 8$ & $93 \pm 2$ & $35 \pm 5$ & $45 \pm 2$ \\
\hline & LP & $47 \pm 10$ & $87 \pm 5$ & $45 \pm 4$ & $45 \pm 4$ \\
\hline & PP & $51 \pm 4$ & $79+4$ & $30 \pm 7$ & $40 \pm 4$ \\
\hline
\end{tabular}

Abbrevations used: NP: nonpregnant; LP: late pregnant; PP: postpartum; MT: nyogetsic wone; Phe: phengleptheine; ACh: acevicheline. Constrictions of utcrine arteries to 1 fumol/L Pthe are shown as a pereentage of dilatation induced by $100 \mu$ mol/L sodium nitroprussicle. Dilatations of uterine arteries to 1 $\mu_{m} / \mathrm{L} A \mathrm{Ch}$ during $1 \mu \mathrm{mol} / \mathrm{L}$. Phe constriction are shown as percentage of the diancret obaincd at 80 mmHg in the absence of smooth muscte tone and in the presence of $100 \mu \mathrm{M}$ sodium nirryprusside. Percentage MT of uterine arteries was measured at an intraluminal pressure of $100 \mathrm{~mm} / \mathrm{g}$. Constriction before foxw-

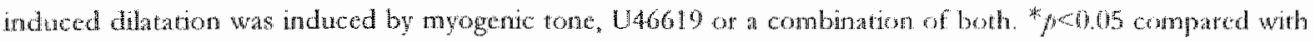
uterine arteries of nonpregnant mice (moan \pm SEM). 
both L-NAME and the cyclooxygenase inhibitor indomethacin (10 $4 \mathrm{~mol} / \mathrm{L})$, flowinduced dilatation was further reduced in artenes of both strains and, again, the significant difference between both strains persisted (Table 4.3). In the presence of both inhibitors, $50 \%$ of the maximal flow-induced dilatation persisted in uterine arteries of both $\mathrm{TK} /$ and TK" mice. The level of constriction was not altered by L-NAME or by the combination of 1. NAME and indomethacin (not shown).

Pregnancy was accomparied by increased flow-induced uterine attery dilatation in both $\mathrm{TK}^{\mathrm{K}} / \mathrm{and} \mathrm{TK} / \mathrm{t}$ mice (Figure 4.4C). Both the sensitivity and the maximal effect were increased. The latter increased from $85 \pm 4 \%$ to $94 \pm 2 \%$ in $\mathrm{TK} / 4$ and from $55 \pm 4 \%$ to $76 \pm 8 \%$ in $\mathrm{TK}$ - micc. During pregnancy, flow-induced dilatation remained significantly smaller in $T^{-1} /$ than $T^{+/+}$. As judged fiom findings with $L$-NAME, the pregnancy relared increase in flow-induced wasodilatation was primarily due to a larger contribution of $\mathrm{NO}$ to the process. In uterine arteries of pregnant $\mathrm{TK}+/+$ mice, L-NAME inhibited $50 \%$ of the flow-induced tesponse while this was only $18 \%$ before pregnancy (Table 4.3 ). Also in TK $\%$ mice the effect of I-NAME was larger during pregnancy (43\% inhibition) than before pregnancy (15\% inhibicion). The L-NAME and indomethacin-resistant part of flow-induced dilatation was not significantly modified by pregnancy. However, in uterine arteries of pregnant mice flow-induced dilatation that can not be attributed to $\mathrm{NO}$ and prostaglandins, did no longer differ significantly between $T K \%$ and $T K \% / 4$ mice (rable 4.3)

While pregnancy was accompanied by increased flow-induced dilatation, this response was reduced to below pre-pregnancy values in the postpartum period (Figute 4.5). Maximal flow-induced dilatation averaged $31 \pm 5 \%$ and $38 \pm 5 \%$ in $T K \%$ and $T K \%$ uterine arteries, respectively (Table 4.3). Although all components seemed to be affected, primatily the L-NAME sensitive part of the flow-induced dilatation was blunted or even abolished. In uterine arteries obtained at 7 days after ptegnancy, differences between TK$\%$ and $T K+4$ mice were no longer statistically significant but this might be due to the small and variable nature of the responses at this time point.

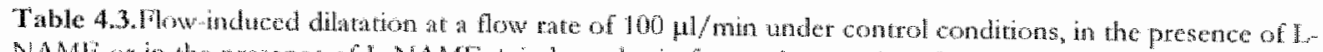

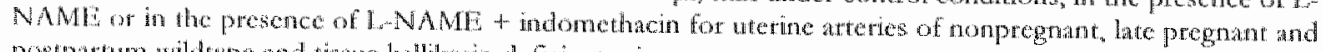
pospratum widlype and tissue kallik tre defichent mice.

\begin{tabular}{|c|c|c|c|c|c|c|}
\hline \multirow[b]{2}{*}{ Combition } & \multicolumn{2}{|c|}{$\mathrm{NP}$} & \multicolumn{2}{|c|}{ L.P } & \multicolumn{2}{|c|}{$\mathrm{PP}$} \\
\hline & "I KK"i; & "IK" & $\mathrm{d} k+$ & $\mathrm{TKK}$ & $\mathrm{TK}$ & $\mathrm{TH}$ \\
\hline Control & $85+5$ & $55+4$ & $94+2+$ & $76 \pm 8+4$ & $38+5=$ & $31 \pm 5+$ \\
\hline INAMI & $70+4 \div$ & $47+7+$ & $48 \pm 6^{\circ}$ & 4446 & $25 \pm 7+$ & $30 \pm 6+$ \\
\hline $\begin{array}{l}\text { MNACI: } \\
\text { mdomethacin }\end{array}$ & $43 \pm 5 \%$ & $27+7+4$ & $34+8 *$ & $36 \pm 6 \%$ & $16 \pm 4 * 4$ & $16 \pm 4 * 1$ \\
\hline
\end{tabular}

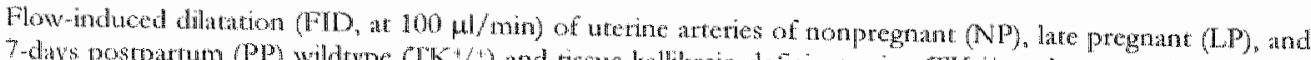

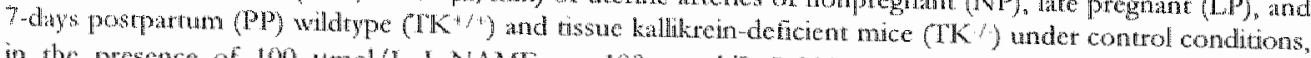

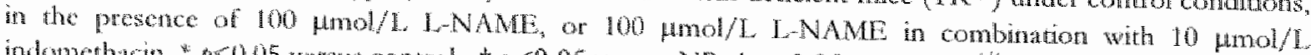

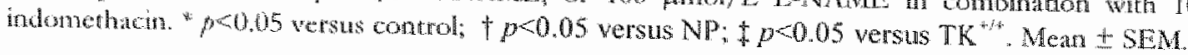




\section{Discussion}

In this study we observed in utetine arteries of the mouse: (1) Hat Row-wand

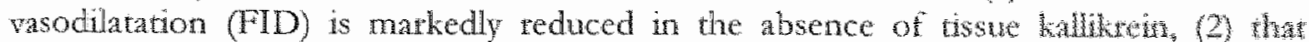
pregnancy is accompanied by rapidy reversible ourward remodeling and upreguhtom of FID, and (3) that these pregnancy-related arterial structural and functional changes are comparable in the absence and presence of TK.

In large conduit arteries a teduced FID was prewiously observed in mice lacking $\mathrm{TK}$ pointing out the importance of locally produced kinins in regulating vasomotor tesponses to shear stress in these vessels. ${ }^{37,35}$ "The objecrives of the present study were 1 to detemine whethet a blunted FID was also present in resistance-sized arteries of TK. mice and 2) whether blunted acute responses to shear stress hamper structural arteriat adaptations in response to chronically altered blood flow. We have chosen pregnancy as a physiological model representing an increased blood flow during gestation and reduced blood flow aftet bith ( 7 days postpartum) in the uterine vascularure of mice. In matny species nomal pregnancy is associated with a matked increase in uterine blood flow. ${ }^{0,35}$ Uterine blood flow has not yet been measured in mice, but cardiac output has been demonstrated to be $64 \%$ higher in late pregnatit mice compared to nonpregnant mice. $5 \% 3$

Uterine arteries of $\mathrm{TK}^{+/ 4}$ and $\mathrm{TK}^{-1}$ mice had similar responses to acetylcholine and sodium nitroprusside, indicating that the release of NO, prostaglandins, and endotheliumderived hyperpolarizing factor $(\mathrm{EDHF})$ by acetylcholine is unaffected by the lack of $\mathrm{TK}$. In this study we have not addressed the direct rasodilatation indwoed by bradykinin on pre-constricted arteries. However, bradykinin has been shown to elicit similat vasoactive responses in $\mathrm{TK}^{+/ *}$ and $\mathrm{TK} /$ carotid arteries, $^{37}$

Uterine arteries of nonpregnant mice responded to intraluminal pressure by developing myogenic tone and to tlow by vasodilatation. Myogenic rone developed at sub-physiological pressures $(50-60 \mathrm{mmHg})$ where no furthet increases in diamerer were recorded and subsequent decreases in diameter were followed at higher pressures until a stable tone was mantained over a wide pressure range. Myogenic tone was similar in arteries of $\mathrm{TK}^{+/+}$and $\mathrm{TK} / \mathrm{mice}$, suggesting no contribution for the kallikrein-kinin system, which is in line writh the endothelium-independent nature of the process. ${ }^{3}$ The exact role of myogenic tone in the uterine vasculature is uncertain. In radial anterics bifurating from the uterine arcuate artery of the at almost no myogenic rone has been observed, but was ptesent during pregnancy. Myogenic responses in the urene circulation might be important in maintaining constant blood flow and in controlling capillary hydrostatic pressute at the site of placentation during pregnancy.

FID was matkedly teduced in uterine atteries of TK/ mice, demonstrating the participation of the kallikrein-kinin system in FID of not only conduit ${ }^{37}$ but also resistance arteries. Aso in systemic resistance arteries such as the mesenteric resistance artery of male mice, FID was found to be reduced (personal obseryatons, S.B.). If at similar reduction in FID occurs in who, one would expect an increase in total peripheral resistance. However, no difference in mean arterial pressure was measured between the two mice strains, 37 possibly as a result of reduced cardiac function in " $\mathrm{K} /$ mice ${ }^{35}$ The contribution of the kalliktein-kinin system was around one-thind of the FID obsetwed in uterine arteries of $\mathrm{TK}^{+/+}$mice. This is in agreement with observations in carotid artertes. 
The difference in response to fow was not due to a diference in myogenic and/or U46619-induced tone before the application of intraminal flow. Kinins, such as bradykimin, act on bradykinin $\mathrm{B}_{2}$ receptors that can stimulate the release of endotheliumderived relaxing factors (EDRFs), $6,89,3 \%, 398 \%$ To demonstrate the involvement of the local kallikrein-kinin system in mediating the FID in uterine arteries, the $B_{2}$ receptor antagonist HOE-140 was used. Since HOE-140 was shown not to affect FID in TK $\%$ carotid arteries, ${ }^{37}$ experiments on uterine arteries of $\mathrm{TK} / \mathrm{m}$ mee were omited. Indeed, FTD was significanty reduced in uterine arteries incubated in the presence of the $\mathrm{B}_{2}$ receptor antagonist, indicating the participation of bradykinin wia $\mathrm{B}_{2}$ receptors in the FID response. FID depended to a large extent on the production of prostaglandins, since the NO synthase blocker L-NAME only matginally suppressed the response to flow, whereas incubation with both L.NAME and indomethacin induced a fifty percent reduction in the response to flow in both strains. There was no significant difference in the relative contribution of $\mathrm{NO}$, $\mathrm{PGI}_{2}$, and the non- $\mathrm{NO}$-non-PGI2 compound(s) to the FID response between $\mathrm{TK}^{+/ t}$ and $\mathrm{TK} /$ arteries. This rules out any compensation by other mediators for the lack of the kalikrein kinin system as judged by these in witm responses, but argues for a role of bradykinin as an autocrine mediator during FID. Compensation by other vasoactive compounds has been observed in eNOS deficient mice. ${ }^{244,524}$ The temaining response to flow might be mediated by EDHF, as was demonstrated in carotid arteries of the mouse. ${ }^{37}$

Ar late pregnancy, heart weight increased in both $\mathrm{TK}^{+/+}$and $\mathrm{TK}^{*}$ mice, which reached statistical significance in the latter mice. Pregnancy results in matked increase in cardiac outpur, due to an increase in stroke volume and heart rate, which might have caused the cardiac hypertrophyn ${ }^{5}$ in 3

At late pregnancy myogenic tone tended to be increased. FID of uterine arteries of both strains of mice was significantly elevated. Predominanty NO was responsible for this upregulation in $\mathrm{FID}$, since the contribution of the L-NAME-sensitive compound was significantly increased in both $\mathrm{TK}^{+/}$and $\mathrm{TK}^{\%}$ mice. Because $\mathrm{NO}$ has been reported to inhibit the production of other endothelium-derived factors, ${ }^{53}$ the observed upregulation of the role of $\mathrm{NO}$ in FDD cluring pregnancy might be accompanied with a drop in the relative importance of prostaglandin and non-prostaglandin/non-NO mediators. Pregnancy increases uterine attery NO-nediated vasodilatation, via increased basal NO production (NOS) in rats," guinea pigs, ${ }^{581}$ ewes, 36 and humans, ${ }^{39}$ Expression of eNOS mRNA and eNOS protein is increased in ovine pregnancy, 37 which likely follows

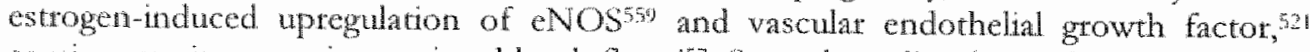
causing an increase in uterine blood flow. ${ }^{5}$ ? Several studies have demonstrated an increased $\mathrm{F}$ ID of arteries experiencing a rise in blood flow. For instance, exercise raining augmented $\mathrm{CID}$ in tat skeletal muscle arterioles. ${ }^{488}$ The augmented dilator response was due to increased release of NO and prostaglandins. More recently, Loufrani and colleagues used a surgical model to apply an increased blood flow in mesenteric tesistance arteries and observed a greater FID and eNOS expression in these flow loaded arteries. 32 In view of out similar hudings in $\mathrm{TK}^{+/}$and $\mathrm{TK} /$ mice, $\mathrm{TK}$ does not seem to be required for this chronic upregulation.

During pregnancy, blood flow through the uterine circulation increases substantially. 160,335 Due to this increase in wall shear stress the uterine artery undergoes a 
structural increase in diameter, termed outwat remodaling. A limitation of the present study is the absence of a quantitative uterine blood flow measurement. However, we have reasons to speculate that blood flow increases substantially in late pregnant mice. Firsty, uterine blood flow has been shown to increase dramatically ( 140 - fold) at tem in tats.6.3 Secondly, cardiac output increases $64 \%$ during murine pregnancy ${ }^{50}$ Because uterus weight and fetal weight and number are not statistically different between $\mathrm{TK}{ }^{\mathrm{t}+}$ and $\mathrm{TK}$ Tlate pregnant mice, we believe that the uterine blood flow rise is comparable in both strains. However, the presence of a reduced acute FID in TK/ uterine arrenes may indicate a somewhat higher wall shear stress. Since wall shear stress is the primary stimulus to initiate flow-induced arterial remodeling, we questioned whether a blunted FID would result in an altered remodeling nesponse during and after pregnancy.

In previous studies, chronic elevations and reductions in blood flow have been reported to respectively induce outward and inward atterial remodeling, $3,3,32,46,4,44,47,547,530$ This adaptive response allows a nomalization of wall shear stress and is accompanied by changes in arterial smooth muscle cell number, size and phenotype in order to restore circumferential wall stress. 7,555 Here we confirm that chronic increases in blood flow, as they occur during pregnancy, lead to an outward hypertrophic temodeling of utrine arteries. The increase in arterial wall mass was due ro vascular smooth muscle cell hypertrophy tather than hyperplasia, since no difference in the number of medial smooth muscle cells were observed. The outward remodeling and increase in media crosssectional area were similar in $T^{+/ t}$ and $T K /$ mice, despite a significandy different acute response to flow before and during pregnancy. This indicates that 'TK deficiency and blunted FID do not compromise stuctural and funcrional atterial changes in response to long-term blood flow elevations. A similar conclusion was drawn tecently in a study using desmin knock-out mice. Desmin is a constirutive subunit of the intemediate filaments in smooth ruuscle cells. Although a blunted FID of mesenteric resistance arteries was measured in these mice, the outward hypertrophic remodeling in response to a 4-week period of a doubling of blood flow was even higher compared to wild-type mice. ${ }^{327}$ Vimentin, another intermediate filament of the cytoskelleton, is involved in the transduction of mechanical strain, and shear stress. We previously showed an excessive luminal enlargement of carotid arteries when exposed to a chronic increase in blood flow,

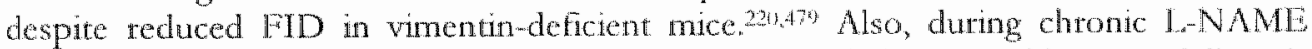
treament, which blunts FID, flow-induced ourwat hypertrophic remodeling is maintained in the rat. ${ }^{84}$ All together, these observations suggest a dissociation between acute and chronic arterial responses to wall shear stress.

$T K$ and bradykinin $B_{2}$ receptor $m R N A$ and protein are expressed in several ussues of the utetus and myometrical blood vessels during human pregnancy "However, lack of "TK does not cause any noticeable complications in murine pregnancy, as fat as uterine arterial remodeling, fetus weight and the number of pups delivered are considered. More subtle consequences can, however, not be excluded. To this and, cardiovasculat and tenal function will have to be critically compared in the heterozygous offspring of $\mathrm{TK}$ and $\mathrm{TK}^{+}+\mathrm{t}$ mothers.

Body weight remained elevated in postpartum mice, possibly because of increased food intake to facilitate lactation. Heart weight also remained increased compared to heart weights of nonpregnant mice. The cardiac hypertrophy most likely resulted from the 
increased cardiac output and heart tate at late pregnancy and at 3 days postpartum as measured by Wong and colleagues. ${ }^{3 \% 3}$ Myogenic tone was stronger in uterine arteries of 7 days postpartum mice compared to virgin mice. Remarkably, the FID response was markedly reduced in uterine artenes 7 days postpartum, suggesting a reduced shear stress sensing of the endothelium, despite nomal acetylcholine-mediated vasorelaxation. We have currenty no explanation for this phenomenon. An increased myogenic tone and a decteased FJD lead to an increased contractility, possibly reducing the risk of excessive blood loss after delivery.

As observed from the passive pressure-diameter curves of uterine arteries of postpartum mice, diamerets no longer differ from their nonpregnant values. Media crosssectional area of uterine arteries of 7 days postpartum mice were similar to nonpregnant walues, reflecting an inward hypotrophic remodeling compared to uterine arteries of mice neat tem. Agan, the bunted acute responses to flow are not sufficient to disturb structural arterial adaptations in response to long-term changes in blood flow.

A surprising finding was the outward remodeling observed in mesenteric resistance arteties of 7 days postpartum mice. Feeding of the pups requires a higher food intake than normal, likely to inctease blood flow through the mesentery, leading to this inctease in diameter. In rats with streptozotocin-induced diabetes, blood flow to the small intestine is markedly increased due to marked hyperphagia. ${ }^{225}$ Our group previously showed that this rise in mesenteric blood flow resulted in a larger diameter of the mesenteric resistance artery of diabecic rats compared to control tats. win?

In conclusion, we confirmed the role of the kallikrein kinin system in acute FID via $B_{2}$ teceptors at the level of tesistance arteries. Absence of this kalliktein-kinin system seems not to be compensated by other mediators, that can mediate FID in TK arteries. Furthemote, we demonstrated the dynamic nature of physiological arterial temodeling and atteriogenesis during and after pregnancy, and show that TK deficiency and blunted FID do not compromise arterial structural and functional changes during and after pregnancy, which are accompanied by marked changes in local blood flow. These obserwations point to a dissociation between acute and chronic arterial responses to wall

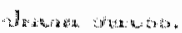


Chapter 5

\section{Intra-Arterial Bradykinin and Vasodilatation in Relation to Acute and Chronic Changes in Blood Flow}

Rob H.P. Hilgers, Paul M.H. Schiffers, Jo G.R. De Mey.

Department of Phamacology \& Toxicology. Cardiovascular Research Instirute Mastricht (CARTM), Universiteit Maastricht, the Netherlands. 


\section{Abstract}

Objective. Investigate the rolle of endogenous intra-atterial bradykinin in chronic modulation of endothelium-dependent vascular reactivity.

Methods and Reswls. We investigated the vasodilator tesponse to flow (shear stress) and acetylcholine ( $\mathrm{ACh}$ ) in pre-constricted isolated uterine arteries of nonpregnant, late pregnant and 7-days postpartum tissue angiotensin-converting enzyme-deficient ( $t$ $\mathrm{ACE} / \mathrm{)}$ ) and wid type ( $\mathrm{t}-\mathrm{ACE}+\%$ ) mice. Vasodilatation was measured in the absence and presence of either the bradylanin $B_{2}$-receptor antagonist $\mathrm{HOE} 140$, the nitric oxide synthase inhibitor: $\mathrm{N}^{\text {(wi }}$-ntro-J-arginine (NIA) or a combination of both. Under control situations, $\mathrm{ACh}$ and shear stress induced similat relaxations in arteries of $\mathrm{C}-\mathrm{ACE}+\mathrm{C}^{+}$and $\mathrm{t}$ ACE $\%$ mice. HOE 140 reduced the responses to $A C h$ and shear stress to a larger extent in uterine arteres of $t-A C E^{/ /}$mice compared to $t-A C E^{+/+}$mice. NLA reduced these responses to the same extent in both strains. No changes in the responses no ACh were observed during and after pregnancy in both strains. The inhibitory action of NIA on shear stress-induced rasodilatation was more substantial in urenine arteries of latepregnant $\mathrm{C}-\mathrm{ACE}+/+$ mice compared to $\mathrm{t}-\mathrm{ACF} / \mathrm{-mice}$, suggesting enhanced telease of nimic oxide in late pregnant $t-A C E^{-/}$mice. The role of bradylinin in $A C h$ and shear stressinduced vasodilatations was more pronounced in uterine arteries of late pregnant $\mathrm{t}-\mathrm{ACE} /$. mice. By 7 days after pregnancy, shear stress-induced vasodilatation was markedly blunted in uterine arteries of both mouse strains.

Conchusion. These observations confirm (1) the release of vasodilator concentrations of bradykinin by chemical and mechanical endothelium-dependent stimuli, and (2) the modulatory role of bradykinin during flow-minduced dilatation of uterine arteries in sinuations of chronically altered uterine blood flow. 


\section{Introduction}

The endothelium is a main determinant of vasomotor tone in vivo, ${ }^{4}$.no Endotheliumdependent vasodilatation can be triggered by both chemical and mechanical stimuli. Endothelium-dependent agonists include bradykinin, which in turn can be released by the endothelium in response to an increase in wall shear stress. ${ }^{37,223}$ 'The physiological significance of endogenous bradykinin in endothelium-dependent vasodilatation largely remains to be established.

Endothelium-dependent vascular reactiviry is susceptible to chronic modulation. Various cardiovascular diseases are associated with endothelial dysfunction. ${ }^{531}$ In several cases this can be reversed by ACE inhibition and angiotensin type 1 ( $A T^{\prime}$ ) receptor antagonists. ${ }^{36,4+4,481}$ On the other hand, chronic increases in local blood flow and estrogens have been found to enhance endothelium-dependent vasodilatation through an upregulation of endothelial nitric oxide synthase (eNOS). The latter processes contribute to the dramatic increase in uterine blood flow during pregnancy. The role of bradykinin in chronic modulation of endothelium-dependent wasculat teactivity has not been elaborated in great detail.

We evaluated the contribution of endogenous bradykinin to acute and chronic modulation of agonist- and flow-induced atterial wasodilatation. To this end we studied uterine arteries before, during, and after pregnancy. W/e used both wild-type mice and mice deficient in tissue angiotensin-converting enzyme ( $t-A C E)$, which degrades bradykinin. Responses of isolated arteries were recorded in the absence and presence of the bradykinin $\mathrm{B}_{2}$ receptor antagonist HOE 140 and the NOS inhibitor $\mathrm{N}^{\text {(t) }}$-nitro-Larginine.

\section{Methods}

\section{Aminals}

The generation of mice with C57Bl6/SV generic background and lacking tissuc-bound angiotensin converting enzyme ( $\left(\mathrm{ACE} / \mathrm{)}\right.$ ) has been described by Esther and colleagues ${ }^{1+1}$ Nice heterozygous for the mutated $\mathrm{ACE}$ allele $(\mathrm{t}-\mathrm{ACE}+\mathrm{s})$ were mated to obtain mice homozygous for the mutated allele ( $\mathrm{t}-\mathrm{ACE} / \mathrm{)}$ ) and their wild-type littermates $(\mathrm{t}-\mathrm{ACE}+\mathrm{A}+\mathrm{t})$. All animals were housed in groups of 2 to 4 and had free access to standard food (SRMA1210; Hope Farms, Woerden, the Netherlands) and tap water. Adult fermale mice (age 4-5 months) were used to study their vasculature; comparing $\mathrm{t}-\mathrm{ACE}$ \% with $\mathrm{t}-\mathrm{ACE}$ / . In view of the infertility of male $\mathrm{t}-\mathrm{ACE} / \mathrm{mice}$, male $\mathrm{t}-\mathrm{ACE} / 4$ mice were used for mating vingin (in the remainder of this article referred as nonpregnant) $\mathrm{t}-\mathrm{ACE}+/+$ and $\mathrm{t}-\mathrm{ACE} / \mathrm{micc}$. All experiments were conducted according to institutional guidelines. Experiments were performed in nonpregnant, late pregnant (day 18 of furst pregnancy), and postpartum (7 days after first delivery).

\section{Preparation of Blood Vessels and Experimental Set-Up}

Afrer euthanasia (overdose of pentobarbital), the uterus was dissected and pinned out on a petridish (coated with a layer of silicon) filled with modified Krebs-Ringer buffer 
(composition in mmol/s: $144 \mathrm{NaCl}_{3} 4.7 \mathrm{KCl}, 2.5 \mathrm{CaCl}_{2}, 1.2 \mathrm{MgSO}_{4}, 1.2 \mathrm{KH}_{2} \mathrm{PO}_{4,} 14.9$ HEPES, and 5.5 glucose, pH 7.4). Adipose-and connective tisswe were carefully removed from the atterics. From every mouse a segment $(3 \mathrm{~mm}$ ) of the main uterine artery at midpoint of the uterine arcade was isolated. The artery was mounted in an arteriograph system (Ling System Instrumentation, Burlington, VT, USA) as previously described. ${ }^{23}$

Ar the beginning of each experiment, the artery was incubated for 45 minutes at 80 minH. "The integrity of the endothelium was evaluated by assessing the response to accrylcholine $(1 \mu \mathrm{mol} / \mathrm{L})$ during phenylephrine $(1 \mu \mathrm{mol} / \mathrm{L})$-induced constriction. Arteries that dilated $<60 \%$ of passive diameter (see below) were excluded.

Passive lumen diameter at $80 \mathrm{mmHg}$ and medial cross-sectional area of fixed uterine arteries were detemined as described previously. 223 Flow-induced dilatation (FID) was studied by applying an intraluminal flow resulting in a wall shear stress of $5 \mathrm{dyne} / \mathrm{cm}^{2}$ at an intraluminal ptessure of $80 \mathrm{mmHg}$. Arteries wete constricted either spontaneously via myogentc tone or in combination with the tromboxane analogue U46619 to a level of approximately $50-60 \%$ of the passive diameter. Flow-induced dilatation was calculated as followis:

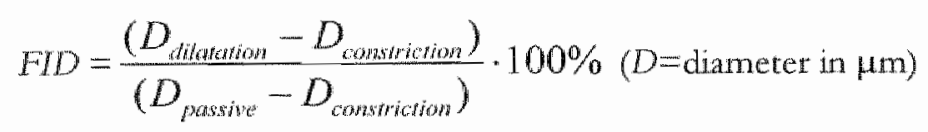

At the end of the experiment, intraluminal flow was stopped and a constriction returned. Agonist-induced endothelinm-dependent vasodilator tesponses were evaluated by constructing a cumulative concentration-response curve (CRC) for acetylcholine (ACh, $0.01-10 \mu \mathrm{M})$. Sensitivity $\left(\mathrm{pD}_{2}=-\log \left[\mathrm{EC}_{50}\right)\right.$ and the maximal response to the agonist (Emax) were detemined by sigmoidal curve fitting of individual curves (Graphpad Prism ${ }^{\text {TM }}$ 2.01. software).

FID was tested in the presence of either the $\mathrm{NO}$ synthase inhibitor $\mathrm{N}^{\text {to }}$-ritro Larginine (NLA, $30 \mu \mathrm{mol} / \mathrm{L})$, the bradykinin $\mathrm{B}_{2}$ receptor antagonist HOE $140 \quad 0.1$ Hmol/L), or the combination of both dugs. For each WID experiment a different uterine artery segment was used.

Drogs

U46619 was purchased from Calbiochem (Damstadt, Germany). Acerylcholine was puchased from Serva Finbiochemica (Heidelberg, Germany). L-phenylepheine, norpinephrine, No nitro-L-arginine, sodium nitroprusside and HOE 140 were purchased From Sigma Chemical (St Lous, USA).

Statishat Analysis.

Results ate shown as means 1 SEM. Significance of the differences berween the different groups was determined by ANOVA. Means were compared by paired t rest or studentNewman Keuls test. $P<0.05$ was considered to denote statistical signiffcance. 


\section{Results}

\section{Uterine Artery Strwhure}

Uterine artery passive diameter at an intraluminal pressure of $80 \mathrm{mmHg}$ is shown in Table 5.1. As observed previously, ${ }^{224}$ passive lumen diameters of uterine arteries of nonpregnant t-ACE mice are significantly wider compared to their wild-type counterparts. Pregtancy resulted in a significant outward remodeling of the utetine arteties in both strains, confirming our previous studies. 223,22 This increase in internal diameter tended to be smaller in $t-\mathrm{ACE}^{-/-}$mice compared to $\mathrm{t}-\mathrm{ACE}+/ \mathrm{mice}(\triangle 96 \mu \mathrm{m}$ in $\mathrm{t}-\mathrm{ACE} / \mathrm{-versus} \triangle 129$ $\mu \mathrm{m}$ in $\mathrm{t}-\mathrm{ACE}+\dagger)$. Medial cross-sectional area of uterine atteries increased to the same extent in $\mathrm{t}-\mathrm{ACE}+\mathrm{H}$ (from $2.5 \pm 0.3$ to $5.5 \pm 0.6 \times 10^{3} \mathrm{\mu m}^{2}$ ) and $\mathrm{LACE}$ (from $2.9 \pm 0.3$ to $5.4 \pm 0.3 \times 10^{3} \mathrm{Hm}^{2}$ ) mice. At 7 days postpartum, uterine attery structure had regressed to pre-pregnant values in both stratios (Table 5.1).

Table 5.1. Lumen dimeter (LD) and constrictor responses to phenylephtine (Phe) in uterine arteries of nompreganat (NP), late- pregnant (LP) and 7 days postpartum (PP) wald-type (twClats) and rissue

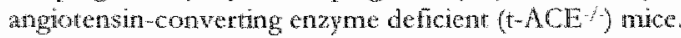

\begin{tabular}{|c|c|c|c|c|c|}
\hline strain & statice & $\begin{array}{l}\text { Passive LD at } \\
80 \mathrm{mmHg}(\mu \mathrm{m})\end{array}$ & $\begin{array}{c}\text { LD in the } \\
\text { presence of } \\
1 \text { Hmol/L Phe }\end{array}$ & $\begin{array}{c}\text { constrictor } \\
\text { response to } \\
1 \mu \mathrm{mol} / \mathrm{L} \text { Phe } \\
(\mu \mathrm{m})\end{array}$ & $\begin{array}{l}\text { constrictor } \\
\text { response to } \\
1 \mu \text { mol } / \mathrm{L} \text { Phe } \\
(\%)\end{array}$ \\
\hline \multirow[t]{3}{*}{$\mathrm{t} \sim \mathrm{ACE}^{+/ 4}$} & $\mathrm{NP}$ & $264 \pm 5$ & $110 \pm 6$ & $154 \pm 6$ & $58 \pm 2$ \\
\hline & LP & $393 \pm 10 *$ & $156 \pm 10 *$ & $237 \pm 10^{*}$ & $60 \pm 2$ \\
\hline & $\mathrm{Pp}$ & $300 \pm 29$ & $138 \pm 25$ & $162 \pm 27$ & $55 \pm 7$ \\
\hline \multirow[t]{3}{*}{$\mathrm{t}-\mathrm{ACE}$} & NP & $284 \pm 10 t$ & $125 \pm 8$ & $159 \pm 8$ & $56 \pm 2$ \\
\hline & $\mathrm{LP}$ & $380 \pm 17 *$ & $171 \pm 10 *$ & $209 \pm 13 *$ & $55 \pm 3$ \\
\hline & $\mathrm{PP}$ & $279 \pm 33$ & $179 \pm 20$ & $100 \pm 20$ & $45 \pm 5$ \\
\hline
\end{tabular}

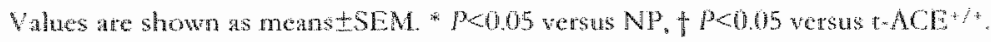

\section{Uterine Artery Tanowstrition}

Phenylephrine (1 Hmal/L) induced comparable constrictions in uterine arteries of $\mathrm{ACE}$. 7 and $[-A C E+/ 4$ mice (Table 5.1). Absolute vasoconstriction ( $\mu \mathrm{m}$ ) in response to phenylephrine $(1 \mu \mathrm{mol} / \mathrm{L})$ was larger in uterine arteries of late pregnant mice compared to nompregnant and postpartum mice, but relave vascoconstriction (\%) was not modified by pregnancy (Table 5.1).

\section{Dilator Restonser to Acetyoline}

Responses to $\mathrm{ACh}\left(\mathrm{pD}\right.$ and $E_{\text {max }}$ ) did not differ between uterine arteries of nompregnant t-ACE- and $t-A C E^{+/ 4}$ mice $(6.63 \pm 0.09$ and $96 \pm 1 \%$ versus $6.78 \pm 0.09$ and $96 \pm 1 \%$, respectively; Table 5.2). The bradykinin $B_{2}$ receptor antagonist HOE-140 (0.1 $\left.\mathrm{mmol} / \mathrm{L}\right)$ caused a rightward shift of the concentration-response curve and a significant reduction in the maximal vasodilatation (Ema) in uterine arteries of $-A C E-$ mice (Figure 5.1A). This 
latter effect was significantly latger in $t-A C E$ mice $\left(\triangle E_{\text {max }} 14 \%\right.$ ) than in $\mathrm{t}-\mathrm{CE}$ - mice $\left(\Delta E_{\text {max }}=5 \%\right.$, Table 5.2). The nitric oxide synthase inhibitor nitro-L-arginine (NLA) reduced the responses to a larget extent by causing a significant blunting of the maximal response to $\mathrm{ACh}$ in uterine arteries of both strains (Table 5.2). The combination of $\mathrm{HOE}$ 140 and NA $A$ had no additional inhibitory effect on the ACh-induced vasodilatation in urerine arteries of nompregtant mice (Table 5,2).

The sensitivity and amplitude of ACh-induced wasodilatarions were similat in uterine arteries of larepregnant mice of both strains, and did not differ in values from nonpregnant mice (Figure 5.2A and Table 5.2). However, HOF 140 had a marked inbibitory effect on the maximal response and sensitivity to $A C h$ in uterine arteries of latepregnant $\mathrm{t}-\mathrm{ACE} / \mathrm{mice}$ compared to late-pregnant $\mathrm{ACE}+\mathrm{H}$ mice (Figure 5.2A, Table 5.2). NLA had comparable inhibitory effect in uterine arteries of $t-A C E^{+/ *}$ and $-A C E$. mice (Mable 5.2). However, the presence of both HOE 140 and NLA seemed to have an additive inhibitory effect on the vasodilator responses to $\mathrm{ACh}$ in pregnant mice of both strans (Table 5.2 ).

Stmilar to nonpregnant and late-pregnant mice, the sensitivity and amplitude of ACh-induced vasodilatations did not differ in uterine arteries of 7-days postpartum mice (Figure 5.3A and Table 5.1). Again, HOE 140 had a marked effect on both the maximal response and sensirivity to $\mathrm{ACh}$ in uterne arteries of $\mathrm{t}-\mathrm{ACE}-\mathrm{t}$ mice, whereas it had almost no inhibitory effect in uterine arteries of $\mathrm{t}-\mathrm{ACE}+$ mice (Figute 5.3A, Table 5.2). NLA reduced the tesponses to $A C h$ to the same extent as observed in uterine arteries of nonpregnant mice (Table 5.2).

\section{Dilator Responses to Sbear Stress}

Application of a shear stress stimulus (5 dyne/ $\left.\mathrm{cm}^{3}\right)$ resulted in a dilatation, which tended to be larger in uterine arterics of $\mathrm{t}-\mathrm{ACE} /$ mice (Figure 5.1B). HOE 140 reduced this shear stress-induced or flow-induced diatation (F-1D) to a greater extent in uterine arteries of $-A C E$, $(\Delta 42 \%)$ than $t-A C E+/ 329 \%$ mice (Figure $5.1 B)$. NLA reduced FID to the

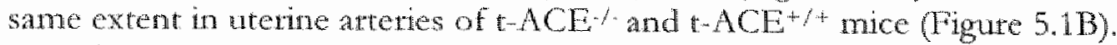

At term pregnancy the ratave amplitude of FID was comparable in utene arteries of both strains and comparable to that of nonpregnant mice (Figure 5.2B). The contribution of bradykinin to $\mathrm{CD}$ was significantly larger in UAs of $\mathrm{ACE} / \mathrm{As}$ shown by the greater inhibitory effect of $40 \mathrm{C}$ 140 (Figute 5.2B). NL A had a far greater inhibitory effect on $\mathrm{HD}$ in uterine arteries of $\mathrm{t}-\mathrm{ACE} / \mathrm{t}$ mice compared to $\mathrm{t}-\mathrm{ACE}$ - mice (figure 5.2B).

FID in uterine arteries of postpartum mice was markedly reduced when compared to observations before and after pregnancy. Moreover, neither $\mathbb{B}_{2}$ receptor antagonism nor NOS inhbition reduced the $\mathrm{TDO}$ in uteine artertes of $t-A C E \%$ and $t-A C E+$ mice at 7 days after pregnancy (Figure 5.3B). 
A

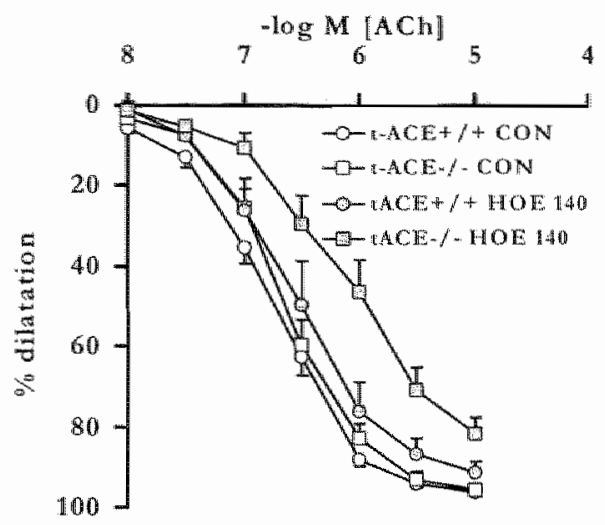

B

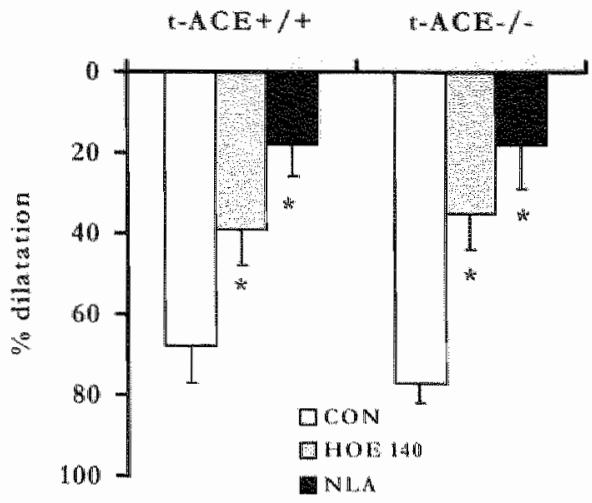

Figure 5.1. Cumulutative concentration-tesponse curves of aceflcholine in urerine arteries under control conditions (CON; open symbols), and in the presence of $0.1 \mu \mathrm{mol} / \mathrm{L}$ of the bradykinin $\mathrm{B}$-receptor anagonist HOE 140 (shaded symbols) from nompregnant $\mathrm{ACE}$; (circles) and $\mathrm{ACE}$ (squares) nice for charty responses in the presence of the nitric oxide synthase blocker $\mathrm{N}$-nitro-1.arginine were omitted (sec fable 5.2) (A). Dilatation of uterine atreries in response to shear stress ( $5 \mathrm{dyne} / \mathrm{cm}^{3}$ ) under control conditions (open bars),

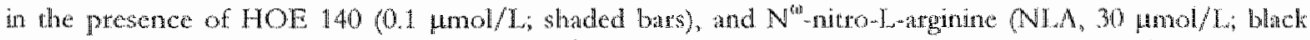

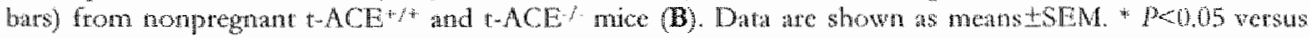
CON.

A

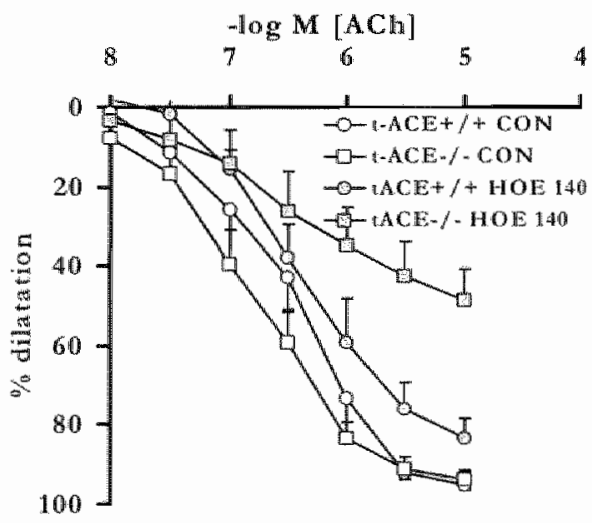

B

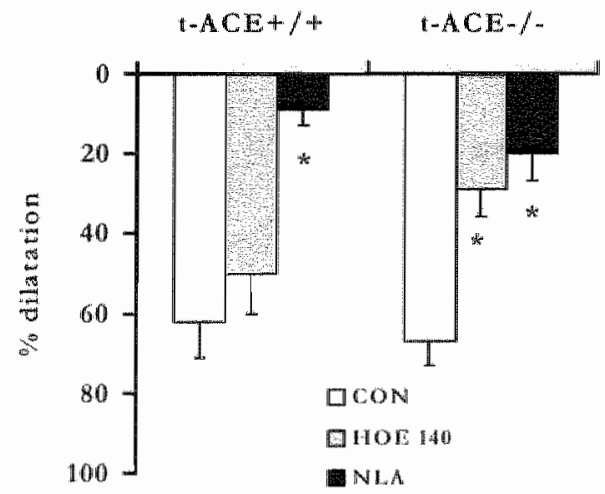

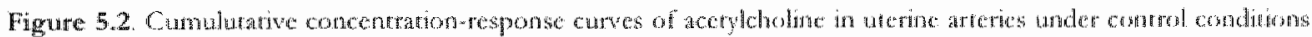

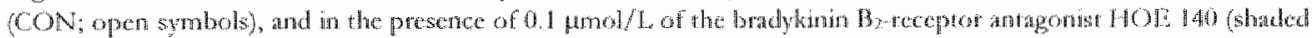
syzubolsy from late pregrand $\mathrm{t}-\mathrm{ACE} / \mathrm{A}$ (circles) and $\mathrm{t}-\mathrm{ACC}$ (squares) mice. For clatity wesponases in the presence of the ninche oxide synthase blocker $N^{\text {th }}$-ratro-Larginine were omitted $(A)$. Dilatation of uxerine arteries in response to

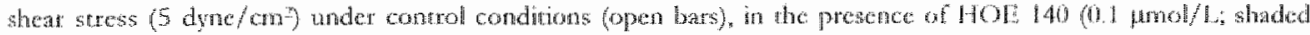

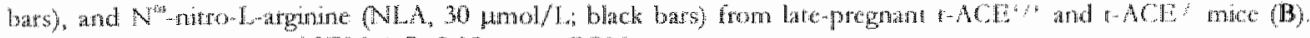
Data are shown as means asEM. * $P<0.05$ wersus CON. 


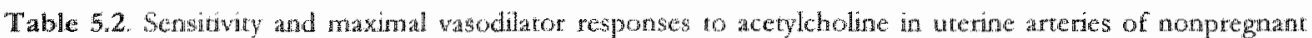

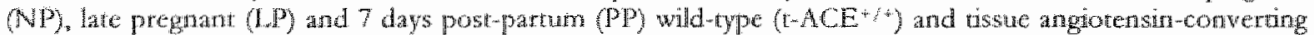
chanme deficiont ( $\mathrm{ACE}$ ) mice.

\begin{tabular}{|c|c|c|c|c|c|c|c|}
\hline \multirow[b]{2}{*}{ Condition } & \multirow[b]{2}{*}{ parameter } & \multicolumn{3}{|c|}{$\mathrm{t}-\mathrm{ACE}$} & \multicolumn{3}{|c|}{$1-\mathrm{ACE}^{/ /-}$} \\
\hline & & NP & $L P$ & PP & NP & $\mathbf{L P}$ & $\mathrm{PP}$ \\
\hline \multirow{2}{*}{ Control } & $\mathrm{WD}_{2}$ & $6.78 \pm 0.09$ & $6.47 \pm 0.14$ & $7.07+0.09$ & $6.63 \pm 0.09$ & $6.78 \pm 0.16$ & $6.71 \pm 0.08$ \\
\hline & $\mathrm{E}_{\text {manz }}$ & $96 \pm 1$ & $95 \pm 1$ & $96 \pm 2$ & $96 \pm 1$ & $94 \pm 3$ & $85 \pm 5$ \\
\hline \multirow{2}{*}{$\begin{array}{l}0.1 \mu \mathrm{M} \\
\mathrm{HOE} 140\end{array}$} & $\mathrm{pD}_{2}$ & $6.60 \pm 0.19$ & $6.38 \pm 0.14$ & $7.13 \pm 0.15$ & $6.32 \pm 0.19$ & $6.21 \pm 0.36$ & $6.24 \pm 0.17$ \\
\hline & $\mathbb{E}_{\mathrm{frax}}$ & $91 \pm 3$ & $84 \pm 5$ & $95 \pm 5$ & $82 \pm 4$ & $49 \pm 8+$ & $54 \pm \mathbb{1 1}$ \\
\hline \multirow{2}{*}{$30 \mu \mathrm{MNLA}$} & $\mathrm{PD}_{2}$ & $6.48 \pm 0.21$ & $6.20 \pm 0.16$ & $6.70 \pm 0.32$ & $6.70 \pm 0.32$ & $6.41 \pm 0.14$ & $5.91 \div 0.31$ \\
\hline & $E_{\text {mins }}$ & $77 \pm 7 \dagger$ & $72 \pm 10+$ & $81 \pm 10$ & $62 \pm 1.5 t$ & $74 \pm 84$ & $70 \pm 14 \hbar$ \\
\hline \multirow{2}{*}{$\begin{array}{l}\text { HOE } 140 \\
\text { WNLA }\end{array}$} & $\mathrm{pD}_{2}$ & $6.69 \pm 0.10$ & $6.11 \pm 0.10$ & ND & $6.21 \pm 0.10$ & $5.75 \pm 0.23$ & ND \\
\hline & $\mathbb{E}_{\text {ynas: }}$ & $69 \pm 10 t$ & $47 \pm 4+$ & ND & $69 \pm 11+$ & $32 \pm 10+$ & ND \\
\hline
\end{tabular}

Walues are shown as means $1518 M$ * P<0.05 versus $N P,+P<0.05$ versus control.

A

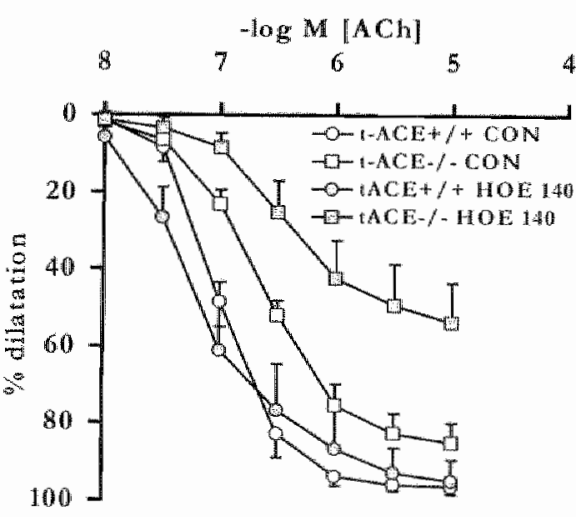

B

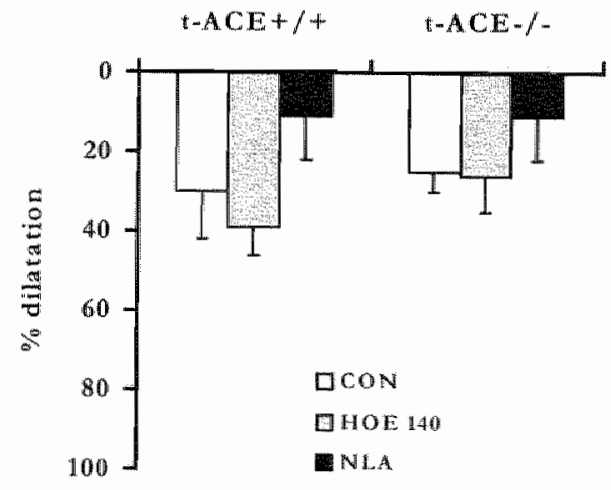

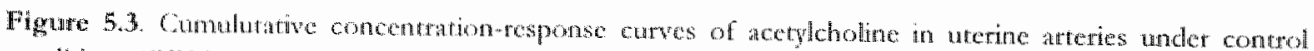
condition (GON: open symbols), and in the presence of 0 . 1 umol/L of the bradykinin 32 meceptor antagonist

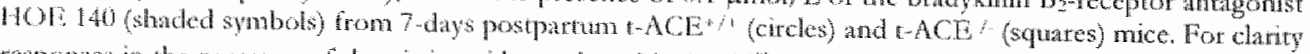

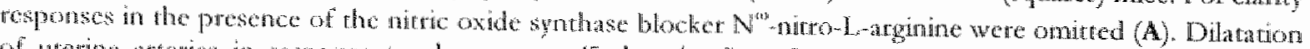

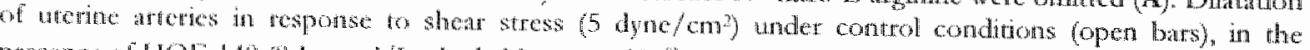

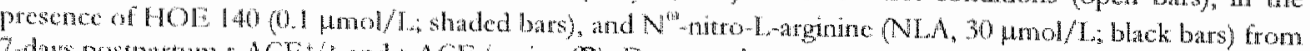

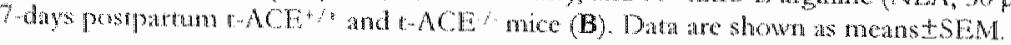




\section{Discussion}

This study demonstrates that bradykinin can be neleased by the arterial wall via chemical (acetylcholine-induced) and mechanical (shear stress-or flow-induced) stimulation, confrming previous observations of the important role of the kallikem-kinin systen in flow-dependent arterial diatation, thatough activation of bradykinin $B_{2}$-receptors coupled to endothelial NO release. ${ }^{37}, 23$ Moteover, tissue angiotensin-converting enzyme (tissue ACE) was found to mask a contribution of bradykinin to acetycholine-induced vasodlatation, and the role of bradykinin seemed to be modified during chronically altered local blood flow occurring during and after pregnancy.

We hypothesized that the contribution of bradykinin to flow-induced dilatation would be mote pronounced in uterine arteries from $\mathrm{t}-\mathrm{ACE} / \mathrm{mice}$, since angiotensinconverting enzyme is degrades bradykinin into smaller inactive peprides. The reason why we used late-pregnant and postpartum mice is twofold. Firstly, in parallel studies we studied the role of the kallikrein-kinin system and the renin-angiotensin system in uterine arterial structural responses to chronically increased blood thow during gestation and to a reduction in local blood flow after bith ( 7 days after pregnancy). ${ }^{223,224}$ Secondly, in settings of chronically elevated blood flow arterial flow-induced dilatation has been shown to be up-regulated, mainly by endothelium-deriwed vasoactive agents such as nitric oxide and prostacyclin. ${ }^{42}$

Responses to acetylcholine were simitar in uterine arteries of wild-type and $\mathrm{ACE}-\mathrm{A}$ mice, indicating that the release of endothelium-derived factors is preserved despite deficiency of tissue $\triangle \mathrm{CE}$. The presence of the bradykinin $\mathrm{B}_{2}$ receptor antagonist $\mathrm{HOE}$ 140 caused a significant reduction in the amplitude of vasodilatation only in uterine arteries of $t-A C E \%$ mice. This suggests that tissue ACE masks a contribution of btadykinin to acerylcholine-induced vasodilatation.

Uterine arteries responded to shear stress (5 dyne/ $\mathrm{cm}^{2}$ ) by vasodiatation. Flowinduced dilatation tended to be higher in uterine arteries of $t-A C F-$ mice compared to theit wild-rype counterparts and blockade of bradykinin B2-receptors reduced flowinduced dilatation to a latger extent in uterine arteries of $\mathrm{t}-\mathrm{ACE}$ mice. This indicutes that tissue ACE interferes with the function of shear stess-induced bradykinin release, probably by breakdown of the peptide. These obserntions futhemore confirm the important role of bradykinin as a shear stress-sensitive endothelial aurocrine factor. 37,22,

Pregnancy is known to increase uterine arterial nitric oxide production in tars ${ }^{\text {s. }}$ and humans. ${ }^{301}$ Expression of endothelial nitric oxide synthase (ONOS) MRNA and protein are increased in ovine pregnancy, 37 as a result of of estrogen-, vascular endothelial grow th

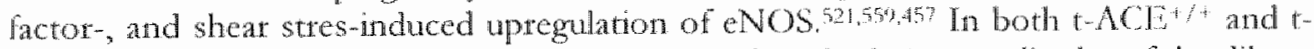
ACE mice, pregnancy was not accompanied by alrered relative amplitudes of the dilatot responses to acetylcholine. There are conflicting reports in the literature stating that acerylcholine-induced vasodilatation of pre-constricted uterine arteries can eithet be increased ${ }^{300,39381}$ or unchanged. 273,354 Methodological differences may account for the observed inconsistencies. For instance, the experimental ser-up of the pressure-myograph (arteriograph) differs from the wire-myograph. Diameter changes are recorded in the arteriograph, whereas contractile forces, dependent on the arterial wall mass, are measured in the wire-myograph. In addition, the level of pte-constriction prior to acorylcholine 
administration is not always similar in these studies. However, the nature of the endothclium-dependent dilator responses was cleatly altered in pregnant mice. In vessels of late pregiant $\mathrm{t}-\mathrm{ACE} \mathrm{E}^{+/}$mice, the inhibitory effect of $\mathrm{N}^{\mathrm{\omega}}$-nitro-L-arginine on both agonist-and shear stress-induced wasodilatation was larger when compated to responses in nonpregnant $\mathrm{A}-\mathrm{ACE} \mathrm{C}^{+}$- mice, suggesting that more nitric oxide is released in uterine arteries of late pregnant mice in response to acetylcholine, confirming previous observations by our group.23 In uterine arteries from late pregnant $t$ - ACE - mice, however, bradykinin seemed to xeplace the role of nitric oxide, since $\mathrm{HOE} 140$ caused a more than $50 \%$ inhibition of the acetylcholine-mediated vasodilatation, which was only matginally reduced by $\mathrm{N}^{(t)}$-nitro-I-arginine. The same trend was observed during shear stress-induced vasodilatation in uterine arteries from late pregnant $\mathrm{t}-\mathrm{ACE} / \mathrm{mice}$. These observations suggest that during late pregnancy tissue ACE is upregulated, reducing the contribution of endogenous bradykinin.

Flow-induced dilatation was markedly reduced in uterine arteties of 7 days postpartum mice, despite nomal acetylcholine-induced vasodilatation, suggesting a teduced shear stress sensing by the endothelium in these arteries. ${ }^{22}$ No clear role of bradykinin and nitric oxide were observed in these arteries.

Taken together, we confirm the impottant tole of bradykinin as a vasoactive agent that can be released by the endothelial cells as an autocine factor via agonist and shear stress. This is more pronounced during deficiency of tissue ACE. During physiologically increased blood flow (at tem pregnancy) the role of bradykinin seems to be masked by an upregulation of nitric oxide and $A C E$ in wild-rype uterine arteries. This in contrast to tACE / mice, in which the enhanced bradykinin levels appear to be sufficient for the flowinduced vasodilatation. 
Chapter 6

\section{Changes in Vascular Distensibility During Angiotensin-Converting Enzyme Inhibition Involve Bradykinin Type 2 Receptors}

Wendy M. Aartsen1, Rob H.P. Hilgers", Paul M.H. Schiffers', Mat J.A.P. Daemen², Jo G.R. De Mey', Jos F.M. Smits'.

Department of Phamacology \& Toxicology, and 2parinology, Catdiovascular Reseatch Institute Mastricht (CARM), Universiteit Maastricht, the Netherlands.

Based on: J Vasc Rer 2004;41:18-27. 


\section{Abstract}

Objective. Changes in arterial elasticity and structure occur during cardiovascular diseases and can be modified by angiotensin-converting enzyme (ACE) inhibitors. In the present study we investigated the role of tissue-bound $A C E(t-A C E)$ in the regulation of arterial morpho-mechanical properties.

Methods and Results. Large and small arteries of $\mathrm{t}-\mathrm{ACE} /$ mice were isolated to determine the passive pressure-diameter relationship. We observed that arteries of $t-A C E$ 1. mice exhibit a reduced arterial distensibility compared to $\mathrm{t}-\mathrm{ACE} \mathrm{E}^{t /+}$. This reduced arrerial distensibility was also observed after 9 weeks of captopril treatment $(80$ $\mathrm{mg} / \mathrm{kg} /$ day). We hypothesized that bradykinin type 2 receptor $\left(\mathrm{B}_{2}\right)$ stimulation might be involved in the regulation of arterial elasticity. $\mathrm{t}-\mathrm{ACE} / \mathrm{-and} \mathrm{t}-\mathrm{ACE}+/+$ mice were treated with the $B_{2}$ antagonist HOE $140(1 \mathrm{mg} / \mathrm{kg} /$ day) for 14 days. After HOE 140 treatment, botll the structural and mechanical changes observed in the $\mathrm{t}-\mathrm{ACE} \%$ carotid attery were abolished. Although HOE 140 administration increased blood pressure in both groups by approximately $10 \mathrm{mmHg}$, the pressure difference between the two groups was similar.

Conclusion. Tissue-ACE is involved in the regulation of arterial distensibility. The changes observed in $\mathrm{t}-\mathrm{ACE} \%$ mice ate not caused by an altered fetal development. Moreover, it is likely that the regulation of arterial distensibility by ACE involves stimulation of the $\mathrm{B}_{2}$ receptor. 


\section{Introduction}

In cardiovascular diseases like hypertension, ${ }^{36,453,467}$ myocardial infarction, ${ }^{3+4}$ heart failure, ${ }^{193,374}$ and atherosclerosis $83,3,3,570$ the arterial structure and arterial mechanics are altered. Since several of these alterations are reduced or prevented by angiotensinconverting enzyme (ACE) inhibition ${ }^{237371.36,453}$ or angiotensin type 1 receptor antagonism, ${ }^{415}$ the involvement of the renin angiotensin system (RAS) in the regulation of arterial structure and mechanics has been suggested.

$A C E$ is one of the central enzymes of the RAS and is responsible for the production of angiotensin II. In the cardiovascular system, ACE appears as a circulating enzyme in the blood and as an ecto-enzyme attached to the cell membranc. The importance of its position on the ceil membrane for the regulation of blood pressure and renal function has been demonstrated in mice lacking the membrane- or tissuc-bound ACE (t-ACE), ${ }^{141}$ Moreover, lack of $\mathrm{t}-\mathrm{ACE}$ changed the remodeling of pulmonary arteries during chronic hypoxia. ${ }^{568}$ We hopothesized that ACE and especially $t-A C E$ is involved in the regulation of vascular characteristics. To test this hypothesis we investigated the structure and mechanics of the thoracic aotta, common carotid artery and first-order mesenteric resistance artery of mice exhibiting only the $\mathrm{N}$-terminal part of ACE, which is capable of proteolytic cleavage but incapable of binding to the cell-membrane $(\mathrm{t}-\mathrm{ACE} / \mathrm{)}$. Arterial characteristics determined in witro wete compared to those of wild-type $(\mathrm{t}-\mathrm{ACE}+1 /)$ mice. To evaluate whether the lack of $t$-ACE during pre- and postnatal development results in altered adult arterial characteristics, we included a second experimental group. $t-A C E E^{1 /+}$ mice were treated with the ACE inhibitor captopril from 3 to 12 weeks after birth and their arterial chatacteristics were compared to arteries from untreated $\mathrm{r}-\mathrm{ACE}+/+$ mice. ACE is, apart from a prominent component of the RAS, also a component of the kallikrein-kinin system and responsible for the degradation of bradykinin. During ACE inhibition both the production of angiotensin $I I$ and the degradation of bradykinin are reduced.

We hyporhesized that stimulation of the bradykinin type $2\left(\mathrm{~B}_{2}\right)$ receptor might play an important role in the arterial alterations that occur during $A C E$ inhibition. The involvement of the $B_{2}$ receptor in the artetial morpho-mechanical alterations during $\mathrm{ACE}$ inhibition was investigated by comparing the arterial structure and elasticity of $\mathrm{t}-\mathrm{ACE} \mathrm{C}^{\mathrm{t}}$ and $\mathrm{t}-\mathrm{ACE} /$ mice with or without chronic (14 days) treatment with the $\mathrm{B}_{2}$ receptor antagonist HOE 140.

\section{Materials and Methods}

\section{Anthals}

Mice Lacking $1 A C E$. The generation of mice with a C57Bl6/129/SV generic background and lacking $\mathrm{t}-\mathrm{ACE}$ has been described by Esther and colleagues. ${ }^{141}$ Mice heterozygous for the mutated allele ( $\mathrm{t}-\mathrm{ACE}^{+/}$) were bred to obtain mice homozygous for the mutated allele $(\mathrm{t}-\mathrm{ACE} / \mathrm{t})$ and their wild-type littermates $\left(\mathrm{t}-\mathrm{ACE} \mathrm{A}^{+/+}\right)$. To genotype pups, genomic DNA was isolated from the tail using the Qiagen DNA isolation kit (Qiagen, Hilden Germany), Ready-to-go PCR beads (Amersham Pharmacia Biotech) were used for PCR analyses on 
genomic DivA. The three primers used are described by Esther and colleagues. la $^{\text {Age- }}$ matched adult (6-10 12 -month old) female mice ( $\mathrm{t}-\mathrm{ACE}+*$ and $\mathrm{t}-\mathrm{ACE} / \mathrm{C}$ ) were used to investigate the effects of $\mathrm{t}-\mathrm{ACE}$ deficiency on the peripheral arterial structure and mochanics.

Captoprithreatod Mice. Male and female wild-type mice at the age of 3 weeks had access to cither tap water or captopril dissolved in tap water $(80 \mathrm{mg} / \mathrm{kg} / \mathrm{day}$, Sigma-Aldrich, Stemhem, Germany) until they reached the age of 12 weeks. Female mice were used to investigate the effects of postnatal ACE unhibition. Male mice were used to detemine conscious blood pressure.

HOE 140.Treated Mice. At the age of 12 weeks mice were anesthetized with ketamine $(50$ $\mathrm{mg} / \mathrm{kg}$ i.m.) and xylazince (5 mg/ $\mathrm{kg}$ s.c.) and osmotic minipumps (Alzet 2002 ; Alza, Palo Alo, CA, USA) filled with $\mathrm{HOE} 140(1 \mathrm{mg} / \mathrm{kg} / \mathrm{day}$, Sigma-Aldrich, Steinheim, Gemany) dissolved in saline were subcutaneously implanted at the back of the neck. Mice were eicher undreated or treated with $\mathrm{HOE} 140$ for 14 days.

\section{Bhoud Pressur Measurament}

Conscious blood pressure was determined in untreated, captopril-treated or HOE 140 treated male $-A C E+/$ and $-A C F /$ mice. At 4 days before the end of treatment, mice were anaesthetized with pentobarbital $(110 \mathrm{mg} / \mathrm{kg})$ and placed on a heating pad to maintain body tempenature at $37^{\circ} \mathrm{C}$ to implant the catheters. One saline-filled catheter $(P E-25)$ was placed in the abdominal aotta via the fomoral artery to record systemic arterial pressure; a second saline-filled catheter ( $\mathrm{PE}-10)$ was placed in the jugular vern for infusions. Both catheters were subcutaneously tunneled to the neck and fixed.

Four days after surgety, the arterial catheter was connected to a pressure transducer (Micromitch, model 156PC 156 WI. Honeywell, Amsterdam, the Netherlands). Blood pressure signals were recorded and 10 min of stable recording were used to determine mean arterial pressure (MAP) at test. To determine ACE inhibition in captopril-treated mice, angiotensin I and II (0-35 ng, dissolved in saline) were cumulatively infused via the wenous carheter while measuring the MAP. Each dose was injected at the time of maximal MAP to avoid tachyphylaxis. Dose-response curves for angiotensin I and II were compared to untreated mice. In HOB 140 -reated mice, blockade of the $B_{2}$ receptor was established via a bolus injection (i.v.) of bradykinin $(25 \mathrm{ng})^{\circ}$ dissolved in $10 \mu$ of sterile water whilc recording $\mathrm{MNP}$.

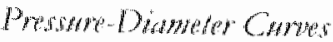

3- 10 -mm segments of first-otder mesenteric resistance arteries wete isolated. Arteries were ransferted to an organ chamber (arteriograph system, Living Systems Tustrumentation, Burlington, VT, USA). One end of the segment was mounted onto the proximal glass micropiperte. After fushing the artery with calcium-tree modified Krebs Ringer buffer (composition in mmol/L: $144 \mathrm{NaCl}, 4.7 \mathrm{KCl}, 1.2 \mathrm{MgSO}+1.2 \mathrm{KH} \mathrm{PO}_{4}, 14.9$ HEPES and 5.5 glucose, pH 7.4) the distal part of the segment was closed with a nylon knot $10 \mu m o l / 1$ Na-nitroptusside (SNP) was added to the organ chamber to assure maximal vasodilatation. The cantulated arterial segment was pressurized using foedbackcontrolled pressuro source (I.iving Systems Instrumentation) and placed under a binoculat lens connected to a video camera for recording of arterial diameter. A pressure-diameter 
(P-D) relationship was established by recording the diameter during stepwise $(10 \mathrm{mmHg})$ increases in intraluminal pressures from 20 to $140 \mathrm{mmHg}$. After each step the vessel was allowed to equilibrate until a stable diameter was reached.

A similar procedure was followed for the thotacic aorta and common carotid artery. However, these arteries were only cannulated at one end while the other end was closed (blind sac). Due to the intransparancy of the thoracic aorta and carotid artery, outer diameters were recorded. For $\mathrm{t}-\mathrm{ACE}-/$ and $\mathrm{t}-\mathrm{ACE}^{+/}$mice, the thoucic aorta, carotid and mesenteric resistance artery were used, while the carotid and mesenteric resistance artery were used from untreated $t-\mathrm{ACE}^{+/+}$and $t-A C E^{+/}$mice treated whth captopril. In untreated $\mathrm{t}-\mathrm{ACE}+/ 4$ and $\mathrm{HOE} 140$ meated $\mathrm{t}-\mathrm{ACE}$ * mice, the $\mathrm{P}-\mathrm{D}$ relationship of the carotid artery was determined.

\section{Morphoryetry}

Vessels were formalin-fixed at $80 \mathrm{mmHg}$ in the arteriograph. Fixed vessels were embedded in paraffin and cross-sections (4 $4 \mathrm{~m})$ were stained with Lawson's solution (Boom, Meppel, the Netherlands) to visualize the internal and extemal elastic laminac. Video images were made from cross-sections using a Zeiss axioscope and a standard charge-coupled device camera (Sony). Using I AVA sofwate OAVE 1.21, Jandel Scientific, Corte Madera, CA, USA) the circumference of the internal and external elastic laminae were determined. From these values the media cross-sectional area (mCSA) was calculated. To obtain the elastin/collagen ratio, cross sections were deparaffinised and ncubated in phosphomolybdenic acid $(0.2 \%)$ for 5 minutes, followed by incubation with Sirtus Red (0.1\%) in saturated picric acid solution for 90 minutes. After washing with 0.1 $\mathrm{MHCl}$ for 2 minutes, sections were dehydrated and protected with coverslips. 28 Collagen content was measured at 400X magnificarion in the aortic media OAVA 1.21, Jandel Scientific).

\section{Calcriation of Paraneters}

Arterial distensibility or the relative change in arterial lumen volume induced by a given increase in intraluminal pressure was estimated by: $\mathrm{DC}=\Delta \mathrm{D}_{\mathrm{n}} /\left(\left(\mathrm{D}_{\mathrm{n}} \cdot 1\right) / 2\right) \cdot \Delta \mathrm{P}(\mathrm{DC}=$ distensibility; $\mathrm{D}_{\mathrm{n}}=$ outer diameter at a given intraluminal pressure; $\mathrm{P}=$ intraluminal pressure) ${ }^{85}$ For the mesenteric resistance artery citcumferential wall stress and incremental elastic modulus were also calculated. ${ }^{36}$ Circumferential wall stress ( $\sigma$ ) is related to wall thickness (b) and wall tension ( $T$ ): $\sigma=\mathrm{T} / \mathrm{h}$. According to Laplace's llaw, $T$ depends upon transmural pressure $(P$,$) and radius (r): T=P_{i} r$. Incremental dasuc

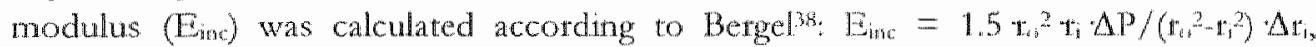
which derives from $0.75 \mathrm{D} / \mathrm{h} \cdot \mathrm{DC}$, where $\mathrm{r}_{\mathrm{s}}=$ outer radius; $\mathrm{r}_{\mathrm{i}}=$ inner radius, and $\mathrm{D}=$ outer diameter.

\section{Statistics}

Data are expressed as meantSEM. A Student t-rest was used to test the impact of the generic modification, captopril or HOE 140 treatment on blood pressure. The impact of the generic modification, captopril or HOE 140 treatment was tested with a twomway 
ANOVA for repeated measurements in case of the P-D curves. $P<0.05$ was considered statistically significant.

\section{Results}

\section{Gemeral Obientahons}

Age-matched adult $t$-ACE/ mice (male and female) were significantly lower in body weight compared to t-ACE $+1+$ mice (Table 6.1 and 6.2 ). Also body weight of young ${ }^{+}$ $A C E * / 4$ (both sexes) mice treated with captopril for 9 weeks was significantly lower than

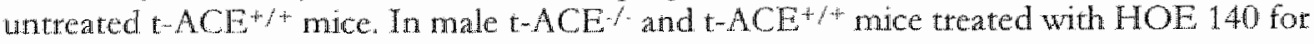
14 days abolished the difference in body weight.

Hypotension ( $80 \pm 3$ versus $110 \pm 4 \mathrm{mmHg}$, Table 6.1) was observed in $\mathrm{ACE}-\mathrm{A}$ mice. HOE 140 treatment increased MAP with $10 \mathrm{mmHg}$ in both $\mathrm{m}-\mathrm{ACE} / \mathrm{and} \mathrm{t}-\mathrm{ACE}+\mathrm{m}$ mice without changing the blood pressure difference of approximately 30 mm Hg between the two groups. Heart tate was unaffected by $\mathrm{A}-\mathrm{CE}$ deficicncy or antagonist treatment.

Table 6.1. General pameters in male $1-A C E+/+$ and $t-A C F-$ - mice, wnteated or treat with waptopil ot lom 140

\begin{tabular}{|c|c|c|c|c|c|c|}
\hline & $-\mathrm{ACE}+/+$ & $A C B^{-}$ & $\mathrm{ACE}$ & $\begin{array}{l}\text { T-ACEN+ } \\
\text { Captogril }\end{array}$ & $\begin{array}{l}\mathrm{ACE} \\
\mathrm{HOE} / 40\end{array}$ & $\begin{array}{l}\text { TACE } \\
\text { MOE } 140\end{array}$ \\
\hline Gender & male & male: & matc & malc & male & male \\
\hline Age & 6-12 mo & $6-12$ mo. & $12-14 \mathrm{wks}$ & 12-14 whs & $12-14 \mathrm{wks}$ & $12-14$ wks \\
\hline Number & 7 & 7 & 7 & 5 & 6 & 5 \\
\hline Body wetght (g) & $30+1$ & $26+2$ & $28+1$ & $33 \pm 1+$ & $26 \pm 1$ & $26 \pm 1$ \\
\hline$M A P(m m H g)$ & $110 \pm 4$ & $80 \pm 3$ & $111+2$ & $80=7$ & $120+5$ & $87 \pm 8 \neq$ \\
\hline Heatst tale (bpm) & $662+14$ & $657 \pm 19$ & $550+30$ & $600+48$ & $673 \pm 32$ & $612+50$ \\
\hline
\end{tabular}

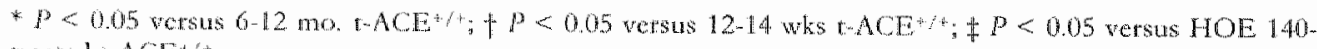
troted $\mathrm{ACH}+\mathrm{CH}$.

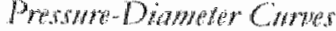

Picessurc-Dameter ( $\mathrm{D}-\mathrm{D})$ curves and calculated arterial distensibility of the thotacic aotta (TA), common carotid artery (CA), and firstorder mesenteric resistance artery (MrA) of adult age-mathed female $\mathrm{t}-\mathrm{ACE} /$ and $\mathrm{t}-\mathrm{ACE} / \mathrm{-mice}$ are shown in Figure 6.1 . CA and MrA of youmg uncreated and capropril-treated $\mathrm{t}-\mathrm{ACE}+\mathrm{t}$ female mice were used to construct $P-D$ curves and to (-igure 6.2). The P-D curves of the "TA and the $C A$ show a rypical S-shape comparable to the curves described by Bergel obtained in the TA (blind sac). ${ }^{3 *}$ The P-D curves of the MrA were constructed in a situation of fixed length and show a typical progressive decrease of distensibiliry is In $t$-ACE. vessels the maximal diameter was reached at a lower pressure compared to $t-A C E^{-4}$ ressels. This is reflected in the distensibility, which is shifted to the left in the case of the TA and CA, resulting in a significanty lower distensibility between 80 and $120 \mathrm{~mm} / \mathrm{g}$. The distensibility of the $\mathrm{Mr} A$ was consistently lower in $\mathrm{A}-\mathrm{ACE} / \mathrm{mice}$.

After prolonged captopril treament ( $80 \mathrm{mg} / \mathrm{kg} / \mathrm{day}$ ) a comparable effect on the MrA distensibility was observed in t-ACE arteries. Figure 6.3 shows the incremental 
elastic modulus ( $\mathrm{E}_{\text {inc }}$ ) as a function of the circumferential wall stress for both the $\mathrm{C}-\mathrm{ACE}$ -

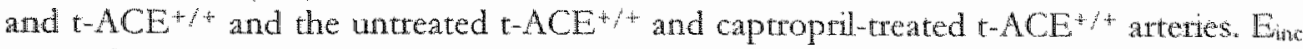
was only calculated for the MrA because wall thickness of the TA and CA could not be wisualized. In both $\mathrm{MrA}$ of $\mathrm{t}-\mathrm{ACE} /$ and captopril-treated mice, Em was elevated compared to the $\mathrm{MrA}$ of $\mathrm{t}-\mathrm{ACE}+/$ mice.

A reduced distensibility was not only seen in CA from female mice, but also CA of male $t-A C E /$ nice showed a reduced distensibility compared to $C A$ of $+A C E$ male mice (Figure 6.4). After treating $\mathrm{t}-\mathrm{ACE}^{+/ t}$ mice with $\mathrm{HOE} 140(1 \mathrm{mg} / \mathrm{kg} / \mathrm{day}$ ) for 14 days, no difference in the $\mathrm{P}$-D curves could be observed (Figute $6.4 \mathrm{~A} / \mathrm{B})$. In $\mathrm{T}-\mathrm{ACE}$ mice, HOE 140 treatment changed the $\mathrm{P}$-D curve of the $\mathrm{CA}(\mathrm{Figure} 6.4 \mathrm{~A} / \mathrm{B}$ ) and distensibility curves (Figure $6.4 \mathrm{C} / \mathrm{D}$ ); both curves were shifted towards the $\mathrm{A}-\mathrm{ACE}^{+/ t}$ curves. HOE 140 treatment had no effect on the distensibility curves of the CA of ACE + mice (Figure $6.4 C / D)$.

Table 6.2. Morphometry of wessels fixed at $80 \mathrm{mmHz}$

\begin{tabular}{|c|c|c|c|c|c|c|c|c|}
\hline Gonorype & Gender & Age & $B W(g)$ & $\mathrm{n}$ & $\begin{array}{l}\text { medis CSA } \\
\left(\mathrm{x} 10^{\mathrm{9}} \mathrm{km}^{2}\right)\end{array}$ & $\begin{array}{l}\text { Radius } \\
\text { (um) }\end{array}$ & $\begin{array}{l}\mathrm{Mt} \\
(\mu \mathrm{rat})\end{array}$ & $\begin{array}{l}\text { WL } \\
\times 100 \\
\end{array}$ \\
\hline \multicolumn{9}{|l|}{ Thomal Monta } \\
\hline $\mathrm{LACE}+\%$ & Female & 6-12 mo. & $28+1$ & 6 & $123+8$ & $454 \pm 6$ & $41+2$ & 0.010 .5 \\
\hline $\mathrm{T}-\mathrm{ACE}$ & female & $6-12 \mathrm{mo}$ & $23 \pm 1 *$ & 5 & $91 \pm 1 *$ & $437 \pm 17$ & $32 \pm 1 *$ & $7.4+0.6$ \\
\hline \multicolumn{9}{|l|}{ Conotianthes } \\
\hline $\mathrm{T}-\mathrm{ACE}$ & temale & $6-12 \mathrm{no}$ & $28+1$ & 5 & $20 \div 2$ & $212 \pm 6$ & $14 \pm 1$ & $6.6 \pm 0.3$ \\
\hline $\mathrm{CACE}$ & Female & $6-12 \mathrm{mo}$ & $23 \pm 1 *$ & 5 & $19+2$ & $209+5$ & $14+1$ & $6.6 \pm 0.7$ \\
\hline $\mathrm{ACE}$ t untreated & female & $12-14 w k s$ & $22+1$ & 7 & $17 \pm 1$ & $192+3$ & $14 \pm 1$ & $7.1 \pm 0.6$ \\
\hline$-A C E++$ captopril & female & $12-14 w k s$ & $19 \pm 1$ * & 8 & $12+1$ 皮 & $199+5$ & $9+1 *$ & $4.8 \pm 0.6$ 的米 \\
\hline$\left(-z^{4} \mathrm{CF}+\%\right.$ & male & $12-14$ wks & $28 \pm 1$ & 6 & $20 \pm 1$ & $202+5$ & $15 \pm 1$ & $7.6 \pm 0.1$ \\
\hline$C-A C D F$ & male & $1214 w k s$ & $23 \pm 1 *$ & 6 & $14 \pm 1 *$ & $196 \pm 1$ & $11 \pm 1:$ & $5.6 \pm 0.3 \%$ \\
\hline $\mathrm{ACE}+\mathrm{HOD} 140$ & maks & $12-14 x+28$ & $26 \pm 1$ & 7 & $20 \pm 3$ & $209+15$ & $15 \pm 1$ & $7.6 \pm 0.1$ \\
\hline$M C E \%$ HOE 140 & male & 12-14 wks & $26 \pm 1$ & 5 & $17 \pm 1$ & $191+4$ & $14 \pm 1$ & $7.2 \pm 0.7$ \\
\hline \multicolumn{9}{|l|}{ Aferenatoric Arrery } \\
\hline$M A C F$ & Conmale & $6.12 \mathrm{mo}$ & $28 \pm 1$ & 7 & $32+0.3$ & $108 \pm 0$ & $4.6 \pm 10.3$ & 4.310 .2 \\
\hline $\mathrm{ACE}$ & temate & $6-12 \mathrm{mo}$ & $23 \pm 1$ & 7 & $4.0+0.5$ & $129+8$ & $4.7 \pm 04$ & 3. $1+0.5$ \\
\hline 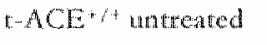 & female & $12-14 \mathrm{wks}$ & $22 \pm 1$ & 6 & 2.450 .4 & $30+14$ & $46 \pm 06$ & $5,8 \pm 0.6$ \\
\hline t-ACE + captopril & fenrale: & $1214 \mathrm{wks}$ & $19 \pm 1$ k* & 9 & 2.390 .1 & $93+3 *$ & $39 \pm 0.1$ & $4.3 \pm 0.2$ \\
\hline
\end{tabular}

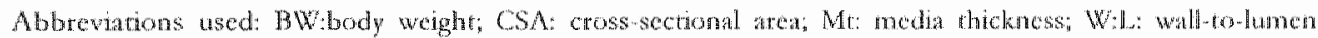

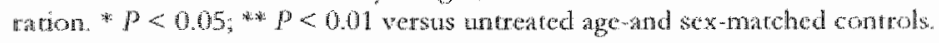

\section{Monphomeny}

Four parameters of arterial structure were detemined and are presented in Table 6.2. In adult female $t-A C E$ \% mice no significant differences in $C A$ and $M A-A$ structure were found compared to $t-A C E / 4$. In the TA of $t-A C E$ mice, media cross-sectional area (CSA) and medial thickness (Mt) were significantly reduced but did not result in a significantly lower wall-tommen ratio (W:L). 
A

Thoracic Aorta

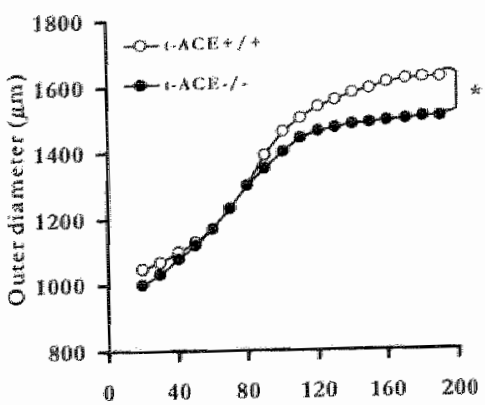

C

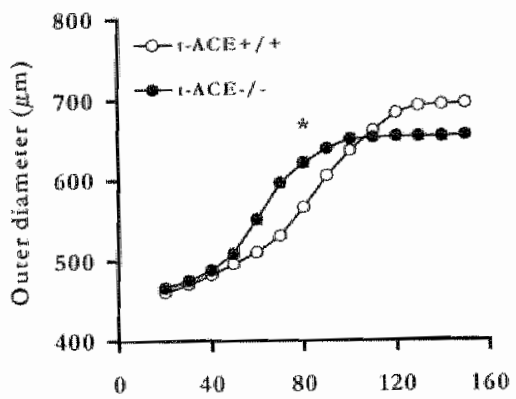

E

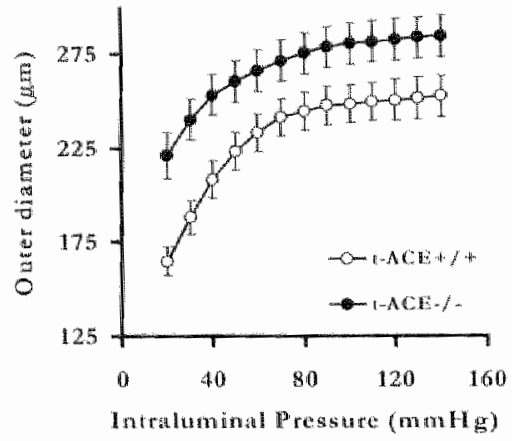

B

Thoracic Aorta

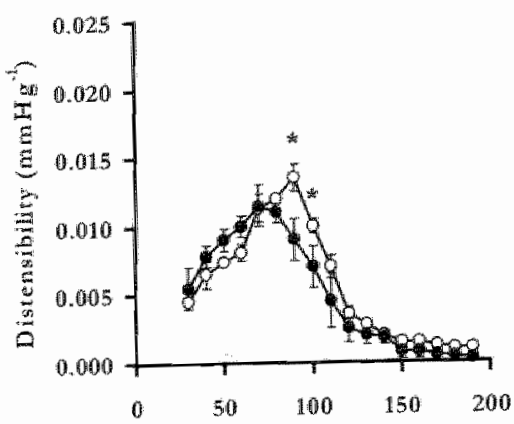

D

\section{Carotid Artery}

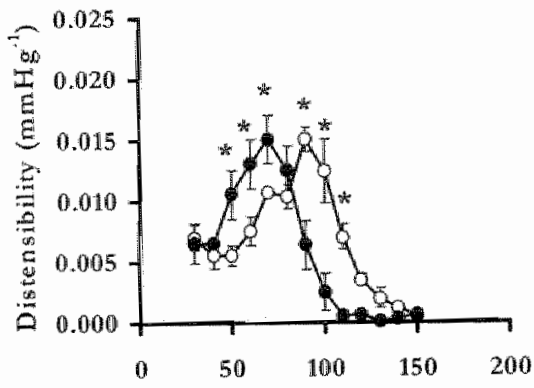

$\mathbf{F}$

Mesenteric Resistance Artery

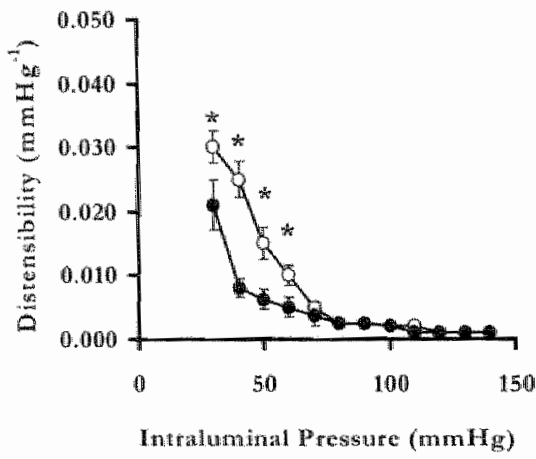

Figure 6.1. Relationship between imposed intrahumal pressure and dimeter in isolated thomeic aora (A),

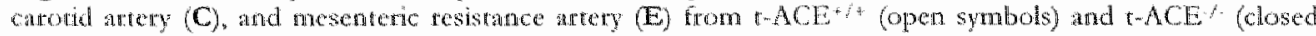
symbols) mice. Calculated distensibiliny for the thoracic arta $(\mathbb{B})$, carotid artery $(D)$, and nesenteric resistance

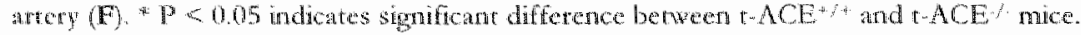


Prolonged reatment with captopril resulted in s significant reduction in CSA and Mt in the $C A$, while the radius was unchanged. The opposite effect was observed in the MrA: the radius was enlarged without changing CSA and Mr, resulting in a reduced W:L in both the CA and MrA. A reduced W:L caused by a snaller CSA and MT withour an altered radius was also observed in the $C A$ of young male $A C E$ mice. After 14 days of HOE 140 treatment the W:L of the $\mathrm{CA}$ of male $-\mathrm{ACE} /$ mice nomalised.

Composition of the arterial wall was determined by measuting the collagen and elastin content. Because of the small vessel size relable values for collagen and chastin percentages could only be obtained from aortic sections. The percentages of collagen $(12 \pm 1$ versus $12 \pm 1 \%)$ and elastin $(49 \pm 2$ versus $49 \pm 2 \%$ were sinilat in $T A$ of $t-A C E \%$ and $\mathrm{ACCE}+\mathrm{mice}$.

A

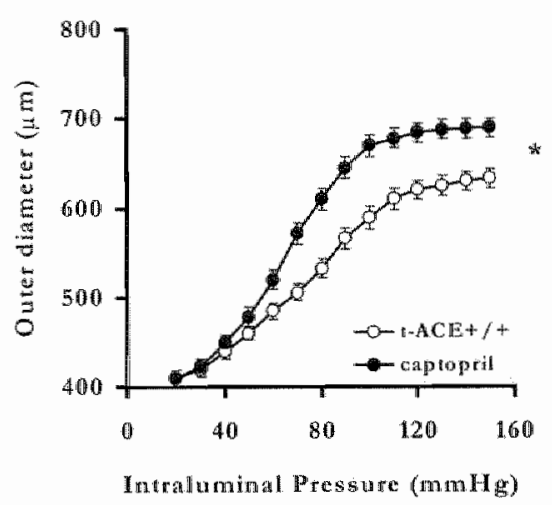

Carotid Artery

C

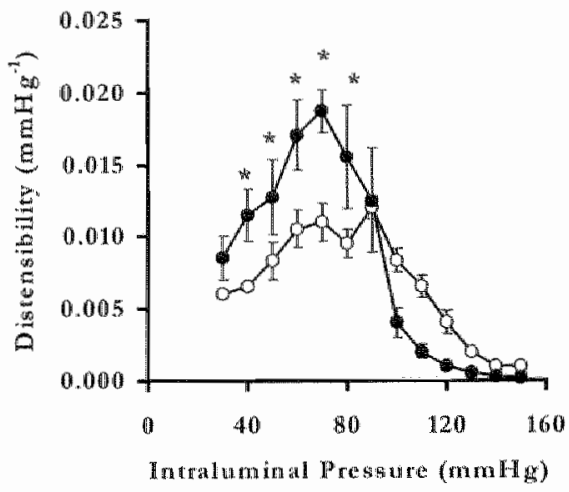

B

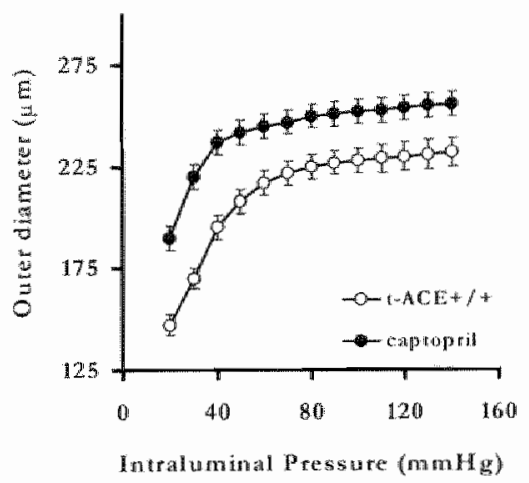

Mesenteric Resistance Artery

$\mathrm{D}$

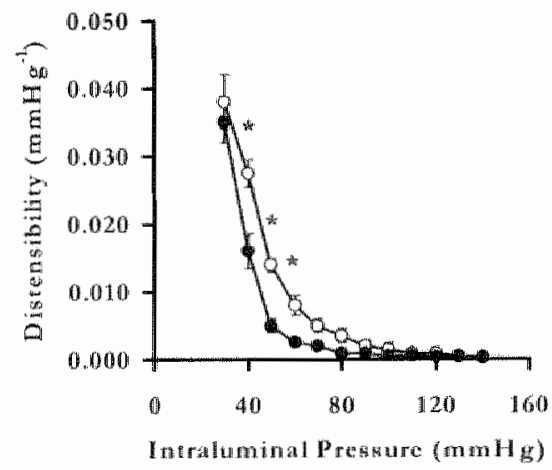

Figure 6.2. Relationship between imposed intratuminal pressure and diameter in isolated carotid anteries (A)

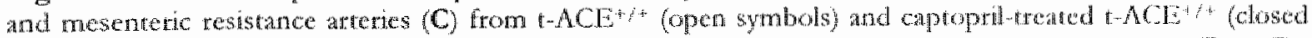
symbols) mice. Calculated distensibility for the carodd artery (B) and mesenteric resistance artery (D), * $P<$ 0.05 indicates significant difference between captopril-treated and untrated $1-A C F$, mice. 


\section{Discussion}

In the present study the effecs of $\mathrm{t}-\mathrm{ACE}$ deficiency, ${ }^{\text {in }}$ phamacological ACE inhibition and antagonism of $B_{2}$ receptors on the structure and mechanics of systemic arteries were investigated. $\mathrm{ACE}$ deficiency reduced distensibility of large and small vessels in male and female mice. Similar effects were obtained in large and small vessels isolated from t$\mathrm{ACE}^{+/+}$mice treated with captopril for 9 weeks. Since in these animals treatment was started after weaning, these data suggest that the vascular alterations found in $\mathrm{C}-\mathrm{ACE}$ \% mice are not due to $\mathrm{t}-\mathrm{ACE}$ deficiency during fetal development. Whether stimulation of $B_{2}$ receptors plays a significant role in the regulation of vascular distensibility was investigated by treating both $t-A C F$ and $t-A C E *$ mice with the $B_{2}$ teceptor antagonist $\mathrm{HOE} 140$ for 14 days. The results show that the reduced distensibilicy observed in $-\mathrm{ACE}$ 1 mice could be abolished by blocking $\mathrm{B}_{2}$ receptors.

The pressure-diamerer curves of the thoracic aorta, carotid and mesenteric resistance artery of $1-A C E^{-}$were different from those of $\mathrm{ACE}+\mathrm{A}$ - mice "The maximal diameter in t-ACE/ atterics was reached at a lower pressure compared to $t-A C F^{+}$arteries. This indicates a reduced distensibility in all three artery types. The calculated distensibility curves of the large arteries showed a significant leftward shift leading to a reduction in distensibility between $80-120 \mathrm{mmHg}$. The distensibility of the mesenteric tesistance artery of $t-A C E \%$ mice was consistenty lower than the distensibility of the mesenteric resistance artery of $\mathrm{t}-\mathrm{ACE}+/+$ mice.

Since $t-A C E$ - mice are lacking $t-A C E$ during the critical phase of fetal development, in which many RAS components, including $\mathrm{ACE}$, are highly expressed, $33 \%, 486$ the reduced vascular distensibility might be the tesult of an alteted fetal development. To investigate this hypothesis, $T-A C E$, mice wete treated with captopril from 3 to 12 weeks of age and their vascular distensibility was compared to untreated $t-A C E+/+$ nice.
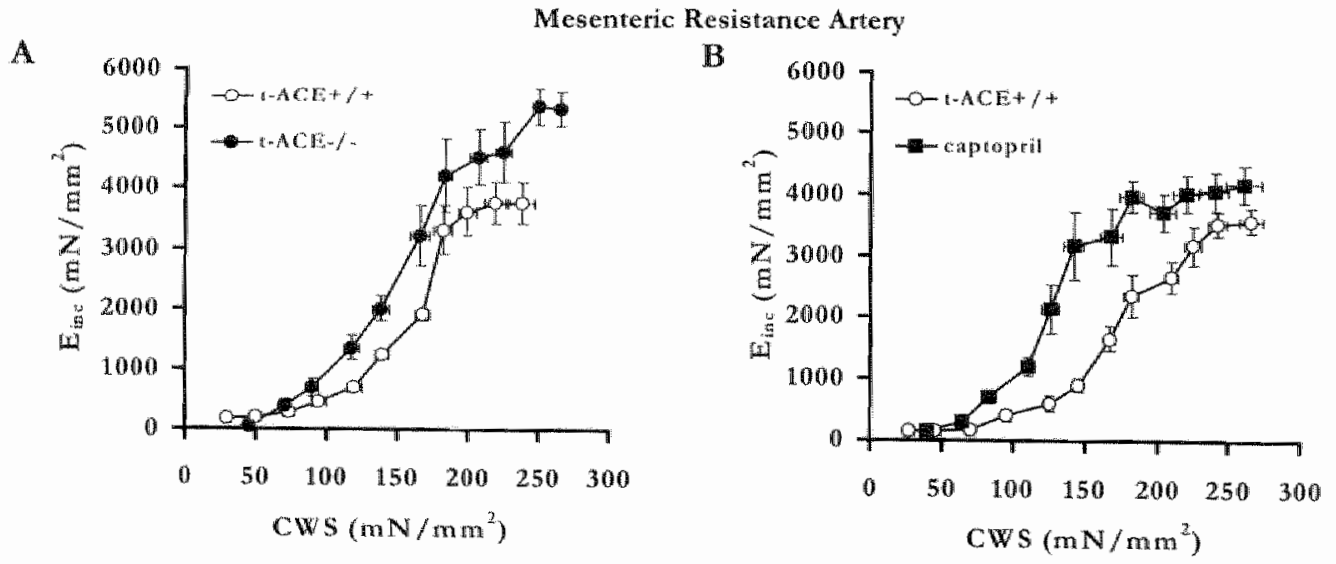

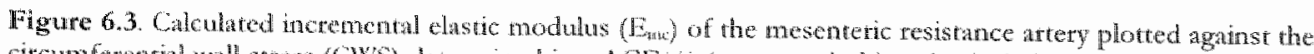

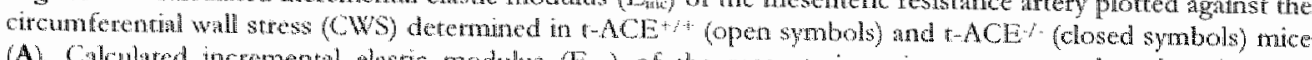

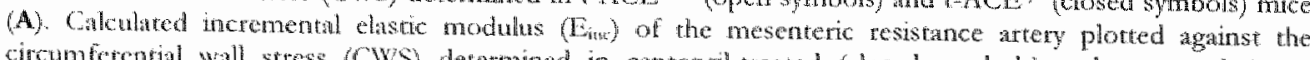

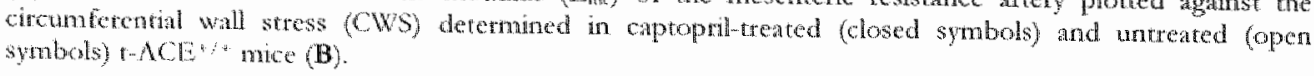


Effectiveness of the captopril treatment is demonstrated by blood pressure reduction. Moteover, an inhibited blood pressure increase after the infision of angiotensin I was observed in $\mathrm{t}-\mathrm{ACE}$ mice, while the pressot response to angiotensin II remained unchanged (data not shown). After prolonged captopnil treatment, a comparable reduction in distensibility was observed in the carotid and mesenteric resistance artery as observed in $\mathrm{t}-\mathrm{ACE} \%$ mice. These results suggest that the reduced vascular distensibility observed in $t$-ACE $\%$ mice is not due to an altered fetal development.

A

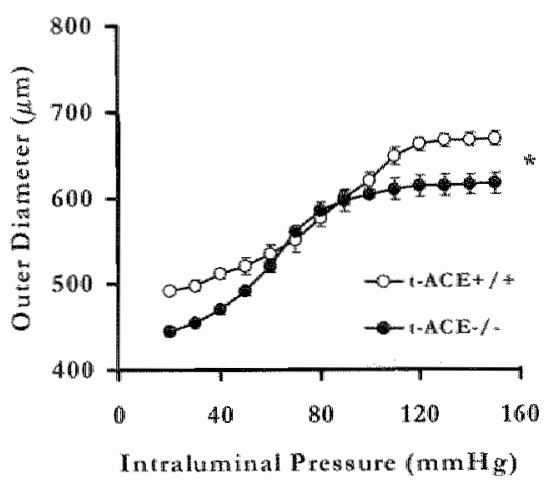

C

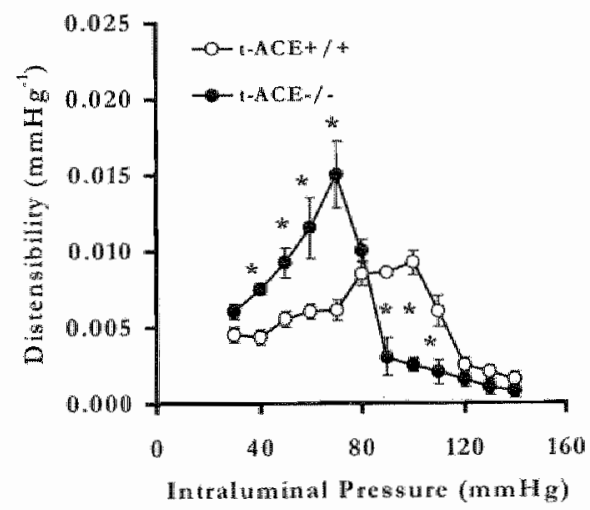

\section{Carotid Artery}

B

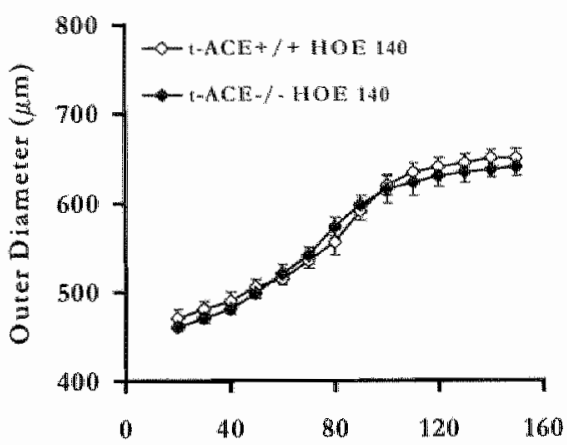

Intraluminal Pressure $(\mathrm{mmH})$

D

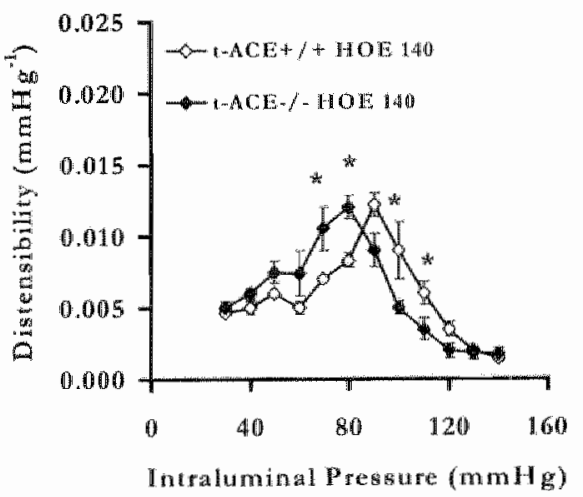

Figure 6.4. Relationship berween imposed intraluminal pressure and diameter in isolated carend arteries from H-ACE / (open symbols) and ACE (closed symbols) mice (A). Relatsonship berween imposed intraluminal pressure and diameter in isolated carotid arteries from $\mathrm{t}-\mathrm{ACE}, 4$ (open symbols) and $\mathrm{HCE}$ - (closed symbols) HOE 140 -reated mice (B). Calculated distensibility for carotid arterics from t-ACE $/ 1$ (open symbols) and $t$ ACE? (closed symbols) mice (C). Calculated distensibility for carotid arteries from t-ACE" " (open symbols) and $t-A C E$ (closed symbols) HOE 140 -treated mice (D). * $\mathrm{P}<0,05$ indicestes significant difference berwech $1-$ $\mathrm{ACE}+*$ and (untreated) $\mathrm{t}-\mathrm{ACE} /$ mice. 
Since the elastic properties of the arteries ate tested in who without fow or vasculat rone, the decreased distensiblity is directly related to the vessel wall composition. 38 , 467 . The elastic properties of maximally relaxed arteries depend on vessel wall components and wall geometry. Morphometric analysis of arteries from adult female $\mathrm{A}-\mathrm{ACE} / \mathrm{mice}$ showed. that only the medial CSA, Mt, and W.L of the thotacic aorta were decreased, while the motphomery of the carotid artery and mesenteric resistance artery was unaltered. In the captoptil-treated female mice, medial CSA, Mr, and W:L were reduced in the carotid artery, while the radius of the mesenteric resistance artery was significantly wider compared ro the untreated artery, resulting in a reduced W: $\mathrm{L}$.

By calculating the incremental elastic modulus (Enc), or the relationship between strain and stress, changes in the vessel wall motphometry, which affect vascular

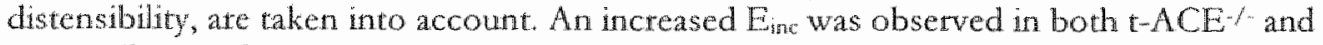
captopril-treated mesenteric resistance arteries. Although wall thickness of the carotid artery and thoracic aorta could not be visualized, it was determined afret fixation at 80 mmHg. Medial CSA and Mt of the thoracic aorta wete reduced in the absence of $t-A C E$. The morphomery of the carotid artery was similar in both $t-A C E-$ and $t-A C E+$ female mice. In captopril-treated mice and male $t-A C E-$ mice, the canotid artery medial CSA and Mr (and hence Wr) wete reduced without a change in the radius. In all artentes distensibility (DC) was reduced beween 80 and $120 \mathrm{mmHg}$. When DC is decreased and Wt is either equal ot reduced, $E_{\text {inc }}(\mathrm{D} / \mathrm{WT} \cdot \mathrm{DC})$ is increased. This implies that Einc is increased in all tested arteties of $\mathrm{ACE} / \mathrm{mice}$ compared to arteries of $\mathrm{t}-\mathrm{ACE} \mathrm{C}^{\mathrm{t}+\mathrm{micc}}$ and in the captopril-reated carotid and mesenteric resistance arteries compared to the untreated carotid and mesenteric resistance artery. This incteased Eme reflects an increase in arterial stiffress.

These results suggest that ACE inhibition induces arterial stiffness, which seems to be paradoxical when compared to previous studies. Under several pathological circumstances such as hypertension, 31,46 myocardial infatction, 34 and atherosclerosis, 43 $\mathrm{ACE}$ inhibition enhances vascular compliance. The frst remark that should be made by comparing the results of the present study with ptevious observations is that the majority of these studies were performed in a siruation of cardiowascular disease, in which elevated RAS activity is likely. In the present study, the effects of either $t-A C E$ deficiency ot prolonged $\mathrm{ACE}$ inhibition on the arrerial compliance were investigated without obvious signs of cardiowascular discase. Notable are the studies by Baumbach and colleagues on cerebral arterioles in which they demonstrate that overexpression of human renin and angiotensinogen induces hypertrophy and an increased distensibility of these vessels. ${ }^{29}$ The opposite was demonstrated in spontancously hypertensive tats: hypertension decreased atterial stiffness, which could be abolished by the administation of an $A C E$ inhibitor. ${ }^{20}$ These results ane comparable to the increased atterial stiffess observed in the present sndy. A second tremark concens the fact that who distensibility could not

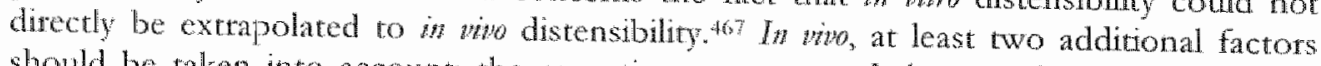
should be taken into accounc: the operaring pressure and the vasculat tone, 407 , 458 both influenced by angiotensin 11 and bradykinin.

To investigate the role of the $\mathrm{B}_{2}$ receptor in the vasculat distensibility changes observed during ACE inhibition, the pressure-diameter curves of the carotid artery of untreated and HOE 140 -treated male t-ACE/- and $-A C E$ - mice were compared. As in 
fernale twCE $\mathrm{ACH}$ - me pressure-dameter curve of the carotid artery of male $\mathrm{ACE}$. mice reached its maximal diameter at lower pressure than the catotid artery from ACE mice. Thus, in both male and female $t-A C E-$ mice, vasculat distensibility is reduced between 80 and $120 \mathrm{mmHg}$. A decreased distensibility accompanied by a reduced wall thickness while the diameter was unaltered will lead to an increased E Encin Reduced carotid artery wall thickness in $\mathrm{A}-\mathrm{ACE} / \mathrm{nice}$ was not observed in femate $\mathrm{ACE}$ - mice. This discrepancy might be due to the difference in age. Male $\mathrm{t}-\mathrm{ACE} /$ and $\mathrm{A}-\mathrm{CH}+\mathrm{H}^{+}$mice were treated with $\mathrm{HOE} 140$. A bohs injecrion of bradykinin $\left(25 \mathrm{ng}\right.$, ${ }^{10}$ which reduced blood pressure by approximately $30 \mathrm{mmHg}$ in untreated mice, did not affect blood pressure in HOE 140-treated $\mathrm{t}-\mathrm{ACE}$ / and $\mathrm{t}-\mathrm{ACE}+\mathrm{CH}^{+}$mice (data not shown), which indicates that the $\mathbb{B}_{2}$ receptors were effectively blocked. After 14 days of treatment with HOE 140 the difference in the pressure-diameter and distensibility curves of carotid arteries from $t-A C E /$ and $t-A C E+t+$ mice was no longer observed. "The distensibility curve of catotid anteries from $\mathrm{ACE}^{-}$mice was significantly shifted towats the distensibility curves of their wild-type connterparts. Morphometric analysis of catotid arteries from the treated $t-A C E /$ and $t-A C E \%$ mice revealed that the vascular geometry of the treated vessels was similar. Nomalization of the vascular geometry covild be related to the notmalization in body weight after HOE 140 treatment. Alterations in body weight and glucose metabolism during. ACE inhibirion have been described previously and the effects on insulin and glucose metabolism have been attributed to the elevated bradykinin lewels.216,217 After HOE 140 treatment, both the reduction in carotid artery distensibility and wall thickness were restored. Thus, the increased Eine observed in arteries of $\mathrm{C}-\mathrm{ACF}-\mathrm{H}$ mice can be abolished by 14 days treatment with HOF 140.

As in young rats, the blood pressure is affected by $\mathrm{HOF} 140$ treatment resulting in an increase of apptoximately $10 \mathrm{mmHg}$ compared to the blood pressure values for untreated $\mathrm{t}-\mathrm{ACE}^{-/}$and $\mathrm{t}-\mathrm{ACE} \mathrm{CE}^{+/ 2}$ mice ${ }^{334}$ The blood pressure difference of approximately $30 \mathrm{mmHg}$ between untreated $\mathrm{t}-\mathrm{ACE} /$ and $\mathrm{t}-\mathrm{ACE}+\mathrm{t}$ mice remained unaffected by 14 days of $\mathrm{HOE} 140$ treatment. Despite maintenance of this pressure diffefence arterial distensibilizy was restored by $\mathrm{HOE}$ 140, which suggests that the increased arterial stiffness observed in $-A C E /$ mice is not directy related to the blood pressure.

In the present study, the vascular distensibility was measured who whour fow or vascular tone. Therefore, alterations in distensibility are directly related to the vessel wall composition " ${ }^{\text {th }}$ Collagen and elastin densities were similar in aotas from $A C F /$ ard t$A C E^{+/ 2}$ mice, suggesting that the reduced distensibility found in $t-A C E$ mice is not due to a change in the elastin to collagen ratio. An altemative explanation for the reduced vascular distensibility might be found in the cell-matrix cross-bridges. For instance, cellmatrix cross-bridges between fibronection and the $\alpha \beta_{1}$ integrin receptor are tesponsible for the formation of a nework between all wall components. 45 Both angiotensin II and bradykinin are able to infuence the vascular fibrinolytic balance. $10,61,579$

In summary, ACE inhitition, either genetically- ot phamacologically-induced, results in atterial stiffness in mice. These arterial changes can be abolished by $B_{2}$ roceptor antagonism, which suggests that the $B_{2}$ receptor is involved in the increased arterial stiffness obserwed during $\mathrm{ACE}$ inhibition. However, the exact mechanism remains still unclear. Remarkable is that HOE 140 administation only affected carotid artery 
Chapter 609

distensibility of $t-A C E$ - and not $t-A C E$ - mice, suggesting that $B_{2}$ receptor antagonism does not affect arterial distensibility in general but only during teduced ACE activity. 
Chapter 7

\section{Tissue Angiotensin-Converting Enzyme in Imposed and Physiological Flow-Related Arterial Remodeling in Mice}

Rob H.P. Hilgers, Paul M.H. Schiffers, Wendy M. Aattsen, Gregorio E. Fazzi,

Jos F.M. Smits, Jo G.R. De Mey.

Department of Pharmacology \& Toxicology, Cardiovascular Research Institute Maastricht (CARM), Universiteit Maastricht, the Netherlands.

Based on: Arterioscler Thromb Vasc Biol. 2004;892-897 


\section{Abstract}

Objective. To test whether membrane-bound angiotensin-converting enzyme (tACE) is involved in arterial temodeling, we applied unilateral carotid artery ligation and studied utrine arteries before, during and after pregnancy in $\mathrm{t}-\mathrm{ACE} / \%$ and $\mathrm{t}-\mathrm{ACE}+/ 4$ mice.

Results. In carotid arteries of $\mathrm{t}-\mathrm{ACE}$ mice, blood pressure, onter diameter and medial cross-sectional area ( $\mathrm{mCSA}$ ) wrere reduced, while blood flow and the number of medial cells wete not modified. In the ligated carotid attery, mCSA and the number of medial cells were increased while outer D and distensibility were reduced. These changes were significantly less pronounced in $\mathrm{t}-\mathrm{ACE} /$ than $\mathrm{t}-\mathrm{ACE} / *$ mice. In the contralateral carotid artery, blood flow and outer diameter were comparably increased in the absence of significant alterations of mCSA or the number of medial cells in both strains.

In uterine arteries of $\mathrm{t}-\mathrm{ACE} \%$ mice, internal diameter was larger and $\mathrm{mCSA}$ was unaltered. At term pregnancy, internal diameter and mCSA of the uterine antery were reversibly increased. Structural changes of the uterine artery during and after pregnancy were comparable in both strains.

Conclusion. Tissue-ACE contribures to arterial structure and remodeling. It plays a major role in hyperplastic inward remodeling of the carotid artery imposed by blood flow cessation, but is not essential for ourward hypertrophic and subsequent inward hypotrophic remodeling of the uterine artery during and after pregnancy. 


\section{Introduction}

Many components of the renin-angiotensin system (RAS) are localized in tissues indicating the existence of a local RAS.132,226 Angiotensin-converting enzyme (ACE) is present in all major organs and blood vessels.32 "This tissu bound ACE plays a major role in the local ptoduction of angiotensin II (Ang II) and in the local degradacion of bradykinin. 322

Ang II modulates vasomotor tone, ${ }^{8}$ cell growth and apoptosis, 1428 cell migration and extracellular matrix deposirion, 241,33 and stimulates the production of other vasoconstrictot- and growth Eactors. 22.95 It plays important roles in the functional and structural integrity of the arterial wall and in the pathogenesis of cardiovascular diseases 1478 Experimental and clinical studies with ACE inhibitors and angiotensin type $1\left(A T_{1}\right)$ teceptor antagonists consistently documented beneficial effects of these agents in treating and prevenung cardiovascular diseases such as hypertension and atherosclerosis. ${ }^{36,423}$, 48 Some of the beneficial effects of $\mathrm{ACE}$ inhibitors can be atributed to reduced breakdown of bradykinin. Antiproliferative effects of kinins via $B_{2}-1$ recoptors were demonstrated in atretial injury models using both ACE inhibitors and B-feceptor antagonists. $138,437,528$

Pressure and flow determine circumferential wall stress (CWS) and wall shear stress (WSS) in the arterial system. On an acute basis they modultate arterial smooth muscle tone: an increase in transmutal pressure triggers a myogenic contractile response: ${ }^{270}$ an increase in flow induces an endothelium-dependent wasodilatation. 50 On a chronic basis, negative feedback controll of CWS and WSS is achieved by modulation of arterial wall thickness and atrerial lumen diameter, respectively, 21,434 The mechanisms of this arterial remodeling largely remain to be established. 47,547,555 They might movolve Ang II that is produced within the atterial wall in response to an increase in CWS." Furthermote, there is incteasing evidence that elevated WSS releases bradykinin from the endothelium. ${ }^{37,223}$

Arterial remodeling in response to altered blood flow is often investigated using surgery to shunt flow between adjacent arteries. $122,173,282,203,300,434,46,3,4,5,547,555$ For instance, upon unilateral ligation of a catotid attery, the arterial blood flow is acutcly and persistently abolished in the ipsiateral artery and doubled in the contralateral artery. There fter the lumen diameter and wall mass of the wessels are modifed and neointima may develop. ${ }^{3 w}$ "This approach is increasingly used in the mouse to investigate the moleculat mechanisms that patticipate in the structural responses of large elastic arterics

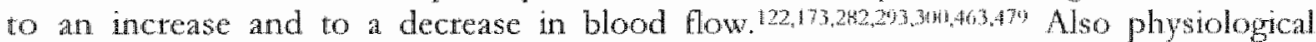
challenges may be considered. During pregnancy, blood flow through the uterine circulation increases substantially and reversibly. In the mouse, the uterine vasculature undergoes luminal expansion and an increase in wall mass, ${ }^{22}$ to accommodare this increase in uterine blood flow. The relationships between imposed, physiological and pachological arterial remodeling remain incompletely understood. Furthermote, whilc structural consequences of increased transmural pressure differ between large clastic and small muscular arteries, ${ }^{9,37,439}$ regional heterogeneity of flow-related atterial remodeling has been lintle investigated.

Two strains of ACE deficient mice have been genented, one that completely lacks somatic ACE ${ }^{1+10}$ and one that lacks the carboxy-terminal membrate-spanning anchor 
region. ${ }^{141}$ In the latter tissue $A C E$ deficient ( $t-A C E \%$ ) mice, blood pressure is reduced ${ }^{2}$.4! and the structural and mechanical properties of the aorta, catotid antery and mesenteric resistance artery, are modified ${ }^{2}$

In the present study, we lyypothesized that membrane-bound $A C E$ plays a pivotal role during flow-induced arterial remodeling. To test this hypothesis we used two models of flow-related arterial remodeling. We applied unilateral carotid artery ligations 300 and studied uterinc arteries before, during and after pregnancy ${ }^{223}$ in wild-type and $\mathrm{t}-\mathrm{ACE}$. mice.

\section{Methods}

\section{Aniwnals}

The generation of mice with C57Bl6/SV genetic background and lacking ussue-bound angiotensin converting enzyme ( $\mathrm{t}-\mathrm{ACE}$ ) has been described by Esther and colleagues. ${ }^{4}$ Mice heterozygous for the mutated ACE allele ( $t$-ACE $/$ ) were mated to obtain mice

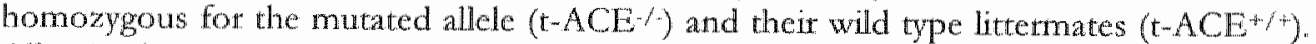
All animals were housed in groups of 2 to 4 and had free access to standard food (SRMA1210; Hope Farms, Woerden, the Netherlands) and tap water. All experiments were conducted according to institutional guidelines. Genotyping was perfomed as previously described ${ }^{2}$

\section{Bload Presitre and Heart Rate}

Male mice were anaesthetised with pentobarbital $(10 \mathrm{mg} / \mathrm{kg})$ and fixed on a heating-pad to control body temperature at $37^{\circ} \mathrm{C}$. A saline filled catheter (PE 25) was inserted into the abdominal aorta via the femolal artery and connected to a pressure transducet (Microswitch, model 156PC 156 WL, Honeywell Inc. Amsterdam, the Netherlands). Blood ptessure signals were recotded and 10 minutes of stable blood pressure recordings were used to calculate the mean arterial blood pressure and heart rate.

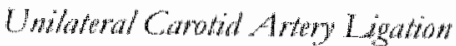

At 3 to 4 months of age, male $t-A C E+/ t(n=12)$ and $t-A C E / \quad(n=12)$ mice were anaesthetised with ketamine and xylazine $(100$ and $10 \mathrm{mg} / \mathrm{kg}$ SC). Body temperature was mantained ar $37^{\circ} \mathrm{C}$ by a themostatically controlled heating platform. Both common carotid arteries were exposed through a midline incision in the neck. In half of all the animals (experimental animals), the left common carotid artery was ligated with 5.0 surgical suture at the carotid bifurcation as described by Kumar and Lindner. ${ }^{300}$ One of the $\mathrm{t}-\mathrm{ACE}+\mathrm{E}^{+}$and wo of the $\mathrm{t}-\mathrm{ACl}$ - mice died within 24 hours after the surgery. In sham-operated $t-A C E^{*} /$ and $1-A C E /$ mice, right and lef carotid arteries were exposed but not ligated. The animals were allowed to recover for 4 weeks atter sham sutgery or unilateral carotid artery ligation.

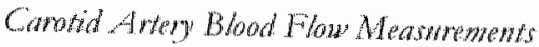

Four weks after inital surgery the animals were again anesthetized with ketamine and xylarine. Blood flow in the left and right carotid artery was recorded for $\left[-A C E^{+/ 4}\right.$ and $t$ 
$\triangle \mathrm{CE}$ - mice that had undergone either sham surgery or unilateral carotid artery ligation. We used a transit-time ultrasonic flow probe ( $0.5 \mathrm{~mm} V$ series, Transonic Systems) that was mounted on a micromanipulator and positioned halfwy between the aortic arch and the carotid artery bifurcation. Mean blood flow was obtained by averaging during a 5 minute period for the left and right carotid artery of each animal." 7 ?

\section{Pregrang-Induad Uterine Arew Remadeling}

Female mice (age 4 to 6 months) were used to study uterine arterial remodeling, comparing $t-A C E-/$ with $t-A C E^{t / t}$. In wiew of the infertility of male $t-A C E-$ mice, ${ }^{\text {th }}$ male $t-A C E^{+/+}$mice were used for mating virgin (in the remainder of this article referred to as nonpregnant) $t-A C E^{* /+}$ and $t-A C E-\%$ mice. Consequendy, fetuses grown in $t-A \mathrm{CE}^{-/}$ mothers had the $t-A C E^{+/}$genotype, and fetuses grown in $t-A C E^{+/ t}$ mothers had the $t-$ ACE ${ }^{+/}$genotype. Uterine arteries were investigared before pregnancy (NP), at late pregnancy (LP; day 18-19) and at 7 days postpartum (PP).

\section{Pressute-Diameter Capwes}

Catotid artery segments $(5 \mathrm{~mm}$ long, halfway between the aortic atch and the carotid bifurcation) and uterine artery segments ( $3 \mathrm{~mm}$ long, halfway between the ovaries and the vagina) were isolated and transferred into an organ chamber (arteriograph system, Living System Instrumentation, Burlington, VT, USA). The chamber was filled with calcium-free HEPES buffer containing $10 \mu \mathrm{mol} / \mathrm{L}$ sodium nirroprusside to assure maximal vasodilatation. Due to the intransparancy of the carotid artery, outer diameters were recorded in the carotid artery, and internal diameters for the uterine artery. A pressute. diameter relationship was established by reconding the diameter during stepwise (10 mmFg) increases in intraluminal pressures from 20 to $150 \mathrm{~mm} / \mathrm{Hg}$. After each step the vessel was allowed to equilibtate until a stable diameter was reached. After the experiment, the arteries were fixed at a transmural pressure of $100 \mathrm{~mm} / \mathrm{Hg}$ in $4 \%$ phosphate-buffered formaldehyde and stored in $70 \%$ ethanol.

\section{Morplowery}

rixed vessels were embedded in paraffin and cross-sections (4 $\mu$ m) were stained with Luwson"s solution (Boom, Meppel, the Netherlands) to visualize the internal and external clastic laminae. In the case of carotid arteries the morphometric analysis focussed on the central part of the vessel, halfway between the aortic atch and the carotid bifurcation. Unlike the distal part ${ }^{300}$ near the ligation, the central patt showed no signs of neointima Fomation of inflammation. Video images were made from cross-sections using a Zeiss axioscope and a standard charge-coupled device camera (Sony). Using J AVA sofrware (Sigma Scan, Jandel Scientific, Corte Madera, CA, USA) the circumference of the internal and external elastic laminae wene detemined. From these values the media cross-sectional area (CSA) was calculated. The average number of medial nuclear profilcs per cross section was measured by counting the nuclear profiles in the smooth muscle layers of hematoxylin-cosine stained cross-sections ( $n=3$ for each artery and individual mouse). Elastin and collagen were determined in carotid arteries, but not in uterine arteries because of the small size of these vessels. Digital images of Lawson stained cross-sections were made at $400 \mathrm{X}$ maginification. Using Leica QWin software the density of elastin was 
detemined in two areas of the media and avetaged. Elastin content was calculated by multiplying elastin density with the medial CSA. To stain collagen, cross sections were deparafinised and incubate in phosphomolybdenic acid $(0.2 \%)$ for 5 minutes, followed by incubation with Sirius Red $(0.1 \%$ in saturated picric acid solution for 90 minutes. After washing with $0.1 \mathrm{M} \mathrm{HCl}$ for 2 minutes, sections were dehydrated and protected with coversips. ${ }^{28}$ The densiry and content of collagen in the media were quantifed as described for elastin.

\section{Pbyriologian Solutions and Drags}

The composition of the $\mathrm{Ca}^{2+}$-free $\mathrm{HBSS}$ was (in mol/ L) $144 \mathrm{NaCl} 4.7 \mathrm{KCl}, 1.2 \mathrm{MgSO}$, $1.2 \mathrm{KH}_{2} \mathrm{PO}_{4}, 14.9 \mathrm{HEPES}$ and $5.5 \mathrm{glucose}$. The drugs used were pentobarbital sodium Nembutal, CEVA SANTE Animale BV, Massluis, the Netherlands), ketamine, xylazine, and sodium nitroprusside (Sigma Chemical, St Louis, MO).

\section{Statistics}

Results are expressed as means $\pm S E M$. In all experiments, equals the number of mice and vessels. Statistical significance of differences between sets of data was determined by ANOVA with post-hoc Student-Newman-Keuls test. A $P$-value of $<0.05$ was considered to indicate a significant difference.

\section{Results}

\section{General Ob̆sentrom}

Blood pressure was significantly lower in male $t-A C E-/$ mice compared to $t-A C E^{+/ 4}$ mice $(75 \pm 4 \mathrm{mmHg}$ versus $100 \pm 5 \mathrm{mmJg})$, while heart rate was comparable $(627 \pm 21$ bpm versus $662 \pm 12$ bpm, respectively). Carotid atterial blood flow (meath of left and right carotid attery) did not differ significantly between $\mathrm{t}-\mathrm{ACE} / \mathrm{and}-\mathrm{ACE} /+(0.39 \pm 0.04$ $\mathrm{mL} / \mathrm{min}$ versus $0.40 \pm 0.02 \mathrm{~mL} / \mathrm{min}$, respectively). In both groups of mice, left carotid artery ligation resulted in an elimination of blood flow in the occluded vessel and in a comparable substantial incrase of blood flow in the right catotid artery $(0.81 \pm 0.11$ $\mathrm{mL} / \mathrm{min}$ in $\mathrm{t}-\mathrm{ACE} / \mathrm{and} 0.79 \pm 0.07 \mathrm{~mL} / \mathrm{min}$ in $\mathrm{t}-\mathrm{ACE}+1)$.

In female mice, deficiency of tissue $\mathrm{ACE}$ did not result in abnomalities during and after pregnancy, as far as bodyweight, fetal and placental weight and the number of fetuses and pups are concened (Table 7.1). However, cardiac hypertrophy during and after pregnancy was less pronounced in $-A C E$ mice compared to $-A C E$ - mice $^{+}$(Table 7.1 ).

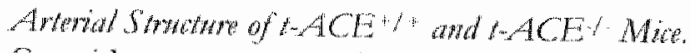

Catotid artery outer diameters were significandy smaller in $t$-ACE $/ \%$ compared to $\mathrm{ACE}$ t mice, especially at the highest intraluminal pressure $(615 \pm 14 \mu \mathrm{m}$ versus $675 \pm 13$ $\mu \mathrm{m}$, respectively, Figure $7.1 \mathrm{~A}$ ). Medial cross-sectional area (CSA) was significantly smaller in carotid artery of $\mathrm{ACE}$ mice $\left(14.1 \pm 0.810^{3} \mu \mathrm{m}^{2}\right)$ that $\left[-A C E /\right.$ mice $\left(20.4 \pm 0.910^{\circ}\right.$ $\left.\mu \mathrm{m}^{2}\right)$. The densigy and content of collagen did not differ, but the density and content of elastin tended to be smaller in carotid artery of $\mathrm{t}-\mathrm{ACE} /$ than $\mathrm{t}-\mathrm{ACE} \mathrm{E}^{+/}$mice (Table 7.2 ). 
In contrast to carotid arteries, uterine arteres of nonpregnant $-A C E^{-1}$ were significandy wider compared to $t-A C E^{-/ 4}$ mice $(290 \pm 10 \mu m$ versus $251 \pm 7 \mu m$, at $140 \mathrm{mmHg}$. Figute 7.1B), despite comparable media CSA (Figure 7.11 , insert).

\section{Carotid Antery Stmotwal Clonges in Response to Alared Blood Flow}

The hypenperfused right carotid artery, displayed a significant increase in outer diameter (Figure 7.2). At high transmural pressure the diameter was increased in both stans. At low transmural pressure the diameter was significanty increased in $\mathrm{T}-\mathrm{ACE}$ - but not $\mathrm{t}$ ACE ${ }^{+}$mice. Medial collagen, elastin and CSA ware not significantly altered in the right carotid antery of $t-A C E$ " and $t-A C E+/+$ mice after four weeks exposure to elewted blood low (Figure 7.3 and Table 7.2 ).

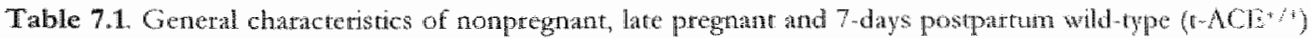
and dissue angiotensin-conveting enzyme deficient (t-ACE $)$ mice.

\begin{tabular}{|c|c|c|c|c|c|c|c|}
\hline mice & state & BW $(\mathrm{g})$ & $\mathrm{HW}$ (mg) & $\mathrm{FW}(\mathrm{mg})$ & $P W(m g)$ & th fornses & st pups \\
\hline \multirow[t]{3}{*}{$\mathrm{ACE}^{\mathrm{i} / \mathrm{T}}$} & $N P^{P}$ & $24 \pm 1$ & $125 \pm 6$ & & & & \\
\hline & LP & $38 \pm 1 *$ & $148 \pm 9$ & $765 \pm 91$ & $111 \pm 4$ & $8 \pm 1$ & \\
\hline & $\mathrm{PP}$ & $29 \pm 2 *$ & $162 \pm 7 *$ & & & & $5 \pm 1$ \\
\hline \multirow[t]{3}{*}{$\triangle \mathrm{ACE}$} & NP & $23 \pm 1$ & $113 \pm 7$ & & & & \\
\hline & I.P & $33 \pm 1$ * & $116 \pm 7+$ & $895 \pm 110$ & $99 \pm 4$ & $7 \pm 0$ & \\
\hline & $P P$ & $27 \pm 1 *$ & $141 \pm 9^{*}$ & & & & $6 \pm 1$ \\
\hline
\end{tabular}

Abbtevtations used: NP: nonpregnant; LP: late pregnant; PP: postpartum; BW: body weight; HW: heart weight; FW: ferus weight (withont placenta), PW: placental weight. All wet weights, *P<0.05 compared with NP mice, $\uparrow P<0.05$ compared with $t A C E+/ 4$. Walues are expressed as means $\pm S E M$.

A

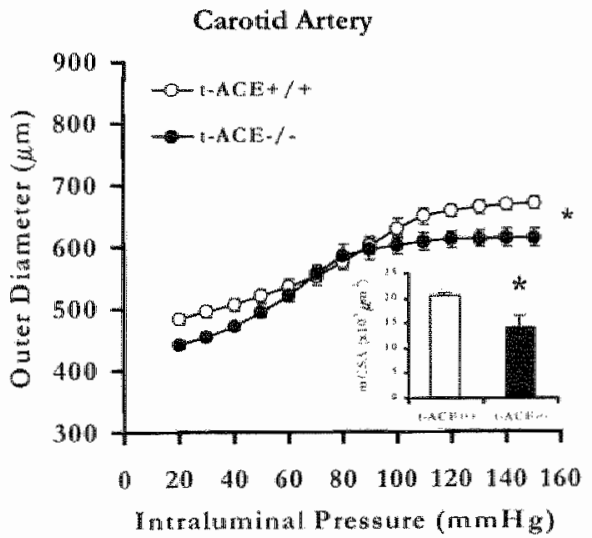

B

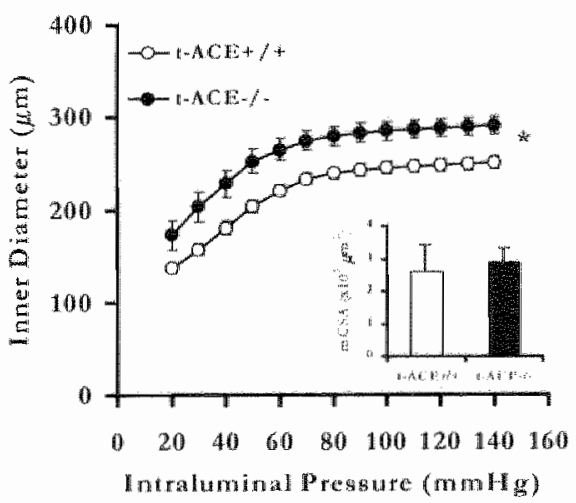

Figure 7.1. Relations between distending intraluminal pressure and outer diameter in isolated carotic arteries of

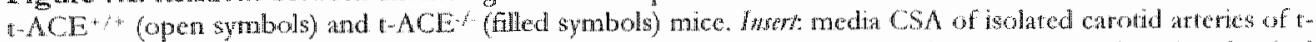
$\mathrm{ACE}^{*}$ (open bars) and $t-A C E^{-/}$(Gilled bars) male mice (A). Relations berween distending intraluminal pressure and innet diameter in isolated uterine arteries of nonpregnant $1-\mathrm{ACE}+/+$ (open symbols) and $\mathrm{C}-\mathrm{ACE}$ ? (Glled stmbols) mice. Intert. media CSA of isolated uterine arteries of nonpregnant $A C E$ " (open bars) and t ACE (filled bars) mice (B). Values ate meang $\pm S E M$. $p<0.05$ versis $\mathrm{t}-\mathrm{ACE}$ " 
A.

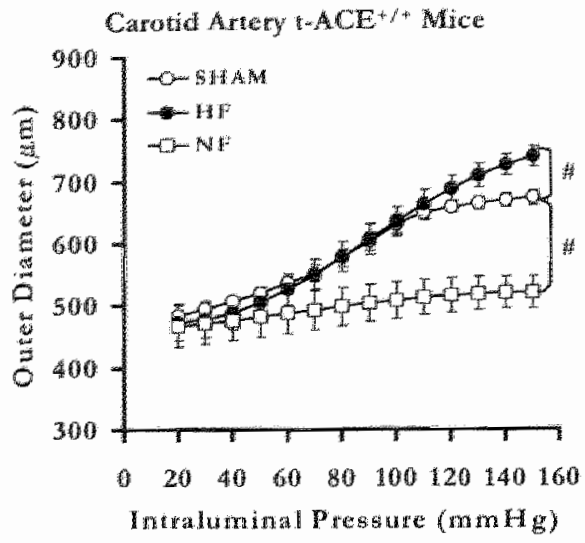

B

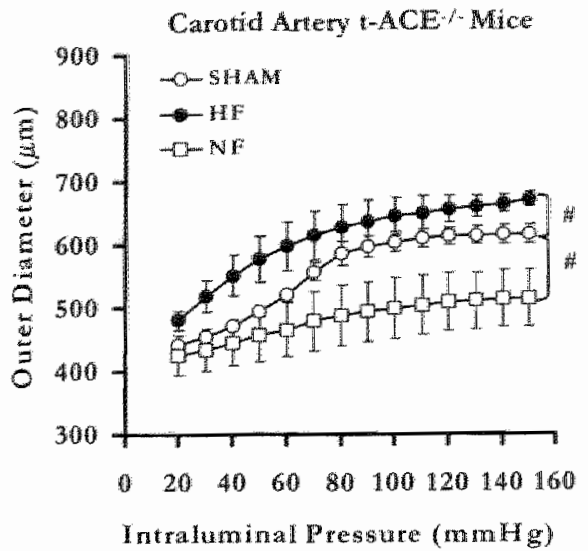

Figure 72 . Effects of unatateral carorid artery ligation on relations between distending intraluminal pressure

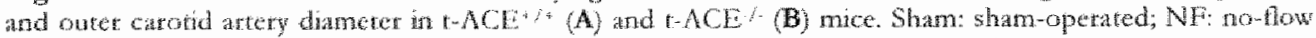
(iigated) wessels; HE: high flow (hyperperfused) vessels. Valucs are means \& SEM. \# P<0.05 w Sham.

Ligation of the left carotid artery resulted in a significant reduction of the outer diameter (Figure 7.2) accompanied by an increase in medial CSA (Figute 7.3) and a dramatic reduction in distensibility of the ligated vessel. The diameter reduction was more pronounced in $-A C E+1+$ mice (from $671 \pm 13$ to $516 \pm 25 \mu \mathrm{m} ; 23 \%$ reduction) than in $t$ ACE mice (from $625 \pm 16$ to $515 \pm 45 \mu \mathrm{m} ; 18 \%$ reduction). Also the medial hypertrophy

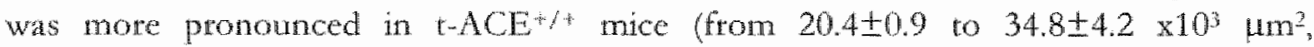
reptesenting a $71 \%$ inctease) than $\mathrm{ACE}$ mice (from $14.1 \pm 0.8$ to $18.1 \pm 2.3 \times 10^{3} \mu^{2}$, representing a $28 \%$ increase) and involved a large increase in the number of medial cells in $(-A C E+/+$ bur not $-A C E$ mice (Figure 7.3 lower panel).

In the media of the ligated left carotid artery of both $\mathrm{t}-\mathrm{ACE} / \cdots$ and $\mathrm{A}-\mathrm{ACE}+\mathrm{t}$ mice, staning for the monocyte/macrophage marker ED1 was rare and all cells stained for smooth muscle $\alpha$-actin (not shown). Collagen and elastin densities were not alteted in the ligated yessels, but due to the higher mediat mass in the ligated carotid attery of $\mathrm{t}-\mathrm{ACE}$ mice, clastin content was significantly incteased (Table 7.2).

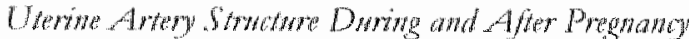

Duting latc ptegnancy maximal humen diameter of uterine arteries increased significanty in $\left[-\mathrm{ACB}^{+/}\right.$(from $251 \pm 7$ to $384 \pm 14 \mu \mathrm{m}$ ) and $\mathrm{t}-\mathrm{ACE} / \mathrm{mice}$ (from $290 \pm 10$ to $384 \pm 16$ Hin, Figure 7.4). Medial CSA increased significandy and comparably during pregnancy in UA of both $t-A C E \%$ (from $2.6 \pm 0.3$ to $5.2 \pm 0.8 \times 10^{3} \mu \mathrm{m}^{2}$ ) and $\mathrm{ACE}$ - mice (from $2.9 \pm 0.3$ to $5.1+0.4 \times 10^{3} \mu^{2}$, Figure 7.5 , upper panels). In neither strain was the medial hypertrophy accompanied by a significant change in the number of nuclear profiles per medial cross section (rigute 7.5, lower pands). By 7 days postpartum, most of the structural changes in the uterine artery were reversed (Figure 7.4 and 7.5 ). Maximal lumen diameter had patially regressed to pre-pregnant values in both $t-\mathrm{CE}^{+/+}$mice (from $384 \pm 14$ to $290 \pm 21 \mu \mathrm{m}$ ) and $\mathrm{t}-\mathrm{ACE}$ - mice (from $384 \pm 16$ to $301 \pm 17 \mu \mathrm{m}$ ). Medial CSA fagressed to nonpregnant values in both strains (Figure 7.5). 


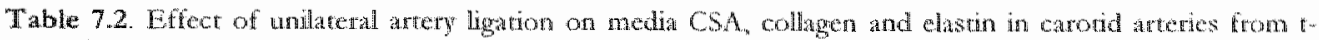
$\mathrm{ACE}+4$ and $\mathrm{CACE}$ - mice.

\begin{tabular}{|c|c|c|c|c|c|}
\hline & Strain & Truit & Normal How & High Flow & No Flow \\
\hline \multirow[t]{2}{*}{ dredila CSA } & $\mathrm{C}-\mathrm{ACE}^{+1+}$ & $100^{3} \mathrm{~mm}^{3}$ & $20.4 \pm 0.9$ & $19.4 \pm 0.8$ & $34.8 \pm 4.2$ \\
\hline & $-\mathrm{ACE}^{-3}$ & $101 \mathrm{~lm}^{2}$ & $141 \pm 0.8$ & $17.8 \pm 2.4$ & $18.1+2.3$ \\
\hline \multirow{4}{*}{ Collagen } & \multirow{2}{*}{$-\mathrm{COE}^{+/+}$} & $\%$ & $39.1+6.4$ & $33.3 \pm 8.2$ & $20.5 \pm 2.1$ \\
\hline & & $10^{3} \mu m^{2}$ & $7.3 \pm 113$ & $6.4 \pm 1.8$ & $6.9+1.8$ \\
\hline & \multirow{2}{*}{$\mathrm{TACF}$} & $\%$ & $46.7 \pm 7.1$ & $31.5 \pm 8.0$ & $32.8 \pm 3.8$ \\
\hline & & $10^{3} \mathrm{~km}^{2}$ & $7.2 \pm 1.0$ & $5.5 \pm 2.1$ & $6.8+1.9$ \\
\hline \multirow{4}{*}{ Eilastir } & \multirow{2}{*}{$t-\mathrm{ACE}^{+}$} & $\%$ & $37.4 \pm 15$ & $35.7 \pm 5.5$ & $31.3 \pm 5.4$ \\
\hline & & $10^{3} \mu \mathrm{m}^{2}$ & $6.0 \pm 0.6$ & $6.8=0.8$ & $10.0 \pm 1.0$ \\
\hline & \multirow{2}{*}{$-A C E$} & or & $31.3 \pm 4.9$ & $35.3 \pm 4.8$ & $27.7+3.11$ \\
\hline & & $10^{3} \mu \mathrm{m}^{2}$ & $4.9 \pm 0.5$ & $6.0 \pm 0.7$ & $5.4 \pm 0.7$ \\
\hline
\end{tabular}

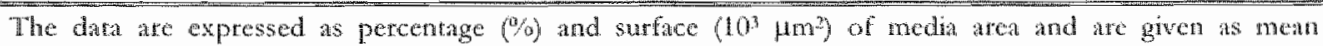
walues ISEM. * P 0.01 versus normal flow (sham-operated carotid artery) and high fow fright carotid artery).

A

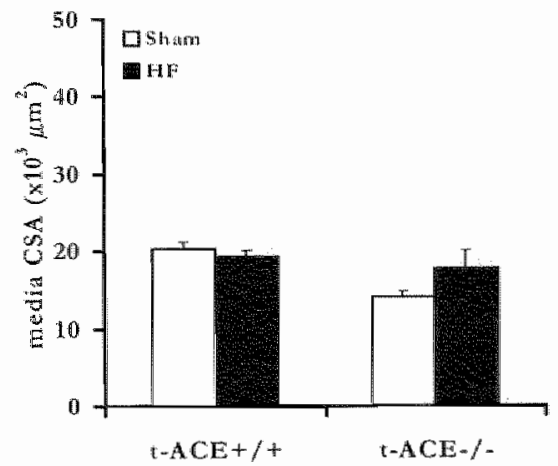

C

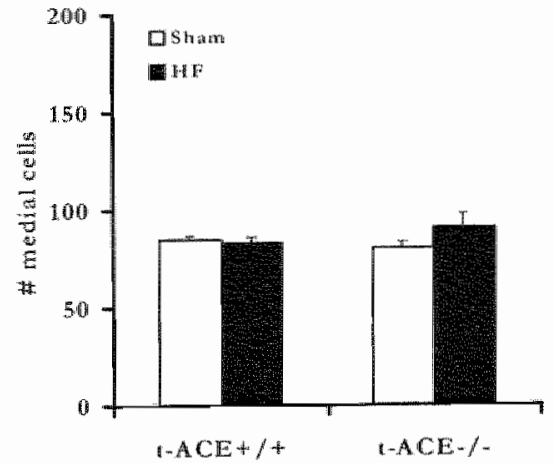

B

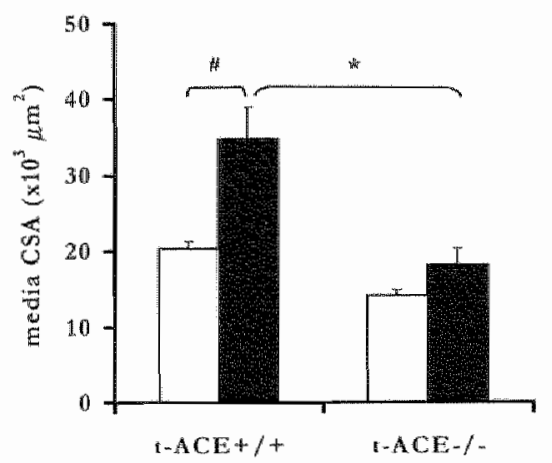

D

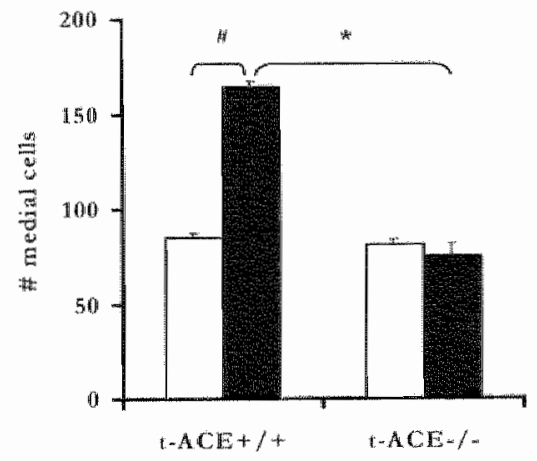

Figure 7.3. Eleces of unilateral carotid artery lagation on medial $\mathrm{CSA}$ (upper panel) and the number of medial cells per cross-section (lower pane) of byperperfused (A and $C$ ) and ligaled ( $B$ and $D$ ) carotid artentes in $t$

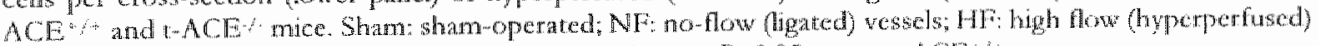

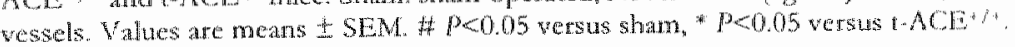


A

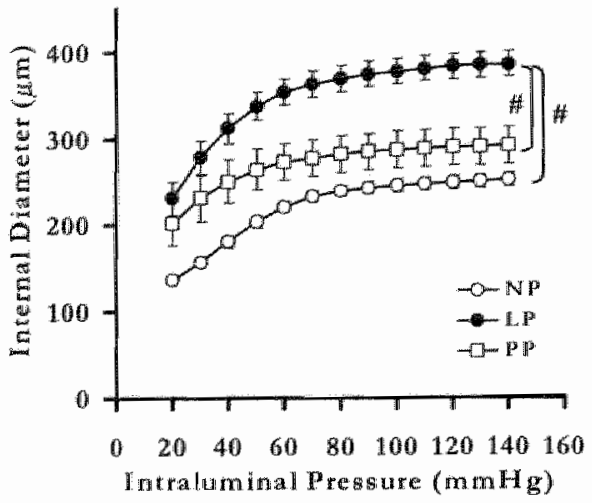

B Uterine Artery th-ACE $\%$ Mice

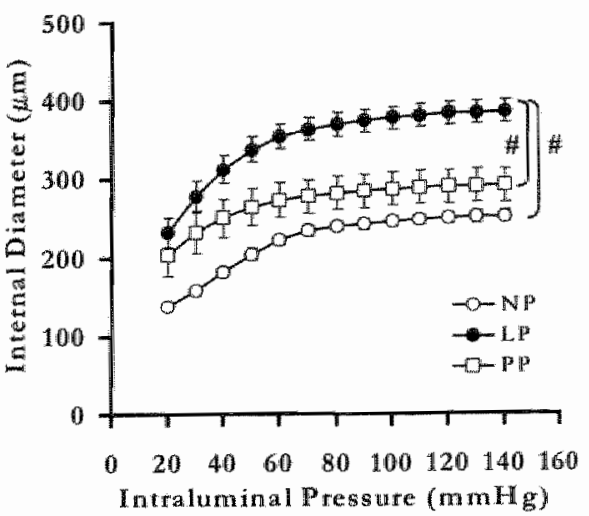

Figure 7.4. Effects of pregnancy on the thations berween distending intraluminal pressure and inner diameter

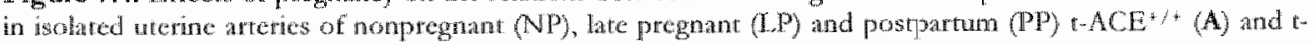
ACE (B) mice. $\mathbb{P}<0,05$ versu $N P$ and $P P$. Values are means \pm SEM.

\section{Discussion}

According to out experimental findings, membrane-bound ACE participates in the control of arterial structure. The tole of the enzyme differs between imposed and physiological atterial structural changes.

In line wh the pressor and hypertophic effects of angiotensin $I I, 4104,49 \%$ t $A C E$ deficient mice displayed lower blood pressure, teduced carotid artery medial mass and reduced pregnancy-related cardiac hypertophy. Furthemore, as previously reponted, ${ }^{2}$ the carotid artery was nartower but more distensible despite unaltered carotid atterial blood Fow. Mantenance of carotid artery blood flow despite reduced blood pressure implies reduced cerebrovascular resistance. This is latgely due to blunted vasomotor tone and an increasco structural diamerer of resistance arteries as observed in the mesenteric, ${ }^{2}$ and wrene arterial bed (this study). We previously observed that chronic phamacological ACF inhibition in wild-type mice resulted in comparable alreations of carotid artery structure and mechanics. ${ }^{2}$ Combined, these observations indicate that $t-A C E$ plays a role in the establishment and mantenance of the balance between local hemodynamic and mechnical tonces, on the one hand, and the structural and mechanical properties of large clastic arteries, on the other hand. This tole of $\mathrm{ACE}$ is largely similar in male and female mice. ${ }^{2}$ Direct trophic and indirect, $e \cdot g$ pressure-talated, influences of angiotensin II or bradykinin, may be involved. Because a 2 -week treatment with a $B_{2}$ receptor antagonist blunts the difference in catotid artery mass and distensibility, but not the difference in blood pressure between $\left[-A C E\right.$ - and $t-A C E^{4 /+}$ mice, ${ }^{2}$ ptimarily bradykinin seems to be involved. 
A

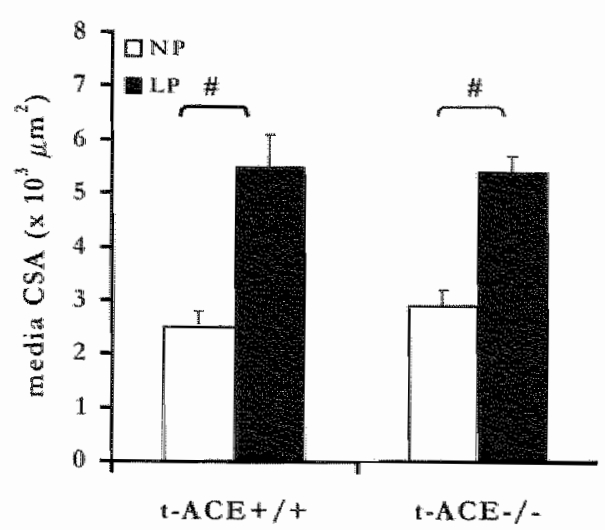

C

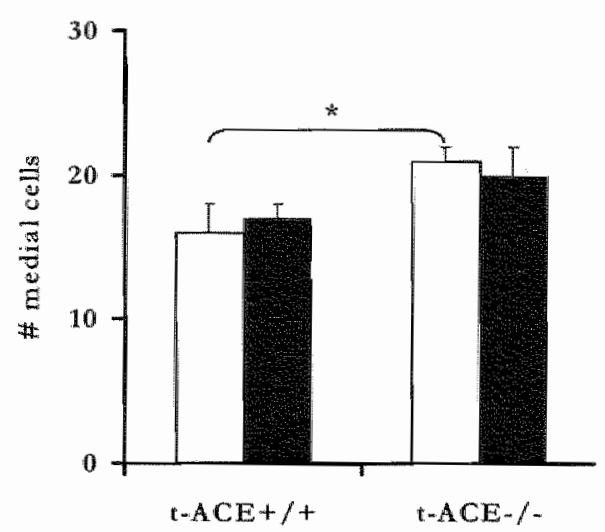

$\mathbf{B}$

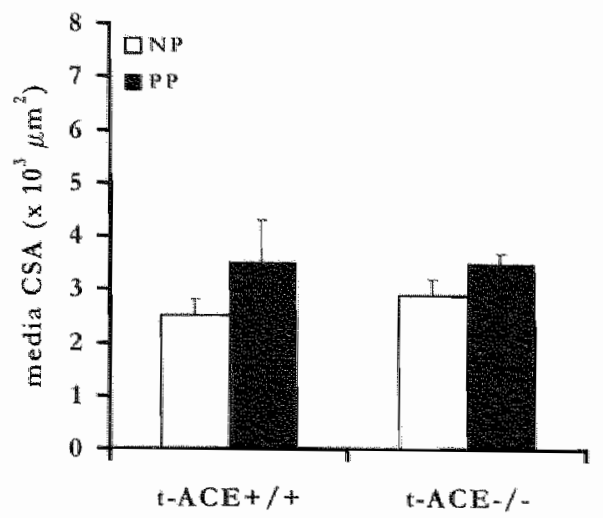

$\mathrm{D}$

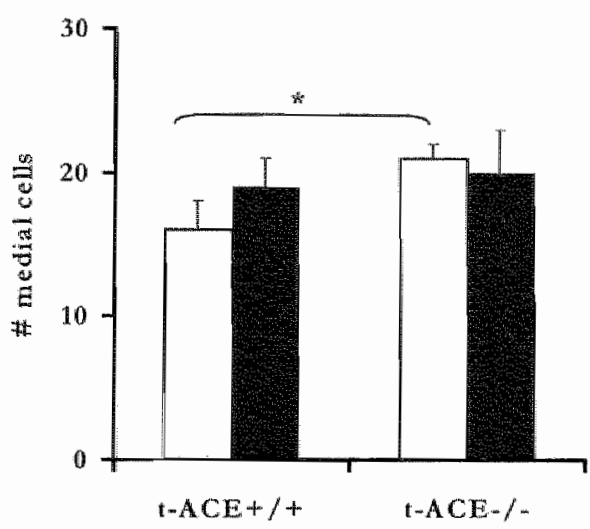

Figure 7.5. EEfeets of pregnancy on medial CSA (upper panel) and the mumber of medial cells per cross-

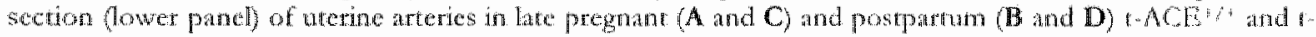

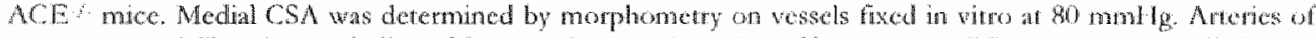
monpegnant (NP) micc are indicated by open bars, and areries of hate pregane ( $\mathrm{P}$ ) of postpartum (PP) mice

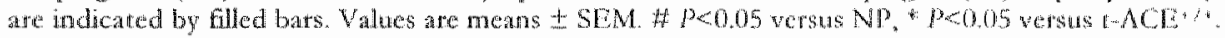

In contrast to large elastic arteries, but similar to small muscular mesenteric arteries, uterine arteries of $t-A C E$, displayed a larger lumen diameter despite a comparable medial surface area. This regionality is reminiscent of the hypettophy and inward eutrophic remodeling that was described for large elastic and small muscular arterics in bypertension, respectively ${ }^{377,39}$ The molecular and stuctural basis of this tegional heterogeneiry is langely unknown. It was proposed that negative feedback control of local mecharical forces can be achieved to a larger extent in muscular arteries than in clastic atteries by altetations in vasomotot tone, i.e. by myogenic tone and flow-induced wasodilatation. $856,157,270,507$

Local mechanical forces that govern arteral structure include circumferential wall stress (CWS) and wall shear stress (WSS). 270,47 Chronic changes of these forces result in 
arterial remodeling. $122,173,223,222,293,304,434,46,47,54,55,3$ These arterial adaptive structural responses involve partly interrelated aterations in (a) arterial wall mass, (b) mechanical propertics of the arterial wall material and (c) arterial hmen diameter. Because CWS and WSS can stimulate intra-arterial production of angiotensin II and bradykinin, tespectively, $21,3,23$, we compared arterial remodeling in large and small muscular arteries of $\mathrm{A}-\mathrm{ACE}$ and $t-A C E+/ 4 i c e$. In the mouse carotid artery ligation model no differences have been observed between males and fenales. ${ }^{253}$

The increase in contralateral blood flow after unilareral carotid artery ligation, was comparable in $\mathrm{t}-\mathrm{ACE} / \mathrm{H}$ and $-\mathrm{ACE}+1+$ mice. Following 4-weeks exposure to doubled blood flow, arterial diameter was increased while arterial wall mass and collagen and elastin content were nor allered. Although $\mathrm{t}-\mathrm{ACE}$ was not essential for this outward arterial remodeling, distinct ultrastructural altenations might have contributed to the expansion of the vessel. In the absence of $t-A C E$ which has been shown to be a deteminant of large artery stiffness, ${ }^{2}$ not only the maximal diameter but also the diameters at intermediate pressures were increased.

More marked differences were observed between ligated arteries of t-ACE $/$ and $\mathrm{t}$ $A C E^{-t}$. In ligated catotid arteries of $t-A C^{+/+}$mice, outer diameter and arterial distensibiliry were reduced while medial cross secuonal area and medial cell number were increased. The marked stiffening of the arterial wall proceeded without a change in collagen and clastin density; rather the elastin content significantly increased. Changes in diameter, medial cross sectional area and -cell number were less pronounced in $\mathrm{t}-\mathrm{ACE}$ than $\mathrm{t}-\mathrm{ACE} \mathrm{H}^{+/}$mice. These findings are in line with previously reported angiotensin IIinduced stimulation of vascular smooth muscle cell proliferation and fibronectin production $^{31,104,-41}$ They do, however, raise guestions about the stimuli for intra-arterial production of mediators. CWS, a teported scimulus for the local RAS, 21 is likely to drop in the ligated artery as a result of reduced flow-induced vasodilatation and proceeding wall thickening. WSS, a stimulator of the production of the other major ACE substrate bradykinin ${ }^{3725}$ and one of its major effectors nitric oxide ${ }^{69}$ is at least initially markedly reduced in the ligated artery. Despite these unknowns, $t-A C E$ seems to be involved in the structutal responec of large clastic arteries to a moderate increase and a marked decrease in blood flow.

In both $t-A C E$ and $-A C E+/$ mice, the increase in uterine blood flow during pregnancy was sufficient to support the survival and growth of a comparable number of fetuses. Diameter and medial mass of the uterine artery increased substantially during pregnancy possibly as an adaptation to the increasing WSS and CWS. In t-ACE mice, the diameter increase was somewhat smaller than in $-A C E+$ mice. We recently reported that outwat remodeling of the uterine artery during pregnancy is not altered in tissue kallikrein-deficient mice. ${ }^{223}$ Consequently, angiotensin II rather than bradykinin seems to be involved in the widening of the vessel. We can, however, not fully exclude (i) that uterine atterial blood flow increased to a lesser extent in pregnant $t-A C E-\%$ than $t-A C E+/ 4$ mice, and (ii) that less remodeling of the ressel was required, because it was already relatively expanded before pregnancy. In contrast to the diameter, the increase in medrat mass was comparable in $[-A C E /$ and $[-A C F+/$ mice. Because the number of nuclear profides per medial section did not change, the wall hypertrophy is primarily due to medial 
cell growth. Unlike for the hyperplasia in the no-flow carotid artery, $\mathrm{t}$-ACE $/$ is not essential for cellular hypertrophy in the uterine artery.

By 7 days postpartum, uterine artery medial surface area and diameter at mean arterial pressure did no longer differ from pre-pregnancy values. This illustrates the dynamic and plastic nature of the vascular bed. The reversal of the remodeling was obtained by a reduction of medial cell size. We previously reported that also tissuekallikrein deficiency did not impair reversal of uterine artery remodeling. ${ }^{223}$ Consequently, intramural production of neither angiotensin II nor bradykinin seem to be essential for the adjustments of urerine arterial wall mass and lumen diameter to the marked reduction in blood flow.

Not only the role of $\mathrm{t}-\mathrm{ACE}$ but also the nature of the remodeling differed in carotid and uterine arteries. Hyperperfusion led to expansion of both vessels but this involved wall and cellular hypertrophy in the uterine artery and not in the carotid artery. Hypoperfusion resulted in a reduction of lumen diameter in both vessels. This was accompanied by wall hypertrophy and hyperplasia in the carotid artery while the number of medial cells was nor modified and their size probably reduced in the uterine attery. These regional differences seem not only due to the different extents and kinetics of the imposed and physiological blood flow changes. In tat and mouse small muscular mesenteric arteries, a doubling of blood. flow leads to outward hypertrophic remodeling and a marked reduction of blood flow leads to inward hypotrophic remodeling. $71,4.4,555$ Unlike in the uterine artery, these two types of mesenteric artery remodeling are accompanied by medial cell proliferation and apoptosis ${ }^{71}$ leading to a gain and a loss of smooth muscle cells in the high flow and the low flow arteries, respectively. Whether the role of $t-A C E$ is more prominent in the remodeling of large elastic than in small muscular arteries, or more prominent in hyperplastic than in hypertrophic remodeling, awaits further experimentation.

The tegional heterogeneity and diversity of the role of $\mathrm{t}-\mathrm{ACE}$ in arterial structural changes, which we observed in an elastic artery and a pre-existing collateral artery such as the uterine artery, are in line with earlier findings. Angiotensin II and bradykinin stimulate and inhibit the proliferation of medial and neointimal smooth muscle cells in carotid arteries, respectively. ${ }^{104}, 13 \%, 528$ Yet, angiotensin 11 can promote shunaing of blood how through pre-existing collateral channels $s^{\prime}$ and has been reported to promote and inhibit angiogensis at low and high concentrations, respectively. ${ }^{5 / 6}$ The former mechanism, along with the proangiogenic effects of bradykinin ${ }^{5} 11$ seems to account for the beneficial cffects on capillary density and blood flow observed with intibitors of $\mathrm{t}-\mathrm{ACE}$ such as quinaprilate in peripheral and coronary ischemia. ${ }^{42}$

Despite its roles in the morphogenesis and remodeling of vascular beds and in spermatogenesis, ${ }^{1+15} \mathrm{t}-\mathrm{ACE}$ (this study) and even both membrane-bound and circulating $\mathrm{ACE}{ }^{35 \%}$ are not essential for optimal pregnancy outcome in the mouse. The various maternal systemic and local vascular alterations, including extensive temodeling and angiogenesis in the uterine vascular bed, can proceed in the absence of ACE-derived angiotensin $I I$ and do not require local production of bradykinin.2.2. Alternative biosynthetic pathways and various other growth factors and angiogenic stimuli might be upregulated during this physiological response on which there is considerable biological pressure. Recent genetic observations indicate that ACE might rather be detrimental 
during human pregnancy. ${ }^{35 \%}$ The $A C E \mathbb{I} D$ polymorphism affects besides $A C E$ activity, uteroplacental and umbilical blood flows and the recurrence of an adverse pregnancy outcome, including intrauterine growth retardation, in women with a history of ptecclampsia.

In summary, observations in $\mathrm{t}-\mathrm{ACE} /$ mice indicate that membrane-bound $\mathrm{ACE}$ plays different roles in the development and maintenance of the structural and mechanical propertics of large elastic and small muscular arteries. Moreover, our present findings in tACE $\%$ mice, combined with our eatlier findings in tissue kallikrein-deficient mice, ${ }^{23}$ indicate that intra-arterial production of angiotensin II contributes substantially to the hyperplastic remodeling of the ligated carotid artery but not to the hypertrophic and hypotrophic remodeling of the uterine artery during and after pregnancy. 
Chapter 8

\section{Uterine Artery Dilatation and Remodeling During Postponed Murine Pregnancy}

Rob H.P. Hilgers, Paul M.H. Schiffers, Gregorio E. Fazzi, Jo G.R. De Mey.

Department of Pharmacology \& Toxicology, Cardiovascular Research Institute Maastricht (CARM), Universiteit Maastricht, the Netherlands. 


\section{Abstract}

Objective. Aging was reported to reduce endothelium-dependent vasodilatation and outward arterial remodeling. We hypothesize that this (1) also applies to small muscular atterics in the uterine vascular bed and (2) adversely affects reproductive performance. We therefore monitored uterine artery vasomotor responses, structural changes and fetal number before and at the 18th day of first pregnancy in approximately 17 (young) and 58 (middlle age) weeks old mice.

Results. Uterine arteries were larger, but norepinephrine-induced constriction and dilatation in response to flow, acetylcholine, and $\mathrm{Na}$-nitroprusside were comparable in middle age compared to young mice. $\mathrm{N}^{(\omega)}$-nitro-L-arginine (1) reduced dilator responses to acetylcholine in both young and middle age mice, but (2) reduced flow-induced dilatation in young, but not middle age mice. Pregnancy had minimal effects on uterine arterial vasomotor responses in young mice, but enhanced myogenic tone in middle age mice. At term pregnancy, the increase in internal diameter, and in media cross-sectional area and the number of viable fetuses were significantly smaller in middle age than young mice.

Conclusion. Aging modifies shear stress-induced vasodilatation and blunts pregnancy-telated remodeling of the uterine artery. This might contribute to fetal growth retardation and mortality during postponed pregnancy. 


\section{Introduction}

Many epidemiologic and animal studies have shown that a sub-optimal fetal microenvironment can have persistent consequences for health in adulthood. Intra-uterine growth restriction, either via malnutrition or placental insufficiency, is associated with reduced birth weight and increased risk for metabolic and cardiovascular disorders in the adult life, 23,424

During mammalian pregnancy uterine blood flow increases dramarically ${ }^{\text {thi }}$ Adaptation of the uterine vascular bed to meet the incteasing metabolic demands of the growing fetus(es) occurs through vasodilatation, ${ }^{337}$ ourward remodeling and the development of new vessels (angiogenesis). ${ }^{\text {(i) }}$ ? These changes depend on the inner endothelial lining of the arterial bed. ${ }^{312}$

Currently, many women postpone childbearing for socio-economic reasons. Increasing maternal age (>35 years) at first childbirth is an independent risk factor for low birth weight and spontaneous abortion. ${ }^{163,399}$ In rodents such as mice, the number of inplanted fetuses is initially comparable between young and middle age anmals. However, a large number of fetuses dies during the third trimester of pregnancy when the increase in uterine blood flow should be most pronounced. ${ }^{561}$

Aging is an important risk factor for cardiovascular diseases such as ischemic heart disease. Although the mechanisms associated with vascular aging are multi-factorial, one underlying cause may be an age-associated endothelial dysfunction ${ }^{3.15}$ leading to (i) reduced bio-availibility of endothelium-derived vasodilators 258 (ii) impaired flow-induced vasodilatation, ${ }^{181}$ and (iii) a diminished atterial lumenal expansion and increased smooth muscle cell hypertrophy in response to altered blood flow. ${ }^{361}$

Most aging-related research concentrates on $>65$ years of age in humans or comparable stages of life in experimental animals. It is not clear whether significant vascular changes develop in important vascular beds between young adulthood and the mid-life stage. We hypothesize that flow-induced vasodilatation and arterial remodeling are particularly sensitive to aging and that this contribures to reduced reproductive performance and increased pregnancy-associated complications during postponed pregmancy. We therefore compared for 17 and 58 weeks old mice (i) vasomotor tesponses of the isolated uterine artery, (ii) pregnancy-induced uterine arterial changes, and (iii) fetal properties at 0.9 gestation.

\section{Materials and Methods}

\section{Animols}

17 weeks old (further referred to as young mice) and 58 weeks old (further referred to as middle age mice) $\mathrm{C} 57 \mathrm{Bl} / 6 \mathrm{~J}$ mice with a reported mean life span of 120 weeks ${ }^{\text {it }}$ were putchased from Charles River, Maastricht, the Nerherlands. All animals had free access to food and water. Pregnancy was achieved by mating with an experienced male of the same strain. The recovery of a vaginal sperm plug was considered to correspond to day 1 of pregnancy. At the $18^{\text {th }}$ day of their first pregnancy ( 0.9 gestation) young and middle age 
mice wete sacrificed. A group of age-matched virgin mice served as nonpregnant controls. Al experiments were conducted according to institutional guidelines.

\section{Tiswa Preparation}

Mice were sactificed with an overdose of pentobarbital (i.p.). Reproductive parameters (litter size and wable retuses) wete recorded. Viable fecuses were recognized by their pink appearance and their movement in response to a mechanical stimulus. Isolation of the main uterne artery was performed as described. 223

\section{Prosura Mugraph Experiments}

At the beginning of each experiment, the uterine artery segment was incubated for 45 minutes at $80 \mathrm{~mm}$ - In almost all vessels a basal constriction developed. The integrity of the endothelium was cvaluated by assessing the response to acetylcholine ( $1 \mu \mathrm{mol} / \mathrm{L}$ ) duting phenylephrine (1 $\mu \mathrm{mol} / \mathrm{L}$ )-induced constriction. Arteries that dilated $<60 \%$ of passive diameter (see below) were excluded. Puessure-induced (myogenic) tone was determined by subminting the arteries to $10 \mathrm{mmHg}$ pressure increments from 20 to 100 $\mathrm{mmHg}$ at 5 min interval in the absence of intraluminal flow. Percentage myogenic tone $(\% \mathrm{MT})$ at an intraluminal pressure of $80 \mathrm{mmHg}$ was calculated from the formula:

$$
\% \mathrm{MT}=\frac{\left(D_{\text {passine }}-D_{\text {untive }}\right)}{D_{\text {prassine }}} \cdot 1.00
$$

The passive diamerer is the diamerer obtained in Ca ${ }^{2+}$-free physiological salt solution containing sodium nitroprusside $(10 \mu \mathrm{mol} / \mathrm{L})$ which abolishes smooth muscle tone.

Afrer washing, a myogenic constriction returned. A concentration-response curve (CRC) with norepinephrine (on top of this myogenic tone) was constructed by cumulative exraluminal administration of norepinephrine ( $\mathrm{NE}, 0.01-10 \mu \mathrm{M}$ ) to the bath solution. Sensitivity $\left(\mathrm{pD}_{2}=-\log \mid \mathrm{EC}_{\mathrm{B}}\right)$ and the maximal response with the agonist $\left(\mathrm{E}_{\mathrm{man}}\right)$ were determined by sigmoidal curve fiting of individual curves (Graphpad Prism 2.01 software).

Fow-induced dilatation (IDD) was sudied by applying an intraluminal flow of 10 $\mu 1 /$ min represcnting a calculated wall shear stress value of 5 dyne/ $\mathrm{cm}^{2}$ at an intrahminall pressure of $80 \mathrm{~mm}$ Hg. Ateries were constricted either spontaneously wia myogenic tone or in combination with the tromboxane analogue 446619 to a level of approximately 50 60\% of the passive drancter. Since the dimeter obtained before application of sheat stress was the same for all vessels, the level of shear stress was also comparable for vessels of young and miclle age mice. The internal diameter at which maximal FTD occurred was recotded and percentage dilatation was calculated as a percentage of the passive internal diameter. At the end of the experiment, intraluminal flow was stopped and a constricuion retumed. Agonist-induced endothelium-dependent asodilator responses were evaluated by constructing at cumulative $\mathrm{CRC}$ for acetylcholine $(\mathrm{ACh}, 0.01-10 \mu \mathrm{MD})$. Time control experimetnts were conducted in order to check the stability of the FID responses. Three consccutive TID experiments yielded the same diameter responses to flow under control 
conditions. Therefore, experiments were repeated on the same single vessel in the presence of the NO synthase inhibitor $\mathrm{N}^{(1)}$-nitro-L-arginine (NLA, $30 \mu \mathrm{mol} / \mathrm{L}$ ). Similarly, endothelium-independent vasodilator responses were analysed by constructing a cumulative $\mathrm{CRC}$ for sodium nitroprusside (SNP, $0.1 \mathrm{nM}-100 \mu \mathrm{M})$ in the presence of both $30 \mu \mathrm{M} \mathrm{NLA}$ and $10 \mu \mathrm{M}$ indomethacin to block endogenous production of NO and prostaglandins.

At the end of the experiment, arteries were incubated in a $\mathrm{Ca}^{2+}$-free physiological salt solution containing sodium nitroprusside $(10 \mu \mathrm{mol} / \mathrm{L})$ at an intrahuminal pressure of $80 \mathrm{mmHg}$ to obtain the passive diameter. A passive pressure-diameter telationship was established by recording the diameter during stepwise $(10 \mathrm{mmHg})$ increases in intraluminal pressures from 20 to $100 \mathrm{~mm} H \mathrm{Hg}$. After each step the vessel was allowed to equilibrate until a stable diameter was reached. Wall thickness was recorded to calculate incremental elastic modulus and circumferential wall sttess. After the experiment, the arteries were fixed during 30 minutes at a transmural pressure of $80 \mathrm{mmHg}$ in $4 \%$ phosphate-buffered formaldehyde, and stored in $70 \%$ ethanol solution.

\section{Calculation of Parameters and Morphometry}

Circumferential wall stress (CWS) and incremental elastic modulus $\left(E_{\text {sut }}\right)$ were calculated as described. ${ }^{223}$ Morphometry of fixed vessels was done as described. ${ }^{22.3}$

Drugs

U46619 was purchased from Calbiochem, Damstad, Gemany. Acetylcholine was purchased from Serva Feinbiochemica, Heidelberg, Germany, L-phenylephrine, norepinephrine, $\mathrm{N}^{\mathrm{u}}$-nitro-I-arginine, sodium nitroprusside and indomethacin were purchased from Sigma Chemical, St Louis, USA.

\section{Siltistios}

Results are expressed as means \pm SEM. In all experiments, n equals the number of mice and vessels. Statistical significance of differences between sets of data was determined by ANOVA with post-hoc Student-Newman-Keuls test. A $P$-value of $<0.05$ was considered to indicate a significant difference.

\section{Results}

\section{General Observations}

Young mice had a significantly lower body weight than middle age mice (Table 8.1). Littet size and the number of viable fetuses in the uterus were significantly higher in young late pregnant mice compared to middle age late pregnant mice (Table 8.1). Mean cardiac and placental weights were comparable for both age groups (Table 8.1), but maternal body weight gain during pregnancy was larger for young than middle age mice.

\section{Ulerin Arsery Structure of Nonpregnam Mice}

Uterine arteries were significantly larger in middle age than young nonpregnant mice as shown in the pressure-diameter curves (Figure 8.1A). Passive internal diameter (at 80 
$m m H g$ was $233 \pm 9 \mu m$ for young mice and $286 \pm 11 \mu \mathrm{m}$ for middle age mice. Arterial wall elasticity was similar for the two mouse groups as reflected by compatable relationships berween incremental elastic modulus and circumferential wall stress (Figure 8.1B). Mediat cross-sectional area was significandy larger for utetine artenies of middle age mice (3.6t0.4 $\left.x 10^{3} \mu \mathrm{m}^{2}\right)$ compared to young adult mice $\left(2.4 \pm 0.3 \times 10^{3} \mu \mathrm{m}^{2}\right.$, Figure $8.1 \mathrm{~A}$, insert).

Table 8.1. Genchat characteristics and reproducrive performance of young and middle age mice.

\begin{tabular}{|c|c|c|c|c|}
\hline & \multicolumn{2}{|c|}{ Young } & \multicolumn{2}{|c|}{ Middlle Age } \\
\hline & nonpregnant & late pregnant & monpregnant & late pregnant \\
\hline Age (wk) & $1.8 \pm 1$ & $16 \pm 1$ & $63 \pm 3$ & $53 \pm 1$ \\
\hline Bodyweight $(g)$ & $23 \pm 1$ & $38 \pm 2 *$ & $28 \pm 1$ & $35 \pm 1 *$ \\
\hline Litter stze $(n)$ & NA & $8 \pm 1$ & NA & $4 \pm 1+$ \\
\hline Visble feruses (n) & NA & $8 \pm 1$ & $\mathrm{NA}$ & $3 \pm 1 \div$ \\
\hline Heatr weight (mg) & $125 \pm 5$ & $140 \pm 7$ * & $123 \pm 10$ & $163 \pm 11 *$ \\
\hline Placencal weight (mo) & $N A$ & $109+3$ & NA & $124 \pm 4$ \\
\hline Fetal weiglut (mg) & NA & $846 \pm 84$ & $\mathrm{NA}$ & $1013 \pm 343$ \\
\hline
\end{tabular}

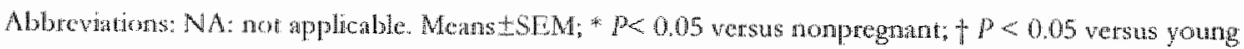

A

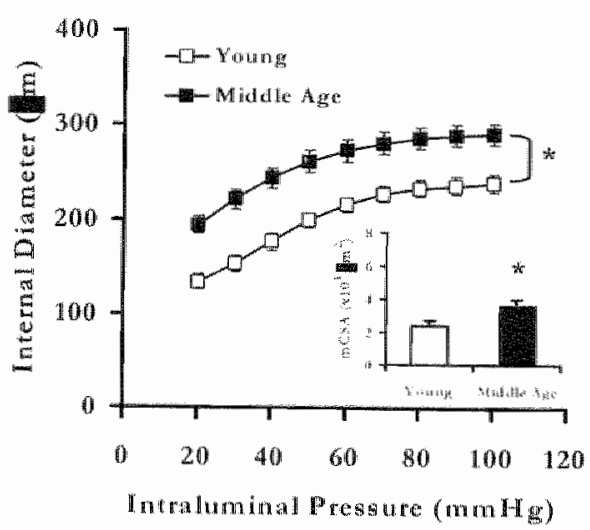

B

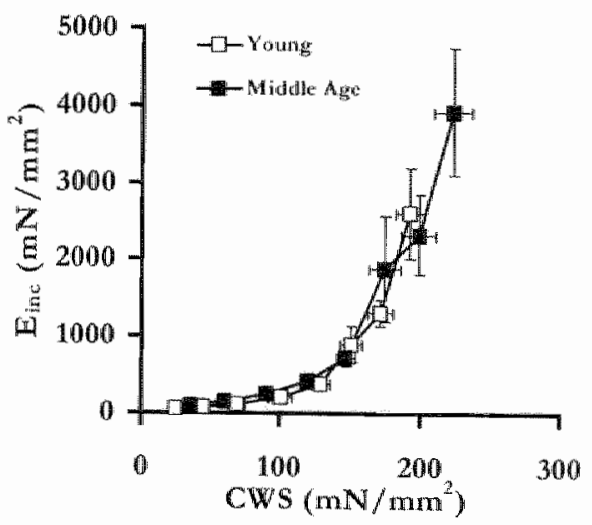

Figure 8.1. Uterine antery structure and etasticity of non pregant mice. (A) Dessure-diameter relationshipe in

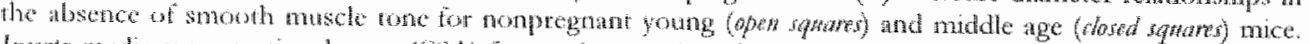

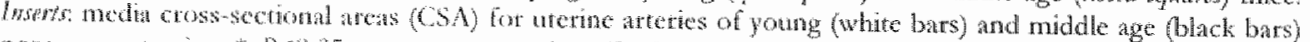

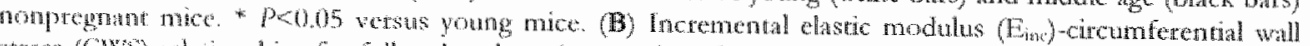

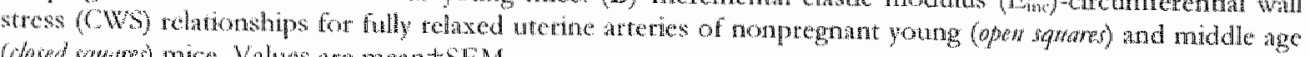

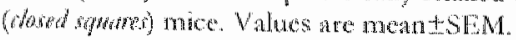

\section{Vasoconstrictor Responses of Nontpregnant Mive.}

Srep increases in intraluminal pressure caused a constriction from approximately 60 mm $\mathrm{mg}$ in uterine artcries of nonpregnant mice of young and middle age mice (Figure 8.2, wpper paned. All arteries of young mice developed a myogenic constriction, whereas only 4 out 8 arteties of middle age mice responded to intraluminal pressure. Consequently, 
percentage myogenic tone was significanty reduced in middle age nonpregnant mice compared to young nonpregnant mice $(19 \pm 7 \%$ versus $40 \pm 5 \%$, respectively). The maximal vasoconstriction induced by $\mathrm{NE}$ was comparable in young and middle age mice $(54 \pm 6 \%$ versus $57 \pm 4 \%$, respectively, Table 8.2). The sensinivity to the carecholamine did not differ between the two groups (Table 8.2).

\section{Waroditator Responses of Nompregnant Mice.}

The maximal vasodilatation induced by $\mathrm{ACh}$ was comparable in young and middle age mice (96.2\% versus $95 \pm 2 \%$, respectivelly, Figure 8.3 and Table 8.2 ). The sensitivicy to the endothelium-dependent agonist was similar in uterine arteries of bodh groups (Table 8.2). In young nonpregnant mice, nitro-L-arginine (NLA; $30 \mu \mathrm{M}$ ) significantly reduced the maximal dilator response, but not the sensitivity to ACh (Figute 8.3A; Table 8.2); while in middle age nonpregnant mice NLA markedly reduced the sensitivity to ACh (Figure 8.3B; Table 8.2).

All vessels dilated in response to SNP $(0.1 \mathrm{nM}$ to $100 \mu \mathrm{M})$ during preconstriction. Maximal endothelium-independent dilatations and sensirivity to the NO donor were comparable in uterine arteries of young and middle age mice (Table 8.2).

Application of a shear stress of 5 dyne $/ \mathrm{cm}^{2}$ resulted in a dilatation, that was comparable for young and middle age nonpregnant mice (Figute 8.4). Inhibicion of nitric oxide synthase with NLA (30 $\mu \mathrm{M})$ significandy reduced fow induced dilatation in uterine arteries of young nonpregnant mice, but not in middle age mice (Figure 8.4 ).

\section{Pronang-Related Cbanges in Young Mine.}

In young mice uterine artery internal diameter increased $60 \%$ from $233 \pm 9$ to $375 \pm 16 \mu \mathrm{m}$ at 0.9 gestation (Figure $8.5 \mathrm{~A}$ ). Arterial elasticity was unaltered during pregnancy (data not shown). Significant medial hypertrophy occurred in the utetine artery at 0.9 gestation; media cross-sectional atea incteased $150 \%$ from $2.4 \pm 0.3$ to $6.0 \pm 0.7 \times 10 \% \mathrm{~m}^{2}$ (Figute $8.5 \mathrm{~A}$, insert). Myogenic tone, constrictor tesponses to $\mathrm{NE}$, and dilator responses to $\mathrm{ACh}$ and $S \mathrm{NP}$, expressed as percent changes, were all similat in uterine arteries of late pregnant young mice compated to nonpregnant young mice (Figure 8.2 , lower panel Table 8.2 ).

\section{Pregnang-Related Cbanges in Middle age Mice.}

Uterine artery outward remodeling was significantly reduced in middle age mice compared to the young animals. Passive intemal passive diameter increased from $286 \pm 11$ to $342 \pm 15$ um at late pregnancy, representing only a $20 \%$ increase. (Figure 8.5B). Besides a matked reduction in outward remodeling, medial hypertrophy was substantally reduced in uterine arteries of midlle age mice compared to their younger counterparts. Medial crossm sectional area increased only $30 \%$ at late pregnancy (from $3.6 \pm 0.4$ to $4.7 \pm 0.5 \times 10^{3} \mu^{2}$, Figure $8.5 \mathrm{~B}$, insert).

Myogenic tone of the uterine artery significandy increased ac 0.9 gestation in miclde age mice (Figure 8.2, fower panel, Table 8.2). Constrictor responses to NE, and dilator responses to $\mathrm{ACh}$ and $S \mathrm{NP}$ were similat in uterine arteries of middle age late prognant mice compared to nompregnant mice (Table 8.2). 
A

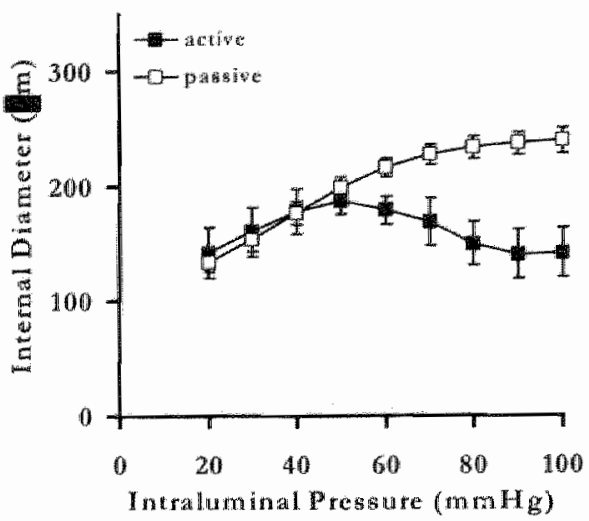

C Young Late Pregnant Mice

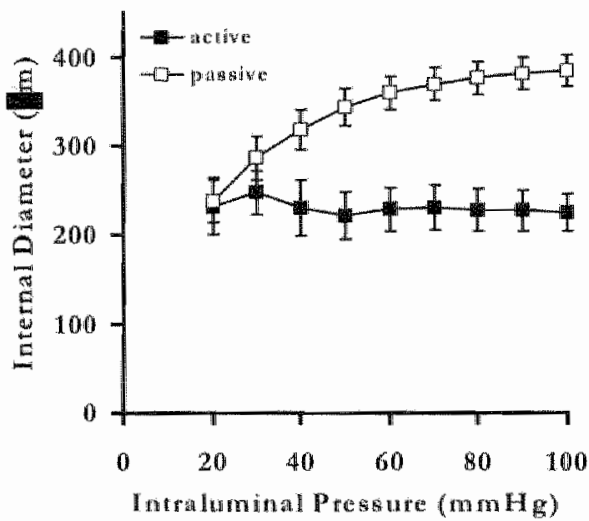

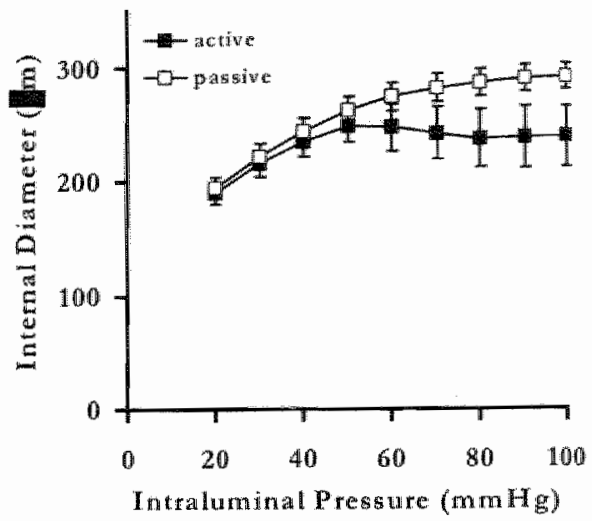

$\mathrm{D}$

Middle Age Late Pregnant Mice

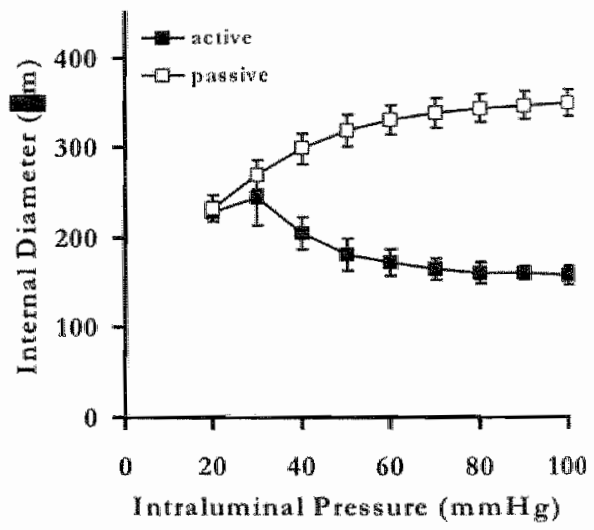

Figure 8.2. Pressute-1ndued myogenic constiction in uterine arterics of nonpregnant mice. (A) Pressure-

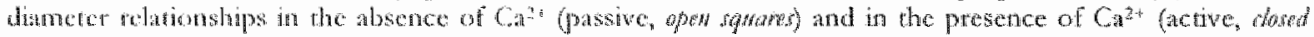

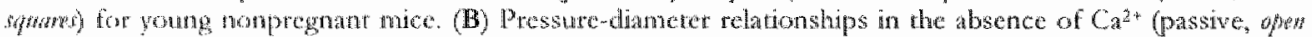

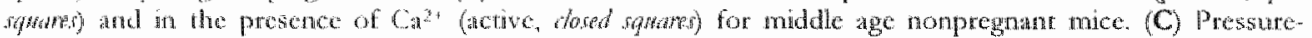

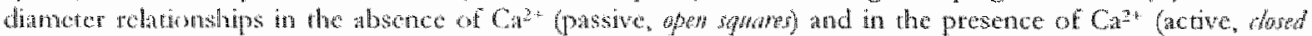
motorng) for young lare pregnant mice. (D) Pressure-diameter relationships in the absence of $\mathrm{Ca}^{2+}$ (passive, open

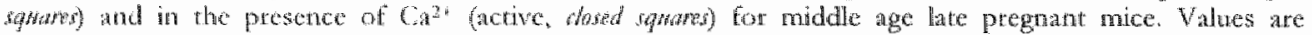
meandslikl. 
A

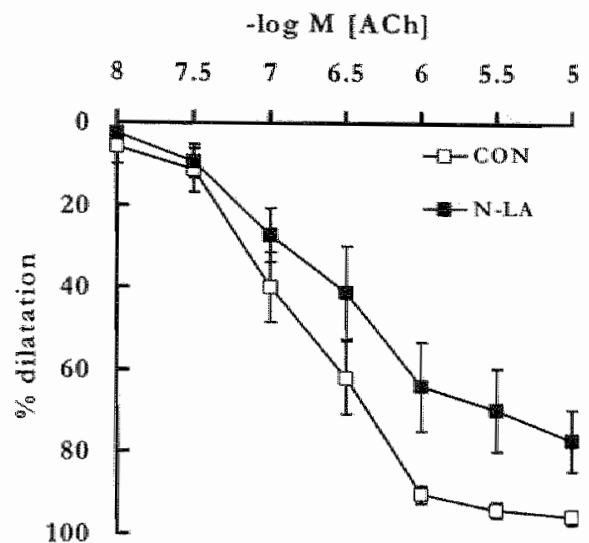

B

Middle Age Mice

$-\log \mathrm{M}[\mathrm{ACh}]$

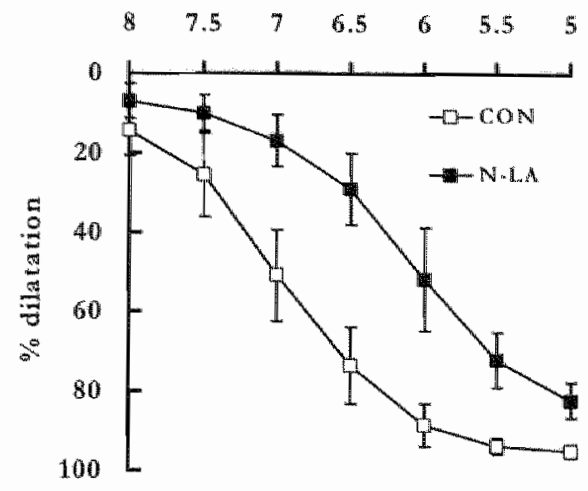

Figure 8.3. Concentration-tesponse curves (CRC) for acetylcholine in uterine atteries of nonpregnant maice under control conditions (open squares) and in the presence of $30 \mu \mathrm{M}$ NLA (dosed sowamen) for young nonpregnan mice (A). CRC under control conditions (open squares) and in the presence of $30 \mu \mathrm{M} N \mathrm{NLA}$ (dosed squares) fort middle age nonpregnant mice (B). Valucs ate mean \pm SEM.

Table 8.2. Constrictor and dilator properties of uterine atteries from nonpregnant and late prognant goung and middle age mice.

\begin{tabular}{|c|c|c|c|c|}
\hline & \multicolumn{2}{|c|}{ Young Mice } & \multicolumn{2}{|c|}{ Middle Age Mice } \\
\hline & notrpregnant & late pregnant & nonpregnant & late pregnant \\
\hline d/ $M T$ & $40 \pm 5$ & $42 \pm 5$ & $10 \pm 7 t$ & $54+3$ \\
\hline $\mathrm{pD}_{2} \mathbb{N E}_{4}$ & $6.73 \pm 0.06$ & $6.69 \pm 0.14$ & $6.42 \pm 0.22$ & $6.59 \pm 0.20$ \\
\hline$E_{\mathrm{Nax}}(0) \mathrm{NE}$ & $54+6$ & $58 \pm 4$ & $57 \pm 4$ & $53 \pm 3$ \\
\hline $\mathrm{pD}_{2} \mathrm{ACh}$ & $6.84 \pm 0.17$ & $6.29 \pm 0.12$ & $7.04 \pm 0.20$ & $7.22 \pm 0.32+$ \\
\hline$E_{\text {sin: }}(0) \mathrm{ACh}$ & $96 \pm 2$ & $95 \pm 1$ & $95 \pm 2$ & 99 止1 \\
\hline $\mathrm{PD}=\mathrm{ACH}$ as NLA & $6.48 \pm 0.21$ & $6.33 \pm 0.11$ & $0.11 \pm 0.18 *$ & $6.68 \pm 0.39$ \\
\hline$E_{\max }(\%) A C h+N L A$ & $77 \pm 7 *$ & $62 \pm 13$ & $82 \pm 4$ & $90 \pm 5$ \\
\hline $\mathrm{pD}_{2} \mathrm{SNP}$ & 7.3310 .12 & $6.91 \pm 0.23$ & 7.0040 .13 & 7.0210 .34 \\
\hline $\mathrm{E}_{\max }(\% \mathrm{SNP}$ & $68 \pm 0$ & $74 \pm 8$ & $54 \pm 7$ & $72+16$ \\
\hline
\end{tabular}

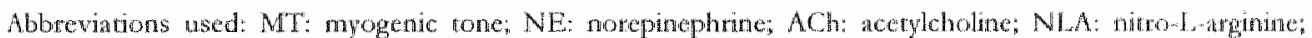

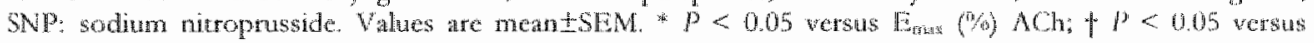
nonpregnant young and late pregnant middle age mice; $\neq P<0.05$ versus late pregnant young mice. 


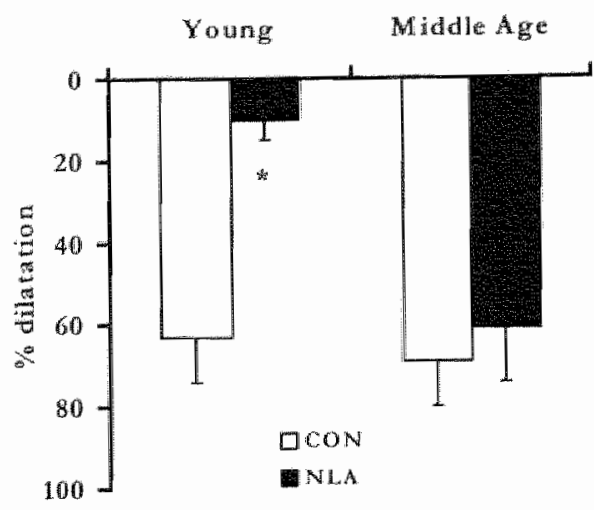

Figure 8.4. Mow-induced dilatation for uterine arteries under control conditions (opes bays) and in the presence of $30 \mu M$ NLA (wosed bart for young and middle age nonpregnant mice. An iniual shear siress of $5 \mathrm{dyne} / \mathrm{cm}^{2}$ was applied to whe ressels by a constant intraluminal pressure. * p<0.05 wersus NLA. Values ate meantSEM.

A Young Mice

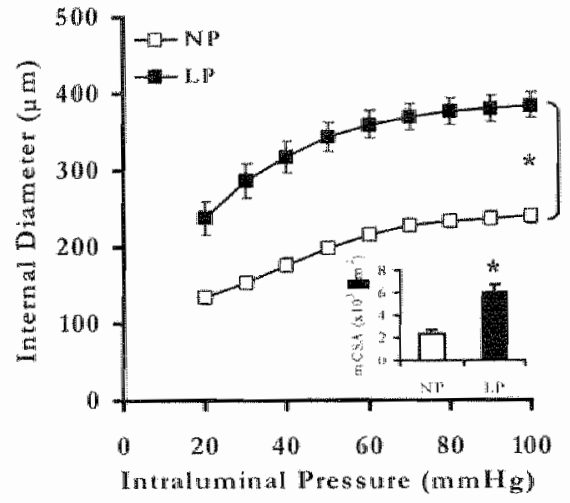

$\mathbf{B}$ Middle Age Mice

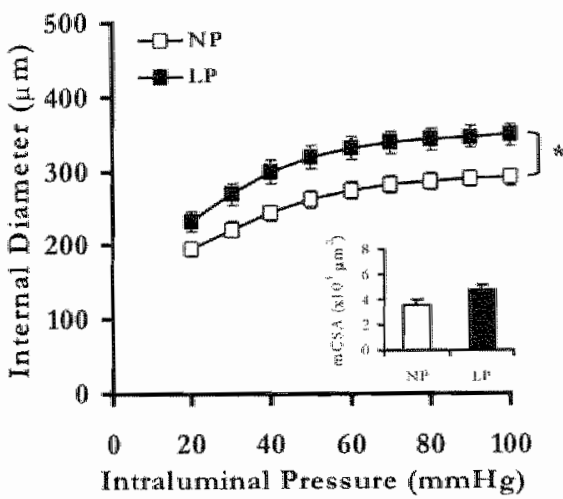

Figure 8.5. Pregnancy-related uterine attery stnictural changes. Pressure-diameter rellationships in the absence

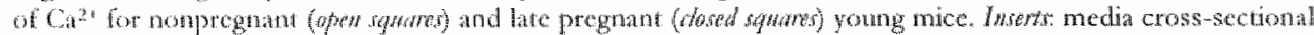
aress (CSA) for uterine arceries of nompregnant (white bars) and late ptegriat (black bars) young mice *

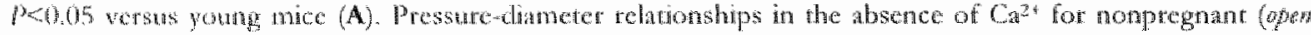

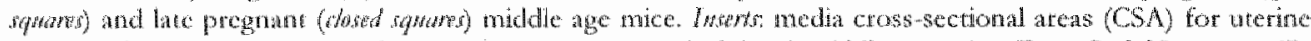
arteries of nompregnatur (white bars) and late pregnant (black bars) middle age mice (B). ${ }^{*}$ P<0.05 versus NP. Values inc meantsits.

\section{Discussion}

In utcrine arteres of 58 weeks old mice (1) shear stress-induced NO release and (2) pregnancymrelated remodeling were reduced. Altered flow-induced vasodilatation and blunted uterinc arteriogenesis were associated with impaired pregnancy outcome.

Using an in sitw "perfusion fixation method", Van der Heijen and colleagues demonstrated blunted utetine artery remodeling in 40 -weeks old late pregnant mice 
compared to 12-weeks old young adult late pregnant mice. ${ }^{56}$ They did not determine whether this diminished uterine artery remodeling capacity was due to an age-dependem change in endothelial function or smooth muscle cell activity. In this study we used the pressurized artery method (arteriograph setup) to measure acute arterial function and morpho-mechanical arterial properties in isolated vessels. We measured in young (1) weeks) and middle age (58 weeks) mice: 1) acute urerine artery tesponses to pressure (myogenic constriction), flow or shear stress (flow-induced dilatation) and agonists (norepinephrine, acerylcholine and sodium mitroptusside), 2) uterine artery remodeling (diameter and medial mass changes) at 0.9 gestation, and 3) pregnancy ourcome (number of riable fetuses).

With age, the vascular wall undetgoes struchiral changes such as increased stiffness, medial thickening and luminal diameter enlargement. ${ }^{369}$ In line with these abservations, uterine arteries of middle age nonpregnant mice had wider diameters and greater medial cross-sectional areas compared to uterine arteries of young nonpregnant mice. Uterine arterial elasticity was however comparable between young and middle age mice. Agingrelated arterial wall stiffening may require a longer period of time. Vascular aging is associated with functional changes at the level of all three layers of the arterial wall of both large conduit arteries and small resistance arteries. ${ }^{355}$ Age-related endothelial dysfunction has been characterized by diminished agonist-induced vasodilatation ${ }^{527}$ and flow-induced dilatation. ${ }^{37}$ The reduced vasodilatation is possibly due to an altered balance between endothelium-derived relaxing factors (og. NO) and constricting factors (e.g. endoperoxides). Furthermote, elevated oxidative stress plays a critical role in the deleterious effects of aging on the endothelium via enhanced scavenging of $\mathrm{NO}$. Changes in vascular reactivity to constrictor agents may be due to a combination of smooth muscle cell alteration and a reduced vasodilator function of the endothelium. ${ }^{32}$ The $\mathrm{C57BL} / 6 \mathrm{~J}$ mice, which we studied, have a reported life span of $>120$ weeks. The mice we studied thus represent young adult and middle age conditions and not geriatric conditions. We found comparable flow (shear stress)-induced uterine artery dilatations in young and miclde age mice. However, shear stress-induced NO release was significantly teduced in middle age mice as evidenced by the lack of an inhibitory action of $\mathrm{N}^{\text {ti }}$-nitro-L-arginine in uterine arteries of middle age mice. Manntenance of flow-induced dilatation, despite blockade of nitric oxide synthase, suggests compensation by other endothelium-derived relaxing factors. Likely candidates are bradykinin, ${ }^{2.3}$ prostaglandins, ${ }^{524}$ and endotheliumderiwed hyperpolarizing factor (EDHF), 2*4,246

Acetylcholine-induced endothelium-dependent vasodilatations were comparable and were sensitive to $\mathrm{N}^{\omega}$-nitro-L-arginine in uterine arteries of both age groups. Also, responses to Na-nitroprusside wete comparable in young and middle age micc. Collectively these findings indicate that the signaling cascade leading to the phosphorylation of endothelial nirric oxide synthase (eNOS), which mediates endothelial responses to shear stress, ${ }^{16 i i}$ is selectively impaited in uterine artertes of middle age mice. This is in line with the reduced shear stress-induced eNOS phosphorylation that was recently observed in mesenteric resistance arteries of aged rats. ${ }^{55}$. $\mathrm{MlsO}$, Hoffman and colleagues reported failute of shear stress to stimulate, phosphorylate and upregulate eNOS in human umbilical vein endothelial cells aged in cell culture. ${ }^{22_{3}}$ 
Elewated blood flow, and hence increased wall shear stress, is a major determinant of arterial remodeling. ${ }^{311,434}$ This flow-related arterial remodeling has been demonstrated to be endothelium-dependent. ${ }^{312}$ During pregnancy, blood flow through the utenine circulation increases substantially. ${ }^{456}$ Due to this increase in wall shear stress the uterine artery undergoes a structural increase in diameter, termed outward remodeling, and smooth muscle cell or medial hypertrophy. $223,408,561$ In the present study, we observed in middie age mice a diminished uterine artery remodeling (reduced luminal expansion) at 0.9 gestation. Our observation of blunted flow-induced arterial remodeling with aging is consistent with previous studies in carotid and mesenteric arteries of the rat. 301,030 The trigget for ontward arterial temodeling is most likely increased wall shear stress. Tuttle and colleagues have shown that the initial shear stress stimulus is proportional to the inctease in lumen diameter during collateral development in mesenteric arteries in young rats. ${ }^{55.3}$ Langille and colleagues previously suggested that with increasing age higher shear stress levels are required to trigger remodeling. ${ }^{3 / 1}$

Controversy exists about the tole of nitric oxide and eNOS in vascular remodeling. eNOS is known to be regulated by shear stress in the endothelium ${ }^{109}$ and its expression varies with the shear stress level ${ }^{555}$ An important role for eNOS and NO in flow-related arterial remodeling in large arteries has been suggested by several studies. ${ }^{361,546,547}$ However, in ligated mesenteric resistance arteries, flow-induced remodeling was not suppressed during chronic L-NAME treatment in rats. ${ }^{84}$ This paradox might find its origin in endothellium-derivecl mediators that are resistant to blockade and deficiency of eNOS and which are more prominent in small muscular arteries than in latge elastic conduit vessels. In uterine arteries of middle age mice, mechanisms that are resistant to $\mathrm{N}^{(1)}$-nitro-L-argininc could sustain flow-induced vasodilatation but not pregnancy-related remodeling.

The number of viable fetuses was significantly reduced in middle age term pregnant mice compared to young animals. Van der Heijden and colleagues have shown that at day 11. of murine pregnancy, litter size and the number of viable fetuses was similar in both mature and young mice. Thus, the urerine blood flow rise at this time point may be sufficient to allow normal placentation and eatly embryo development, however when blood flow demands are high at late pregnancy, the number of viable pups decreases progressively suggesting a causal relationship between blunted flow-related uterine artery temodeling and pregnancy outcome. ${ }^{561}$ Retrospective population-based studies indicate at higher prevalence of perinatal complications in pregnant women ower 35 years of age, $16,3,37,399$ which may be related to the increased incidences of chronic hypertension and diaberes mellinus at these ages. 17.564

In conclusion, aging blunts pregnancy-related uterine artery remodeling, which appears to contribute to a poorer pregnancy outcome. A selective reduction of shear stress-induced nitric oxide release might be the cause for this diminished outward remodeling. 
Chapter 9

\section{Acute and Chronic Adaptive Responses to Altered Blood Flow in Large and Small Arteries}

Rob H.P. Hilgers, Paul M.H. Schiffers, Jos P. Wesselinan, Jo G.R. De Mey.

Department of Phamacology \& Toxicology, Cardiovascular Research Instirute Mastricht (CARUM), Universiteit Mastricht, the Netherlands. 


\section{Abstract}

In analogy to arterial hemodynamic responses to chronic pressure alterations, blood vessels have adaptive mechanisms to cope with sustained changes in arterial blood flow. This so called flow-induced atterial temodeling can occus in warious physiological events, such as during pregnancy and long-term exercise, and in pathological situations, such as peripheral and coronary occlusive disease and atherosclerosis. Flow-induced remodeling of the arterial wall results in diameter adjustments that correct wall shear stress (WSS) roward nomal values. Accordingly, chronic blood flow elevations lead to a widening of the arterial structural lumen diamerer, whereas chronic blood flow reductions lead to narrowing of yessel diameter. Arterial responses to flow changes involve two processes: acute vasomotor tesponses and chronic wall reshaping or remodeling. Both processes ate regulated by WSS that is sensed by the endothelium. The majority of studies on flowrelated anterial remodeling have focussed on the role of endothelium-derived factors. However, a growing body of evidence indicates that blunted acute vasomotor responses to WSS do not necessarily entail a defect in chronic wall restructuring. Either other mediators ate involved, or WSS is transmitted from the cell surface and cytoskeletal filaments to the nucleus, independent from mechano-chemical signaling processes, suggesting that acute and chronic responses to WSS can operate independently from each other.

In this review article we define the processes of flow-related arterial remodeling and focus on animal models representing expetimental and physiological settings of chronic blood flow alterations in large and small arteries. We describe the spatial events in flowrelated arterial remodeling and acldress the role of acute vasomotor responses, endothelium-derived factors, and the local renin-angiotensin system in chronic structural remodeling of the arterial wall in response to long-term blood flow changes. 


\section{Introduction}

It is well established that arteries sense and respond to modification of hemodynamic conditions, such as changes in blood pressure and flow. These mechanical stimuli are sensed as circumferential wall stress (CWS, pressure) and wall shear stress (WSS, flow). CWS is the tangential stress induced by the transmural pressure. It is derived from the law of Laplace:

$$
C W S=\frac{T P \cdot r}{W t}
$$

where TP is transmural pressure, $\mathrm{r}$ is radius and Wt is wall thickness. On the other hand, wall shear stress is the frictional force of the dragging blood flow. It is defined by the law of Pousseuille:

$$
W S S=\frac{4 \cdot \eta \cdot Q}{\pi \cdot r^{3}}
$$

in which $\eta$ is the blood viscocity, $Q$ is the blood flow velocity and $r$ is the radius of the attery. The responses to these stresses consist of acute and chronic components. Acutely, the vasomotor tone changes (e.g. pressure-induced (myogenic) tone and flow-induced vasodilatation), while chronic exposute to mechanical stresses leads to a structural adaptation of the arterial wall, which ultimately restore mechanical stresses towards normal values.

Several temodeling responses can be distinguished (Figure 9.1). The classification proposed in 1996 by Mulvany, and other leaders in the field of hypertension-induced resistance-sized artery remodeling, ${ }^{379}$ is based on the changes of the structural arterial lumen diameter (inward or outward) and on changes in wall mass (increased: hypertrophic, decreased: hypothrophic, and unchanged: eutrophic). For example, an increase in lumen diameter without a change in medial cross-sectional area, would be denoted as an ourward eutrophic remodeling. Note that an eutrophic remodeling can result from either an inward remodeling with an increase in wall thickness or an outward remodeling with a decrease in wall thickness, indicating that a change in wall mass may aiso involve rearrangement of existing wall material or a balance between cell growth and cell death.

Our reasons to write this review on flow-induced arterial remodeling are two-fold. Firstly, a growing consensus is emerging about arterial remodeling during hypertension. In analogy to this pressure-induced atterial remodeling we want to propose a gencral concept of flow-related arterial remodeling of small and large arteries. Secondly, the increasing number of studies using genetically modified animals yielded a large body of information 
on the role of specific proteins and mediators in acute and chronic adaptive responses of arteries to altered blood flow. From these studies a growing body of evidence is emerging that suggest that optimal acute flow-induced vasodilatation is not required for chronic structural arterial remodeling in response to altered blood flow. This observation inspired us to address the role of endothelium-derived vasoactive factors in arterial diameter adjustments to chronic blood flow changes in animal models.

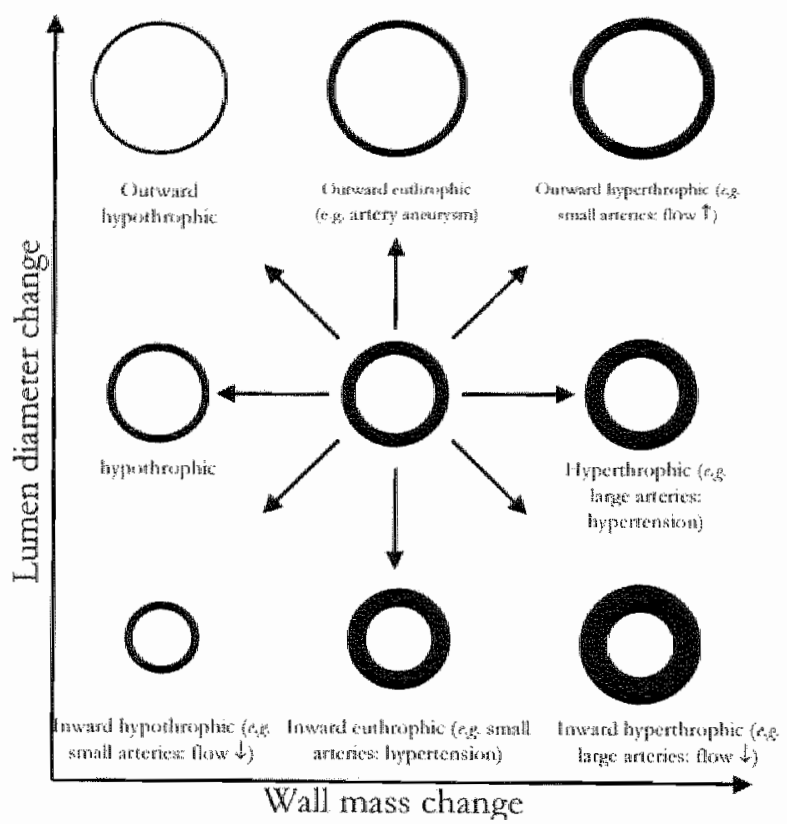

Figure 9.1. Arterial remodeling evenins (adapted from ${ }^{37}$ ) resulting from exposure to chtonic alletarions in ransmual pressure and/or blowd flow. On the wertical axis are lumen diameter changes denoted as either inward (decrease in diametcrit, outwand (increase in dismeter) or unchanged. Similarly, on the horizontal axis are wall mass changes termed hyporrophic, eutrophic, and hypertrophic as, respectively, a decreased, unchanged, and increased wall mass.

\section{Lessoms Learnt From Hyperlension Studies}

In hypertension, where arteries are chronically exposed to elevated transmural pressure, different kinds of adaptive atterial remodeling have been demonstrated in large and small arteries (Tigure 9.2). 1,46.446.453 Large arteries have a high content of extracellulat matrix components such as collagen and elastin. Their capacity to constrict to (neuro) humotal and mechanical factors is less than $10 \%$. Increased transmural pressure leads to an acute passive distension, which induces an elevation of circumferential wall stress. This signal is sensed, transduced, and eventually causes proliferation of cells and rearrangement of preexisting wall material, and increased extracellular matrix deposition, thus leading to outward hypertrophy. ${ }^{318,378,439}$ The overall effect is an increase in wall thickness that normalizes CWS as depicted in the upper part of Figure 9.2.

In arterioles, increases in transmural pressure initially induce an active vasoconstriction, which opposes the passive distension. During this myogenic response, the thickness of the arteriolar wall increases and CWS is normalized. Thus the CWS sensor will no longer be activated and the proliferative signaling will not occur. ${ }^{9}$ Because of the rearangement of dynamic focal-adhesion sites, by which the arterial cells are connected to their matrix, the maintained constriction will ultimately become structural, 
constricted either spontaneously via myogenic tone or in combination with the tromboxane analogue U46619 to a level of approximately $55 \%$ of the diameter in the absence of smooth muscle tone (passive diameter). Some arteries developed a myogenic rone strong enough such that no U46619 had to be added in order to reach the desired constriction level. Time control experiments were conducted in order to check the stability of the FID responses. Three consecutive FID experiments yielded the same diameter responses to flow under control condicions. Therefore, experiments were performed on the same single vessel under control conditions, in the presence of intraand extraluminally added $\mathrm{N}^{\mathrm{t}}$-nitto-L-atginine methyl ester (L-NAME, $100 \mu \mathrm{mol} / \mathrm{L}$ ), and repeated a third time in the presence of both L-NAME $(100 \mu \mathrm{mol} / \mathrm{L})$ and indomethacin $(10 \mu \mathrm{mol} / \mathrm{L})$. Experiments in the presence of the $\mathbb{B}_{2}$ receptor antagonist HOE-140 $(0.1$ umol/L) were performed on a different set of $T K^{+/+}$mice. Concentrations of L-NAME, indomethacin and HOE-140 were selected on the basis of the literature.

At the end of the experiment, arteries were incubated with a $\mathrm{Ca}^{2+}$-free physiological salt solution containing sodium nitroprusside $(0.1 \mathrm{mmol} / \mathrm{I})$, and the pressure steps (10$140 \mathrm{mmHg}$ ) were repeated to obtain the pressure-diameter relationship in the absence of smooth muscle tone.

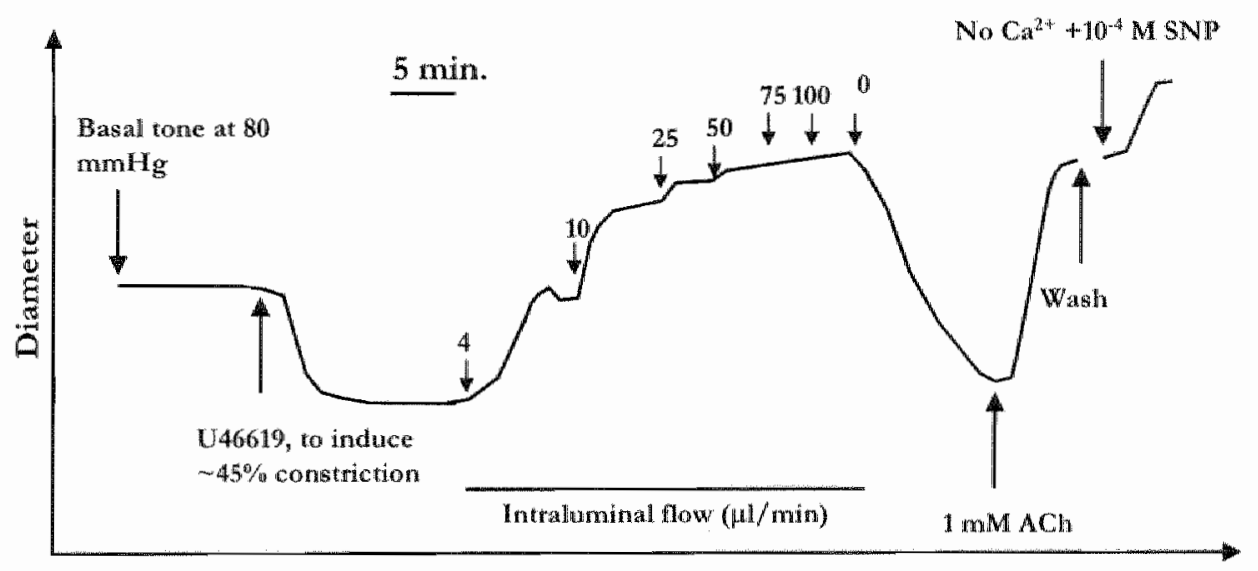

Tiine

Figure 4.1. Experimental schome to assess flow indaced wasodilaturon of isolated pre-constricted unctine arteries. Abbreviations used: ACh: acetylcholine; SNP; sodium nitrophusside.

Pressure and diameter changes were recorded using the Biopac data acquisition system (MP100; Biopac, La Jolla, CA), and analyzed using Acqknowledge(B) sofware (Biopac). To determine the maximal lumen diameter, pressure-diameter curves were analyzed by a third-order polynomal curve fitting $\left[y=A+B x+C x^{2}+D x^{3}\right]$ of individual curves using Graphpad Prism ${ }^{\text {TM }} 2.01$ software.

Myogenic tone was calculated using the formula: $\% \mathrm{MT}^{\circ}=$ (passive diameter measured diameter)/passive diameter) $\times 100$. Flow-induced dilatation was expressed as percentage dilatation of U46619-induced and myogenic tone. ${ }^{\text {ins }}$ 
leading to inward eutrophic remodeling (Figure 9.2 , lower part) ${ }^{17}$ Small arteries (0: 100 $300 \mu \mathrm{m})$ possess a weak myogenic reactivity and consequently display an intemediate type of remodeling.

In this concept, CWS is the sensed and controlled variable. In large arteries the hypetrophy normalizes the elevated CWS, and in arrerioles this is accomplished by means of the myogenic tone. The extent to which an artery can constrict in general, and in response to an increase in CWS in particular, then determines the type of the adaptive structural response. Whether vasoconstrictors can migger hypertrophy and how arteriolar constriction is ultimately fixed by a reduction of the structural lumen diameter, remain areas of active research.

In an effort to explain the structural adaptation of artenies to long term changes in blood flow, we used this pressure-induced remodeling theoty as a stating point.

Hypertrophic and Eutrophic Inward Remodeling in Hypertension.
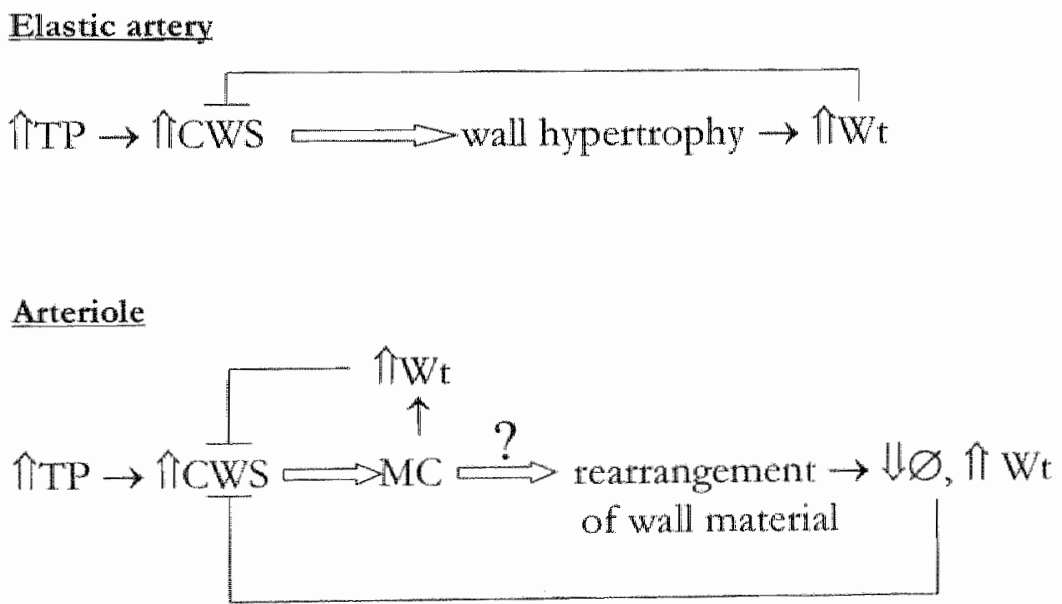

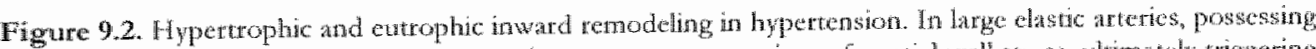

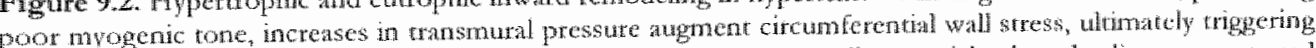
cell proliferatwe responses and reatrangement of pre-existing wall material, thus lending fo ourwarc

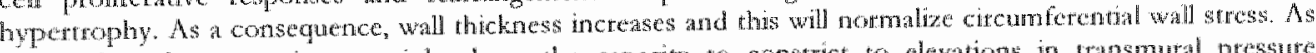
opposed to harge atateries, arterioles have the capacity to constrict to elevations in transmaral pressute

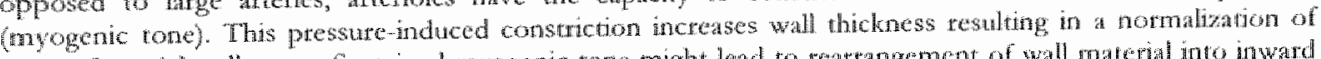

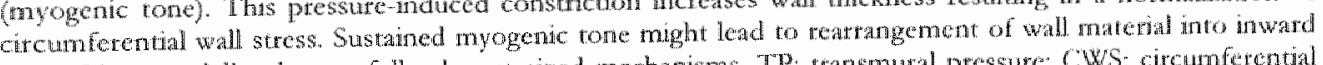
eutrophic remodeling by not fully characterized mechanisms. TP: transmat prosure; Cws: circumferental

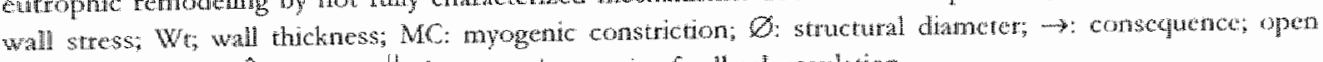
arrow: stimulation, Dincrease; U: decrease; 1 : negative foedback reguation. 


\section{Flon-Indwed Arterial Ranodeling a Defrnition}

Flow-induced arterial remodeling is the process involving artetial adaptations to chronic alterations in flow (shear stress) imposed on the endothelium, vascular smooth muscle cells (VSMCs), adventitia and extracelluar matrix composing the arterial wall. The wimate result is a nomalization of both CWS and WSS.

Remodeling of an arterial bed in response to changes in blood flow can occur via wo mechanisms: capillary network expansion via the sprouting of new capillaries from existing capillaties (angiogenesis) and enlargement of existing collateral arterioles (arteriogenesis), ${ }^{13}, 25 \%$ In this review we will focus on the processes of arteriogenesis or shear stress-induced arterial remodeling, and not on ischemia-induced angiogenesis.

Changes in archirecture of the arterial wall to alterations in hemodynamic stimuli occur under physiological as well as under pathological conditions. Situations in which flow-induced arterial remodeling occurs under physiological conditions are pre- and postnatal development, chronic exercise (leading to outgrowth of skeletal muscle circulation and outward remodeling of the muscular feed arteries), and pregnancy (sprouting of fetoplacental arteries and upstream uterine artery remodeling). Certain pathologies such as atherosclerosis, diabetes, and preeclampsia are associated with reduced or inadequate rissue perfusion leading to pathological adaptations of the vessel wall.

\section{Differewer betwen Swath and Lange Arteries}

Elastic and resistance-sized arteries differ in size, composition, function and local environment. ${ }^{45}$ Resistance-sized arteries have a higher density of VSMCs thata large elastic arteries, which have more extracellular matrix components such as collagen and elastin. VSMCs may be mote susceptible to arterial wall mass changes caused by altered wall stresses. Furthernore, many small atteries are densely innervated with sympathetic nerves, whereas conduit arteries are not. Acute flow-induced dilatation (FID), opposes myogenic tone, and is more nelevant and substantial in muscular, thin-walled arteries than in large arteries. For instance, FID in isolated pre-constricted mouse catotid arteries $( \pm 600 \mu \mathrm{m})$ is approximately 30 to $50 \mu \mathrm{m}$, representing 5 to $10 \%$ of the diametar.3.3. whereas pre-constricted isolated small mesenteric arteries ( $+150 \mu \mathrm{m}$ ) can dilate 50 to 60 $\mu \mathrm{m}$, representing up to $40 \%$ of the passive diameter. contribution of endothelium-derived vasonctive mediators regulating vasomotor tone depends largely on the vessel type. Nitric oxide (NO) is a potent vasodilator that is released in response to elevated WSS. In large artertes, such as the carotid artery, Dowinduced dilatation is manly depended on $\mathrm{NO}$ release, ${ }^{35 \%}$ and to a lesser extent on PGI and endothelium-derived hyperpolarizing factor (EDHF). However, in resistance arteries, the role of $\mathrm{NO}$ is less pronounced. In some arterioles, FID depends solely on the production of prostaglandins, ${ }^{291}$ or a combination of prostaglandins and EDHF 524 Furthermore, matrix metalloproteinases (MMPs) are proteases that can degrade exracellular matrix proteins. Since large arteries have a higher content of these cxtracellular matrix components, the effect of MMPs on vessel remodeling might be different in large arteries compared to small muscular arteries. Taken together, this difference in endothelial function between latge and small arteries might impose 
differental effects on the modes of flow-induced arterial remodeling, as far as arterial lumen ajustments and arterial wall mass changes ate concerned.

Before addressing the putative mediators involved in flow-induced temodeling, we describe some physiological and experimental setings of tow-induced arterial remodeling and focus on the sparial remodeling events of latge and small arteries in response to flow alterations in closer detail.

\section{Models of Flow-Induced Arterial Remodeling}

Chronic alterations in blood flow occur under physiological conditions such as during exercise and pregnancy (Table 9.1). During treadmill exercise in rats, a significant increase in blood flow (reaching up to 50-fold) to working skeletal muscle develops, 12 resulting in significant morphological changes in the vascular wall and in the structure of arteriolar networks.314,488 During pregnancy a drastic tise in uterine blood flow progressively develops. $160,355,456$ In order to accommodate the inctease in uterine blood flow, the uterine pasculature undergoes luminal. expansion, longitudinal growth and an increase in wall mass. ${ }^{107,223,224,395,344,406,408,561}$ Immediatedly after giving birth, uterine blood flow declines sharply, this thus represents a model of physiological blood flow reduction.

Table 9.1. Studies Investigating Models of Plyysiologital Flow-Relned Romodeling in Several Arteries and Specios:

\begin{tabular}{|c|c|c|c|}
\hline Artery Type & Species & Model & References \\
\hline \multirow[t]{3}{*}{ Aorra } & Guinea Pig & Pregnancy & 276 \\
\hline & Camb & Perinatal growth & 311 \\
\hline & Human. & Pregnancy & 133 \\
\hline \multirow[t]{4}{*}{ Carotid } & Rat & Pregnancy & 277 \\
\hline & Guinea Pig & Pregnancy & 277 \\
\hline & Ewe & Pregnancy & 195 \\
\hline & Hemary & Vexcerise & 484 \\
\hline \multirow[t]{2}{*}{ Pemoral } & Guincal Pig & Pregnancy & 275 \\
\hline & Human & Exercisc & 249 \\
\hline Skelctal muscle arterioles & Rat & Exereise & 488 \\
\hline \multirow[t]{7}{*}{ Uerine } & Mouse & Pregnancy & $223,224,501,571$ \\
\hline & Rat & Pregnancy & $107,304,406,408,577,518$ \\
\hline & Gainea Pig & Pregnancy & 145,363 \\
\hline & Rabbir & Pregnancy & 508 \\
\hline & Pig & Pregnancy & 199 \\
\hline & Ewe & Pregnancy & $11.168,195,279$ \\
\hline & Human & Pregnaney & 59 \\
\hline
\end{tabular}

Experimental models in which chronic altertions in arterial blood flow are induced by surgical manipulation, have been pplied to a broad range of arteries in several species (Table 9.2). Most expermental animal models to study arterial remodeling in response to chronic elevations in blood flow involve cteation of an arteriovenous fistula 
$(A V F)$, which can be performed in a number of large arteries such as the femoral artery $19,28,350,498,39,544,547$ and aortat $33,202,595$ in animals such as the baboon, rabbit, wat and mouse. A majot advantage of this method is that supraphysiological blood fow levels are obtained in large arteries. For example, an approximate 30 -fold inctease in iliac artery blood fow was obtained in monkeys ${ }^{6 \% 1}$ and an 11 -fold increase in carotid artery blood flow in the rat. $5 \%$ Another method is the undateral ligation of the carotid artery, first introduced by Guyton and Hartley, ${ }^{200}$ which leads to a reduction of blood flow in the ipsilateral (ligated) artery and a elevation in blood flow in the contralateral right carotid artery. Collaterals interconnecting the facial, cranial and cerebral areas, perfused by both catotid arteries form the anatomical basis for this shunting. $23 \mathrm{~A}$ comparable method was applied in mice by Kumar and Lindner, ${ }^{300}$ resulting in a complete cessation of blood tow in the ligated common carotid artery and a nearly doubling in blood flow in the contralateral carotid artery. Analogous to the unillateral carotid artery ligation, this surgical approach can be applied to side branches of the superior mesenteric artery, resulting in decreased blood flow in ligated mesenteric arteries and shunting of blood flow through collaterals resulting in flow-loaded mesenteric arteries.327,434,555.557 A number of nouse models exist in which flow-related arterial remodeling has been studied after targeted gene delecion, these are summarized in Table 9.3 and will be discussed.

Table 9.2. Srudes Investgating Expewimental Setrings of Flow Related Renodeling in Severat Arteries and speries

\begin{tabular}{|c|c|c|c|}
\hline Artery Type & Species & Model & References \\
\hline \multirow[t]{2}{*}{ Aorta } & Monse & AVI & 201,202 \\
\hline & Rat & $A V I$ & 33,595 \\
\hline \multirow[t]{7}{*}{ Canotid } & Dog & $A V T^{\circ}$ & 282 \\
\hline & Humar & Wigation & 257,296 \\
\hline & Mouse & Ligation & $\begin{array}{l}65,138,173,187,210,260,299,300,371,381,383 \\
463,479,502,503,522,59\end{array}$ \\
\hline & Rabbit & AVT & $119,348,350,546,547$ \\
\hline & Rithbit: & Ligation & $62,90,310-312,498$ \\
\hline & Rall & AVT & 530 \\
\hline & Rat & Cigations & $361,368,580$ \\
\hline Catchin & Rabibit & $A V I ;$ & 349 \\
\hline Coronatry & Dog & ligution & $73-75,591$ \\
\hline Cremaster masclo artetole & $\mathrm{Rat}$ & Orchidectomy & 578 \\
\hline \multirow[t]{3}{*}{ Fomotal } & Dog & Ligation & 97,458 \\
\hline & Rombir & Ligntion & $13,15,119,259$ \\
\hline & Ras! & Liginton & $121,329,558$ \\
\hline \multirow[t]{2}{*}{ Mesenteric } & Montaxe & Ligation & 326,327 \\
\hline & Rat & ligation & $71,84,434,435,552,527,586$ \\
\hline Penlmonary & Plg & $A H^{2}$ & 350 \\
\hline Radial & Human & AVI & 182,447 \\
\hline
\end{tabular}

Abbrewiations used: AVE; arteriowenous fistula. 


\section{Spatial Events in Flow-Induced Arterial Remodeling}

\section{Respowser to flow elevalions}

Acute WSS elevations initially cause a vasodilatation that tends to restore WSS in both large and small arterics (Figure 9.3). Flow-induced vasodilatation is mediated by endothelinm-derived mediators such as NO, PGI, EDHF, and bradykinin. Sustained elevated blood flow will eventually result in a structural luminal expansion, most likely resulting from rearrangement of existing wall mass leading to a thinner wall. This thinning of the arterial wall will increase the CWS, which triggers growth and proliferation of arterial wall cells. The resulting wall hypertrophy normalizes CWS. 1144 Hence, the overall outward hypertrophic response aims at nomalization of both WSS and CWS (Figure 9.4).

Table 9.3. Mouse Models in which Flow-Related Artertal Remodeling has been Studied After Targeted Gone Delletion

\begin{tabular}{|c|c|c|c|}
\hline Deleted Gene & Artery Type & Effect on Remodeling & Refertence \\
\hline Desmin & Mescrnteric & lexugerated monodeling & 327 \\
\hline Dystophin & Mesenteric & Reduced onward remodeling & 326 \\
\hline Vumentin & Carotid & Exaggerated outwatd remodeling & 479 \\
\hline Vitroncetin & Carovid & Increased neointima formation & 122 \\
\hline $\operatorname{MOS}$ & Carotid & Nonimsard remodeling & 463 \\
\hline MOS & Carotid & Increased neountima fomalion & 598 \\
\hline MOS & Carotad & Incteased inward remodeling & 508 \\
\hline MOS & Carotid & Increased neointima fotmation & 372 \\
\hline ETh receptor & Cattorid & Increased nepintima formarion & 381 \\
\hline MMP -9 & Catorid & Reduced noointima formation & 173 \\
\hline $\mathrm{FG} 12$ & Catotid & Normal responses & 522 \\
\hline P-selcotin & Cinotid & Reduced neointima formation & 290 \\
\hline P.All & Garatotid & Increased neointima formation & 122 \\
\hline Osteoponitin & Gatotid & Reduced in lammatory response & $383^{3}$ \\
\hline Tissude angotensin-converting enayme & Carotid & Decreased modial hyperirophy & 224 \\
\hline Tissue kallikrein & Uterine & Nomal Oumward nemodeling & 223 \\
\hline Issue angionensin-converang enzyme & Uterinte & Nomal Oumward semodeling & 224 \\
\hline
\end{tabular}

Outward hypertrophic remodeling is obsetved in both latge elastic arteries and in small muscular arteries. Zarins and colleagues showed that an AVF in primates led to hypertrophic ourward remodeling, cross-sectional area (CSA) increased due to VSMC hypertrophy, despite a decrease in arterial wall thickness. ${ }^{3}$ Similar findings wete obtained in carotid arterics with an AVH.350 Increases in wall shear stress tesult in vascular smooth muscle cell gene expression, such as the gene encoding for the nuclear transcription factor -fos, 202 initiating processes such as growth and matrix remodeling, which lead to flow-induced enlargement.

Also in small arteries outward hypertrophic remodeling is observed in response to chronic elevations in blood flow. $7,434,55,557$ The hypertrophy was due to SMC proliferation, because SMC number, as determined by a 3D-dissector technique, 
increased, and immunohistochemical assessment of proliferating cell nuclear antigen also showed an increase. ${ }^{71}$ The increase in wall mass was accompanied by a doubling of the DNA content of these flow-loaded vessels. ${ }^{434}$ VSMC reactivity to contractile compounds was increased compared to control arteries, due to the larger muscle mass. After 16 days, when outward temodeling was significant, desmin mRNA levels were reduced, indicative of VSMC de-differentiation. Endothelium-(in)dependent dilatations were not modified in these hyperperfused arteries. ${ }^{435}$ Furthermore, apoptotic cells were detected in the medial layer, ${ }^{71}$ suggesting both apoptotic and proliferative tesponses of the atterial wall. Also adventitial cell proliferation occured in the flow-loaded arteries, ${ }^{434,55}$ indicating that all three individual layers of the arterial wall seem to be directly or indirectly sensirive to the degree of WSS imposed.

Flow-Induced Vasodilatation.

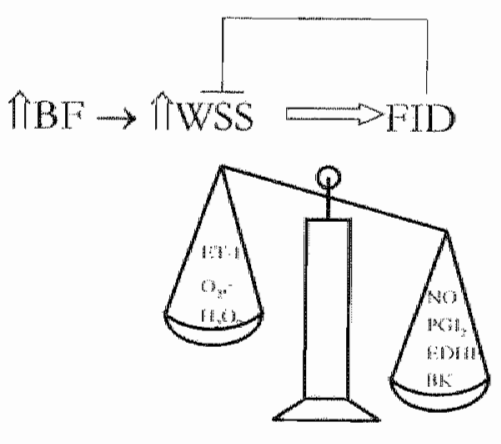

Figure 9.3. Fow-induced arterial wasodilataton in both small and latge arteries. Acute increases in bood flow clevate wall sheat stress which seimulates the release of endothelimaderwed wasodiator factors and might reduce the release of endothelumderived contraction factors.

Abbrevitions: BF" blood now FTD: Now-lnduced dilatation; WSS: wall sheat stress; $\rightarrow$ : conseguence; open arrow: stimulation; increase; 1 : nogatie feedback reguation; ET 1 ; andohelin-1; $\mathrm{O}_{2} \cdots$ superoxide anion; $\mathrm{H}_{2} \mathrm{O}_{2}$ : hydrogen peroxide : $\mathrm{NO}$ : nirtic oxide; PGIn" prostacydin; PDHF: endothelium derived hyperpolarimg fartor; $B$ : bradykinin.

A few complications arise when interpreting outward hypertrophic remodeling after drastic blood flow tncreases. Figure 9.4 suggests that sustained FID will lead to a reartangement of wall material. Fowever, a direct relation between acute FID and rearrangement has not been determined. Another discrepancy is the fact that large arteries have a limited FID that is insufficient to accommodate strong WSS elevations. During pregnancy uterine blood flow can increase 50-fold in tars, demanding a more than twofold increase in uterine artery lumen diameter to normalize WSS, which is never accomplished by FID. In several situations of endothelial dysfunction, eg. in diabetes and atherosclerosis, flow-induced arterial remodeling can still proceed. These observations suggest that arteries can follow a different strategy enabling a direct coupling between strongly elevated WSS and rearrangement of wall material leading to outward remodeling, that normalizes WSS. Analogous to Figure 9.4, this wall thinning will increase CWS and triggers a hypertrophic response of the arterial wall that normalizes CWS (Figure 9.5). 


\section{Responses to chronic flow reductions}

In general, chronic reductions in arterial blood flow lead to a reduction in lumen diameter that normalizes WSS (Figure 9.5). Vasomotor tone will shift towards more vasoconstriction, probably due to less FID. In parallel to sustained pressure-induced vasoconstriction, this narrowing of the lumen diameter will become structural, since the diameter can not be restored to control values after application of several wasodilatory compounds 3 days postligation in mesenteric arteries 434 and 7 days postligation in catotid arteries. ${ }^{62.462}$ This structural remodeling at day 7 coincided with an increase in cell death as shown in ligated tabbit arteries. ${ }^{30}$ In large arteries, such as the catotid artery, abrupt flow cessation leads to an initial enlargement in the first week post ligation followed by a progressive decrease in lumen area reaching a plateau after 4 weeks. ${ }^{187}$ The intima steadily increases throughout these 4 weeks. Formation of a neointima results in a reduction of the lumen diameter, that tends to normalize WSS (Figure 9.5 upper part). Concomitant with a neointima formation, a paradoxal hypertrophy of the media develops. The reason for this medial growth response is currently unclear, but may involve teduced $\mathrm{NO}$ levels favouring VSMC growth.

\section{Flow-Induced Vasodilatation $\rightarrow$ Outward Hypertrophic Remodeling?}

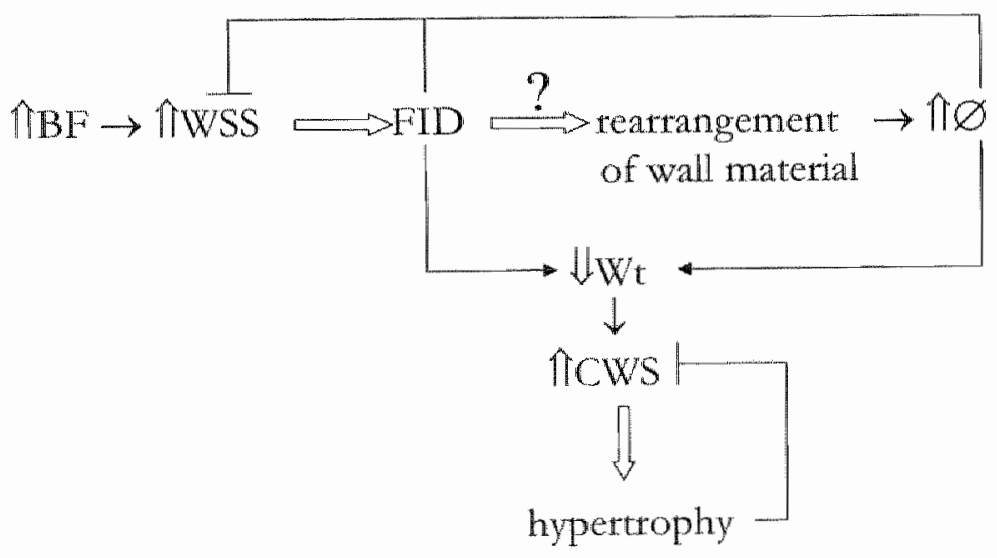

Figure 9.4. Sastaned fow-induced dilatation resulting in outward hypertrophic remodeling. Chronic wasodiatation caused by incensed wall shear stress will result in rearrangement of wall matcrial that leads to at structura humen diameter enlargement. This oumard remodeling will nomalize wall shear stress. Due to the decrease in wall thickness, circumferental wall stress increases, which triggers cell prolliferation sud/or smowth muscle cell hypertrophy. BF: blood Aow; WSS: wall shear stress; $\mathbb{H D D}$ : Пow-induced dilatation; $Q$ : structural diameter; W: wall thickness; CWS: circumferential wall stress; $\rightarrow$ : conseguence; open artow: strmulation; \{ increase; U. decrease, Legative feedback regulation. 


\section{Arterial Responses to Reduced Blood Flow (in small arteries).}

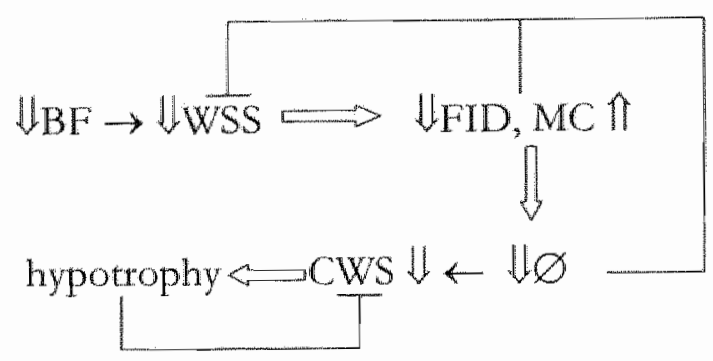

Strong BF decreases (in large arteries):

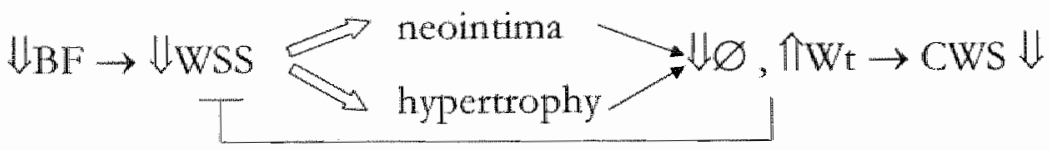

Pigure 9.5. Arterial ensponses to chionic blood flow reductons. Ophor part declines in blood fow will bower

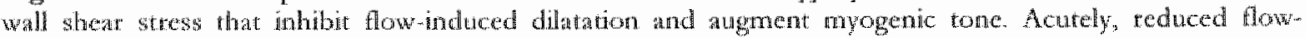
indaced diatation normalizes wally sheas stress, and chronically lead to structurn lumen narrowing (conpare ro pressureinduced inward tenodeling ats shown in Figute 9.2). This inward remodeling decreases circumferental wall wress and triggers hypotrophy of the arterial wall. Laymer part strong blood flow decreases result in necintima formation, either as a response to ingury or as part of an adapowe stategy to reduce lumen dianeite (iv order to nomalize wall shear stress) and to increase wall thickness (to nomalize circumferential wall stress). Abbreviations: BF: blood flow; WSS: wall shear stress, DID: flow-indaced diataton; MC: myogenic

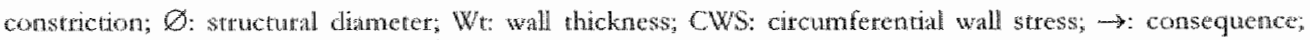

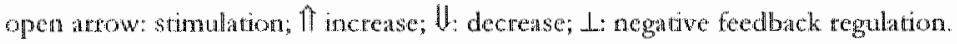

In genetal, arterial responses of small arteries to reduced blood flow lead to an inwatd hypotrophic remodeling (Figure 9.5 lower part). Reduced WSS shifts the balance berween IID and myogenic tone in favour of increased vasomotor tone, resulting in a structural lumen nartowing that decreases CWS, hereby inducing hypottophy. For instance, after giving birth, uterine blood flow decteases tapidly and is restored to nomal blood flow levels. Uterine arteries temodel their lumen diameter and wall mass in response to this blood flow decrease. In mice, uterine artery dianeter and wall mass retumed to nonpregnant values within 7 days postpartum. ${ }^{23,224} \mathrm{~A}$ few experimentally. induced models of chronic flow reductions have been applied to small arteries (Table 9.2). One of these involves a reduction of the workload of the tat cremaster muscle by unilateral orchidectomy leading to a treduction in blood flow to the cremaster muscle. After 3 weeks, significant inward hypotrophic remodeling of cremaster arterioles and inhibition of growth of arterioles in both size and number were observed. ${ }^{578}$ Furthermore, ligation of mesenteric resistance arteries in the rat and mice also leads to a rapid inward hypotrophic remodeling. $7,327,434,553,555,557$ VSMC contractility of these hypoperfused rat mesenteric resistance arteries was reduced, which was not solely due to the reduced wall mass. ${ }^{33}$ Like hyperperfused arteries, apoptosis and dedifferentiation of VSMC were 
involved in the structural response to chronic flow reductions. "In tho cultured rat mesenteric arteries have demonstrated that active tone (as chronic endothelin-1 vasoconstriction) is necessary to induce inward remodeling undet static pressurized conditions, because incubating vessels at low pressure $(2.5 \mathrm{mmHg})$ in the absence of tone did not result in permanent inward remodeling. ${ }^{17}$ This suggests that not decreased wall tension, but active tone is the trigget for inward remodeling. It remains to be elucidated whe ther also tone forms the driving force for low-induced inward remodeling.

\section{The Endothelium as a Flow Sensor}

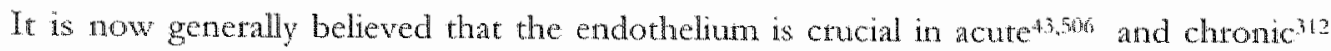
responses to changes in WSS, which is easily understandable since the endothelial cell monolayer is positioned at the interface between the vessel wall and the flowing blood. Evidence indicating a role of the endothelium as a shear stress sensor emerged from in witro studies of cultured monolayers.10,112 In response to sustained steady flows, endothelial cells align and elongate in the ditection of flow. 10,255 This process involves rapid deformation and reotganization of actin-containing stress fibers, as well as other cytoskeletal intermediate flaments at the sites of cell-matrix contact forming the focal adhesion complexes.25 The cytoskeleton is believed to play an important role in mechanosensing of fluid shear stress in whole vessels preparations. Shear stress induces rapid deformation of intemediate filaments. ${ }^{215}$ In arterial rings treated with cytochalusin D, phalloidin, or colchicine, flow-induced vasodilatation was attenuated, implicating the importance of microflaments and microtubules in flow-induced NO release. ${ }^{250}$ The intermediate filament protein wimentin is a component of the endothelial cytoskeleton. Vimentin-deficient mice show a blunted acute flow-induced dilatation of mesenteric resistance arteries ${ }^{20}$ and an impaired mechanical stability, migration and contractile capacity of fibroblasts. ${ }^{134}$ Uniateral left carotid artery ligation in vimentin knockout mice showed less maiked constrictive remodeling and mote pronounced medial hypertrophy of ligated arteries compared to ligated vessels of wild-type mice, whereas exaggerated ourward remodeling of the contralateral arteries was seen in vimentin-deficient mice compared to arteries of wild-type mice ${ }^{470}$ Similar overadaptive responses were observed in mesenteric arteries of desmin-deficient mice. ${ }^{327}$ So, the absence of one of these two main intermediate filaments lead to the same response (an cxcessive outward remodeling in response to chronic alterations in blood flow), despite blunted acure flow-induced dilatation, 20,327 indicaring that diameter adjustments in response to chronically altered blood flow can still proceed. This taises the question how shear stress induces long-tem remodeling in the absence of an apparently optimal mechanosensing. Since intemediate filaments are linked to the muclear lamina, shear stress may also effect the karyoskeleton. Through interactions between cell surface receptors and cytoplasmic and nuclear. intemediate filaments, ${ }^{20}$ shear stress may directly regulate gene expression independently of biochemical signaling mechanisms such as $\mathrm{NO}$ release. 215.243

In genetal, two levels of molecular signaling can be distingushed in response to shear stress. The first is the rapta activation and regulation of proteins or structures in response to acute changes in flow. One of the best studied shear stress-responsive proteins is endothelial nitric oxide synthase (e(c)NOS or NOS3). Stimulation via this 
pathway leads to a rapid increase in endothelial NO production leading to flow-induced vasodilatation. ${ }^{43,5016}$ Prostaglandines, ${ }^{36}$ bradykinin, ${ }^{37,235}$ and EDHF, ${ }^{24,246}$ as well as vasoconstrictors, such as endothelin- $1^{25}$ and reactive oxygen species can also be released in response to shear stress elevation. ${ }^{324}$ The second response involves activation of specific transcriptional regulatory elements termed shear stress-response elements (SSREs) in the promoters of shear-responsive genes resulting in the regulation of gene expression. SSREs have been identified in the promotor region of several endothelial expressed genes, such as PDGF-A $A^{48}$ and MCP-1. The The proteins are important in several pathophysiological processes of arterial remodeling, indicating that the endothelium not only senses and transduces shear stress, but also iniviates gene transcription and arterial temodeling. ${ }^{4(t)}$

Because mechanotransduction is only one aspect of flow-induced arterial remodeling, a detailed outline of the intraceliular molecular transduction signaling would be beyond the scope of this review, which focuses on structural alterations in the entire vessel wall. Therefore, the reader is referred to some excellent reviews on signal transduction $16,38,4 \%, 544$ and gene expression in response to fluid shear stress. ${ }^{542}$ In the next section we will discuss the role of endothelium-derived vasoactive mediators in more detail.

\section{Role of Endothelium-Derived Mediators}

Langille and ODonnell demonstrated that the typical inward remodeling that develops in response to the prolonged decrease in carotid artery blood flow due to ligation did not occur whon the endothelium had been removed.312 Other studies confirmed the importance of the presence of the endothelium during flow-related atterial remodeling. 264,539 The mechanisms by which the endothelium controls flow-induced remodeling are not fully understood. Langille and Bendeck addressed the role of prostanoids, endothelin, and the local RAS in constrictive flow-induced arterial remodeling. However, none of these mediators seemed to affect the remodeling, suggesting that a nowel vasoactive substance initiated the adlaptive response to reduced blocd flow. ${ }^{31}$ In this section we address the contribution of endothelium-derived factors on acute and long-rerm arterial diameter adjustments in response to altered blood flow in closer detril.

\section{Nimic Oxide}

Elevations in fluid shear stress increases the activiry and expression of eNOS in cultured endothelial cells, 113 whereas low or oscillatory shear stresses do the opposite. ${ }^{612}$ In wivo situations of increased blood flow have been shown to be accompanied by increased eNOS expression and NO release. $361,384,42,5,533$ direct role of $N O$ in arterial remodeling in who has not been firmly established. Suppression of NO synthesis by treating rabbits with nonhypertensive doses of the NOS inhibitor $N^{*}$-nitro-L-arginine methyl ester (L-NAME) inhibited flow-induced outward remodeling and failed to normalize WSS values in carorid arteries ${ }^{54}$ A similar observation was made in rats, ${ }^{201}$ suggesting that NO plays a role in the remodeling process in large arteries. However, studies in eNOS-deficient mice only 
showed a role for $\mathrm{NO}$ in constrictive remodeling, whereas the outwatd remodeling was comparable between eNOS-deficient mice and wild-rype mice ${ }^{463}$ An explanation for this discrepancy might be the extent of the fow increase in the two models. A threshold watue of WSS elevation to initiate remodeling has been postulated by Brownlee and Langille, since they observed that a $60 \%$ increase in BF did not cause any ourward remodeling two months after creating a left-to-right carotid anastomosis in adult rabbits. ${ }^{62,310}$ In the eNOS-deficient mice, the increase in carotid artery blood flow was only $50 \%$ (below the wheshold value of $60 \%$ ), compared to 3.2 to 7 -fold increase in blood thow after applying a lefi-to-right carotid anastomosis in adult rabbits.

The role of $\mathrm{NO}$ in flow-induced remodeling of resistance-sized arteries is controversial. Athough eNOS expression in small arteries is cortelated with WSS, 320,555 phamacological inhibition of ENOS by L-NAME does not prevent fow-related arterial and atteriolar remodeling. ${ }^{84}$ This suggest that other medrators than NO can play a role in flow-related arterial remodeling and can compensate for the loss of NO. Indeed, studies in eNOS-deficient mice have observed compensation for the loss of NO production. Acute wasodilatation in isolated skeletal muscle arterioles was mediated solely by EDFF in female eNOS deficient mice, ${ }^{2+4}$ whereas this was mediated by prostaglandins in male

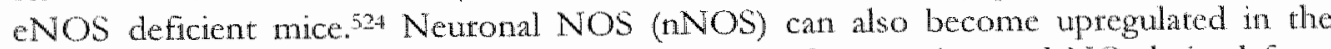
endothelium in coronary arteries of male $\mathrm{NOS}$ defictent mice, and NO detived from nNOS together with prostaglandines maintan flow-induced dilatation in these mice. 245 These data suggest gender and tissue specific compensation of the loss of one vasodilator and might explain why in some arteries, such as the carotid artety, chtonic pharmacological inhibition of NOS wili not be compensated by alternative endotheliumderved mediators, and in others, such as the mesentenc artery, upregulation by $\mathrm{EDHF}$ and/or prostaglandins can occur. However, whether this explains the difference in arteriall remodeling in response to chronic changes in blood flow remains to be established.

The unilateral carotid attery ligation method applied to mice deficient in $\mathbb{N O S}$ demonstrated a critical role for endogenous NO as a negative regulator of VSMC
proliferation in response to a decrease in blood flow 46 These mice displayed a hyperplastic response of the carotid wall and a grearer neointima formation compared to arteries of wild-type mice. In who gene unsfer of $\mathrm{NOS}$ has been shown to significanty inhibit neointima formation after balloon angioplasty, suggesting a vasculoprotective tole of eNOS in the remodeling of hypoperfused arteries. Neuronal NOS (nNOS) deficient mice also exhibited constrictive remodeling caused by flow disruption and accoletated neointima formation, suggesting that nWOS suppresses atherosclerotic lcsion formation in mo. .72 $^{\text {. }}$

Degradation and synthesis of extracellular matrix (ECM) proteins are vital processes during arterial remodeling. In order for vasculat cels to expand and migrate matrix reshaping is necessary. NO seems to partipate in MMP activation sth Flow-induced MMP activation is increased after fow-induced arterial enlatgement of the rabit carotid artery. ${ }^{5 \text { th }}$ A significant increase in MMP9 expression was measured in the ligated arteries that coincided with cell migration and the initial luminal enlargement ${ }^{18}$ suggesting that MMP activation is crucial in flow-induced arterial enlargement. Further evidence for MMPs to play an essential role in flow-induced remodeling comes from studies using tissue inhibitors of MMPs, ${ }^{3}$ targeted disfuption of MMP-9n3 and microarray analysis ${ }^{3}$ 
After unilateral carotid artery ligation, less intimal proliferation is observed and therefore a larger luminal area in MMP-9 deficicnt mice. Furthermore, absence of MMP-9 leads to increased collagen content, and thus increased stifness of the arrerial wall. ${ }^{73}$ Inhibirion of certain MMPs my have beneficial effects in that it leads to inward remodeling, hereby sttengthening the arterial wall and leading to mote stable atheroscletotic plaques. Excellent reviews are avajlable that describe the role of MMPs in vascular remodeling and atherogenesis in more detail. 172418

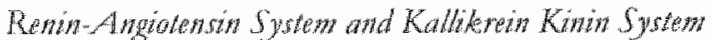

In vitro experiments have shown that Ang II induces growth responses in cultured rat carotid arteries, Also, mechanical forces such as pressure and flow, CWS and WSS respecrively, stimulated local synthesis of Ang $I$, which in turn induces protein synthesis expression of extracellular matrix proteins, such as fibronectin and collagen, wa the $A T_{1} R^{2022}$ These data imply that the trophic effects of Ang II are medated by angtorensin rype 1 receptor (AT, R) actions. The progression of neointimal thickening induced by Ang II was mediated wia $A T_{1} R s$, and not $A T_{2} R_{2}$, in injured rat catotid arteries. ${ }^{5}$

Chronic blood flow elevations created by placing a clip over the abdominal aoma, caused a reduction in wascular ACE activity by $40 \%$, suggesting that shear stress alters

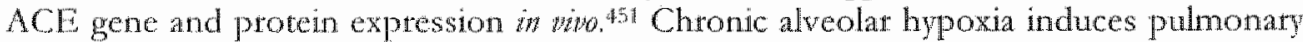
hypertension and hypertrophy of small muscular pulmonary arteries. 412 This pulmonary vascular remodcling is attenuated in tissue ACE-deficient mice, suggesting that local production of Ang II by ACE plays an important role in the pathogenesis of pulmonary vascular remodeling during chronic hypoxia. 50 Phatmacological inhibition of $A C E$ has been shown to suppress pulmonary vasculat neointimal fomation ${ }^{\text {tor }}$ and the role of $A C E$ inhibicors in the regression of the pressure-induced inward hypertrophic remodeling of resistance arteries has been firmly established (for a review see?2). Since ACE inhibitors also block the degradation of kinins, such as bradykinin, ${ }^{5}$ the beneficial effects of ACE inhibitors might be mediated by enhanced levels of kinins. Bradykinin modulates vascular tone by stimulating vascular endothelial $\mathrm{B}_{2}$ receptors and the subsequent release of $\mathrm{NO}$ and prostacyclin (PGI2) Bergaya and colleagues, demonstrated the involvement of kinins in acute FID of carotid arteries in studies involving tissue kallikrein cleficient mice. We observed that pregnancy-related uterine artery remodeling was normal despite blumed IID of uterine arteries in these same knockout mice ${ }^{223} \mathrm{ACE}$ inhibition matkedly reduced the neointimal formation after blood flow cessacion in mouse carotid atteries. This inhibition of intimal lyperplasia was significanty blunted after bradykinin receptor inhibtion. " Futhemore, gene transfer of human tissue kallikrein, the kinin-forming cnzyme in the endothetum, inhibited neointima formation after cessation of carotid artery blood flow in mice 137 These beneficial actions were medated va $\mathrm{B}_{2}$ receptor signaling. ${ }^{13}$ These resuls demonstrate a tole for kinins ifo suppressing VSMC growth and migration, cithet directly or inditectly via $\mathrm{NO}$, but do not show a tole for kinins in arterial lumen adjusments in tesponse to chronically altered blood flow.

\section{Enrothelus 1}

Cultured endothelial cells exposed to physiological levels of shear stress cause an early transient upregulation of ET-1 gene expression followed by a sustained suppression "3, 
ET-1 production is enhanced during elevated CWS, causing increased active tone in resistance arteties during hypertension, promoting VSMC growth and inward remodeling. ${ }^{375} \mathrm{ET}$ treceptor deficient mice developed increased neointima formation after cessation of carotid artery blood flow. ${ }^{381}$ Blockade of ET, and ETh receptors reduced the hypertrophic remodeling of rat arteries with expermentally-induced hypertension. ${ }^{32}$ This stimulatory effect of ET-1 on DNA synthesis by VSMCs is attributed to the activation of epidermal growth factor peceptor ${ }^{26 !}$ and suppression of endothelial cell apoptosis ${ }^{4 \%}$ These observations demonstrate that ET-1 exerts hts favounble inhibitory growth actions wia $E T_{1}$ receptors in response to reduced WSS, whereas it promotes growth pathways in response to increased CWS.

\section{Gronsth Fawhs}

In culture, endothelial cells exposed to shear stress respond by transient increases in growth factors such as platelet-derived growth factor (PDGF) A and $B^{238,339}, 40$ and basic fibroblast growth factor $(\mathrm{FGF}){ }^{471} \mathrm{PDGF}$ is a potent mitogen for $\mathrm{VSMC}$ in witw and promotes VSMC migration and prolifetation. ${ }^{26}$ In culture, PDGF-A and B chains can both be induced by shear stress ${ }^{238}$ and a shear-response element has been found in the promotor sequence of the PDGF-A and B genes.48 However during increased blood how PDGF-B is downegulated.3\% PDGF ligands are upregulated in response to blood How reductions ${ }^{368}$ and dowaregulated in tesponse to elevations in blood fow. 552

Basic FGF is an endothelium-derived autoctine growth factortit that has high binding affinity for the extracelluar matrix. It is upregulated in wo during flow elevations were it may account for the increased endothelial cell density and downegulated in response to flow reductions, where it might explain the decreased endothelial cell density 3 "Fluid shear stress also induces FGF2 mRNA expression, ${ }^{339}$ and its expression increases during flow-induced outward temodeling. ${ }^{503}$ Treatment with $\mathrm{FGF}$ antibodies significantly inhibited luminal narrowing after carotid artery ligation, 65 whereas no differences in remodeling were observed in FGF2 knockout mice compared to wild-type mice 52 Reasons for this discrepancy might be the difference in method used (cessarion versus residual blood flow) or compensation for the loss of FGF2 by other FGFs.

Transforming growth factor-beta (TGF 3 ) is a potent regulator of the cell cycle in smooth muscle and endothelial cels. $510.5 x+$ In culture, fluid shear stress induced condothelial TGL- $\beta 1$ production. ${ }^{400}$ TGF- $\beta 1$ and $\beta 3$ expression in the arta was associated with endothelial and VSMC proliferation which preceded the outward remodeling in thesc low-loaded aortas.

\section{Effect of Aging on Flow-Induced Arterial Remodeling}

From the above if is clear that alterations in flow-induced dilatation or production of endothelum-derwed vasoactive compounds has litele effect on chronic flow-related arterial remodeling. In shatp contrast, arteral remodeling seems to be very sensitive to age-related arterial changes. Aging progressively results in a reduced endothelial regulation. of vasomotor tone, $301,347,355$ a blunted reactivity of VSMC to vasoactive compounds, 54 differences in protein expression and changes in ECM rumover and composition, 
Furthemore, aging thas been shown to increase the level of reactive oxygen species such as superoxide anions and peroxynitrite. 103,562 These compounds interact with many signaling parhways that control the expression of adhesion molecules and those that regulate cell proliferation, trigtation and angiogenesis and which lead to a reduced

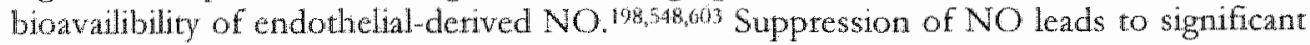
increases in ET-1 production. ${ }^{2}$. These processes create a milieu that predisposes to hypertension, atherosclerotic lesion formation and impaiment of flow-induced arterial remodeling. Studies of carotid artery remodeling in the rabbit ${ }^{31031}$ and rat have demonstrated blunting of flow-induced arterial remodeling with aging. Miyashiro and colleagues observed that juvenile tats experienced more outward remodeling in response to a $\pm 46 \%$ increase in carotid artery blood flow for a 4 -week period than in adult rars. ${ }^{3}$ Brownlee and Langille observed that a $60 \%$ increase in BF did not cause any outwat remodeling two months after creating a left-ro-right carotid anastomosis in adult rabbits, whereas significant outward remodeling in response to a $47 \%$ increase in blood flow was wisible in juwenile rats, 62 after which these authors proposed the "threshold hypothesis". This decreased ability in temodeling might result from a decreased capacity to sense WSS, which could elevate the threshold value to initiate a remodeling response.

Similar to large arteries, flow-loaded mesenteric arteries of mature rats display? absence of flow-induced luminal expansion, whereas a normal outward remodeling occurred in juvenile animals. 554 Medial hypertrophy was present in boch juvenile and matuse animals, suggesting that absence of luminal expansion does not entall lack of wall remodeling. In other words, WSS elevations were detected by the endothelium and the underlying signaling, resulting in endothelial and VSMC proliferation, is intact in mature animals. The absence of ourwatd remodeling of atteries of mature rats was not due to an altered eNOS expression profile compared to younger tats. ${ }^{554}$ This means that the shear stress-induced regulation of $\mathrm{NOS}$ expression is not impaired by aging and does not account for the impaired remodeling response.

\section{Conclusions}

Analogous to vasculat adaptations to pressure, arteries have strategies to adapt to longtem altorators in blood flow. Vasoactive mediators that are released by shear stress result in acure vasodiatation or vasoconstriction in order to notmalize wall shear stress. Chronic responses to aleted wall shen stress involve more long-tem molecular events such as genc and protein expression, extracellulat matrix re-scaffolding, cell proliferation and apoptosis, that ulrimately lead to wall restructuring that nomalize circumferential wall stress and wall shear stress. In this review we have addressed the issue wherher acute and chronic responses to WSS are associated with each other. A number of studies have studicd both acute and chronic responses of arteries to altered blood flow (Table 9.4). Snudies using animal models with reduced availability of rasotelaxing compounds or impaired mechanosensing have demonstrated that flow-induced arterial enlargement can still proced despite blunted acute flow-induced dilatation, suggesting that blunted acute vasomotot responses to WSS do not necessarily entail a defect in chronic wall restructuring. In an effort to explain these observations we have proposed several remodeling strategies of large and small arteties in response to altered blood flow. When 
flow-induced vasodilatation is unable to restore chronic wall shear stress walues, rearrangement of wall material leading to outward remodeling is initiated by these augmented stresses on the endothelium (Fyre 9.6). The exact mechanotransduction and biochemical signaling leading to this ourwand remodeling are not fully understood. Eithen other mediators are involved, or WSS is transmitted from the cell surface and cytoskeletal filaments to the nucleus, independent from mechano-chemical signaling processes, suggesting that acute and chronic tesponses to WSS can operate independenty from each othet as suggested by Maniotis and colleagues 34

In both small and large arteries, tesponses to chronic reductions in blood flow lead to inward remodeling. Predominately in large arteries, chronic large declines in blood flow promote neointimal growth, triggered as part of an adaptive strategy, that reduces lumen diameter and increases wall shear stress towards nomal values. Nitric oxide mediates its favourable actions in reducing the intimal growth via antiprolifetative and antinigratory effects on vascular smooth muscle cells.

The beneficial actions of ACE inhibition are indivecty related to nitric oxide (by thacreasing the half-life of bradykinin) and directly related in reducing the trophic actions of Ang II via $A T_{1}$ receptors. These actions of vasoactive mediators such as nitric oxide, bradykinin, Ang II, endothelin-1 and reactive oxygen species mostly affect arterial wall mass changes rather than arterial diameter adjustments.

Aging and absence or inhibition of cettain MMPs seem to be the only factors that cause defects in arterial diameter adjustments in response to altered blood flow. Arterial diamerer adaptations involve ECM re-scaffolding mediated by MMPs and cell migtation and cell turnover, that seem to be functional in situations of inappropriate endothelial function, suggesting that acute and chronic responses to altered wall shear stress can operate independently from ench other:

\section{Future Perspectives}

Fow-telated arterial remodeling has never been demonstrated in witro, strongly arguing that the interplay of endothelial cells and blood-born cells, such as monocytes and macrophages, is of importance in reshaping arteries in response to chronic alterations in blood flow. Therefore, much attention will be focussed on physiological and experimental setrings of flow-induced remodeling such as occurring during and after pregnancy and atteriovenous shunting. Much information will be extracted from micro-array and proteomics analysis. Given the fact that more and mote flow-related arterial remodeling is studied in mice, the use of genetically-altered mice will be of much bencfir in understanding the parhways that are involved in flow-induced atterial remodeling. This information is of help in the development of specific pharmacological inhibition and other therapeutic strategies for the reatment of cardiovascular discascs, including atherosclerosis, hypertension, diabetes, preeclampsia, wein graft discase, and restenosis after angioplasty. 
Twble 9.4. Modde studyng acure (vasodiataton) and chronc (outward remodelng) arteral adustmens in response to chevations in fow.

\begin{tabular}{|c|c|c|c|c|c|c|c|}
\hline Mediator & Model & Artery & Species & Vasodilatation & $\begin{array}{l}\text { BF } \\
\text { Change }\end{array}$ & $\begin{array}{l}\text { Outward } \\
\text { Remodeling }\end{array}$ & Reference \\
\hline Desmin & Ligationis & Mesconteric: & Mouse & reduced & notmal & normal & 327 \\
\hline Dystrophin & Ligation & Mesenteric & Motse & reduced & rednced & reduced & 326,328 \\
\hline Virnentios & Mone & Mesentetic & Mouse & reduced & nomal & $\mathrm{ND}$ & 220 \\
\hline Vimentian & Jusgation & Catotid & Mouse & $\mathrm{ND}$ & reduced & increased & 479 \\
\hline Aissue & pregnancy & Uterine & Mouse & normal & increased. & nomal & 224 \\
\hline $\begin{array}{l}\text { Tissule } \\
\text { Mablukrein }\end{array}$ & Worte & Cutrotid & Mouse & reduced & notmal & nomal & 223 \\
\hline $\begin{array}{l}\text { Tisatue } \\
\text { labliken }\end{array}$ & pregnancy & Uerine & Mouse: & reduced & incheased & normal & 223 \\
\hline MOS & Ligation & Carotid & Mousc & $\mathrm{ND}$ & $\mathrm{MD}$ & normal & 463 \\
\hline $\mathrm{NOS}(\mathrm{NO})$ & $\begin{array}{l}\text { Chronic } \\
\text { L-VAME }\end{array}$ & Carotid & Rabbit & ND & reduced & reduced & 547 \\
\hline MOS NOS & $\begin{array}{l}\text { Chronic: } \\
\text { 1.-NAME: }\end{array}$ & Meselateric & Rat & ND & reduced & notmal & 84 \\
\hline Unknown & Aging & Mesenteric & Rat & ND & normal & teduced & 554 \\
\hline Unknown & Aging: & Garotid & Rat & $\mathbb{N D}$ & notmal & reduced & 361 \\
\hline Unknown & Agirg & Garotid & Rabbit & $\mathrm{ND}$ & nommal & treduced & 62 \\
\hline Unknown & Aging & Untrine & Mouse & $\begin{array}{l}\text { nommil/toduced } \\
\text { NO telease }\end{array}$ & $\mathrm{ND}$ & reduced & Ch. 8 \\
\hline
\end{tabular}




\section{Flow-Induced Vasodilatation and Flow-Induced Ontwand Hypertrophic Remodeling.}

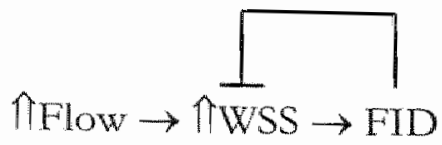

Strong BF increases:

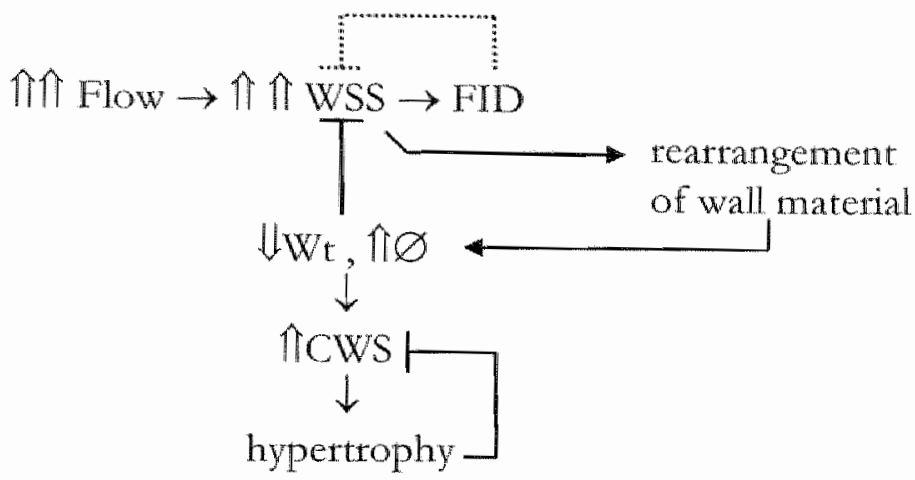

Figure 9.6. Flow-induced curward hypertrophic remodeling independent of flow-induced dilatation. Upyer prot: wall shear stress values can be normalized when the amplirude of the blood flow increase can be buffered by flow-induced dibation. Lower part however, when blood flow demand is large, such as duning pregnuncy in the urerine vascular bed, anothet strategy has to be tollowed. Wia as yet undefined mechanisms, latge increases in wall sheat stress can result dixectly in rearrangement of wall matcriat that enlarge the lumen diameter and nomalize wall shear stress. The decrease in wall thickness rises circumferendial wall stress that triggers cell

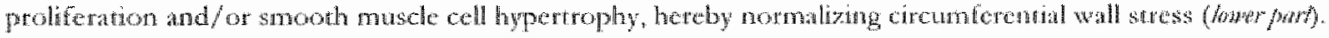

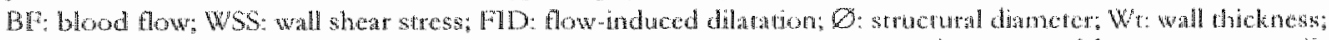

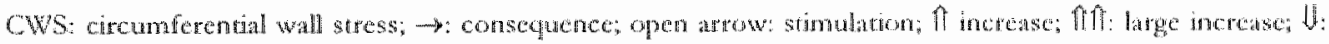
decrease; 1 negative feedback regralintion. 

Chapter 10

\section{General Discussion}


T'wo important hormonal systems, the renin-angiotensin system (RAS) and the kallikreinkinin system (KKS), which are regarded as opposing enzymatic strategies in blood pressure regulation, were subject of this thesis. A major determinant of the RAS is angiotensin-comverting enzyme (ACE), which can operate as a circulating enzyme and as an enzyme bound to the cell membrane, $A$ e tissue $A C E$ or $t-A C E$. A major determinant of the KKS is kallikrein, which functions as a circulating enzyme (plasma kallikrein) and as a rissue-boutid enzyme (tissue kalliktein, TK). The most important mediators of the RAS and $K K S S$ ate angiotensin II and bradykinin, respectively. In this thesis we evaluated the roles of these two enzymes and their effector peptides in acure and chronic tesponses of both small and large arteries to altered blood flow (shear stress). We studied these by using genetically-altered mice being deficient in either $-A C E$ or TK.

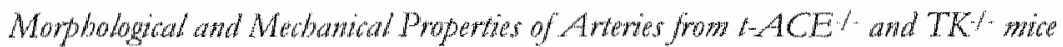

Catotid arteries of $\mathrm{TK} /$ mice were significantly smaller compared to $\mathrm{TK} / \mathrm{K}^{+/}$mice, whereas uterine artery structure was similar in both strains, suggesting that the $\mathrm{KKS}$ participates in the morphogenesis of large elastic arteries (chapter 3 and 4 ). The importance of $t-A C E$ for the maturation of arterial structure and elasticity was demonstrated in chapter 6 . Large and small arteries of $t-A C E / /$ mice exhibited a reduced arterial distensibility compared to wild-type mice. This increased arterial stiffness was not caused by an alteted fetal development, since chronic ACE inhibition (captoptill) duting postnatal development (staruing 3 weeks after birth until 12 weeks of age) decreased arterial distensibility in arteries from whld-type mice. We hypothesized that bradykinin $\mathrm{B}$. receptor stimulation might be involved in the regulation of atterial elasticity. After chronic (14-days) HOE 140 treatment, both the structural and mechanical changes in the $1-A C E-\%$ carotid artery were abolished, which suggests that the increased arterial stiffness in $t-A C E$. 7 mice was not related to the blood pressure, but rather involved $B_{2}$ receptors.

\section{Role of Angiotensin II and Bradyleinin in Ante Arterial Responstes to Shar Stress}

Bergaya and colleagues showed that carotid arteries of $T \mathrm{KK} / \mathrm{m}$ mice had a blunted shear stress-induced diatation, because of a lack of bradykinin generation by "TK in response to shear stress or fow ${ }^{3}$ Several studies have shown that angiotensin II crun lead to the production of bradykinin, $180,23,330,49,589$ that ovetexpression of angiotensin type $2\left(\mathrm{AT}_{3}\right)$ receptors can lead to increased bradykinin ptoduction, ${ }^{55}$ and that ATz receptors are involved in flow-induced vasodiatation. In chapter 2 , we showed that functional $\mathrm{AT}_{2}$ receptors, but not $A \mathrm{~T}_{1}$ receptors, play a key tole in flow-induced dilatation of catocid atteries. Furhermore, $B_{2}$ receptor antagonism in carotic arteties of wild-type mice had a comparable, non-additive effect, suggesting that these actions act in sequence rather than in synergy. Experiments by Katada and Maima provided evidence that the fomation of angiotensin 11 preceded that of bradykinin release. 23 These observations emphasize the interplay of the RAS and the KKS in the regulation of vasomotor tone, and hence arterial blood pressure, by counterbalancing, via endothelum-derived bradykinin formation, the powetful vasoconstrictor actions of angiotensin $I 1,563$ It is obvious that ACE, being the angiotensin $\Pi$-generating and bradykinin-degrading enzyme, plays a central role in these systems. In chapter 4 we confitm the key role of $\mathrm{TK}$ in flow-induced dilatation in resistance artertes (uterine artery). That bradykinin can also be released upon chemical 
stimulation (acetylcholine) was shown in chapter 5 , in which preconstricted uterine arterics of $t-A C E$ - mice have a blunted dilatation in response to acerlcholine when bradykinin $B_{2}$ receptors were blocked.

Mammalian ptegnancy is known to increase uterine arterial nitric oxide production.9891 Expression of endothelial nitic oxide synthase (eNOS) mRNA and WOS protein is increased during pregnancy, 3.57 probably because of estrogen- and vascular endothelial growth factor-induced upregulation of $\mathrm{NOS}$, 52155 hereby inctcasing uterine blood flow. Wh We observed an enhanced release of nitic oxide in uterine arterics of late pregnant wild-type and $\mathrm{TK} /$ mice (chapter 4 and 5). This upregulation was less obxious in uterine arteries of late pregnant t-ACE mice, where the contribution of bradykinin was more pronounced compared to wheir wild type counterparts, suggesting that bradylinin can compensate for nirric oxide during setrings of enhanced vasodilator activity (chapter 5).

Flow-induced dilatation was markedly reduced in uterine arteries of 7-days postpartum mice, despite normal acerylcholinemediated rasodilatation, suggesting a reduced shear stress sensing of the endothelium in these arteries (chapter and 5 ). We have curtently no mechanistic explanation for this plnenomenon, which mighe help restore the low uterine blood flow after pregnancy.

\section{Role of Angintensin II and Bradykinin in Chrowic Artewal Responser to Shear Stress}

The importance of TK and $t-A C E$ in atterial responses to chronic changes in atterial blood flow was investigated in chapters 3,4 and 7 . Chronic cessation of arterial blood flow resulted in a structural dectease in arterial lumen diameter (inward remodeling) in large conduit arteries and small muscular atteries, confuming numerous other studies. Carotid arteries of $\mathrm{TK} /$ mice showed a mote pronounced inward remodeling in response to a chronic cessation of carotid artery blood $\mathbb{A O W}$, whereas carotid anteries of $\mathrm{t}-\mathrm{ACE} ;$. showed a reduced inwand remodeling compared to their wild-type connterparts (chapter 7), suggesting a protective role of bradykinin in excessive inward remodeling. Inward remodeling was accompanied by a medial hypertrophy and VSMC hyperplasia in carotid arteries of wild-type, TK /, but not $\mathrm{t}$ ACE mice. No additional medial hypertiophy was observed in carotid arteries of TK mice. These observations suggest that not bratykin but angiotensin 11 , acting as a growth factor ${ }^{31,4 \text { th }}$ is responsible for the obscrved increases in medial wall mass and VSMC number.

In uterine aterics of 7 -days postpartum mice, which experience a drastic drop in uterine blood flow after deliwery, utetine arterial structure was indistinguishable from that of nonpregnant mice, indicating the dynamic nature of the uterine vascular bed (chapter 4 and 7). Reversal of uterine artery structure was comparable in wild-type, $T K$ and $-A C E$. - mice. Consequenty, intramural production of neither angiotensin 11 not bradyknin are essential for the adjustments of utertme artery structure to the mathed reduction in blood How occurring after pregnancy.

Arterial responses to chronic elevations in blood flow resulted in an outward eutrophic remodeling in carotid arteries of $-A C E /$ and $A C C^{*}+$ mice (chapter 7 ). However, no significant remodeling response was observed in carotid arteries ot $T K$. and TK ${ }^{+4}$ mice (chapter 3). The reason for this discrepancy most likely include stratiand age effects on the remodeling processes. ${ }^{2 m, 233,364}$ In chapters 4 and 7 we studicd the 
role of TK and $\mathrm{A}-\mathrm{ACE}$, respectively, in the pregnancy-selated changes of the urerine artery. We observed that $T K$ and $t-A C E$ deficiency did not compromise the outward hypertrophic temodeling of the uterine artery in response to the dramatic uterine blood flow inctease.

Overall, these observations indicate that intra-arterial production of angiotensin II contributes substantially to the hyperplastic temodeling of the ligated carotid artery but not to the pregmancy related uterine artery remodeling during and after pregnancy. Furthemote, they suggest a protective role for bradykinin in inward remodeling of large arteries in response to chronic reductions in blood flow.

\section{Maternal Endothelings and Posipaned Pregnaty}

The effects of postponed murine pregnancy on acute and chronic uterine artery responses to shear stress were investigated in chapter 8 . Endothelium-derved nitric oxide, which modulates arterial vasomotor tone and structure, is involved in the cardiovascular adaptarions to pregnancy. Estrogen and shear stress are known to stimulate eNOS phosphorylation. Our findings suggest that by midlife, the effects of shear stress on eNOS are lost, this is in line with human endothelial cells aged in cell culture, where shear stress failed to phosphorylate and upregulate eNOS.2\% Upregullation of alternative endotheliumderived relaxing factors such as prostacyclin and bradykinim compensates during acute flow-induced diatation, ${ }^{246,524}$ but can not sustain chronic pregnancy-telated wterine artety remodeling. This bunts the rise in blood flow during the third trimester of pregnancy, resulting in the observed fetal mortality.

\section{Acute Versur Clyomic Arterial Responses to Shear Sirers}

In chapter 9 we have addressed in detail the issue whether acute and chronic responses to sheat stress are interrelated. A number of studies have investigared both acute and chronic responses of arteries to altered blood flow. Overall, these studies suggest that blunted acute vasomotor responses to shear stress do not necessarily entail a defect in chronic wall restructuring. Chronic responses to atrered shear stress involve mote long-term moleculat events such as gene and protein expression, extracelluat matrix re-scaffolding, cell prolifetation and apoptosis. These processes ate most likely unaffected by defects in the groskeletal athitecture or in the acure relcase of endothelial-derived relaxing factots. However, defects in the phosphorytation of eNOS and the underlying signaling cascade 25 might be detrimental in the process of fow-related artertal remodeling.

\section{Perifutiven}

It may be woth considering that:

- bradykinin catn contributc to the consequences of (pharmacological) modulation of the RAS as is increasingly suggested by experimental and clinical drugintervention studies.

- flow-induced vasodilatation and-remodeling involve altemative pathways, which raises the possibility that valuable argets for cardiovascular pharmaco-therapy remain to be exploted. The key to these may be found in the unravelling of the effects of regionaliry and aging on arterial remodeling. 
- Adaptive arterial structural changes differ between pre-existing collateral arterics and large elastic arteries. This opens the possibility for site-selective interventions.

- Effects on arterial remodeling of even middle age are far more marked than those of blockade or deficiency of cardiovascular key enzymes such as 'TK and $t$ ACE. 



\section{References}

1. Aalkjar C, Haegery AM, Petersen KK, Swales JD, Mulvany MI. Exidence for uncreased media thickness, increased neuronal amine uptake, and depressed exciration-contraction coupling in isolated resistance wessels from essential hypertensives. Con Res. 1987,61:181-186.

2. Aartsen WM, Higers RHP, Schiffers PMH, Daemen MJAP. De Mey JGR, Smits JFM. Changes in wascular distensibility during angiotensim-converting enzymo inbibition involve bradykinin gpe 2 receptors. J Vase Res, 2004;41:18-27.

3. Abbruzzese TA, Guzman RJ, Martin RL, Yee C, Zarins CK, Dalman RL. Matrix metalloproteinase inhibition limis arterial enlargement in a todent arteriovenous tistula model. Swryeg. 1098; 124:328-335.

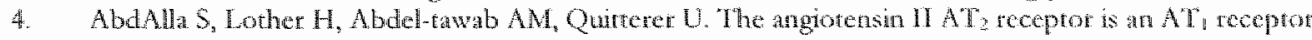
atatagonist. J Biol Chen. 2001;276:39721,-39726.

5. AbdAlla $S$, Lother $\mathrm{H}$, el Massiery A, Quiterer U. Incteased $\mathrm{AT}(1)$ receptor heterodincers in preclimpsita mediate enhanced angiotensin II responsiveness. Nat Med. 2001:7:1003-1009.

6. Achard JM, Foumier A, Mazouz $\mathbb{H}$, Caride VJ, Penar PL, Fenandez L.A. Protection against ischemia: a physiological function of the renin-angiotensin system, Biodien Ptyarmatiol, 2001;62:261-271.

7. Akiyama SK, Olden $\mathrm{K}$, Yamada KM. Fubronectin and integrins in invasion and meinstasis. Cancer Metastasis Rev. 1995; 14:173-189.

8. Alexandes RW, Brock TA, Grimbone MA Jr, Ritrenhouse SE. Angiotensin increases inositol triphosphate and calcium in vascular smooth muscle. Hyperteps:an. 1985.7.497.451.

9. Allen AM, Zhou J, Metidelsohn AO. Localization and function of angiotensin AT receptors. Mm d H)pertens. $2000 ; 13.31 \mathrm{~S}-38 \mathrm{~S}$.

10. Ando J, Tsuboi H. Korenaga $R$, Takada Y, Toyama-Sormachi $N$, Miyasaka M., Kanniya A. Shear stress inhilbits adhesion of cultured mouse endothelial cells to lymphocytes by downregulating VCAM-1 expression. Alm I Pathol. 1994;267:C679-C687.

11. Annibale DJ. Rosenfeld CR, Stull JY, Kamm KE. Protcin content and myosin light chrain phosphorylation in uterine arteries during pregnancy. Am J Pbycol. 1990;259:C484-C489.

12. Armstrong RB, Laughlin NF- Exercise blood flow patterns within and among tat muscles after uaining. Ans J Pbycrol. 1984,246; $\mathrm{H} 296-\mathrm{H} 306$.

13. Arras $M$, Ito WD, Scholz D, Winkler $B$, Schaper J, and Schaper W. Monocyte activation in angiogencsis and collateral growth in the rabbit hindlimb. I Chin Inwat 1998;101:40-50.

14. Ayajiki K, Kindermann M, Hecker M, Fleming I, Busse R. Intracellular pH and tyrosine phosphorylation but not calcium determine shear stress-induced nitric oxide production in native endothelial cells. Cirs Res. 1996:78:750-758.

15. Baffout R, Berman J, Garb JI, Rhee SW, Kanfman J, Friedman P. Enhanced angiogenesis and growth of collaterals by in vivo administration of recombinant basic fibroblast growe factor in a mbbir model of

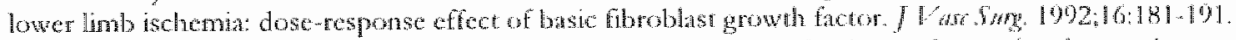

16. Bakker EN, Van der Meulen ET, Span JA, Van Bavel E. Organoid culture of cantulated rat resistance

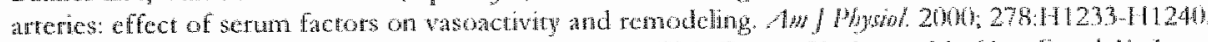

17. Pakker ENTP, Van der Meulen ET, Van den Berg BM, Everts Y, Span JA, Van Bavel L. Inward

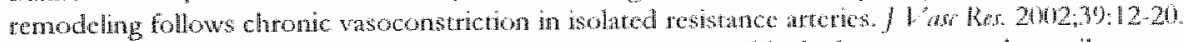

18. Bao $G$, Gohlke $P$, Qadri $F$, Unger T. Chronic kinin receptar blockate attenuates the anthypertensive effect of ramipril Hythtarion. 1992:20:74-79.

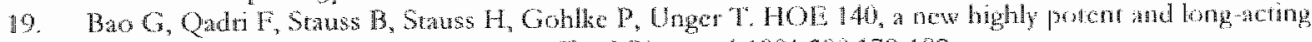
bradykinin antagonist in conscious rats. Ear / Phanyurol. 1991:200;17\% 182.

20. Bardy $N_{n}$ Karillon $G$. Merval $R$, Samuel J-I, Tedgui A. Differential effects of preseure and fllow an DNA and protein synthesis and on fobroncctin expression by arteries in a novel orgat culture system. Girm Res. 1995;77:684-694.

21. Bardy N, Merval R, Benessiano J, Samuel JI, Tedgui A. Pressurc and angiotensin II synergisnically induce aortic fibronectin expression in organ calue model of rabbit acmat. Evidence for a pressere-induced tissure tenin-angiotensin system. Cirr Re.5. 1996;79:70-78.

22. Batdy $\mathbb{N}$, Merwal $R$, Samuel $J L$, Tedguil $A$. Effecs of angiotensin II, pressure and flowe on fobronectin

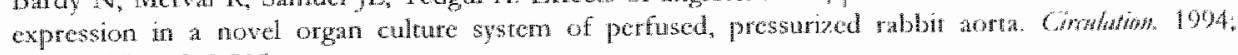
90 (suppl I, pt 2):1.515.

23. Barker DI. Fenal origins of coronary heart disease. BMJ. 1905:31:171-174. 


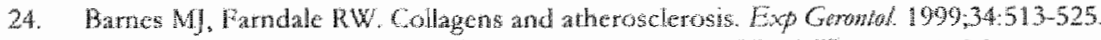

25. Barone PC, Knudsen DJ. Nelson AH, [etesten GZ, Whete RN. Mouse smain diferences in

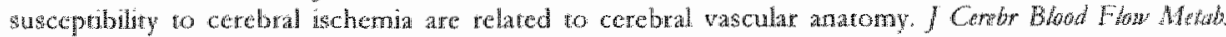
$1903 ; 1,683962$.

26. Barreto-Chaves ML, Ancas $\mathbb{I}$, Kricger $\mathrm{Z}$. Glucocorticoid regulaton of angiotensin-converting enzyme in

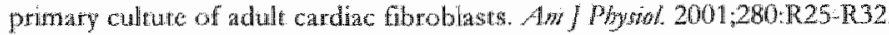

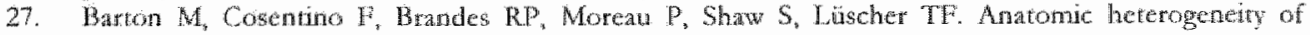

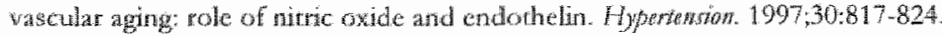

28. Burton M, Hadenshild $C \mathrm{C}$, DUScio JW, Shaw S, Manster K, Luscher TF. Endothelin ET, receptor blockade restores NO-mediated endothellat function and inhibits atherosclerosis in apolipopnotein deficiont mice Proc Nat Arad fri. 199895:14367-14372.

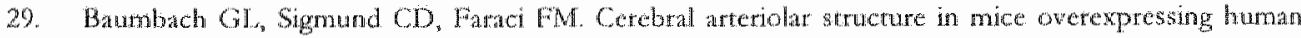

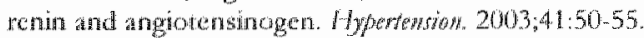

30. Bersely D, Schwart JH, Brenner BM. Interleukin induces prolonged Larginine-dependen aych guanosfnc monophophate and nitite producton in rat wascular smooth muscle cells. I Chn loves? 1991:87:602-608.

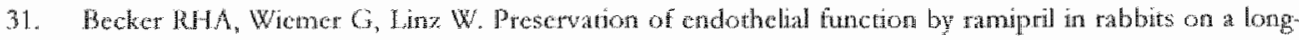

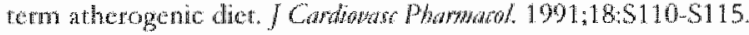

32. Bendeck MP. Langillc BI. Rapid accumulation of elastin and collagren in the aorts of she ep in the intermediane perinat poriod. Com Ro 1991;69:1165-1169.

33. Ben Driss A, Benessino J, Pottevin P, Lewy B I, Nichel JB. Arteral expanswe remodeling induced by high flow ancs. Am. I. Pbyrin. 1997,272:1851-H858.

34. Ben Driss A, Himber C, Poitevin P, Duriez M, Michel JB, Lexy BI, Enalapri improves arterial elastic

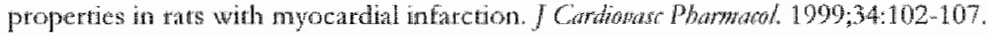

35. Benetos A, Guvas H, Stcwart JM. Vavej W, Hatinoglou S, Gavas I. Vasodcpressor role of endogenous

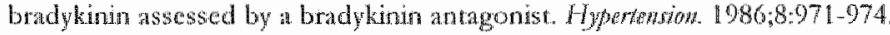

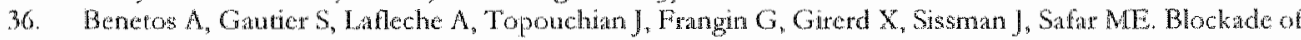

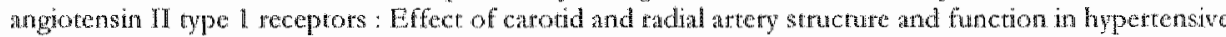
humansis / Var Rer. 2000;37:8-15.

37. Bergaya 5 , Mencton P, Bloch-Fure M, Mathien E, Ahenc-Gelas F , Levy BI, Boulanger CM. Decrensed

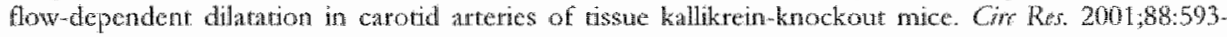
599

38. Bergel DH. The stac elastic properties of the arterial wall. Pbyto. 1961;156:445-457.

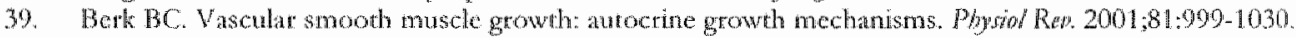

40. Berk BC, Rao GN. Angiocensin IHinduced vasculat smooth muscle cell hypertrophy: PDGF A-chaim mediates the increase in cell size. Coh Pbyrot. 1993; $154 \times 368-380$.

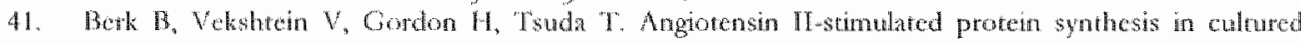

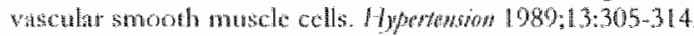

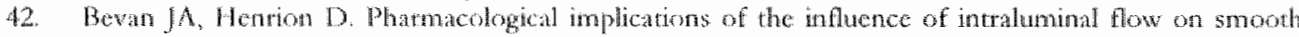

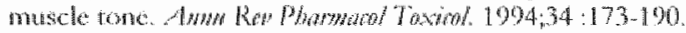

43. Bevon JA, Laher I. Pessure and Tow -dependene vacular tone. WASEB J. 1991,5:2267-2273.

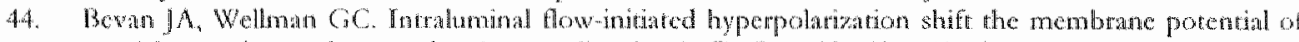

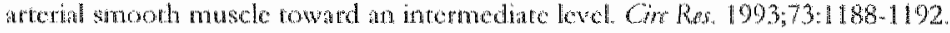

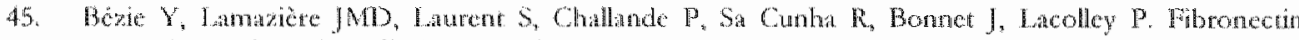

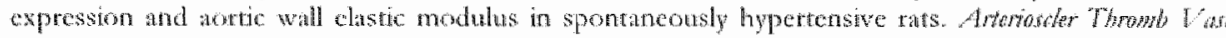
Bid. $1098,18: 1027-1034$

46. Bhagylakhmi A, Frangos J. Mothusm of shearinduced prostacyclin producton in zadothelial cels.

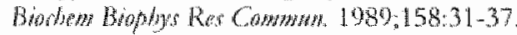

47. Banco $A$, Stonc J, Lymch L, l.apinski $\mathbb{R}$, Berkwwitz G, Berkowitz. RL. Pregnancy outcome at age 40 and

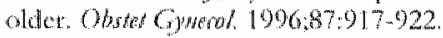

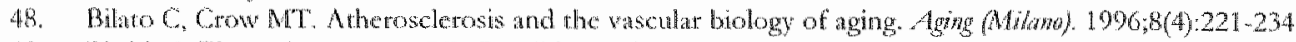

49. Bird MM, 2hang I, Magness RR. Possible mectanisms undelying pregrancy-induced changes in uretine

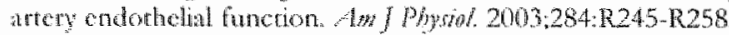

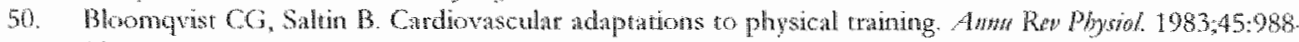
092. 
51. Blüher M, Kahn BB, Kahn RC. Exrended tongevity in mice lacking the insulin receptor in adipose trasue. Swente. 2003;299:572-574.

52. Boegehold MA. Heterogeneivy of endothelial function within the circulation. Carr Opm Nophm Hopertan. $1098 ; 7: 71-78$.

53. Bohlen HG. Arteriolar closure mediated by hypertesponsiveness to norepinephine in hypertensive rats. Als I Pbysial 1979,236:H157-H164.

54. Boo YC, Jo H. Flow-dependent regulation of endothelial nitric oxide synthase: role of protein thinases. Am I Pbysiol 2003;285:C499-C508.

55. Boonen HC, Daemen MJ, Eerdmans 1PH, Fazi GE, van Kleef EM, Schiffers PM, De Mey JO

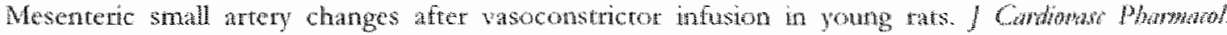
$1993: 22: 388-395$

56. Borell U, Fernstrom 1, Westran H. Hormonal infuences in the urerine arteries: an arteriographic study

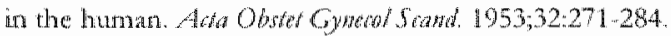

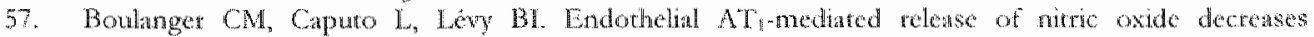
atagiotensin 11 contractions in sat carotid artery. Hypersensian 1995;26:752-757.

58. Brayden JE, Nelson MT. Regulation of arterial tone by acrivation of calciun-dependant potaseium channels. Saktat $1992,2565332-535$.

59. Brosens I, Robertson W, Dixon H. The physiological tesponse of the vessels of the piacental bed to normal pregnancy. J Path Bawt 1967; $93: 569-579$.

60. Brown NJ, Gainer JV, Murphey L), Vaughan DE. Bradykinin stimulates plasminogen actixator releasc from human foream vasculature through $\mathrm{B}_{2}$ receptor-dependent, NO syndhoce-independent, and cyclooxygenasewindependent pathway. Comblation. 2000;102:2190-2196.

61. Brown NJ, Gainer JV, Stcin CM, Vaughan DE. Btadykinin stimulates tissue plasminogen activaror relcase in human vasculature. Hyperfensioy. 1999;33:1431-1435.

62. Brownlee RD, Langille BL. Arterial adaptations to alterd blood flow. Can I Phyol Phormatiol. $1991,69: 978-983$.

63. Bruce NW. The effect on fetal dewelopment and ateroplacental blood tow of ligating a nterine artery in the tat near term. Teratolog: 1977;16:327-332.

64. 13 ruce NW, Giblas CP. Proceedings: The maternal placental circulation in the conscious abbit. f Reprod Feril. 1974;36:478.

65. Bryant SR, Bjercke RJ, Erichsen DA, Rege $A$, Lindner $V$. Vasculat remodeling in response to altered blood flow is mediated by fibroblast growth factor-2. Com Res. 1999;84:323-328.

66. Buches. B, Travo P, Maurent P, Stoclet JC. Vascuhar smooth muscle cell hypertrophy during maturation in rat thoncic aorta. Cell Biol Lat Rap. 1982;6883-892.

67. Butridge $K$, Fath K, Kelly $T$, Nuckells $G$, Turner CE. Focal adhesions: Transmembrane junctions

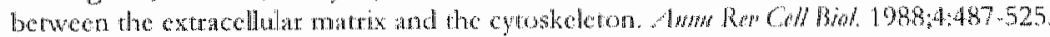

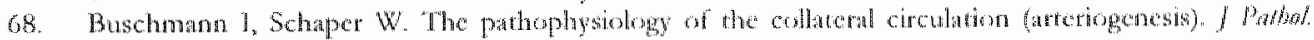
$2000,190: 338-342$

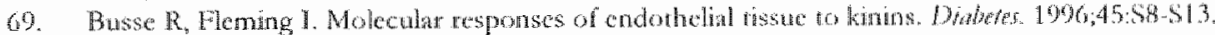

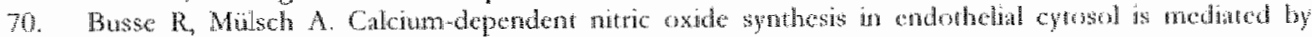
Culmodulin. FLBS Lett 1900,265:133136.

71. Buus CL, Pourageaud F, Fazzi GE, Janssen G, Mulwany MJ, Do Mcy JGR Smooth muscle coll changes

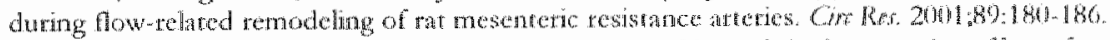

72. Cachefeiro $V$, Salkakiban T, Nasjetti $\Lambda$. Kinins, nitric oxide, and the bypotensive effect of captopin and Famiprilat in hypertension. Hypertextow. 1992;19:138-145.

73. Cai WI, Kolleai S, Koesis E, Scholz D, Kostin S, Lue X, Schaper W, and Schaper J Remudeling of the adventitia during cotonary arreriogenesis. Am / Physiol. 2003:284:H31-H40.

74. Cai WJ, Koltai S, Kocsis E, Scholz D, Schaper W, Schaper J Conncxin 37, nor Cs40 and CX4.3, is

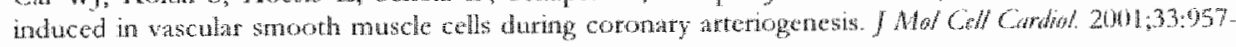
907 .

75. Cai W, Vosschulte R, Afsah-Hediri A, Koltai S, Kocsis E, Scholz D, Kastin S, Schaper W, Schaper J. Arered balance berwen extracelluar proteolysis and andipoteolysis is associated with adaptive coronary arteriogenesis. I Mol Coll Cardial 2000,22097-1011.

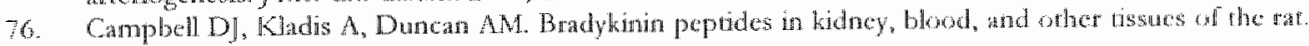
Hyportenion. 1993;21:155-165. 


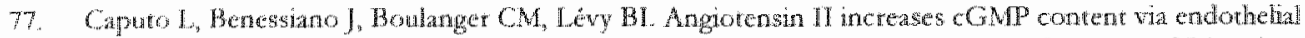

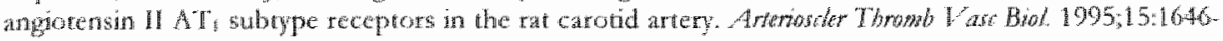
1651 .

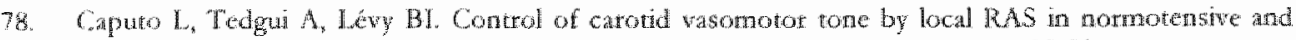

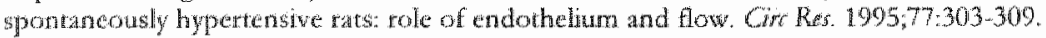

79. Carmelie P. Mechanisms of angiogenesis and arterogenesis. Wature Medicite 2000;6:389-395.

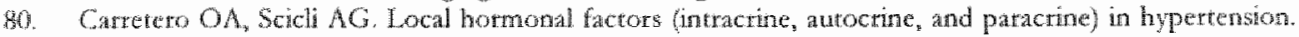
Typewation. $1991 ; 18158-169$

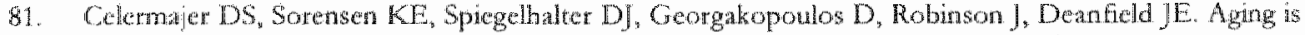

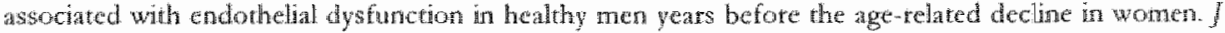
An Coll Combol 1994:24:471-476.

82. Charchat IJ, Kapuscink M, Harrap SB. Persistent reducton in renal nerve growth factor mRNA arter

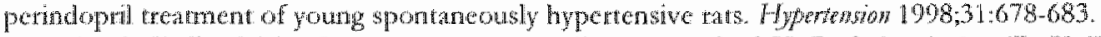

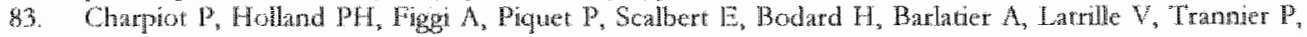
Mercier $C$, Luccioni $R$, Galat $R_{0}$ Garçon D. ACE inhibition with perindopri and atherogemesis-induced

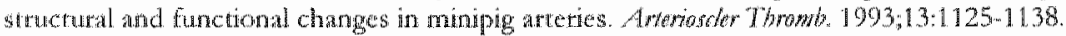

84. Celle, DL, De Mey JGR. Chwomic NGmito-h-argine methyl ester treamen does not prevent low

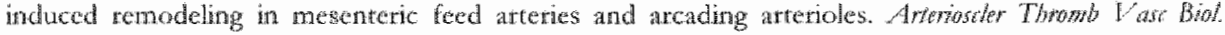
$2000: 20: 2057-2063$

85. Coiler DL, Nelissen Vranken HJMG, Smits JWM, De Mey JGR Presstate but not angiotensin Illinduced

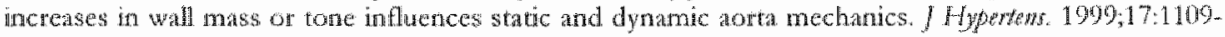
1116.

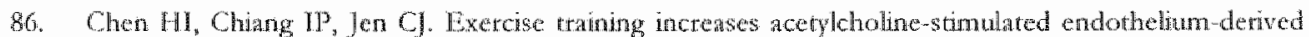

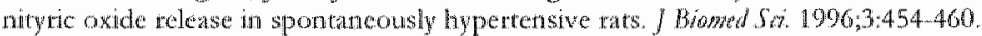

87. Chen HI, LA HT. Physical conditioning can modulate endotheliam-dependent vasorelawation in abbirs. Amerianis Thow V ar Biol. 1993,13.852-856.

88. Chen L, Xin X, Echart AD, Yang N, Fulet JE. Regulation of vasculat smooth muscle growth by alpha 1-adrenoreceptor subtypes in vitro and in situ. f Biol Cbem. 1995.270:30980-30988.

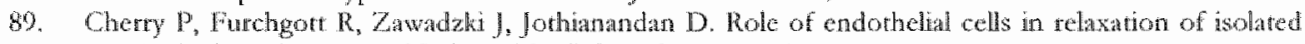

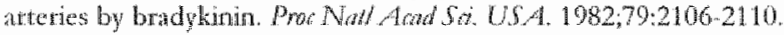

90. Cho A, Nitchel L, Koopmans D, Langille BI. Effects of changes in blood fow take on cell death and

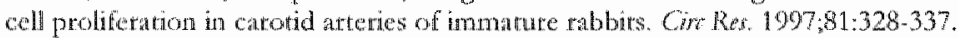

91. Christen T, Bochaton-Pialla ML, Meuvile P, Rensen S, Redat M, Van Eys G, Gabbini G. Cultured potcine cononary artery smooth nuscle cells. A new model with advanced differentiation. Cir Re. $1909,85,909107$

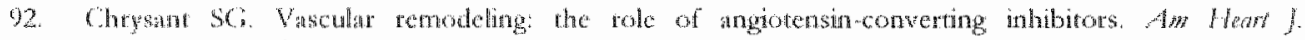
$1998 ; 135: 921-830$

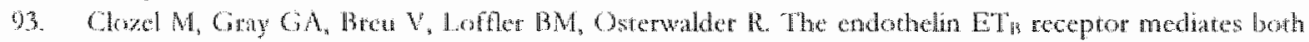

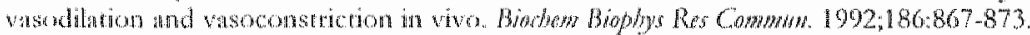

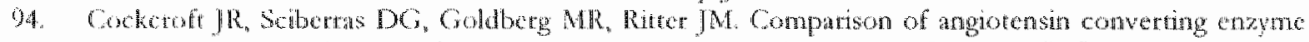

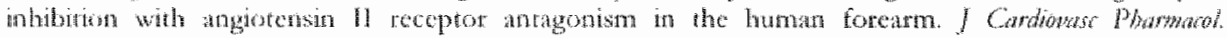
$109322579-584$

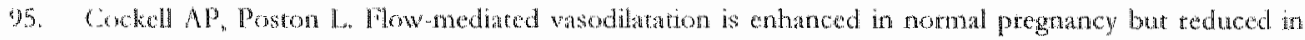
precelumpsit. Hyptemisn. $1997,30: 247-251$

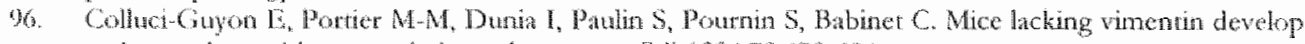

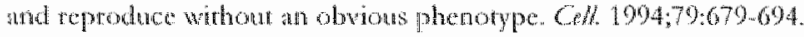

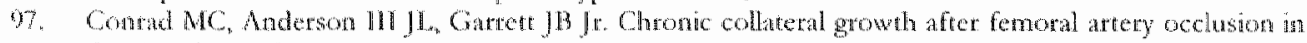
the dog / App/ Photiol. 1971:31:550-555.

98. Conmed KP Verier KA. Plasma level, winary excrion, and metabolic production of coMP during

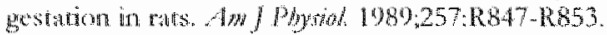

99. Cokk ol Davidge ST. Pregnancy induced alteratons of vasculat function in mouse mesenteric and

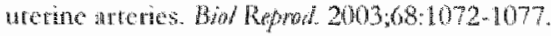

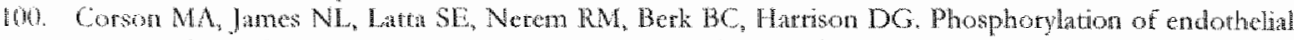
nicre oxide symthe in response to fuid shear stress. Corr Rer 199679084-991". 


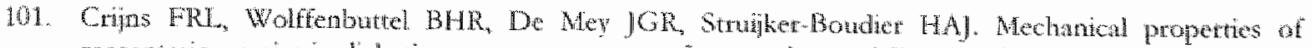

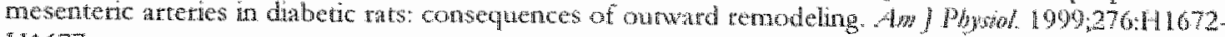
11677 .

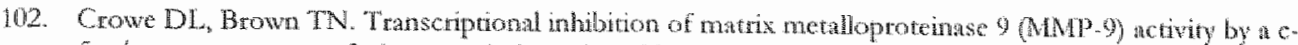
fos/estroger receptor whion proten is mediated by the promal $A$ - 1 ste of the MMP-9 promoter and

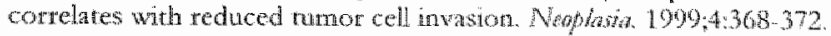

103. Csiszar A, Ungrat Z, Edwards JG, Kaminki P, Wolin MS, Kolter A, Kaley G. Aging indnced

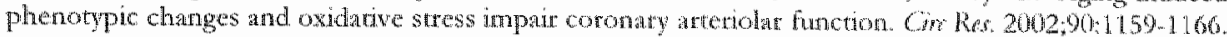

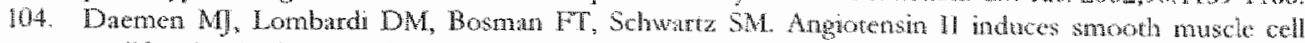

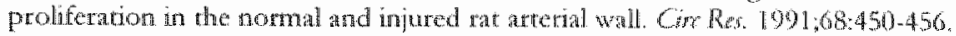

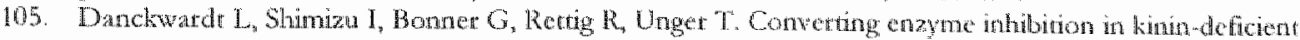
brown Notway ats. Hyowemon $1900,16429-435$

106. D'Angelo I.S. Shear stress induces chatges in the monphology and cyroskeleton organization of auterial

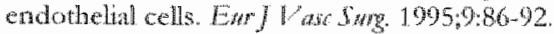

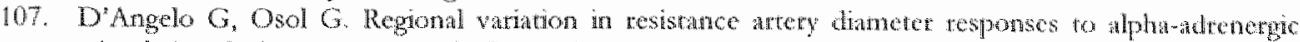

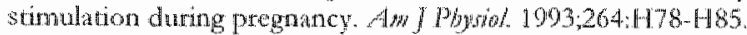

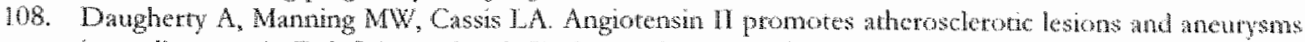

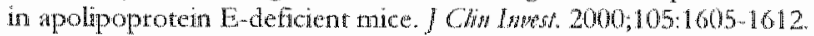

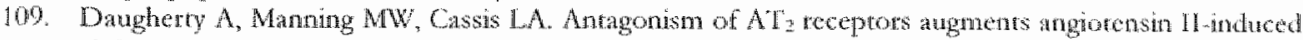
abdominal nothe anewrysms and atherosclerosis. Br / Pbarmat. 2001,134865-870.

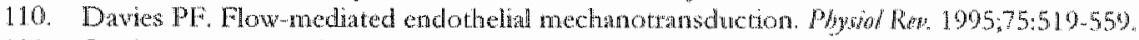

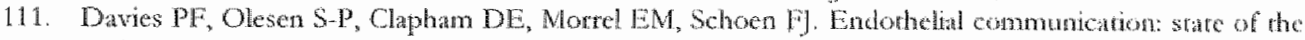
art lecture. Itpentomion. 1988; 1:563-572.

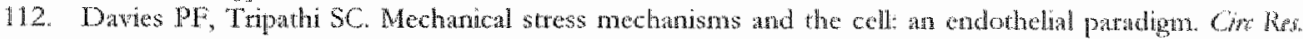
$8993,72,239245$

14.3. Davis ME, Cai H, Drumnond GR, Martson DG. Shea stess regulates endotheliat nituc oxide synthase expression through c.sinc by divergen signaling pathways. Cor Rer. 2001:80:1073-1080

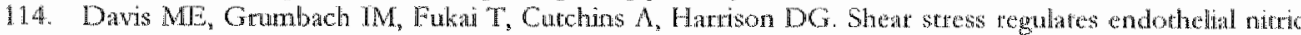

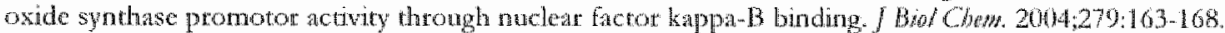

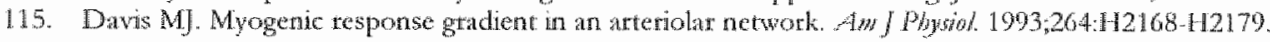

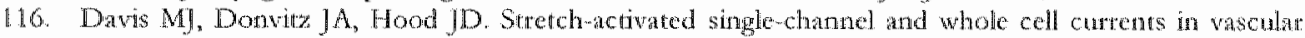
snooth muscle Am Pbsiof 1092;262:C1083-C1088.

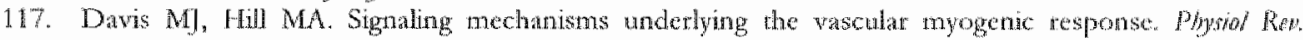
$1909 ; 70.387 \times 423$

118. Davis MJ, Meininger GA, Zawein DC. Streth-induced incteases in intracellular calcium of isolated

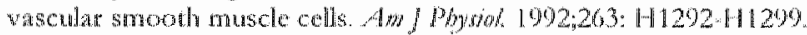

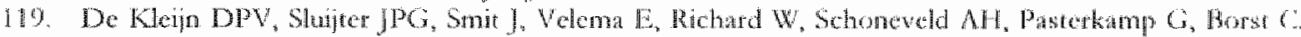

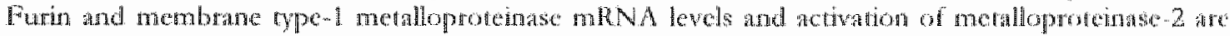

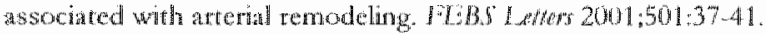

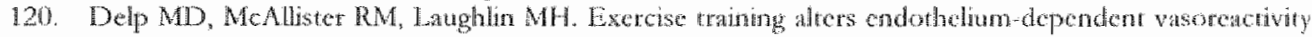

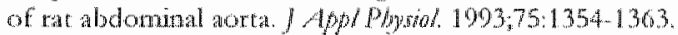

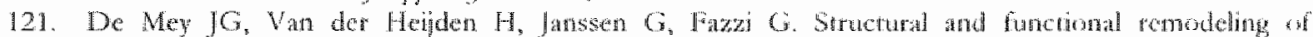

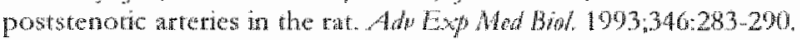

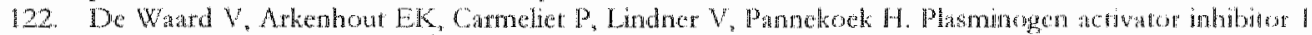

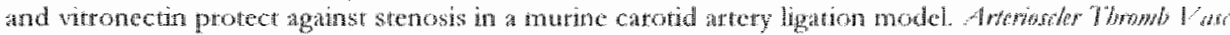
Brol $200222: 1978-1983$

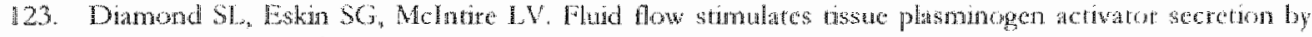
cultured human conothelal cells. Swene. $1989,243: 1483-1485$

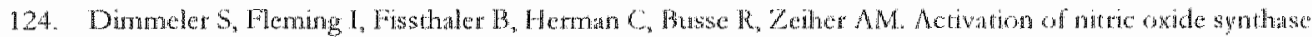

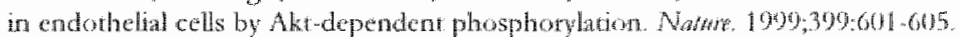

125. Dmmelet S, Rppmann $V$, Wetand U, Handelet, Zether AM Angorensin II induces spopiosis of

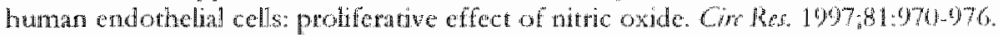

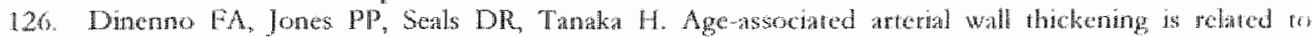

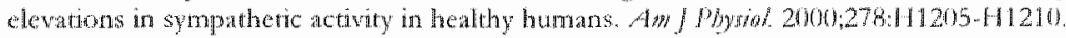




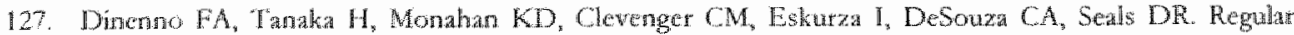

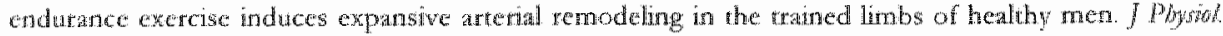
$2(001,534,28729) 5$

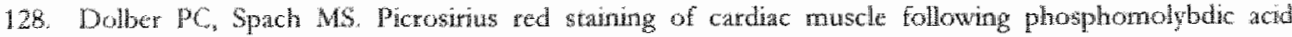

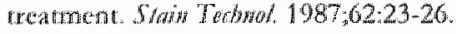

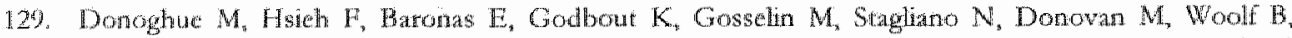

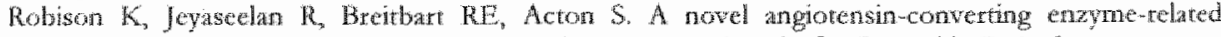

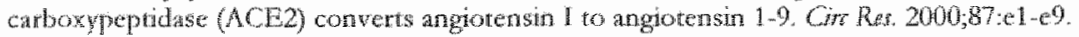

130. Dull RO, Dawes PF. Flow modulation of agonist (ATP) response $\left(C_{2}^{2}\right)$ coupling in vasular cndohelial cellis. Am / phyzol 1991:261:H149-H156.

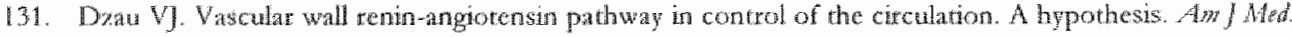
$1984,77: 31-36$

132. Duan V. Circulating vetsus local renin-angiotensin system in catdiovasular homeostasis. Ciradatow $1088,77.4-12$

133. Easterling TL Beredett T], Schmucker BC, Catson K. Milard SP. Matemal hemodynamics and aortic diameter in nomal and hyperensive pregnancies. Obwe Growe/. 1901,78:1073m1077

134. Eckes B, Drogic D, Colucci-Guyon $\mathbb{E}$, Wang $\mathbb{N}$, Maniotis A, Ingber D. Merckling A, Latugz $\mathrm{F}$, Aumailley $M$, Delowee $A$, Kotehanky $\forall$, Babinet $C$, Krieg $\mathrm{T}$. Inpaired methanical stability, migration and

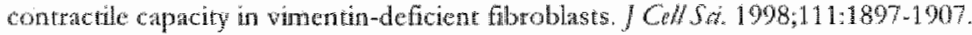

135. Egashira K, Inou T, Timook $Y$, Kai H, Sugimach M, Suzuki S, Kuga T, Urabe $\mathrm{Y}$, Takeshita A. EFtects of age on endothelium-dependent vasodihaton of resistance coronary attery by acetycholine in humans. Gimathon. $1993,88: 77,81$

130. Eganhat K, Koyanagi M, Kitamoio S, Ni W, Kataoka C, Morishita R, Kaneda Y, Akiyama C, Nishida K, Sucishi K, Takesha A. Antimonocyte chomoatractant protein-1 gene therapy indubits vascular

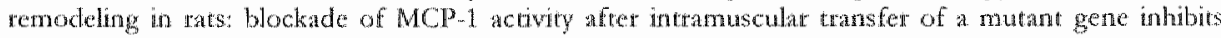

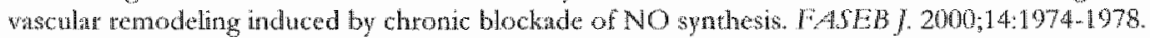

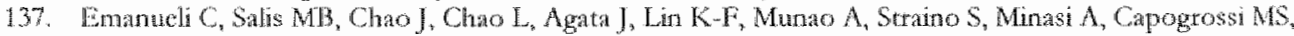
Madeddu P. Adenowirusimediated human tissue kallikein gene deliwery inhibits neointima tomation

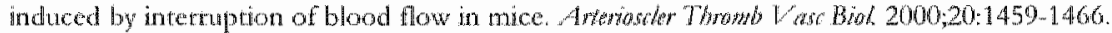

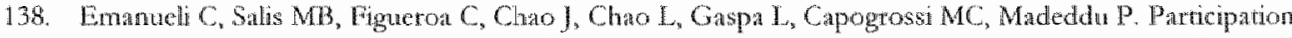

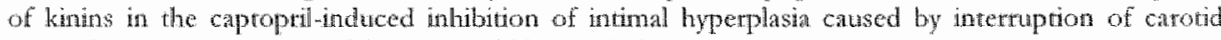
blood flow in the mouse, Bry Phamat $2000,130: 1076-1082$.

139. Erdos $\mathrm{EG}$. Angotensin 1 conwerting enzyme and rhe changes in out concepts though the years: Lewis K. Dathl memorial lecture. Hyomatuton. 1990;16:363-370.

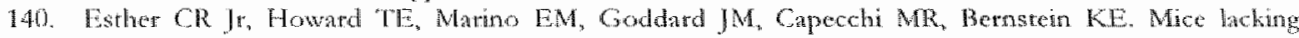

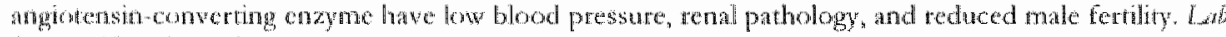
Inwe $19967.7453-965$.

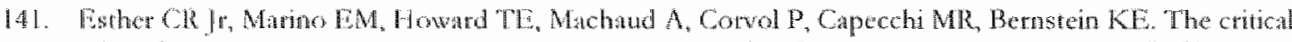

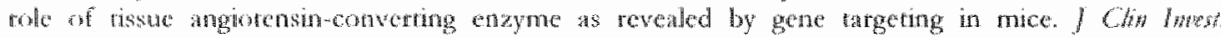
$1997,902375-2385$

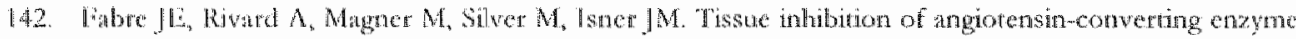

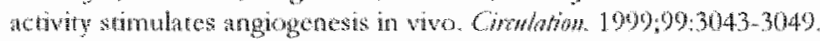

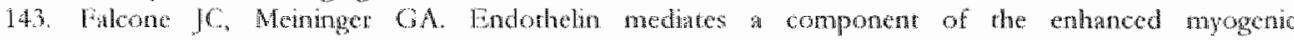

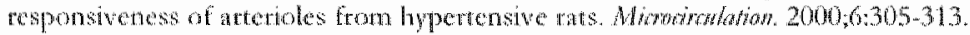

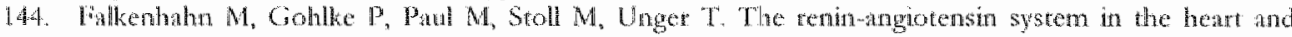

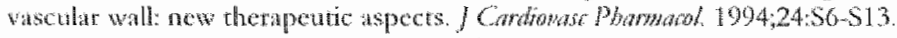

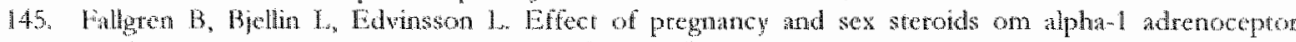

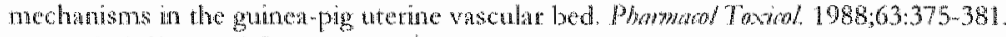

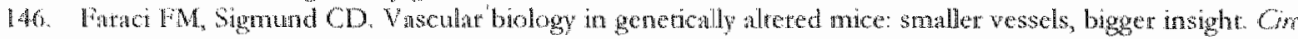
Re. $19990,85: 1214-1225$.

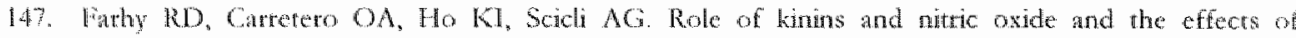
angictensin-comering enyme inhbitors on neointima formation. Cir Rei 1993,72:1202-1210.

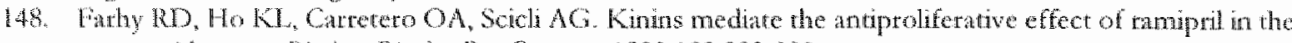

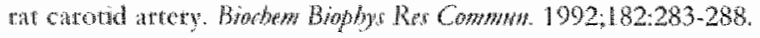


44. Fermandez-Alfonso MS, Marroma PA, Licka I, van Even P, Thobis D, Selwhens BA, Pal M. Warly

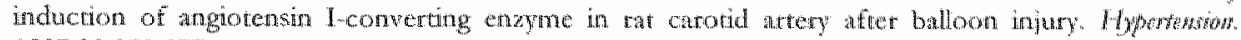
$1997,30.272277$.

150. Fertario CM, Chappell MC, Dean RH, Iyer SN. Nowel angotensin peptides raghate blood prossure.

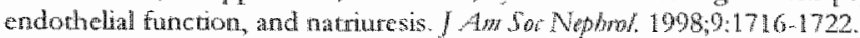

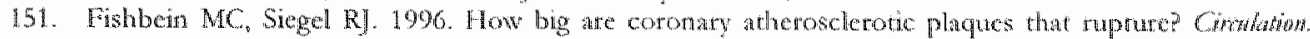
$1996,94: 2662-2666$

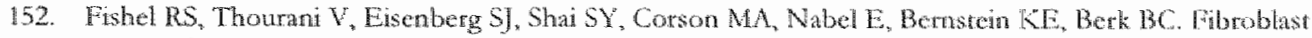
growh factor stmulates angiotensin converting erryme expression in rascular smood muscle cells.

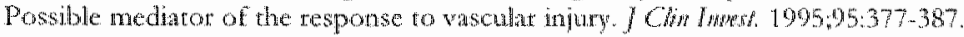

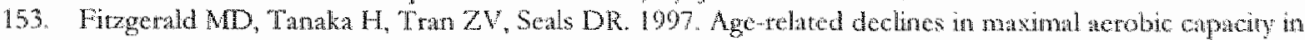

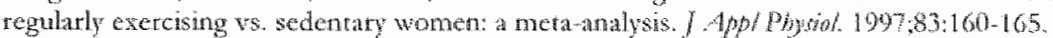

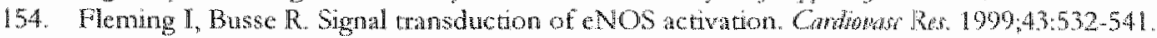

155. Folkow B. A study ot the facrors inthuche the tome of denervaced blood vessels pertomed at warous pressures. Alat Phy rol Sami 1952;27,99.117.

156. Folkow B. Description of the myogenic hypothesis. Cir Re 14-15, 1279-1295, 1964.

157. Folkow 13. Myogenic mechanisms the control of systemic tesistatre. Introduction and historicit

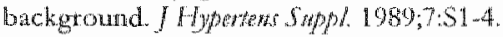

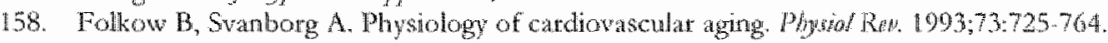

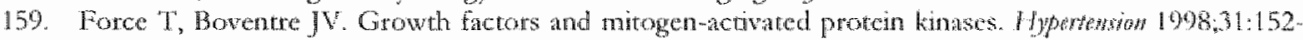
161.

160. Ford SP, Control of werine and ovarian blood tow throughont the cscous cycte and pregnancy of the ewe, sow, and cow. $7.4 n m .5 \%$. 1979;4\%:1510-1516.

161. Frangos JA, Fskin SG, McIntise LV, Wes CD. Dlow affects on prostacyclin protuction by culumed human endothelial cells. Srime $1985,227: 1477-1479$.

162. Frankin BA, Kahn JK. Delayed progression of coronary atherosclerosis with intensiwe wisk facto modification: effects of diet, drugs, and excevise. Sport Med 1996,22:306 320.

163. Fretrs RC, Schmitdid J. Mclean $\mathrm{FH}$, Usher RH, Goldman MB. Inctemsed naternal age and the risk of feral death. N Eagl Med. 1995,333:253-957.

1.64. Frid MG, Alasher AA, Dempsey EC, Stemath KR. Smoorh muscle cells isolated from discrete compartments of the mature vascular media exhibit unique planotypes and discinct growd capabilities.

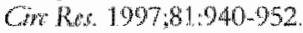

165. Frisbee JC, Stepp DW. Impaired NO-dependent dilation of skeletw muscle arterioles in bypertensive

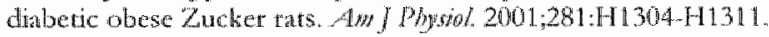

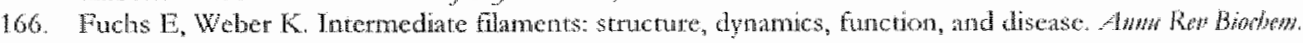
$1994,63 \cdot 345-382$

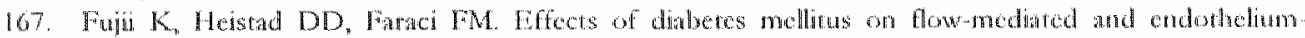
dependent dilatation of the lat basilar atrery. Shoke. $199223: 1494-1498$.

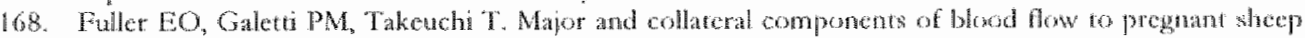

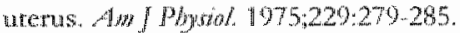

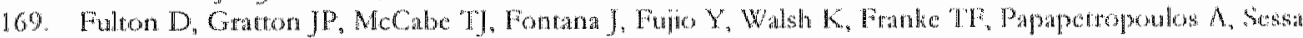

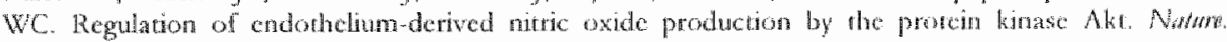
$1099,390,50 \%-601$.

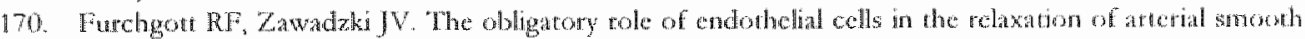
muscle by acryluoline. Natame. $1980288.373-376$

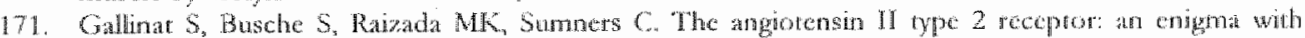

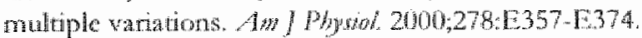

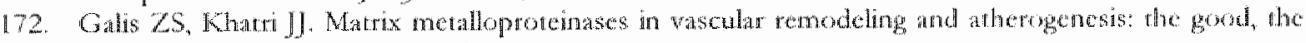
bad, and the ungly, Car Rer. 2002,90:251 262.

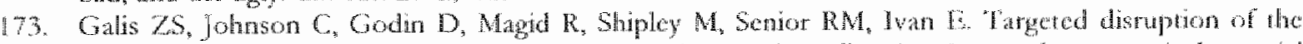
matrix metalloproteinase gene mopars smooth nuscle cell migraton and gecmerical ancral remodeling. Cir Res. 2002,91:852-859.

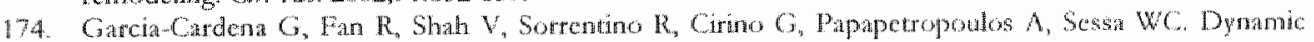
acrivation of endothelial nutric oxide synthase by 1 sp 90 . Nathe. 1998.302:821-1824.

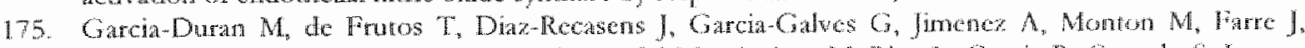

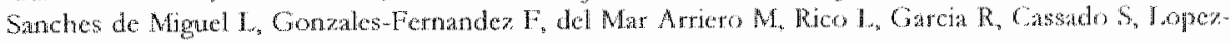




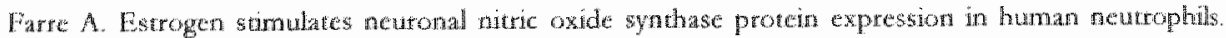

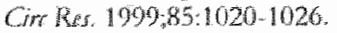

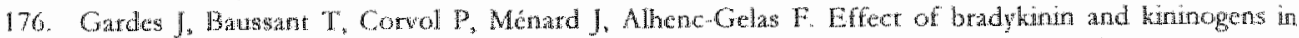

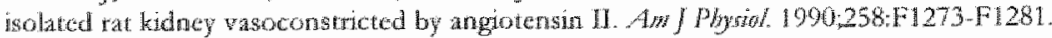

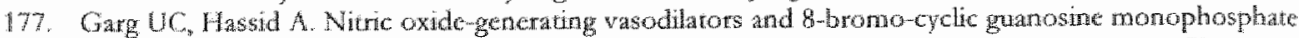

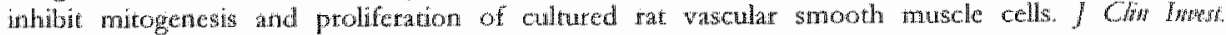
$1999,83: 1774-1777$

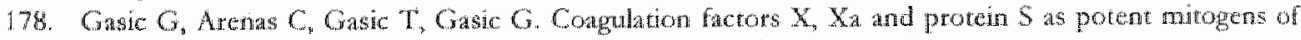

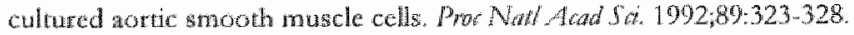

179. Gavras H, Brumer HR. Role of angiotersin and its inhbirion in hpertension, ischemic heat disease

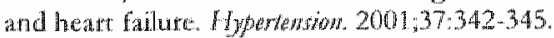

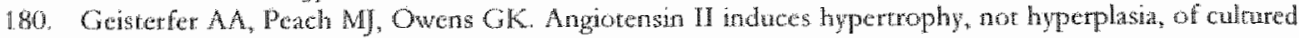

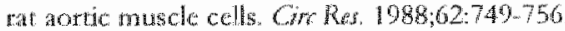

181. Gehard M, Roddy M. Creager S), Crager MA. Aging progresshely impairs endothelium-dependent

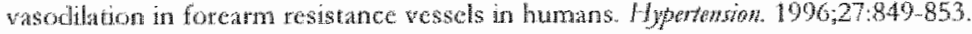

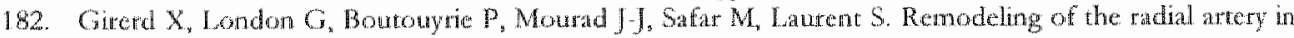

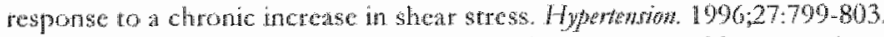

183. Giudiceli JF, Preslon JL, Glasson S, Richer C. Caproprit and hypertension development in the SHR. CW

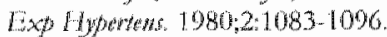

184. G]agov S. Intimal hyperplasia, wascular remodeling and the restenosis problem. Crawhron. 1994:80.28692891

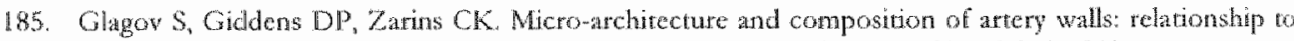

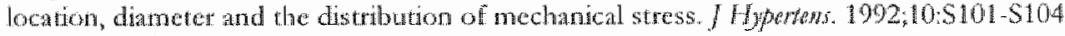

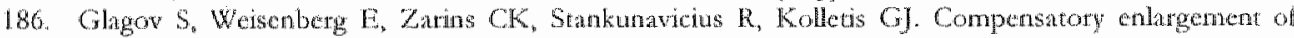

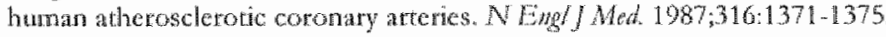

187. Godin D, Ivan E, Johnson C, Magd R, Gals 7s. Remodzling of catotid artery is associated with increased expression of matrix metalloptotomases in mouse blood flow cessacion moda. Canhation. $2000 ; 102 \div 2861-2866$

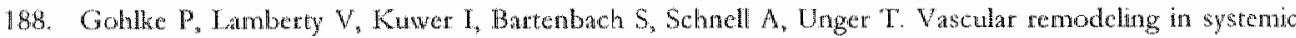
lyppertension. Ams Condiod. 199; $242 \mathrm{E}-7 \mathrm{~L}$

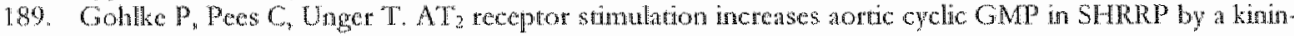
diependert mectuanism. Fypertentow. $1998,31.349-355$

190. Coldman $\mathbb{R}$ D, Chow YH, Dessew C, Dessev G, ErilissonJ, Goldman A, Khuon S, Kohnken R, Lown M. Miller $R_{n}$ Murphy $K$ Opal P, skalli $O$, Stratbe $K$. Dynamic aspects of cytoskeleal and karyoskaletal

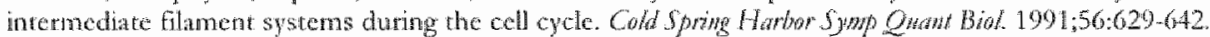

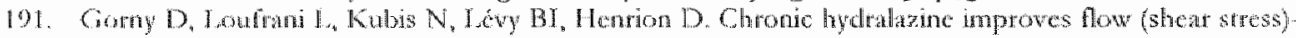

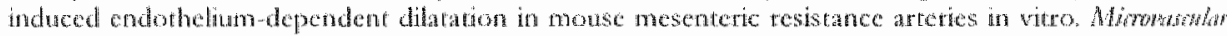
Reserwh $20111,64127-134$.

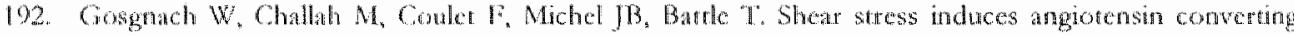

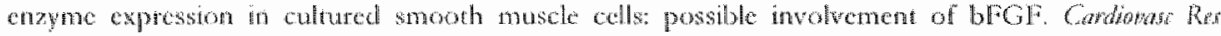
$2000,45: 486492$

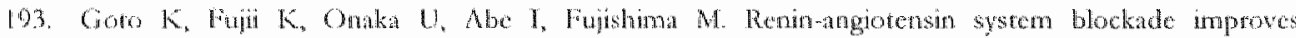

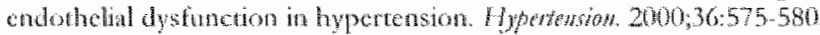

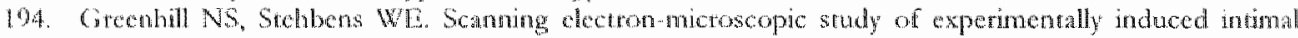

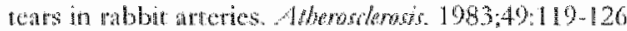

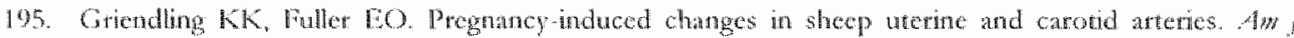
Pbyind $1985220: 11653-116605$

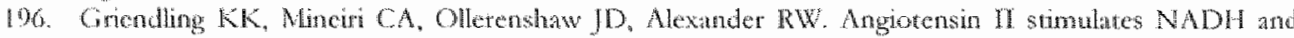

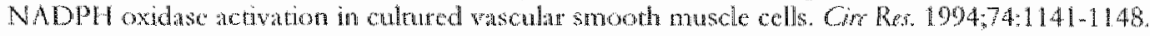

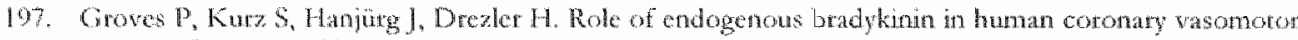
comerol. Cinthation. $1995,22,3424-3430$

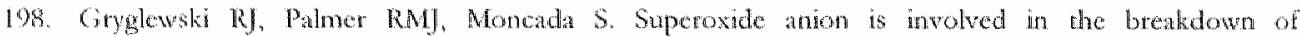

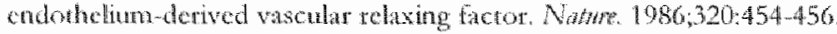

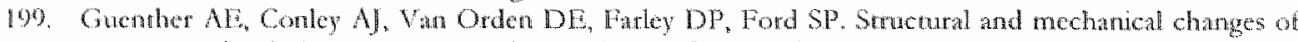

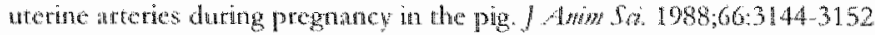


200. Guyton JR, Hartley $\mathrm{C}$. Flow restricion of one carond artery in puvenile tats inhibits growth of artenth diameter. Ans I Phoid $1985,248: \mathrm{H} 540-\mathrm{H} 546$.

201. Guzman $\mathbb{R}$, Abe Ka, Zarins $\mathrm{CK}$. Flow-induced arrerial entargement is inhibited by suppression of nutric oxide synthase in wivo. Surgty $1997 ; 122: 273-280$.

202. Guzman RJ, Krystkowiak A, Zarins CK Early and sustained medal cell scrivation affer aortacaval fistub creation in mice. J Sving Rer. 2002,108:112-121.

203. Haben RL. Anneser F, villinger A, Einhaupl KM. Angiotensin II induces endothelium-dependent

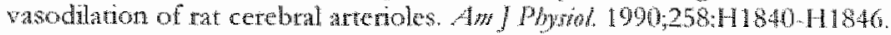

204. Hachamovitch R, Wicker P, Capasso JM, Anversa P. Alterations of coromary bood flow and teacre with aging in Fischer 344 rats. Axy I Plyniol. 1989;256:H66-H73.

205. Haefeli WE, Linder L, Luscher TF. Quinapriat induces arterial vasodilacion mediated by nirric oxide in humans. Hypertursion. 1997;30:912-917.

206. Hajdu MA, Heistad DD, Baunbach GL Effect of antilypertensive therapy on mechanics of cerebral arterioles in rats. Hypertansiow. 1991,17:308-316.

207. Hatpern W, Warshaw DM, Mulvany M. Mechancal and morphological propertics of anterial resistance vessels in young and old spontaneously hypertensive nats. Cin Rer. 1979,45:250-259

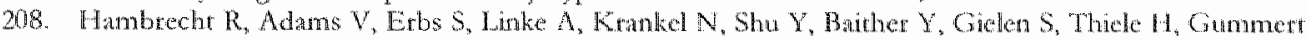
JfF, Mohr $\mathbb{F} W$, Schuler $G$. Regular physical activity improwes endothelial function in patients with coronary artery disease by increasing phosphorylation of endorhelial nitric oxide synthase. Cwowhion. 2003,25:3152-3158.

209. Hanemaijer R, Koow wjk $\mathbb{P}$. Le Cercq L, De Vree W], Van Hinsbergh VW. Regulation of matrix metaloproteinase expression in human vein and microvascular endohelial cells: effects of tumor necrosis factor $\alpha$, interleukin 1 and phorbol ester Biodunt ]. 1993;296:803-809.

210. Harmon KJ, Couper $\mathbb{L L}$, Lindiner $\mathrm{V}$. Strain dependent vascular remodeling phenotypes in indared mice. Am Pothol 2000; 156:1741-1748.

211. Hayashi K, Suzuki H, Sarua T, Segmental differences in angiorensin receptot subtypes in intertobulat

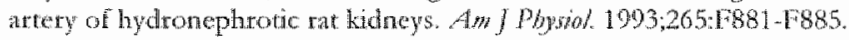

212. He $\mathrm{H}_{2}$ Venema V. GuX, Venema RC, Martero MB, Caldwell RB. Vasculat endothelial groweth factor signals endothelial cell production of nitric oxide and prostacycin through flk/KDR activation of s-Src. J Biol Chem. 1999;274:25130-25135.

213. Hedera P, Bujdakova P, Ttabner P, Pancak J. Stroke tisk tactors and development of collateral flow in carotid occlusive discease. Aita Newn/Srand 1998;98:182-186,

214. Hein I. Genetic deletion and overexpression of angiotensin 11 receptors. J Mo/ Med. 1998; 76756-763.

215. Helmke BP, Goldman $\mathbb{R D}$, Davies PF. Rapid displacement of wimentin intemedinte filancins in living endothelial cells exposed to flow, Cir Rer. 2000;86:745-752.

216. Hentiken EJ, Jacob S, Fogr DL, Dieze G]. Effect of chronic bradyinin admisnistation on insulin

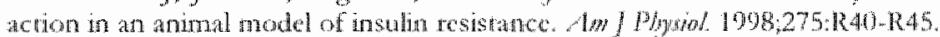

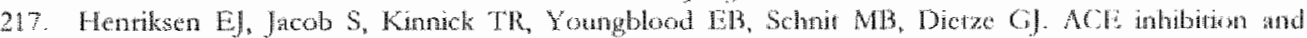
glucose transport in insulin-resistant musclos: Roles of bradykinin and nitric osids. Am I plygnt $1999,277 \cdot 12332-1336$.

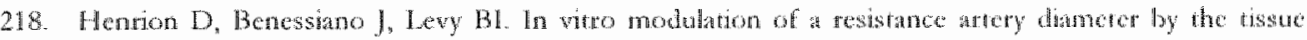
renin-angiotensin system of a large donor attery. Cire Res 1997; 80:189.95.

219. Henrion $\mathrm{D}$, Kubis $\mathrm{N}$, Lévy $B$ I. Physiological and pathophysiolegical functions of the $A T_{2}$ subtype

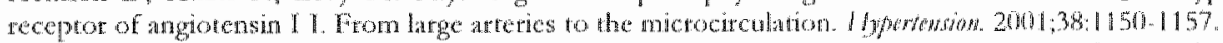

220. Henrion D, Terai F, Matrougui K, Duticz M, Boulanger CM, Colucci-Guyon L, Bablincr C, Briand P, Friedlander $G$. Poitevin P. Levy B1. Impaired flow indiced diation in mesenteric resistance arferies from mice lacking vimentin. / Clin lawes 1997;10029002914

221. Hermsteiner M, Zoltan DR, Doetsch J, Raschet W, Kuenzel W. Huntan chorionic gontadotropin dilates

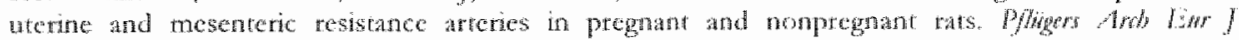
Ployrial.1999:439:186-194.

222 Heymann MA, Twamoto HS, Rudolph AM. Factors affecting changes in the neonatal systemic

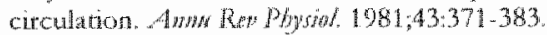

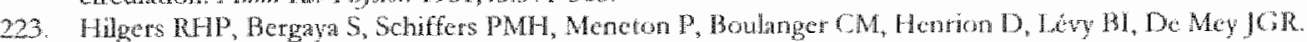
Uterine artery structural and functional changes during pregnancy in issue kalikfein-deficien mice. Arteriositer Thent Yase Biol. 2003; 23:1826-1832 


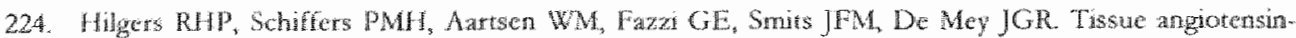

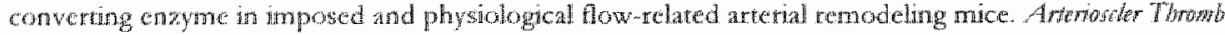
I. An Brol 2004; 24:In Press.

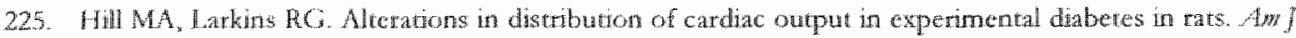
Pysal. $1989257: 11571$ H 1580 .

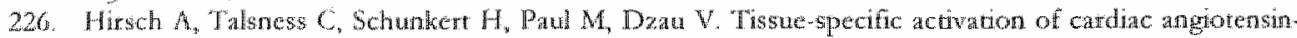

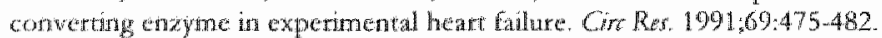

227. Hishikawa K, Nakaki T, Marumo $\mathbb{T}$, Hayash M, Suzuki H, Kato R, Satuta T. Pressue promores DNA

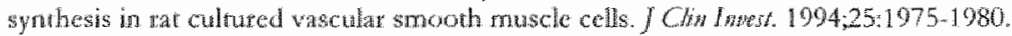

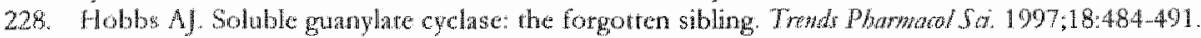

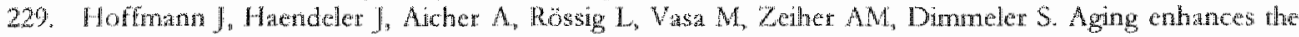
sensinviny of endothelal cels roward apoptotic stimuli Importane role of nitric oxide. Cir Re 2001.89709 .715

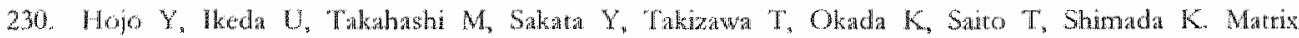
mothoprorinase-1 cxpression by interaction berween monocytes and vacular endothedisl cells. $J$ Mof Coll Cariol. $2000,32(8): 1459 w 1468$.

231. Holnower W, Kramsch DM, Famclant M, Madoff IM. Arteral wall metabolism in experimental

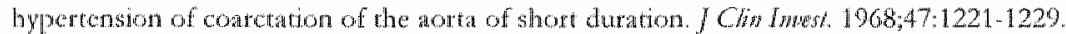

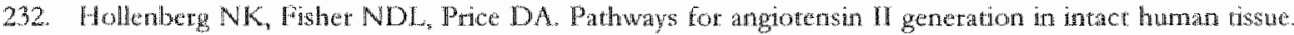

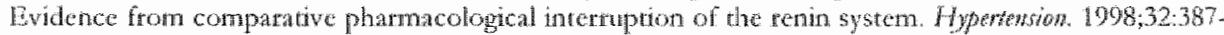
392

233. Holman R. Problems in the dynumics of blood flow. Y. Condition controlling collatetal circulation in the

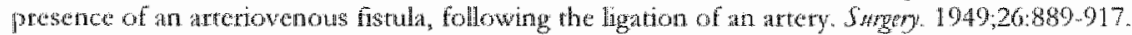

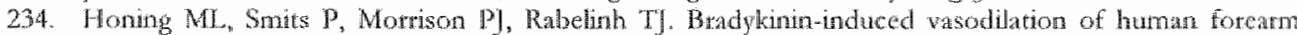

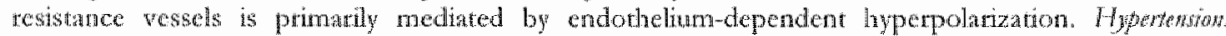
$2000,35 \cdot 1314-1318$

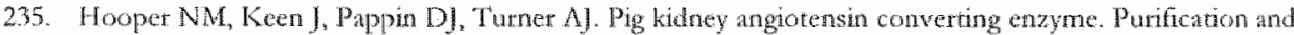
characterization of amphipathic and hydrophic foms of the enzyme establishes C-tominal anchomge

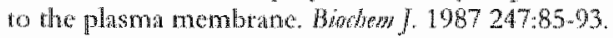

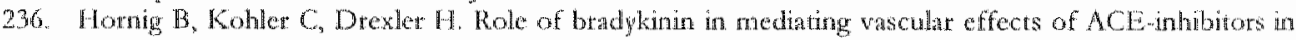

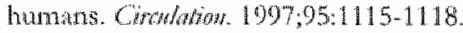

237. Hosomi N, Mizushige K, Othyana H, Rakahash T, Katai M. Hatanaka Y. Matsuo H, Kohno M Koriol JA. Angotensia converting enzyme inhibition widy enalaptil slows progressive intima-mediat thickening of the common carosid artery in patients wh non-insalin-dependent diabetes mollitus. Somke $2001,32: 15391545$

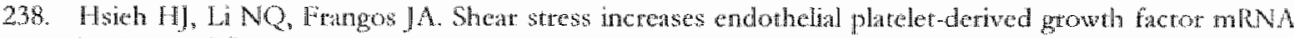

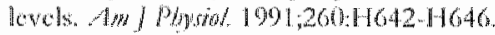

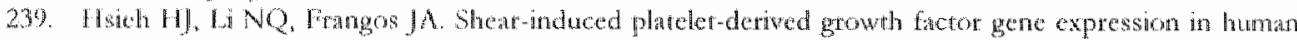

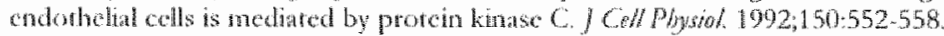

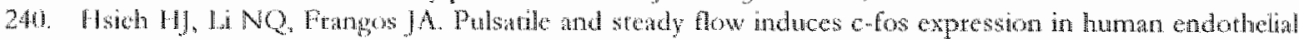
cols/ Cel/ponght 1993,154:143-151.

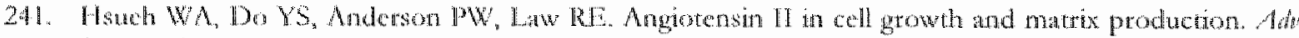

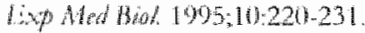

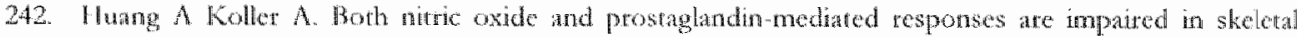

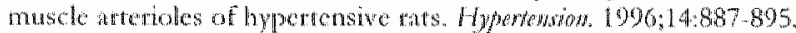

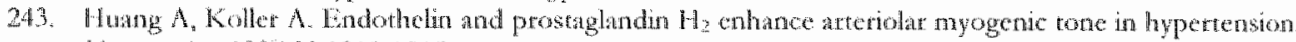
11) pertentom, $1907,30: 1210-1215$

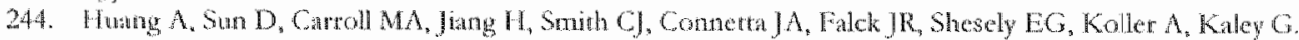

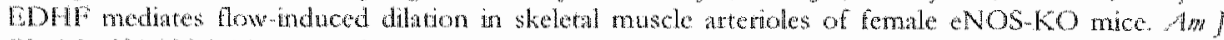
Wysiol 2001,280 :H2462-H2469.

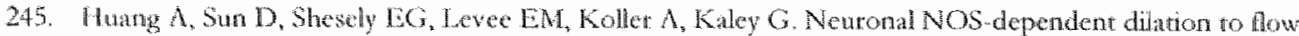

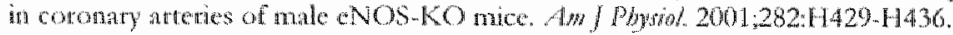

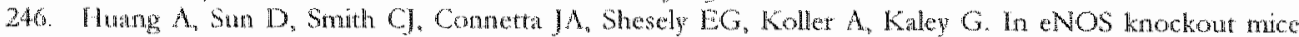

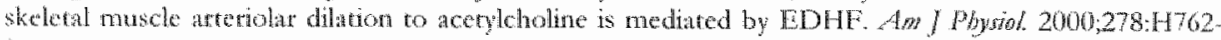
1708.

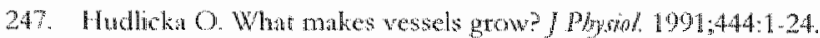


248. Hull SS Jr. Kadser L, Jaffe MD, Sparks V Jr. Endothelim-dependent fow-induced diturion of conine femoral and saphenous arteries. Blood wesceis, 1986;23:183-198.

249. Huonker M, Halle M, Keul J. Strucurat and functional adaptations of the cardiowateular sysem by training. Int/ Spors Med. 1996;17:S164-S172.

250. Hurcheson IR Griffth TM. Mechanotransduction through the endothehal cytaskeleton: mediaton of flow-but not agonst-induced EDEF release. Br J Phamod 1996:118720-726.

251. Futchinson HG, Hein L, Fujinaga M, Prat: RE. Modulation of vascular dewelopment and injury by

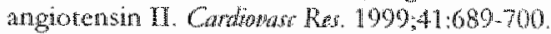

252. Ichiki 'T, Laboshy PA, Shiora C, Okuyama S. Imagawn $Y$, Fogo A, Nimum F, Lehikawa 1, Hogan BI. Inagami 'T. Effects on blood pressure and exploratory behavour of mice haking angerensin 11 rype-2 receptor. Natame $1995,377,748-750$.

253. Ichibara $S$, Senbonmatsu T, Ptice E, Ichiki T, Gafncy FA, Inagami T. Angibtensin II type 2 recoptot is essential for left ventricular hypertrophy and cardiac fibrosis in chronic angiotensin 11-induced Hypertension. Cinatutian. 2001;104:346-351.

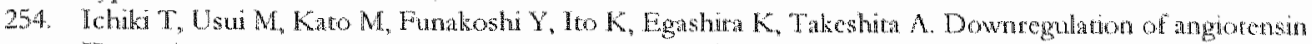
II type 1 receptor gene tratnscriprion by nitric oxide. Hymethion. 1998; 31:342-348.

255. Ingber DE. Integrins as mechanotransduction across the cell surface and through the cytaskelcton Sitente. 1991:38:841-848.

256. Ingber DE, Mechanochemical switching between growth and differentiation during fibroblact growth factor-stimulated angiogenesis in witro: role of cxertcelledar marix. / Col Biol 1991:109:317-330.

257. Irace $C$, Gnasso $A$, Cirillo F, Leonardo $G$, Ciamei $M$, Crivato $A$, Renzulli $A$, Cotrufo $M$. Arterial remodeling of the common catotid atery after aortic valve rephacencon in paticurs with aortic stenosis. Stroke $2002 ; 33: 2446-2250$.

258. Ishida S, Hanasaki S, Kannekou M, Yoshitama T, Nakana F, Yoshikawa A, Kataoka T", Saihara K, Minagoe $S$, Tei C. Advancing age is associated with diminished wascular remodeling and impaired pasodilation in resistance coronary arteries. Coron Aryty Dis. 2003; 14:443-449.

259. Tto WD. Aras M, Scholz D, Winkler B, Houn P, Schraper W. Angiogenesis bue not collateral growth is associated with ischenia after femoral artery ligation. Am J Ptystot. 1997;273:H1255-11265.

260. Twan E, Khatri JJ, Johnson C, Magid R, Godin D, Nandi $S$, Lessner $s_{8}$ Galis ZS. Expansive arterial remodeling is associated with incrensed neointimal macrophage fostn cell content. Cituthation. $2002 ; 105: 26866-2693$.

261. Iwasaki $H$, Eguchi $S$, Marumo F, Hirara $Y$. Endohelin-1 sumulates DNA syothesis of vaseular snooth-

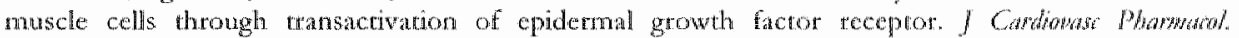
$1998 ; 31: \$ 182, \$ 184$

262. Jackson WT. Arteriolar tone is determined by activity of ATP-sensirive ponssim channols. Am I Ptysiol. $1993 ; 265: H 1797-\mathrm{H} 1803$.

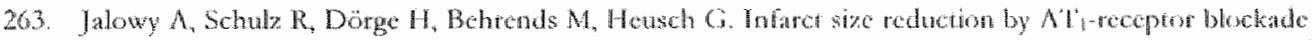

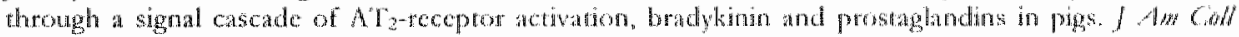
Cardicil 1998:32:1787-1796.

264. Jamal $A$, Bendeck M, Jangille BL. Structural changes and recowery of function after arieral ingury. Alteriaster Throwt 1992;12:307.317.

265. Janiak $P$, Libert $O$, Vilane JP. Role of the renin-angionensin systen in noontima fommation after injury in rablins. Hypentasions. 1994:24:671-678.

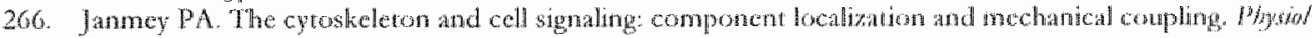
Res. 1998;78:763-781.

267. Jawien $A$, Bowen-Pope DF, Lindner $Y$, Schward SM, Clowes AW. Platdet-derived growth factor promotes smooth muscle migration and ineimal thickening in a rat model as balloran angioplasty. $/ \mathrm{Cl}$. Ihw ar 1992,89:507-511.

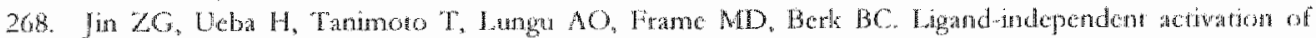
vascular endothelial growth factor receptor 2 by flud shear stress regulates actiwaticofic of endorhelial nitric oxide synthase. Cir Rer 2003,93-354-363.

269. Jomnides $\mathbb{R}$, Haefel WE, Linder $L$. Ruchat $V$, Bakkali EH, Thuillez $C$, Luscher TT. Netrie oxide is

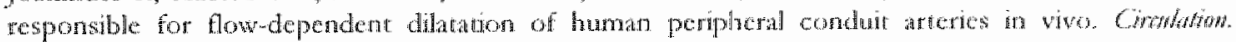
1995:91:1314-1319.

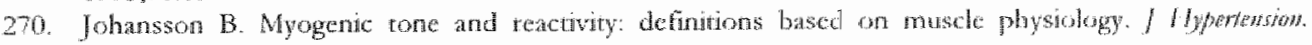
$1089 ; 7.55-58$. 


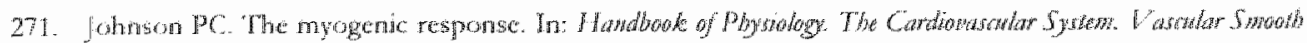

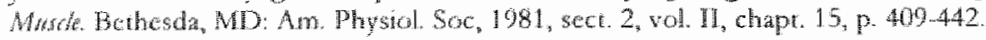

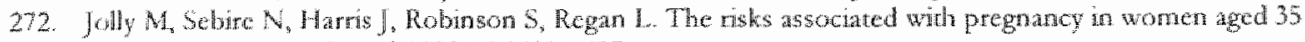

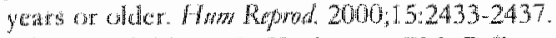

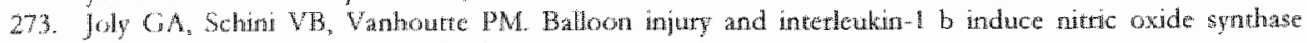

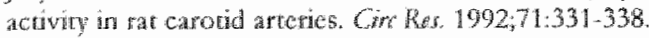

27a. Jones $G \mathrm{~T}$. Martin BJ, Stchbens WE. Endothelim and etastic reats in whe afferent arteries of

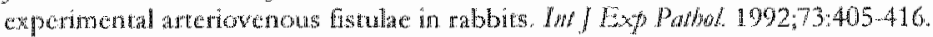

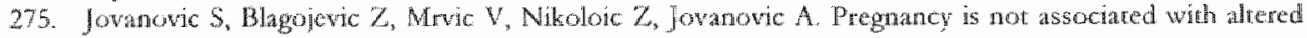
momplogy of the fomoral artery. Hwh Reprod 1999;14:1885 1889.

276. Jovarowic S. Jowanowic A. Remodeling of guneapig aorta durng pregnancy a selective alterations of

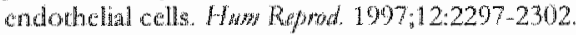

277. fowanowic S, Jowanovic A. Pinegamey is associated with hyporophy of camotid arrery endothelial and smooth muscle cells. Hom Reprod 1998;13:1074-1078.

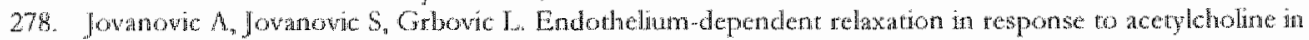
pregnant guincapig therine artery. Hom Reprod. 1997,12:1805-1809.

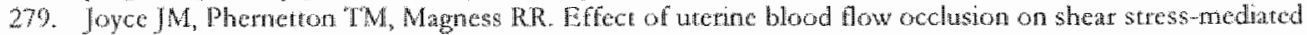
nitric oxide production and endothelial mitric oxide synthace cxpression during ovine pregnancy. Binl Repw 2002,$67 ; 320320$

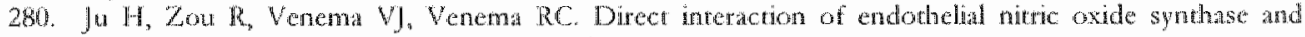

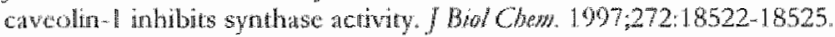

281. Kamata Ka, Migata N, Abiru T, Kastya $Y$. Functional changes in vascular smooth muscle and

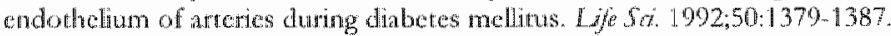

282. Kamina $M$, Togawa T. Adaptive regulation of wall shear stros to flow change in the canine catotial

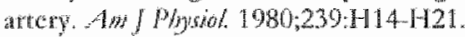

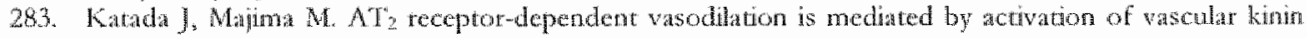

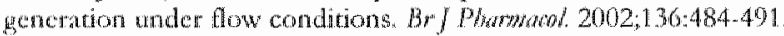

284. Katoh Y, Perasamy M. Growth and ditefentiation of smooth mosele cells during wasculat dewelopment. Thends Cart Mod 1096,6:100-106.

285. Keidar 5 , Attas J. Coleman $R$, Wrth $k$, Scholkens $B$, Hayck $T$. Atenuation of atheroscterosis in apolpoprotein Edeficient mica by rampril is disociared from its anthypertensive ctect and from

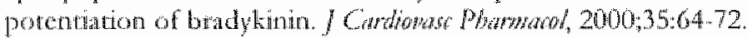

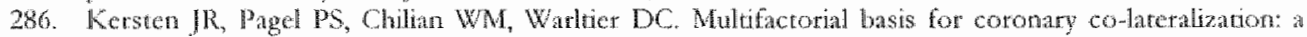
complex adaptive response to ischenum. Comblow Res 1999;43:44-57.

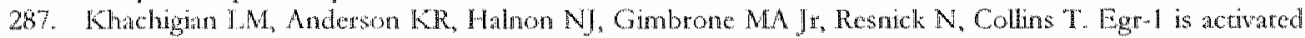

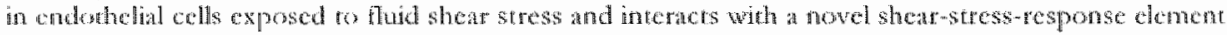

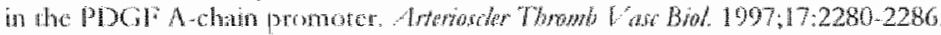

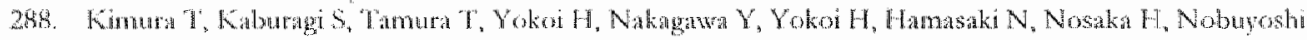

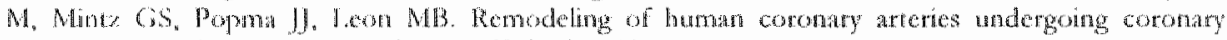

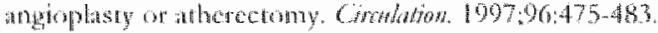

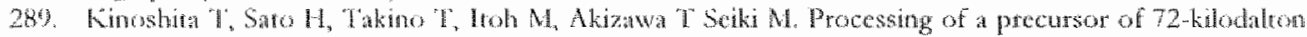

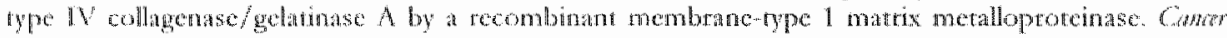
Ra. 1906 56:2535-2538.

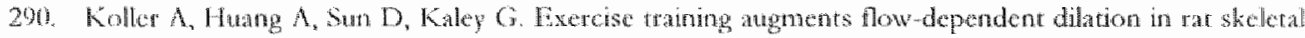
nuscle anterioles. Con Res 1995;70:544-550.

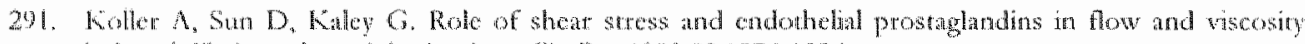
finduced dithion of atceloles in vitro. Cir Rer. 1993,72.1276-1284

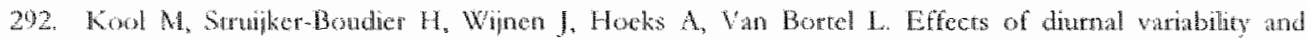

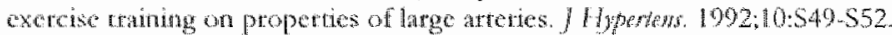

203. Kershunow $V A$, Berk BC. Flow indaced wascular remodeling in the mouse a model for carotid intima-

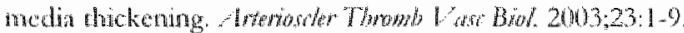

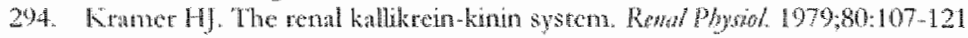

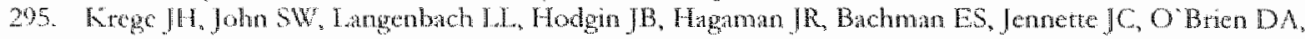
Smithes O. Makentemalc differences in fertily and blood pressure in ACE-deficient mice. Nantre $1995 ; 375: 146-148$ 
296. Kubis N, Checoury A, Tedgui A, Lévy BI. Adaptive common courodid arteries remodeling afrer uniluteral internal carotid artery occlusion in adult patients. Conglionat Res, 2001:50:597-602.

297. Kuchan M], Frangos JA. Shear stress negulates endothelin-1 release wa protein kinase C and cGMP in cultured endothelial cells. Ams P bysiol. 1993; 264:14150-4156.

298. Kuchan MI. Frangos JA. Role of calcium and calmodultin in flow-induced ritric oxide production in

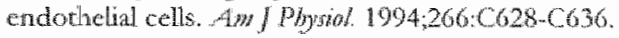

299. Kumar A, Hoover JI., Simmons $C A$, Lindner $V$, Shebuski $B]$. Renodeling and neointinal formation in whe carotid artery of normal and $\mathrm{P}$-selectin-deficient mice. Ciftatwion. 1997,96:4333-4342.

300. Kumar $A$, Lindner $V$. Remodeling with neointimat formation in the mouse carotid artery after cessation of blood flow. Anterionder Thromb lat Biol 1997;17:2238-2244.

301. Küng CF, Luscher TF. Differene mechanisms of endothelial funcrion with aging athd hypertension in at aorta. Hypertension. 1995:25:194-200.

392. Kuo L, Davis MJ, Chilian WM. Endohelim dependent, flow-induced dilation of isolated cononary arterioles. Ans J Pigrod 1900259:H1063-H1070.

303. Kuzuya M, Kanda S, Sasaki T, Tamaya-Mori N, Cheng XW, Itoh "l, Irohara $S$, Iguchi $A$. Deficiency of gelacinase A suppresses smooth muscle cell inwasion and development of experimental intimal hyperplasia. Cimwlations. 2003;108:1375-1381.

304. Lacolley P, Challande $P$, Boumaza $S$, Cotruet $G$, Laurent $S$, Boutouyrie $P$. Grimand IA, Patulin D, Lamaziere JM, Li Z. Mechanical properties and structure of carotid areries in mice lacking desmin.

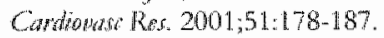

305. Lakatta EG. Arterial and cardiac aging major shareholders in cardiovascular disease enterprises. Part IVI: cellular and molecular clues to hatr and arterial aging. Cowwdano 2003;107:490-497.

306. Dan Q, Mercurius KO, Davies PF. Stmulation of transeription factors NE happa $B$ and AD-1 in endothelial cells subjected to shear stress. Biobem Bioplyys Res Common. 1994:201:950-956.

307. Lang MG, Noll G, Eüscher TF. Effect of aging and hypertension on contractility of resistance artevies: modulation by endothelial factors. Amy Physol. 1905:269:-H837-H844.

308. Langille BL. Hemodynamic factors and vascular wall disease. In Silver $\mathrm{E}_{2}$ ed. Condionuistor pathology. 1991; New Yok, NY: Churchill Livingstone: 131-154.

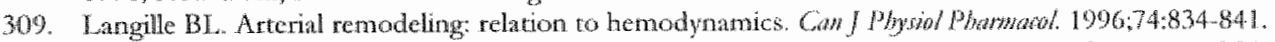

310. Langille BI, Bendeck MP, Keeley FW. Adaptations of carotid anteries of young and matute vabbits to reduced carotid blood flow. Am I Phyrod. 1989;250\%1931-H939.

311. Langille BL, Brownlee RD, Adamson SL. Perinatal growth in lambs: relation to blood flow changes at birth. Aly I Physiol. 1900;259:H1247-H1253.

312. Langille BL, ODonnell $\mathrm{F}$. Reductions in atterial diameter produced by chronic decreases in blood flow are endothelial-dependent. Soieme. 1986;231:405-407.

313. Larsen E, Celi A, Gilbert GE, Furie BC, Mrban JK, Bonfunt R, Wagner DD, Furie P, PADGMM protein: a receptor that mediacs the interaction of activated plateles with neutrophils athd monocytes. Cell 1989:59:305-312

314. Lash JM, Bohlen HG. Time- and orderdependent changes in functional and NO-sncdiated dillaton during exeroise training. J Appt Plysiol 1997:82:460-468.

315. Cauth M, Berger MM, Caitaruza M, Hocker M. Elevated perfusion pressute uprcgulates endothelin-1 and endothelin $B$ receptor expression in the abbit carotid artery. Hypetwhion. 2000; 35:648-654.

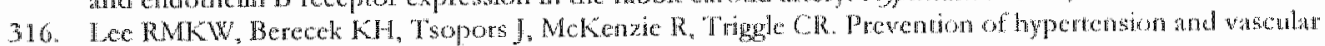
changes by captopril reatment. Hypertowsion. 1991:17:141-150.

317. Lehman DK, Chism J. Pregnancy onteome in medically complicated atid uncomplicanted patients aged 40 years on older. Am J Obste Gytion. 1987;157:738-742.

318. Lehoux 5 , Tedgui A. Signal transduction of mechanical stresses in the vascular wall. Mypertenton,

1968:32-338-345.
319. Levy B1, Michel JB, Salzmann JL, Poiresin P. Devissaguet M, Scabert ME, Safar MT. Long-tem effects of angiotensin-convercing enzme intubition on the arteral wall of adult spontancously hypenensive rars. 4 I I Catrithol. 1993;71:8e-16e.

320. Li J S, Turgeon A, Schiffin EL. Effect of chronic treatment with wo different ETA sclective cndothelin receptor antagonists on blood pressure and small artery structute of deoxycorticosterone anceratc (OOCA)-salt hypettensive rats. Am J bypertens. 1998;11:554-562. 


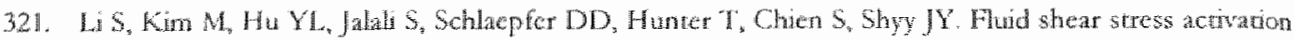

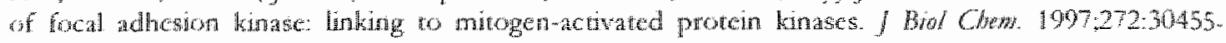
30462 .

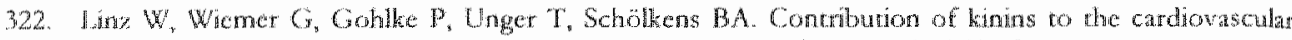

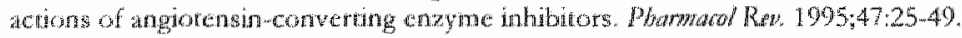

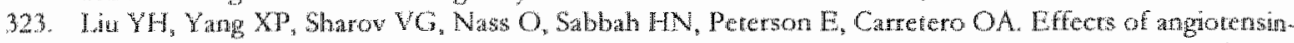

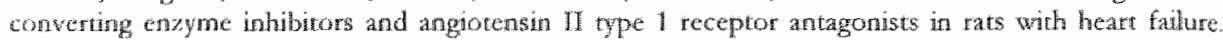

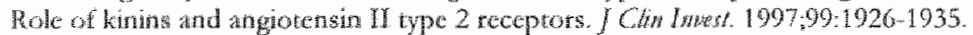

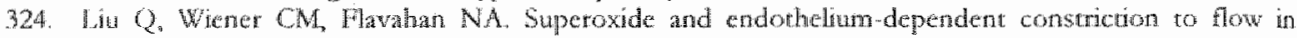
porcine small pulmonary arteries. Br J Pharmatal. 1998:124:331.336.

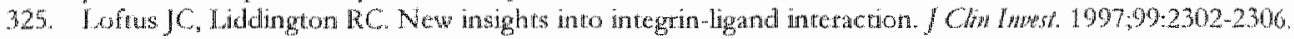

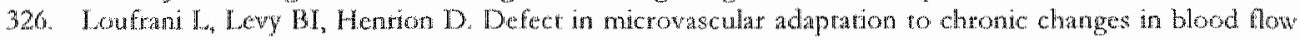
lakking the gene encoding for dystrophin. Cire Rer 2002;91:1183-1189.

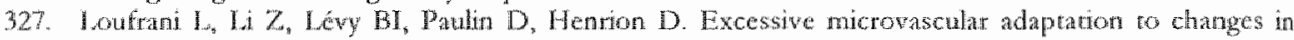

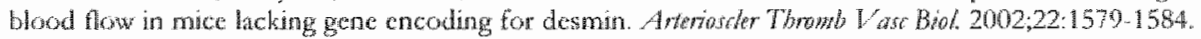

328. Loutran L, Matrougui K, Gorny D, Duricz M, Blanc I, Levy BI, Henrion D. Wow (shear stress)-induced endothelum-dependent diation is altered in mice lacking the genencoding for dystrophin. Cinotwon. $2001,103.864-870$.

329. Lu X, the JB, Wang GR, Gregersen M, Kassab GS. Remodeling of the zero-stress state of femort

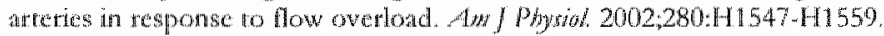

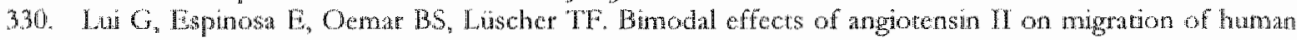
and rat smouth muscle cells. Direct stmulation and indirect inhbition wiat ranstorming growth facror

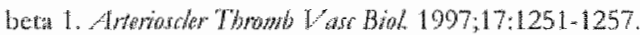

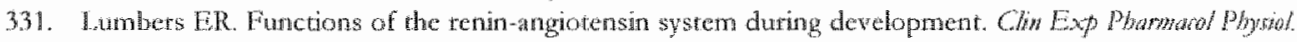
$1995 ; 22,499.505$

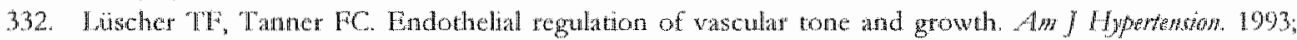
$6.2835-2939$

333. Hon RT, Runyon-Mass A, Davis HR, Glagon S, Zarins CK. Protecton from atherosclerotic lesion Formation by reduction of artery wall motion. I How $\$$ mog. $1987,5,59,67$.

334. Madeddu P. Papaglit PP, Demoncis MP, Varoni MV, Mataccio MC, Anana V, Glorioso N. Earty

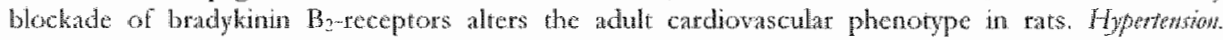
$1095,25: 453+459$

35. Magness RR, Phometon TM, Zlyeng J. Systemic and uterine blood flow distribution during prolonged

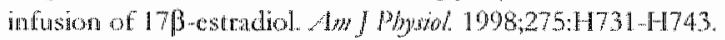

336. Magness RR, Rosenfeld $C R$. Hassan $A$, Shat PW. Endohelial vasodilator producton by uterine and

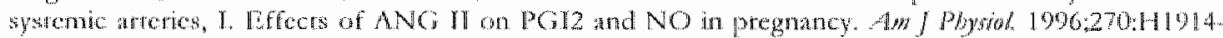
$1102 \%$

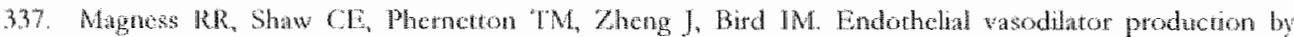

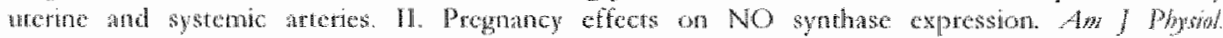
$1907272: 111.73040$

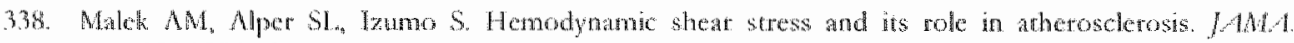
$1909.2822035+2042$

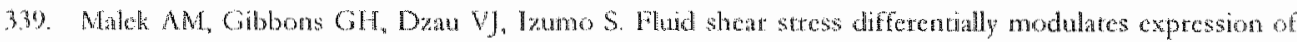

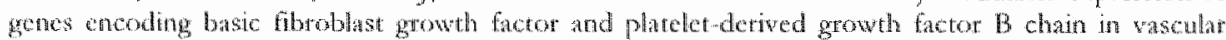

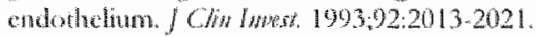

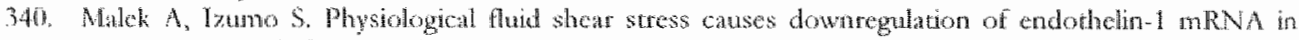

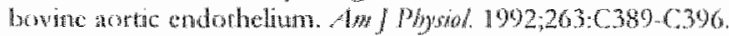

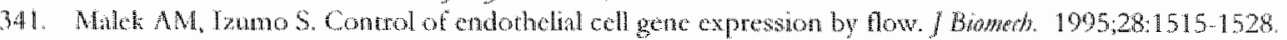

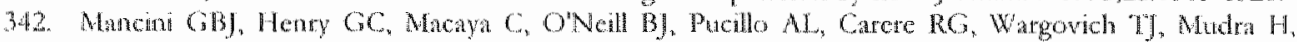

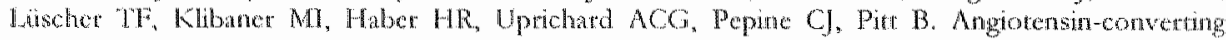
cnayne inhibion with gunapril inproves endohelial vasomotor dysfuncton artery disease. The

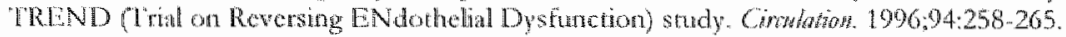

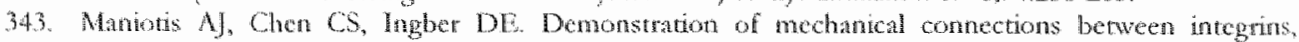

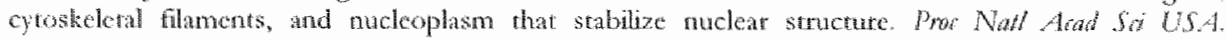
$1907,94: 348-854$ 
344. Marcedu $F$, Lussier A, Regoli D, Giroud J. Pharmacology of the kinins their refewatec to tissue ingury and inflammation. Gen phasmarol. 198314:209.229.

345. Marceau F, Sabourin $T$, Houle $S$, Fottin JP, Petatclerc $\mathbb{E}$, Molinaro $G$, Adam A. Kinin receptors:

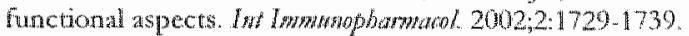

346. Margolius HS. Kallikreins and kinins. Some unanswered quesrons about systen characteristics and roles in human discase. Hypertmion, $1995 ; 26: 221-229$.

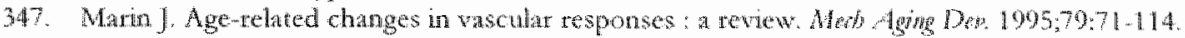

348. Masuda H, Kawamura K, Tohda K, Shozawa T. Sageshima M, Kamigas A. Increase in cndothelial cell

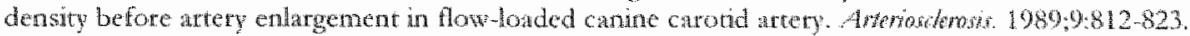

349. Masuda $H$, Sugita $A$, Zhuang $X$ J Pathology of the arteries in the central nervous system with special reference to theje dilatation to blood flow. Nestopathology 2000;20:98-103.

350. Masuda H, Zhwatrg V], Singh TM, Kawamuma K, Murakami M, Zarins CK, Glagon S. Adaptiwe remodeling of internal elastic lamina and cndothelial lining during flow-induced aterial enlargenent. A werionder Thromil Van Brol. 1990;19:2298-2307.

351. Mamoba T, Shimoliawa H, Kubota H, Morikawa K, Fujiki T, Kunihiro I, Mhakay Y, Hirakawa $Y$, Takeshita A. Hydrogen peroxide is an endothelum-derived hyperpolarizing factor in humin mesenterio anteries. Brodrens Bioplys Rer Common. 2002;209:909-913.

352. Matrougui K, Eskidsen-Heimond YE, Hebeter A, Henrion D, Levy BI, Tedgui A, Mulvany MJ. Angotensin II stimulates extracellular signal-regulated kinase acrivity in intact pressutized at mesenteric resistance atteries. Hopertenstom. 2000; 36:617-1621

353. Marrougui $\mathrm{K}$, Loufran $\mathrm{L}$, Heymes $\mathrm{C}$, Levy BI, Hention D. Activation of $\mathrm{AT}_{2}$ receptors by endogenous angiotensin II is involved in tlow-induced dilation in tat tesistance arteries. Hypertewion. 1999; 34:659-665.

354. Matsumoto $T_{*}$ Kanamaru $\mathbb{K}$, Sugigama $Y$, Mutata $Y$. Endodhelium derived relasation of the pregnant and nonpregnant canine utcine atrery. I Repord Mad. 1992,37,529-533.

355. Matz RL, de Sotomayor MA, Schote C, Stodter JC, Andranesitohaina R. Vasculat bed herescogeneity in

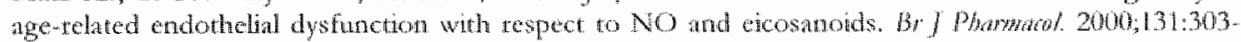
311.

356. Medhora M, Bousamn M, Zhu D, Somberg L, Jacobs ER. Upregularion of collagens detected by gene

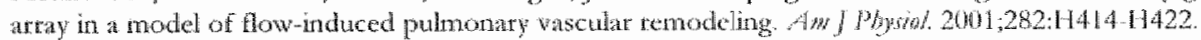

357. Meininger GA, Davis, MI. Cellular mechanisms involved in the vascular myogenic response. Ans. J. Plyyinol 1992;263:H647-H659.

358. Mello $G$, Parretti $E$, Gensini F. Stichi $E$, Mecace $F$, Scarselli $G$, Genuardi M, Abbate R, Fatini $C$ Maternal-fetal flow, negative events, and preeclampsia. Hypertensiom. 2003;41:932-937.

359. Meneton P, Bloch-Faure M, Hagege AA, Ruetten H, Huang W, Bergaya S, Ceiler D, Gebling D, Martins I, Salmon $G$, Boulanger CM, Nussberger $N$, Crozatier $B_{3}$ Gasc ] M, Heudes D, Binaneval P, Doetschman "T, Menard J Alheac-Gelas E. Cardiovascular abnomalines with momal blood pressure in tissuc

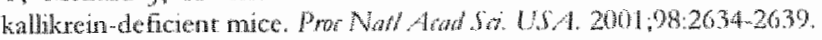

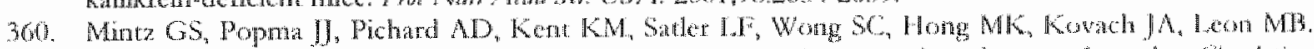

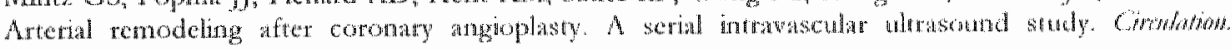
$1906,94: 35-43$.

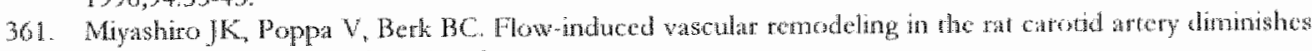
wirh age. Cim Res. 1997,81:311-319.

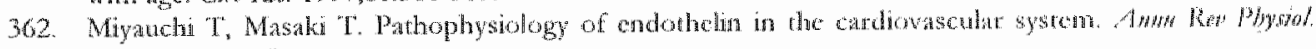
1999,61:391-415.

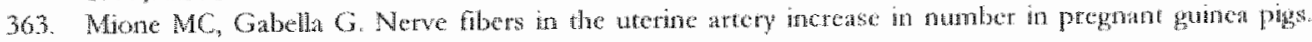
Nesmomort. $1991,24537.540$

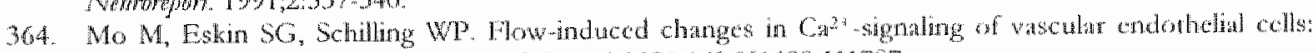
effeces of shear stress and ATP. Am f Pyygrat 1991;260H1698-111707.

365. Mombouli JW, Vanhoutte PM. Kintus and endothelum-dependene relaxations bo converting enzyme

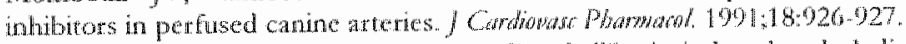

366. Mombouli JV, Varhoutte PM. Kinins mediate kallikrein-induced endothelium-dependene relaxations in

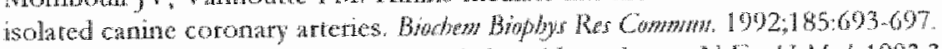

367 . Moncada S, Higgs A. The L-arginine niwic oxide pathway. N LnglJ Mat, 1993,3202002-2012

368. Mondy JS, Lindner $V$, Myashiro IK. Berk BC, Dean RH, Genry RL Platelc-derived grewh Gachor

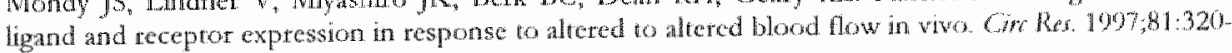
327. 


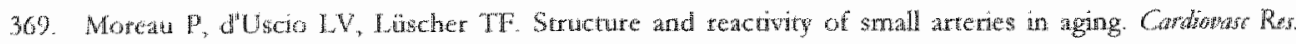
$1998,37247.253$

370. Mor E, Komor K, Yarnoka T, Tani M, Kataok C, Takeshita A, Usu M, Egashina Kugimach $\mathrm{K}$. Essential role of monocyte chemoatuctant proten-t in dewelopment of restenotic changes (neointumal hyperplagia and consurictive remodeling after balloon angoplasty in hpercholesterolemic rabbits. Ginhtwon. 2002 : $1052005-2910$

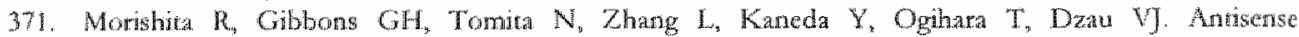

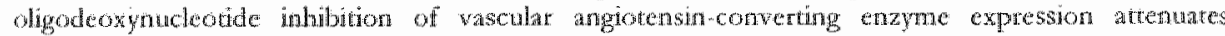

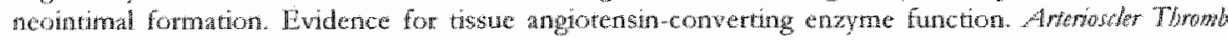
Task $1301,2000,20,915,922$

372. Morishim T, Tsutsui M, Shimokawa H, Hotuchi M, Tanimoto A, Sudia O, Tasalki A, Hung PL, Sasagur:

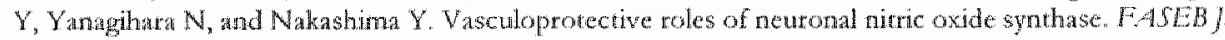
$2002 ; 16: 1904-1996$

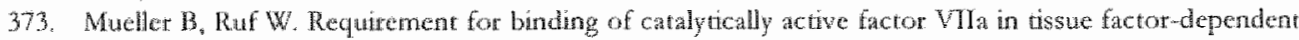

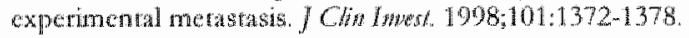

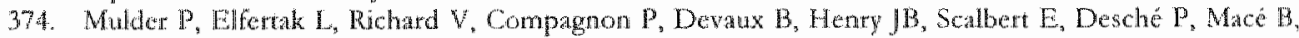
Thuilez C. Perpheral artery structure and andotyelial functon in heart falure effec of $A C E$ inhibion. Am / Plysial $1906,271 \mathrm{H} 460 \mathrm{H}-\mathrm{H} 47$

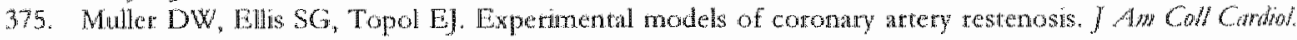
$1992 ; 19: 416432$

376. Muller. Delp JW, spicr SA, Ramseg MW, Delp MD Aging impats endothelium-dependent vasodilaton

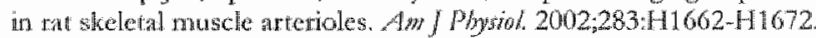

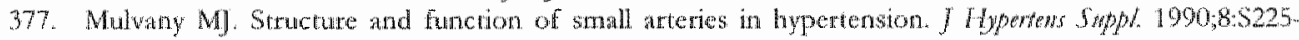
5232.

338. Mulvan M. Small artery remodeling and significance in the dewelopment of hypertension. Nen: Pbysol Sa. $2002,17: 105 m 109$

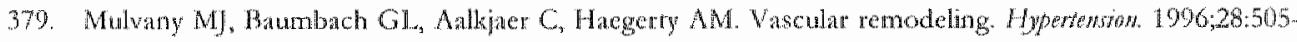
506.

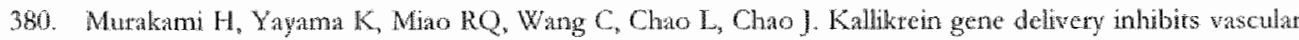
smooth muscle cell growth and neomtima formation in the rat atery after balloon angioplasty Hopertostion. $1999,34: 164-170$.

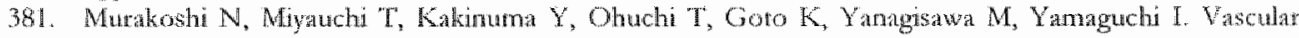

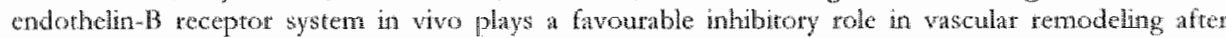

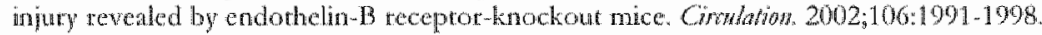

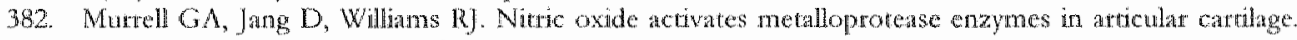

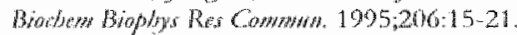

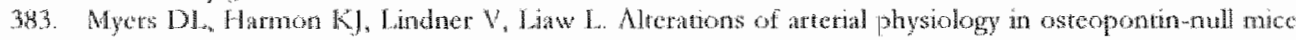

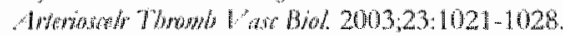

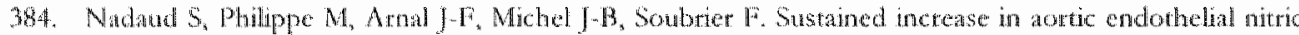
oxide synthase expression in vivo in a model of chronic thigh thw. Cor Rns $1996779857-863$.

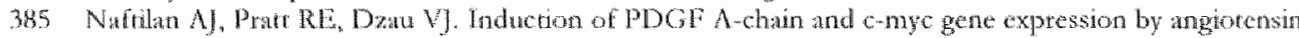

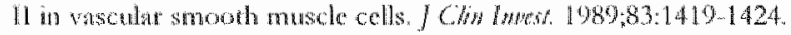

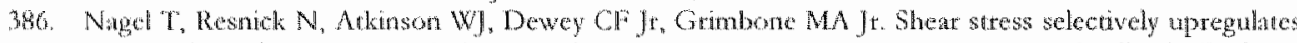

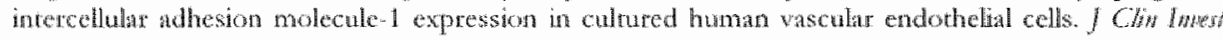
$1994,04895-891$

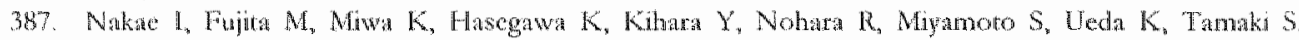
Sastyma S. Age deperadent impanment of cotonary collateral development in humans. Heant Teosh $2000,15,176-180$

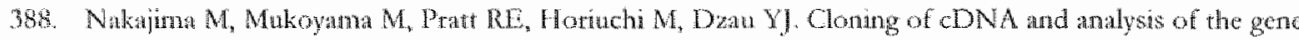

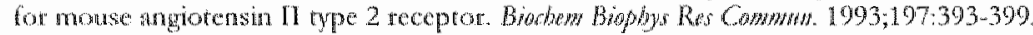

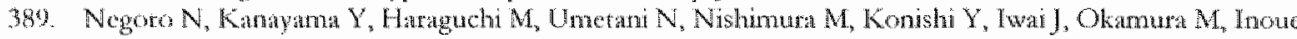
T, Takod T. Bhod pressure regulates platelet-derived growth Getor A whan gene expression in wascula

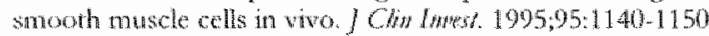

300. Nekon SH. Sreinshnd OS, Suresh MS, Lee NM. Pregnancy angments nitrie oxide-dependent dilator responses to acetycholine in the human uterine artery. How whw 1998;13:1361-1367. 


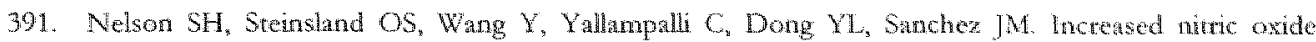
synthase activity and expression in the human uterine artery during ptegrancy. $\mathrm{CH}$ Ker. 2000,87.406-11.

392. Ni X, May V. Bras K, Osol G. Pregnancy auganene urerophcental wacular endotholial growh factor gene expression and wasodilator effects. Am J P/ysio/. 1997,273:1938 H944.

393. Ni Y, Meyer M, Osol G. Gestation increases nitric oxide-medated vasoditurion in met uterine arteries

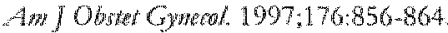

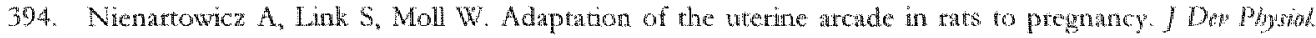
$1989: 12: 101-108$

395. Nishida K, Harrion DG, Navas JP, Fisher AA, Dockery SP, Uematsu M, Nerem RM, Alexandet RW, Murphy T]. Molecular cloning and chancterization of the constitutive bowite aortic endothelal cell

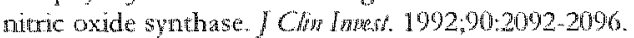

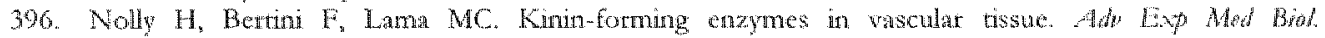
11983;156:3904407.

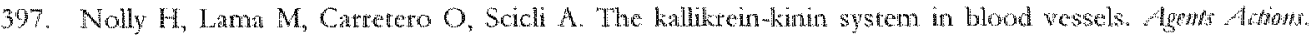
$1992: 38: 1-9$

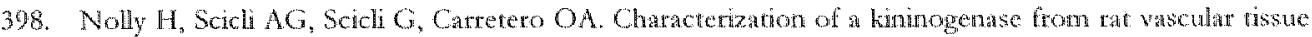

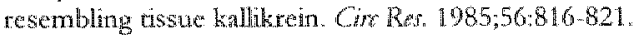

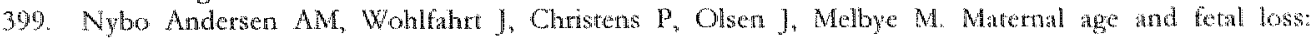
population based register linkage study. BM, $2000 ; 3201708-1712$.

400. Ohro M, Cooke JP. Dzau V], Gibbons GH. Fluid shear stress induces endothethat transtoming gronth

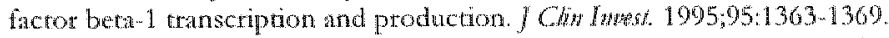

401. Okada K, Bernstein ML, Zhang W, Schuster DP, Botrey MD Angotentin-converting enzyme

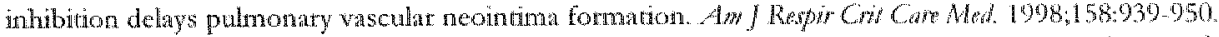

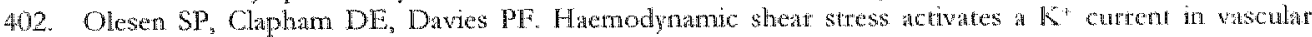
endothelial cells. Natur. 1988;31:168-170.

403. Oniki $\mathrm{T}$. Iwakami $\mathrm{M}$. Is aterial remodeling truly a compensitory biological rencton A mechanical de formation hypothesis. Aherasterosio. 1997;132:115-118.

404. Osol $G$. Myogenic properties of blood vessels in vitro. Wi The Resistatec Vascuature. 1991: JA. Wexan, W. Halpern, and M.J. Mulwany, editots. Humma Press. Totowa, NJ. 143-157.

405. Osol G. Mechanotransduction by wascular smooth muscle. / Var Rer $1995,32: 275-292$.

406. Osol G, Cipolla M. Pregnancy-induced changes in the three-dimensional mechanical propertics of pressurized gat uteroplacental (radial) arteries. Am J Obsh Gynol. 1993, 168:268-74.

407. Osol $G$, Cipolla $M$. Interaction of myogenio and adrenergic mechanisms in isolated, pressurized uterine radial atteries from late pregnant and nonpregnant rats. Am f Pbyol. 1903, 168:607-705.

408. Osol $G$, Cipolla $M$ Hypertophic and hyperplastic effects of pregnathey on the rat urerine arrenal wall.

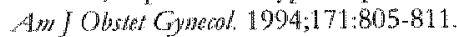

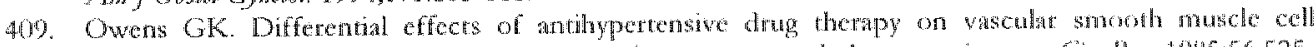

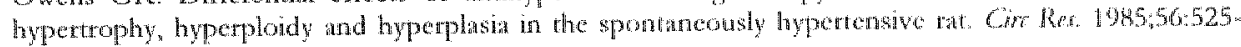
536

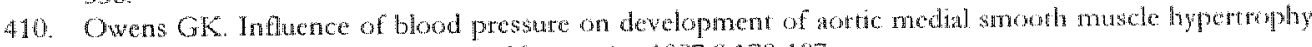
in spontaneously hypertensiwe rats. Hypowengh. 1987,9:178 187.

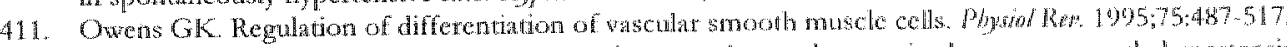

412. Owens GK, Schwart 5 . Aleratons in vasular smogth muscle mass in the spontaneonstypentensive

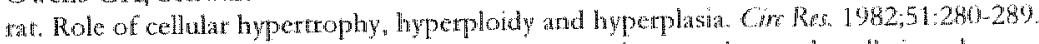

413. Oza N. Schwarz J, Gould $\mathrm{H}$, Levinsky $\mathbb{N}$. Rat aotic smooth muscle cells in culture cxpress kallikein

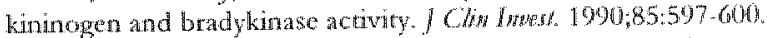

414. Palmer RM, Ferrige AC, Moncsda $\$$. Nitric oxide release accounts for the biological activity of

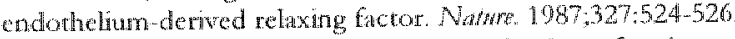

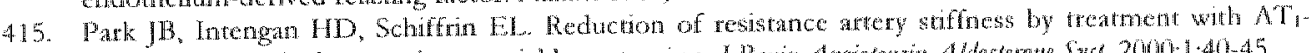

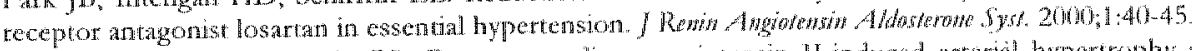

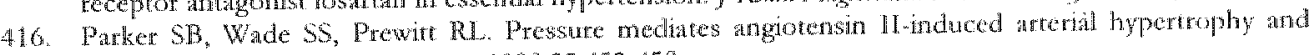
PDGF-A expression. Typentetsion. $1998 ; 32: 452-458$.

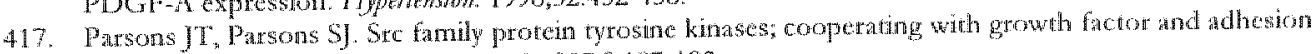
stgnating pathways. Cur Oph CRll Biol 1997;9:187-192.

418. Pasterkamp $G$, De Klein DP, Borst C. Arterial remodeling in aherosclerosis, restenosis and after

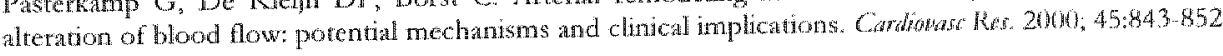


419. Pasterkamp G, Schoneveld AH, Van der Wal AC, Haudenschid CO, Clarijs RJ, Becker AE, Hillen B, Borst C. Relation of atteral geometry to luminal natrowing and histological markers tor plaque vulue rability: the remodeling piatadox. I Ayz Coll Cardol 1998; 32:655-662.

420. Pecrers LLH, Grutrexs $G$, Martin CR Jr. Distribution of cardiac output in the unstressed pregnant guineat pig. Any J Obsted Gyancol 1980;153: $417-425$.

421. Pei D. Weiss SJ. Furrim-dependent intracellular activation of the human stromelysin-3 zymogen. Nathore. $1995 ; 375: 244 \times 247$.

422. Peters $K \mathrm{G}_{\text {w }}$ Vies $\mathrm{CD}$, williams LT, Vascular endothelial growth factor receptor expression during cmbryogenesis and tissue repair suggests a role in endothelial differentiation and blood vessel growth. Proc Noth Alat Si USA 1993,908915-8919.

423. Pfeffer M, Braunwald E, Moye L, Basta L, Brown EJ, Cuddy TE, Dawis BR, Gelmann EM, Goldmaras, Thaker GC, KJein M, Lämas G, Packer M, Rouleau J, Rutherford J, Werthemer JH, Hawkins CM. Eftect of captopril on mortalicy and morbidity in patients with left ventricular dystunction after mpocardial infarction : restuls of the survival and ventricular enlargement trial. $N$ Emg/ J Med. 1992; 327:669-677.

424. Phillips DI, Barker. DJ. Hates CN, Hirst S, Omond C. Thinness at birth and insulin resistance in adult life. Dhidberdofogid. 1994,37:150-154.

425. Pilz RH, Castcel DE fegullation of gene cxpression by cyclic GMP. Cin Re, 2003,93:1034-1046.

426. Poh $U$, Herdan $K$, Huang, $A$, Bassenge $E$. EDRF-mediated shear-induced dilarion opposes myogenic vasoconstriction in small rabbit arteries. Am / P/jysiol. 1991;261:H2016-H2023.

427. Poh U, Hohz J, Busse $\mathbb{R}$, Bassenge E. Crucial role of endohelium in the vasodilation response to increased fow in vivo. Hyporteztsion Dallas. 1986;837-44.

428. Pollman M, Yamada M, Hortuchi M, Gibbons GH. Vasonctive substances regulate wascular smooth muscle cell apoptosis: countervailing influences of nitric axide and angiotensin II. Cre Rer 1996799748756.

429. Popp $\mathbb{R}$, fleming $I_{2}$ Bussie $R$. Pulsate stretch elicits the release of the condothelium-derived hyperpolarizing factor from isolated coronary arteries: a modulator of arterial compliance. Cin Ras. $1998: 82: 696-703$.

430. Portik-Dobos V, Anstad: MP, Hutchinson J, Bannan M, Ergul A. Exidence for a matrix metalloprotcinase induction/activation system in atteral vasculatute and decteased synthesis and acrivation in diabetes. Diaberes. 2002,51:3063-3068.

431. Post MJ, Borst $C$, Pasterkamp $G$. Haudenschild $C C$. Arterial remodeling in atherosclerosis and restenosis: a wague concept of a distinct phenomenon. Atherasteronit. 1995,118:S115-S123.

432. Post MJ, de Smet BJ, wan der Helm Y", Borst C, Kuntz RE. Arterial remodeling after balloon angioplasty or stenting in an atherosderotic experimental model. Cortidtom. 1997,96:996-1003.

433. Poston L., Taylor PD. Glaxo/MRS Young Investigaror Prize. Endothelinm-mediated vascular function in insulin dependent diabetes mellitus. Clin Sa (Lowd). 1995;88:245-255.

434. Pouragend 1: De Mcy JGR. Structural propertes of rat meschteric small atreries a fler 4 -wk exposure to

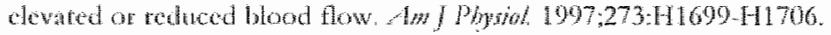

435. Pouragend 1:, De Mcy JGR. Vasomotor responses in cheonically hyperperfused and hypoperfused tast mesenteric anteries, /m P Pyysho 1998;274: H1301-H1307.

436. Pourageand fi. Fites lon Jl. Effects of nitric oxide and sodium nitroprusside on the intrinsic elastic properies of pressurized tat coronary artery. Thuddas Clin Pharmadxd. 1996;10:136-143.

437. Powell JS, Clozel JP, Muller KM. Kuhn H, Heft F, Hosang M, Bamgarmer HR Inhibition of

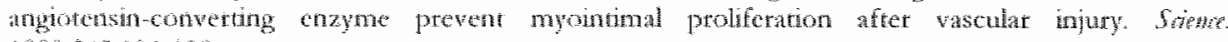
$1989 ; 245: 186 \cdot 138$.

438. Prescot: MII; Webb RL, Reidy MA. Angiotensin converting enzyme inhibitor versus angiotensin M, AT receptor antagonist. Effects on smooth muscle cell migration and proliferstion after balloon catheter

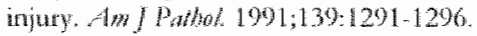

439. Prewitt RL, Rice DC, Dobran AD. Adatation of resistance arreries to increases in pressufe. Mismiryolation, $2002 ; 9295.304$.

446. Price RJ. Skalak "TC. Circumferential wall stress as a mechanism for arteriolat rarefaction and protiferation in a nework model. Whomatsic Res. 1994:47:188,202.

44. Rabinovich SE, Lobarevi LS, Pashlina TS. Purification and some physico-chemical and enzyrnatic

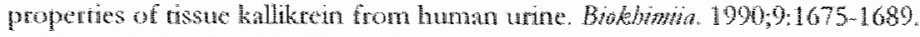

442. Rabinovirch M, Gamble W, Nadas AS, Mietrinen OS, Reid L. Rat pullnonary circulation affex chronic hypoxial hemodynamic and stractural features. Am / Physol 1979;236:H818-H827. 


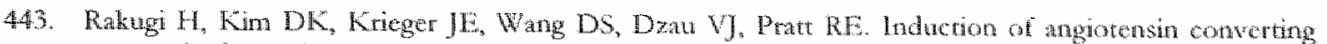

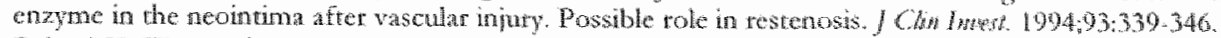

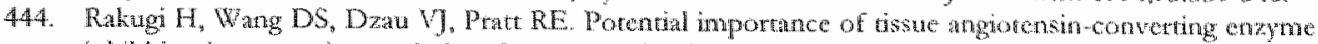

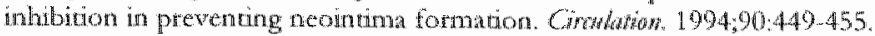

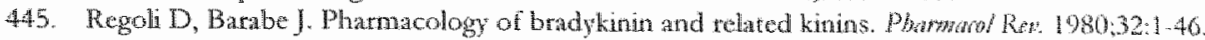

446. Reid MR. Abnomal arteriovenous communcations, acgured and congenital th. The effec of

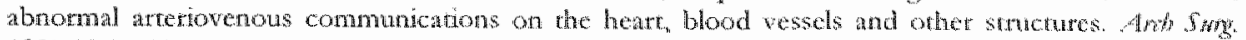
$1925 ; 11: 25-42$.

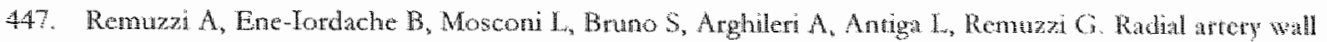
shear stress evaluation in parents with arteriovenous astula for hemodialysis accoss. Bowhotgy. $2003,40: 423-430$

448. Resnick M, Collins T, Atkinson W, Bonthon DT, Dewey CF Jr. Gimbione MA Jr. Platelefderived

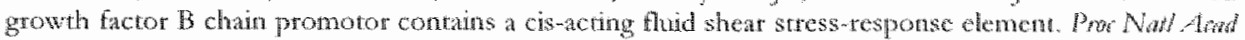
So. $1993,904591-45 \% 5$

440. Reswick $N$, Wahav H, Khachigian LM, Collins "T, Anderson KR, Dewey CF Jro, Gimbronc MA Jr. Endothelial gene regulation by laminar shear stress. Aft Exp Med Bal 1997;430:155-164.

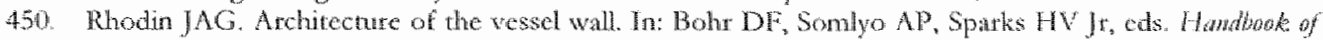

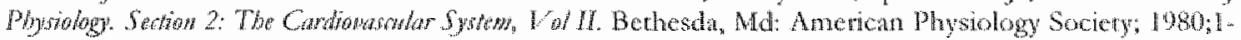
31 .

451. Rieder MJ, Carmona R, Krieger JE, Pritchand KA Jr, Greene AS. Suppression of angionensin-onenting enzyme expression and activity by shert stess. Cin Ros. 1997;80:312 319

452. Riezebos I, Veeming W, Beems RB, Van Amsterdan JGC, Meijer GW, De Wild DJ, Porsius $M$ l,

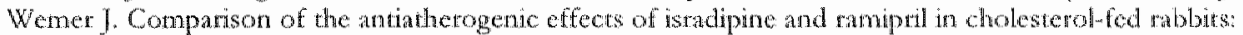
17. effoct on tegtession of atherosclerosis and restoration of endothelial dystunction. I Carbonat Pharyolol. 1994:23:424-431.

453. Rizzoni D, Porteri E, Piocoli A, Castellano M, Betoni G, Muescan ML, Pisini Gi, Guela D, Mulvany M). Rosei EA. Effects of losartarn and anapril on small arlery structure in hyportensive rans. Hypeztension 1998;32,305-310.

454. Rosenberg AA, Koehler RC, fones MD Jr. Distribution of cardine output in fetal and neontal lambs with acute respinatory acidosis. Pedin Res. 1984;18:731-735.

455. Rosenfeld CR Distribution of cardiac ouput in ovine pregnancy. Aw / P/gyol 1977.232231-235.

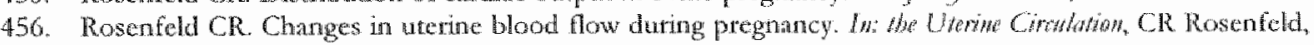
Editor, Ithaca NY. 1989;135-156.

457. Rosenfeld CR, Cox BE, Roy T, Magness RR, Nitric oxde contributes to estrogen-induced vasodilation

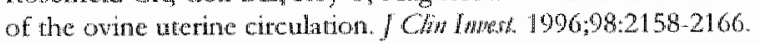

458. Rosenthat SL, Guyton AC. Hemodyamics of collateral vasodilatation following fomonal arery occhsion in anaesdherted dogs. Cin Re. 1068:23:239-248.

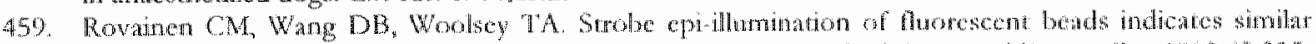

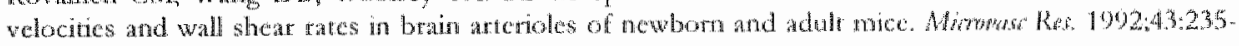
239

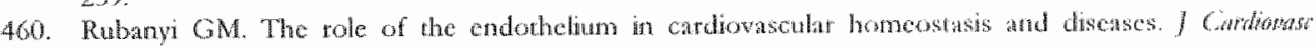
Pbaryack 1903:4:51.514

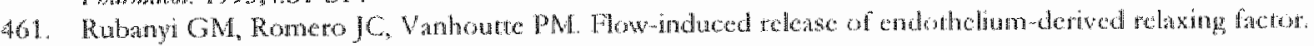
Am J Pbriol $1986,250 \cdot \mathrm{H} 1145-\mathrm{H} 1149$.

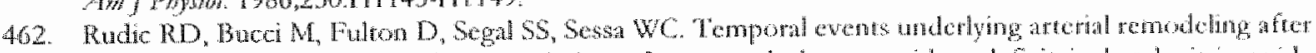

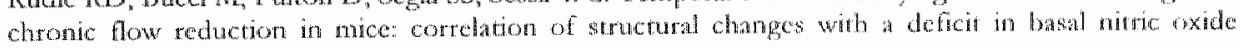
smathesis. Che Rer. 2000;86:1160-1160.

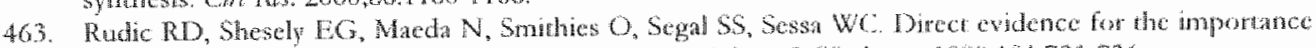

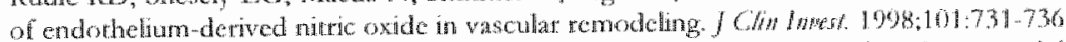

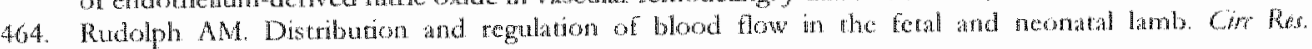
$1985,57.811 .819$

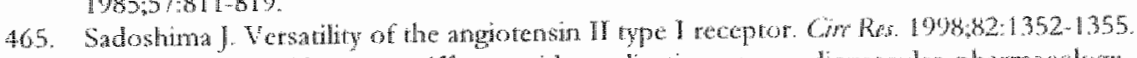

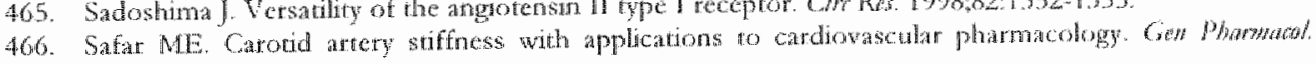
$199627: 1293-1302$

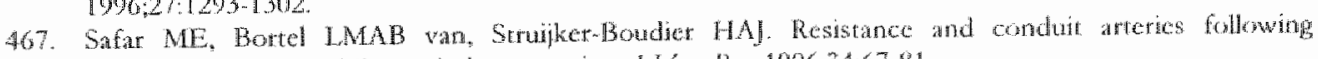

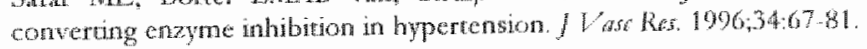




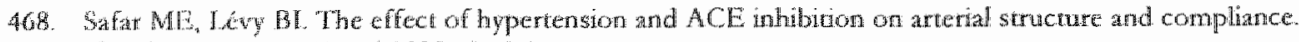

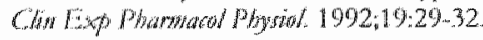

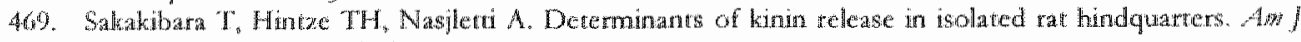
Pysol. $109843: \mathrm{R} 120 \mathrm{R} 125$

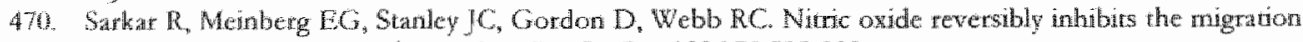
of cullnured vascular smoth muscle cells. Cir Res. 1996;78:225230.

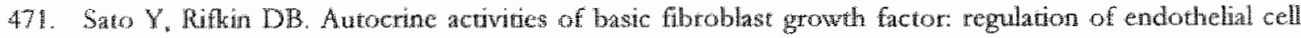

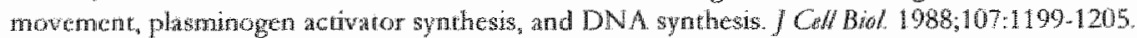

472. Scalbert B, Lewy $B$, Desche P. Devissaguet M, Safar ME. Vascular effects of perindopril: from

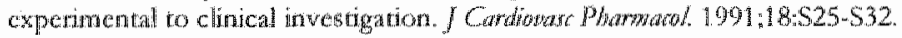

473. Schaper W. New paradigms for collarerat vessel growth. Basi Res Cardoh. 1993,88:193-198.

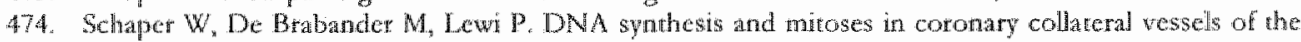
dog. Cin Ret. 1971:28.671.679.

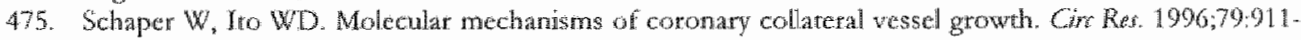
919

476. Sichet DA, Perrone MH. Angiorensin rype 2 receptors mediate dapressor phase of biphasic pressure response to angionersin. Am flysid. $1993,264: \mathrm{R} 917 \mathrm{R} 923$

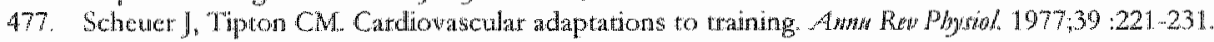

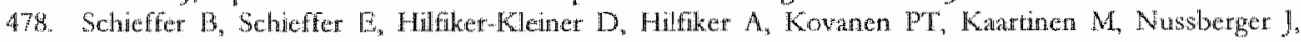
Harringen: W, Dnexler H. Expession of angotensin $\Pi$ and interleukin 6 in luman cononaty

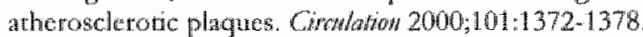

479. Schiffers PMH, Henrion D, Boulanger CM, Colucci-Guyon E, Langa Vuves F, Van Essen H, Fazzi GE, Levy BI, De Mey JGR. Altered fow induced arterial temodeling in vimentin-deficient mice. Antwower

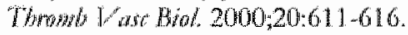

480. Schiffers PMT, Struyker-Bowdier HAJ, De Mey JGR. Fffects of angiotensin Tl and angiotensinconvering enzyme inithitors on contracile and growth responses in isolnted carotid arteries of the rat. Batid Ras Cardol 1991,8683-89.

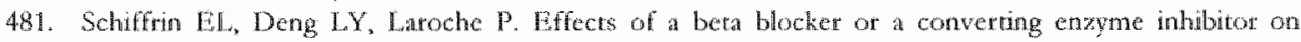
resistance arteries in essential hypertension. Hyertentom 1994:23:83-91.

482. Schatafer DD, Hunter T. Integrin signaling and yrosine phosphorylarion: just the FAKS? Trod, Go/l Biol $1908 ; 8: 151-157$.

483. Schmaict A, Kuo A, Lundberg D, Nurtay S, Cines D. The expression of high moleculat weigh

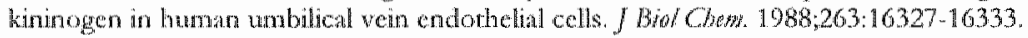

484. Schndi-Trucksäss A, Schmid A, Brumer C, Scheren $N$, Zäh G, Keul J, Huonker M. Artetial properties

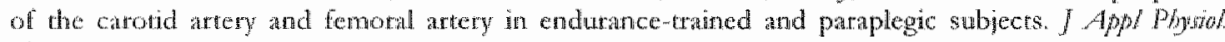
$2000,8 \%: 1956-1963$

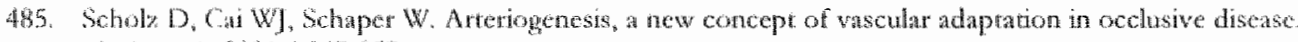
Angrogentir. $2001: 4: 247-257$

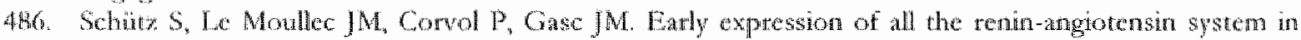

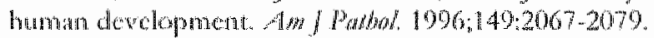

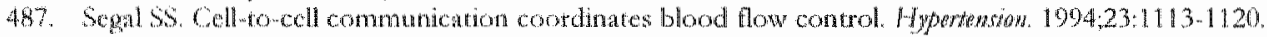

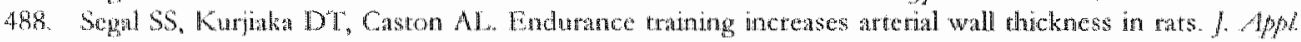
Pojitiol $1093,74: 722,726$

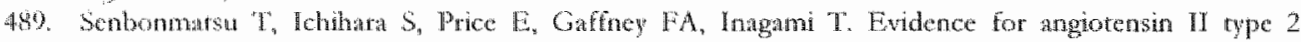

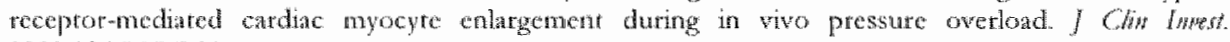
$200 ; 0 ; 06: R 25-1229$.

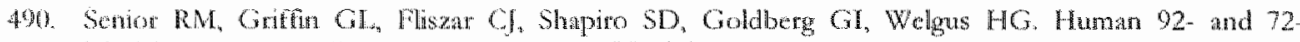

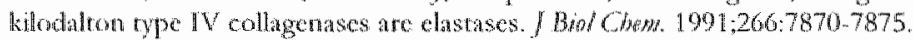

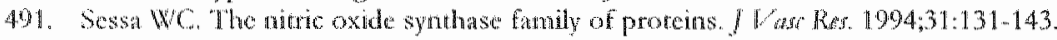

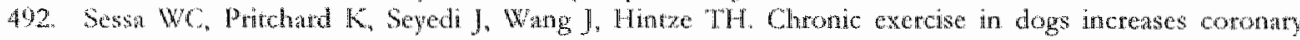

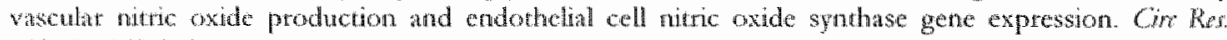
$199474349-353$

493. Seyed $N$, Xu X, Nasjleti A, Hinta "TH Coronary kiningeneration madiates nitric oxide release after

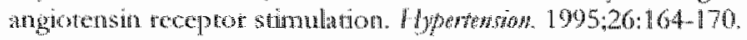

494. Shen I. Luscinskas. FW, Connoly A, Dewey CF Jr, Gimbrone MA Jr. Müd shear stress modulates

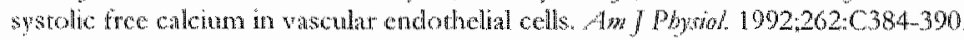


495. Shenk WG, Maran $J W$, Leslie $M B$, Porrin $B A$. The regional hemodynamics of chronic expermenal

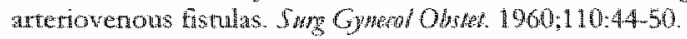

496. Shi $\mathrm{X}$, O'Brica JE, Ala-Kokko L, Chung W, Mannion JD, Zalewski A. Origin of extracellular matrix synhesis during coronary repair. Cirzulawion. 1997,95:997-1006.

497. Shichir $M$, Marumo $F$. Hirat $Y$. Endothelin-B receptor-mediated suppression of endothelial apoprosis. f Cardionstis: Pharmicol. 1998;31:S126-\$27.

498. Sho E, Sho M, Singh TM, Xu C, Zarins $C K$ Masuda H. Blood Alow decrease induces apoptosis of endothelial cells in previously dilated arteries resulcing from chronic high blood flow. Arterooder Thombs Vost Biol. 200121:1139-1145.

499. Shyy JY, Chien S. Role of untegrins in endohelal mechanosensing of shear stress. Cim Rur 2002;91769775 .

500. Shyy JY, Lin MC, Han I, Hu Y, Perrime M, Chien S. The cis-acting phorbol ester "12-Otetradecanoylphorbol 13-acerate"-tresponse element is involved in shear stress-induced monocyte chemotactic protein 1 gene expression. Pro Nat Arab 5e US A. 1995,92:8069-8073.

501. Silvestre IS, Bergaya S, Tamatat R, Duriez M, Boulanger CM, Lery BI. Proangiogenic effect of angiotensin-converting enzyme inhibition is mediated by the bradykinin $B(2)$ receptor pathxay. Cir Res. 2001:89:678-683.

502. Singh $\mathbb{R}$, Pan S, Mueske CS, Witt T, Kleppe LS, Peterson TE, Slobodowa A, Chang JY, Caplice NM, Sumari RD. Role for tissue factor pathway in murine model of wasculat temodeling. Gir Res 2001:89.71 76.

503. Singh $\mathrm{T}$, Abe $\mathrm{K}$, Sasaki $\mathrm{T}$, Zhuang $\mathrm{Y}$, Masuda $\mathrm{H}$, Zarins $\mathrm{C}$. Basic Glbroblast growth factor expression precedes flow-induced arterial enlargement. J $5 \mathrm{~mm}$ Res. 1998;77:165m173.

504. Siragy HM, Inagami T, Ichiki T, Carey RM. Sustaned hypersensitivity to angiotensin III and its

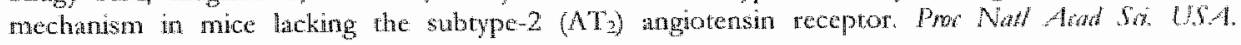
1999:99:6506-6510.

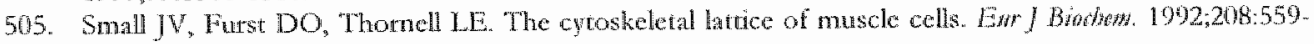
572.

506. Smiesko $\mathrm{V}$. Johnson PC. The arterial lumen is controlled by flow-related shear stress. Nom Pbyot Sor. 1993,8:34 38.

507. Snniesko $\mathrm{V}$, Lang DJ, Johnson PC. Dillacor response of at mesenteric arcading atterioles to increased blood flow velocicy. Am J Pbysing. 1989,257:H1958-H1965.

508. Smith JC, Walters WA, Spong JE. Effects of pregnancy on the diameter of the uterine arteries in the rabbit. Obstet Gymenl 196831:788-794.

509. Song K, Kurobe $\mathrm{Y}$, Kanchara $\mathrm{H}$, Wada T, Inada $\mathrm{Y}$, Nishikawa $\mathrm{K}$, Miyazaki M. Mapping of angiotensin Il receptor subypes in peripheral tissues of spontaneously hypertensive rats by autoradiography. Clin

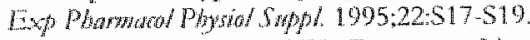

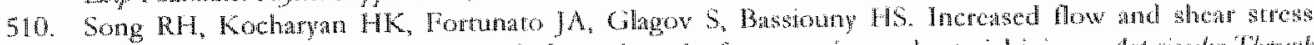

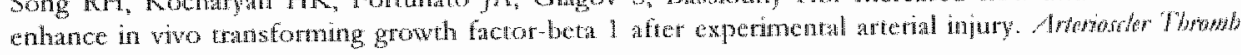
Fas Biol 2000,20023-930.

511. Sonntag WE, Lynch CD, Cooney PT, Hutehins PM. Decteases in cerebral micrevasculature with age and

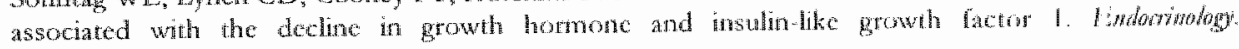
$1097 ; 138: 3515-3520$.

512. Southgate KM, Davies M, Booth RF. Newlyy AC. Involvenwent of extmocllular marrix dograding metalloproteinases in rabbit aortic smooth-muscle cell probiferation. Biodiem /. 1992:288:93.99.

513. Sassen FRM, Raat NJH, Brouwers-Celler DI, Fazi GE, Smits JPM. De Mey fork. Angietensin In

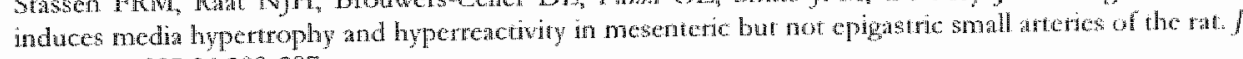
Vasc Res. 1,997;34:289-207.

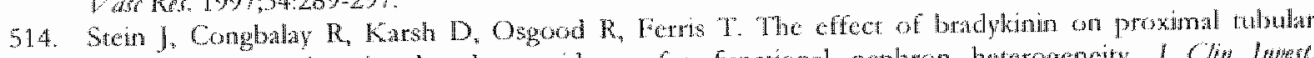
sodium resabsorption in the dog: evidence for functional neplaron hetcrogencity. I Clin linterts. $1972,51: 1709-1721$

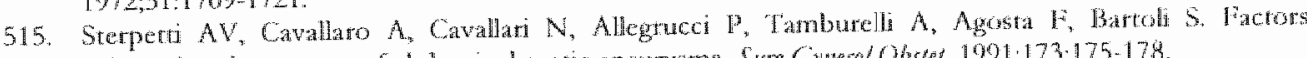
infuencing the rupture of abdominal artic ancurysms. Sarg Gymerol Obster 1901:173:175-178.

516. Serenson E, Daw K, Scals D. Hemoscatic, metallolic, and androggenic risk factors for coronary heart disease in physically active and less active positmenopausal women. Arteribifier Thrombly Vare Biol. $1995: 15: 669-677$. 


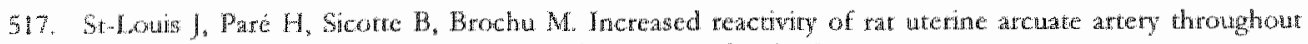

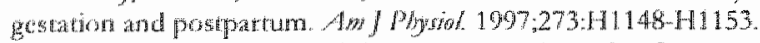

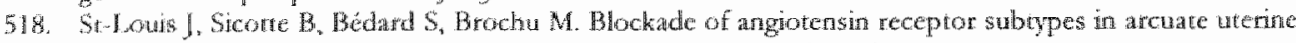

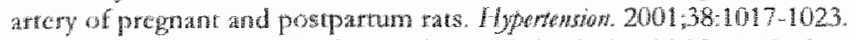

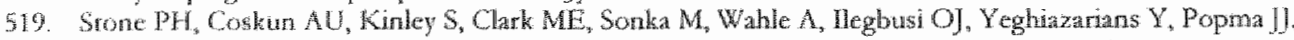
Oray 1 , Kunty RE, Reldman CL. Eftect of endothelial shear stress on the progression of coronary artery discase, wacular remodeling, and in-satent testenosis in hamans. In vivo 6-month followw study. Cirmhinow. 2003; $108: 438444$

52. Stoment JM, Meye M, Osol G. Bbtrogen augments the vasoditatory effects of vascular erdorhelial

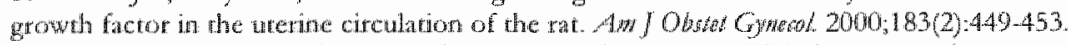

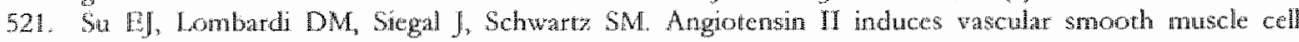
replication independent of blood pessurt. Hyperterion. $1908,31: 13311-1337$.

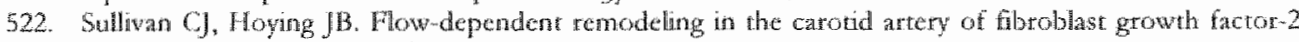

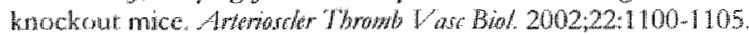

523. Sun D, Hewng $A$, Koller $A$, Kaley G. Short tem daly exercise activity enlances endohelial NO symthesis in sketetal muscle arterioles of rats. $\int$ App Pbyod $1994,76: 2241-2247$.

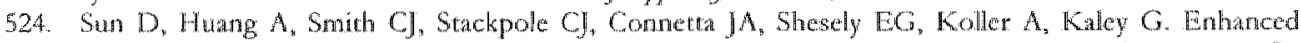
release of prostaglandins contributes to flow-joluced atreriolat dianon in wOS knockout mice. Cm Rer. $1999.85: 288-293$.

525. Sun D, Huang A, Yan GH, Wu Z, Yan C, Kamineki PM. Oury TD, Wolin MS, Kalley G. Redaced nelease of mitric oxide to shent stress in mesenteric arteries of aged rats. 4 mo J Poysol. 2004:1n Prest.

526. Srension A. Hypertension in pregnancy: stete of the art lecture. / Hoprom. 1085;3:5395-5403

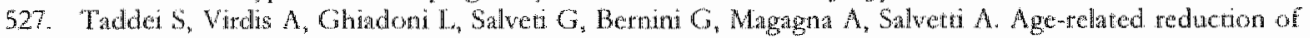
NO avalabilicy and oxidative stess in humans. Hypertersion. 2001;38:274-279.

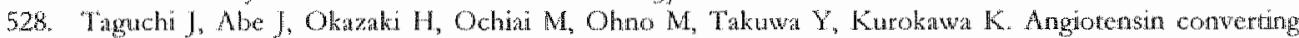
ensyme inhibitors or Dup 753 prewent neomtimal formaton following balloon injury win simgle topical

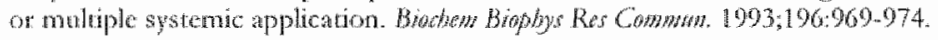

520. Tai LK, Okuda M, Abe J, Yan C, Benk BC. Hlud shear stress actwares proline-rich tyrosine kinase via

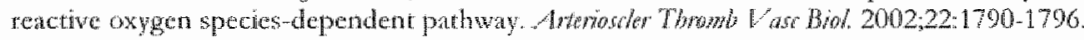

530. Tanaka M, Tsuchida S, Ima T, Fuji N, Mitadki H, Ichiki T, Naruse M, Inagami T. Vascular response ro

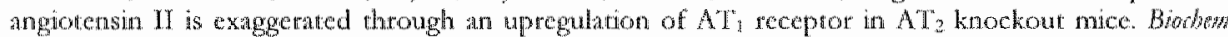
Brophys Riss Commm. 1909:258:194-198.

531. Taniyama $Y$, Griendling KK. Reactive oxygen species in the rasculature: moleculat and calluar mechanisns. Hypertowion. 2003;42:1075-1081.

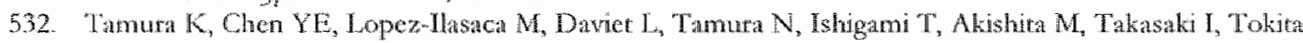

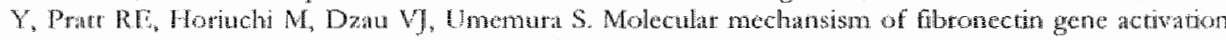

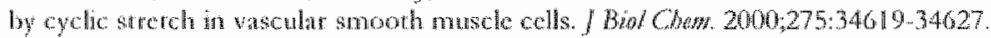

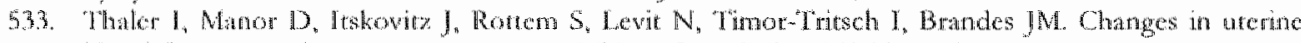

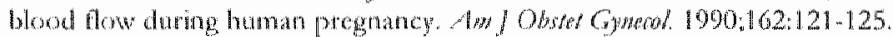

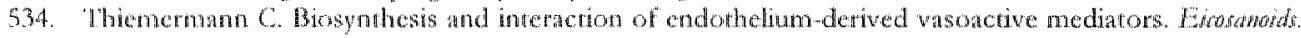
$1091+197-202$

535. Thoma R. Wrorsuchungen uber de listogenese und hiscomochank des Gefassystems (Enke, Sarthat);

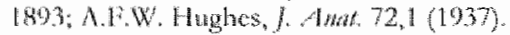

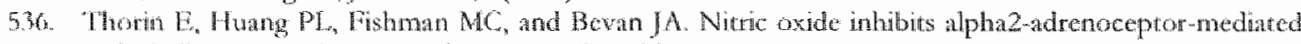

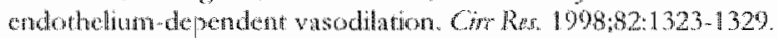

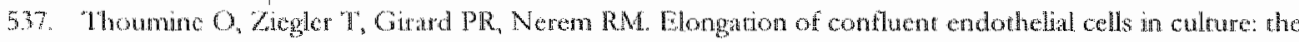

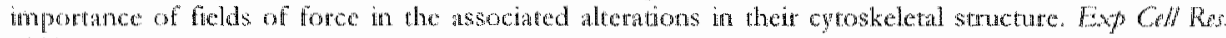
1095,$210 ; 427,441$

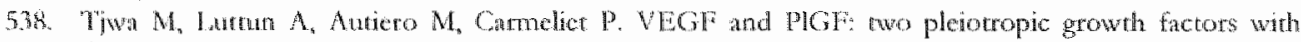

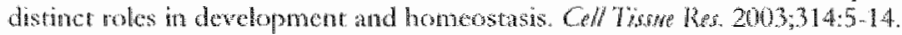

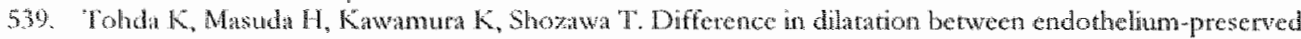

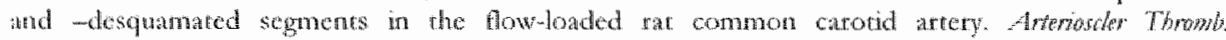
$1992.12 .519-528$

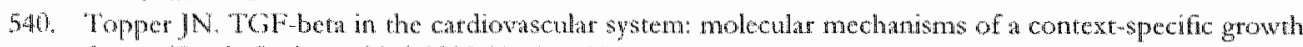

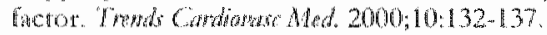


541. Topper JN, Cai J, Falb D, Grimbone MA Jr. Identification of vascular endorhelial genes differentially responsive to fluid mechanical stimuli: cyclooxgenase 2, manganese superoxide dismutase, and

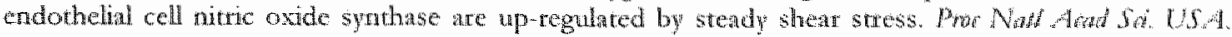
$1996 ; 93: 10417-10422$.

542. Topper IN, Gimbrone MA Jr. Blood fow and wascular gene exptession: flud shear stress as a modutaror of endothelial phenotype. Mor Mod Todidy 1999,5:40-46.

543. Touyz RM, Schiffrin EL. Angiotensin II reguates vascular smooth muscle cell $\mathrm{pH}$ t contraction and growth via tyrosine-kinase dependen signaling pathways. Hyperten:son 1907,30:222-229.

544. Tranb O, Berk BC. Laminar shear stress: mechanisms by which endothelial cells transduce an atheroptotective force. Arerioster Thromb V an Biol, 1998;18:677-685

545. Tribe RM, Thomas CR, Poston L. Flow-induced diatation in isolated resistance atteries from control and streptozotocin diaberic rats. Diabetologit. 1998; 41:34-39.

546. Tronc F, Mallat Z, Lehoux S, Wassa $\mathrm{A}$, Esposito B. Tedgui A Role of matrix metalloproteinses in blood flow-induced atterial enlargement: Interaction with $\mathrm{NO}$. Arteriosder Tbram $\mathrm{F}$ abr Biot. $2000 ; 20: 0120-\mathrm{e} 126$

547. Tronc E. Wassef M, Esposito B, Hennion D, Glagow S, Tedgui A. Role of NO on flow-induced

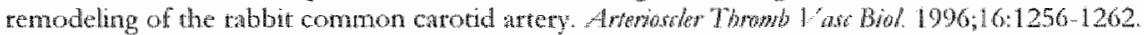

548. T'schudi MR, Barton M, Bersinger NA, Moreau P, Cosentino F, Noll G, Malinski T", Luischer TF. Effects of age on kinetics of nitric oxide release in rat aorta and pulmonary arery. J Chin Itwest 1996;98:899-905.

549. Tschudi MR, Lüscher TF. Age and hypertension differentially affec coronary contractions to endothelin-1, serotonin, and angiotensins. Chatatioss. 1995:91:2415-2422.

550. Trsuda $Y$, Olazaki $M$, Uezono $Y$, Osaima $\Lambda$, Kato $H$, Olada $H$, Oishi $Y$, Yashiro A, Nakashima $Y$ Activarion of extracellular signal-regulated kinases is essential for pressurc-induced proliferation of vascular smooth muscle cells. Eur / Phatpolot. 2002;446:15-24.

551. Tsutsumi $Y$, Matsubata H, Masaki H, Kurihara H, Murasawa S, Takai S, Myazalki M, Nozawa Y, Ozono $\mathbb{R}$, Nakagawa $\mathbb{K}$, Mwa $T$, Kawada $N$, Mori $Y$, Shibasaki $Y$, Tamaka $Y$, Fuilyama S, Koyama $Y$, Fuilyana A, Takahasht $H$, Iwasali $T$. Angiotensin II type 2 receptor overexpression ativates the vascular kinin system and causes wasodilation, $J$ Chn Inrest. 1090;104:525-935.

552. Tulis DA, Prewitt RL. Medial and endothelial platelet-derived growh factor A chin expression is regulated by in wivo exposure to elevated flow. J Vase Res. 1998;35:413-420.

553. Tulis DA, Unthank JL, Prewitt RI. Flow-induced remodeling in rat mesenteric vasculature. Am f Physiof. $1998,274:-1-1874-\mathrm{H} 1882$.

554. Tuttle JL, Hahn TL. Sanders BM, Wirman FA, Miller SI, Dalsing MC, Unthank JL Impaired collateral development in mature rats. Am J Pbysol. 2002;283-H146-H155.

555. Turtle JL, Nachreiner RD, Bhuller AS, Condict KW, Connors BA, Herring BP, Dalsing MC, Unthank

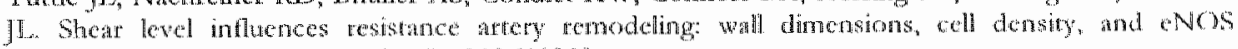
expression. Amy J. Pbysiol. 2001,281:H1380-H1389.

556. Unthank ]L, Fath SW, Burkhart HM, Miller S, Dalsing MC. Wall remodeling during luminal axpansion of mesenteric arterial collatetals in the rat. Cim Res. 199679:1015-1023.

557. Unthank JIs, Nixon JC Burlkhart HM, Fath SW, Dalsing MC. Garly collateral and microwasculat

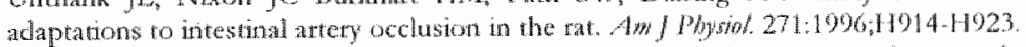

558. Unthank $\rrbracket \mathrm{L}$, Nixon $J \mathrm{C}$, Lash $J \mathrm{M}$. Barly adaptations in collatatal and microvascular resistances after ligation of the rat femoral artery. J App Pbysit. 1995;70:73-82

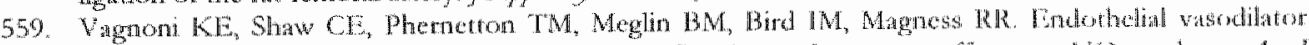
production by utcriace and systemic arteries, HI. Ovarian and estrogen afocts on No synthase. Am I Plyitol. 1908,275:H1845-11856.

560. Valdes $G$, Germain $A M$, Corthorn J, Chacon C, Figueroa CD, Muller-Lsterl w, Tissuc kalliken and bradykinin $B_{2}$ receptor in human utcrus in futeal phase and in carly and late gestation. Lindacring 2001; 16(3):207-215.

561. Wan der Heijen OWH, Essers YPG, Simken LHI, Teunissen QGA, Peeters LHW De Mey JGR, Van Eys GJJM. Aging blunts remodeling of the uterine artery during murine pregnancy, In Pres 2004.

502. Van der Loo B, Labugger R, Skepper IN, Bachsehmid M, Kilo J, Powrill JM, Palacios-Callender M,

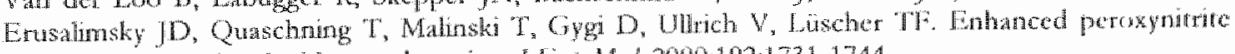
formation is associated wh th vascular aging. J Exp Med 2000,192:1731-1744.

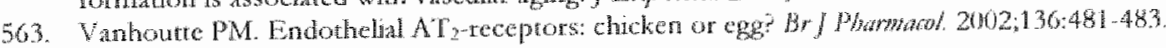


564. Van Kandik o, Pewers LL. Clncal appers of pregnancy after the age of 35 years. a teview of the

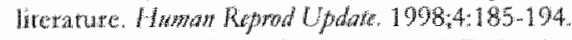

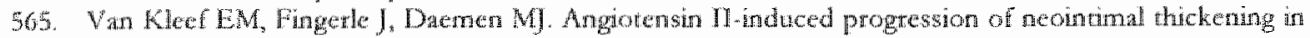

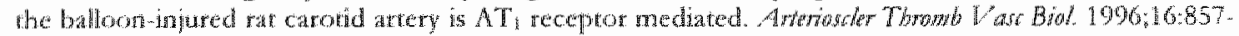
863.

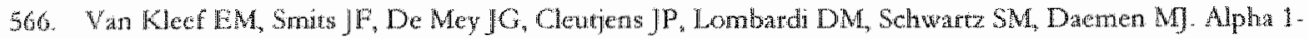
adrenocepiar blockade reduces the angiotensin II-induced wascular smooth muscle cell DNA gymesis

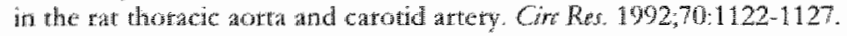

567. Van Royen W, Piak JJ, Schaper W, Bode C, Buschmamn I. Arteriogetacis: mechanisms and modularion of collateral attery development. I Nut Cardot 2001;8:687-693

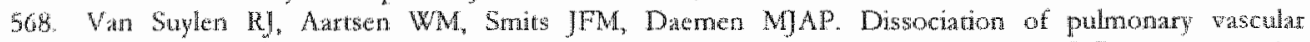

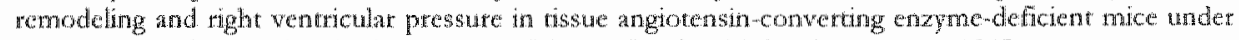
conditions of chronic alveolar hypoxia. Am if Regit Chit Care Med 2001; $63: 1241-1245$.

569. Vassalle $C$, Lubrano $V$, Domenici $C$, L Abbate $A$. Infuence of chtonic aerobic exercise on microctrculatory Dow and nitric oxide in lumans. In / Sport Med 2003,24:30-35.

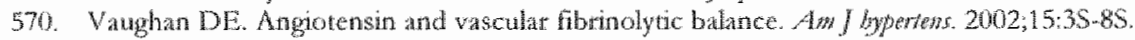

571. Verareddy S, Cooke CLM, Baker M, Dawdge ST. Vascular adaprations to pregnancy in mice: erects on myogenic lone. Aw J Pysial. 2002;283:H2226- 12233 .

572. Venema RC, Sayegh HS, Amal J.F, Hatrison DG. Role of eazme calmodulin-binding domain in

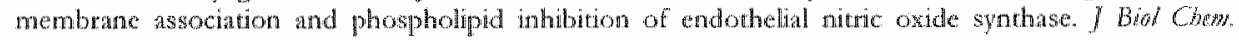
$1995270 \% 14705-14711$

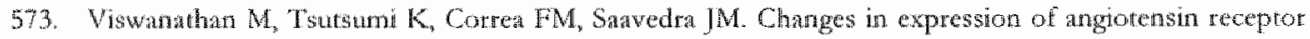

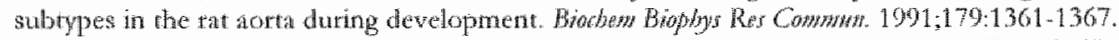

574. Von der Lewen HE, Gibbons GH, Morghta $R$, Lewis NP, Zhang L, Nakaima M, Kancda Y, Cooko JP, Dzau VJ. Gene therpy inhbing neomtimal wasular lesion: in vivo ransfer of endothelial nitric oxide syruthase genc. Prow Nal Aad So 1995,92:1137-1141.275.

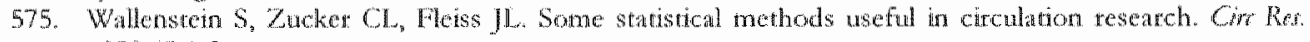
$1980,47: 1-9$

576. Whither T, Menrad A, Orachowski HD. Siemester $G$, Pan M, Schirner M. Differential regulation of in wivo angiogenesis by atugiotensin III receptars. FASEB /. 2003;17:2061-2067.

577. Wang, DB, Blochet NC, spence ME, Rovanen CM, Woolsoy "TA. Development and renodeling of cerebral blood vessels and their fow in postnatal mice observed with in vivo videomicroscopy. $J$ Cen Blowd low Wow $1992,12.935-946$.

578. Wnng DH, Prewir RL. Mictowascular dwelopment dung normal growth and teduced biood Alow: introduction of a new model. Am Pbyral 1991260:H1966-H1972.

579. Wang J, Wolin MS. Hintze TH. Chronic exercise enhances endorhelum-mediated datarion of epicardial coronaty artery in conscious dogs. Cor Rer. $1993,73.829 .838$

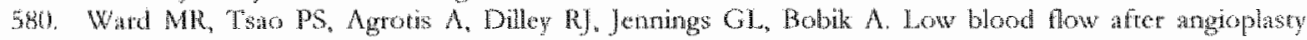
athgrents mechnisms of restenosis. Inwat vesel remodeling, cell migration, and actity of genes

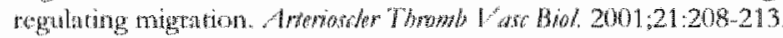

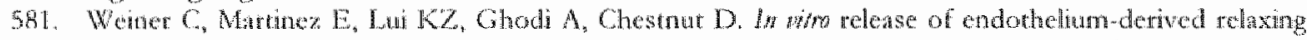

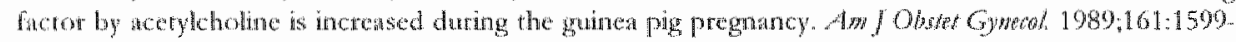
1005

582. Weinet CP, Lizasonan 1, Baylis SA, Knowles RG, Chates $1 G$, Moncada S. Induction of calciun-

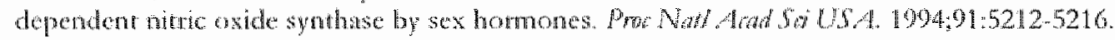

583, We1s SM, Langile BH, Adamson SE. In who and in vitro mechancal propertics of the sheep thoractic

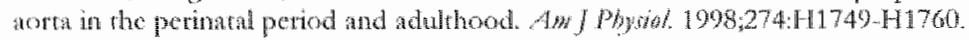

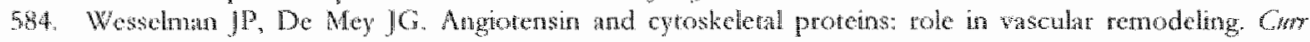

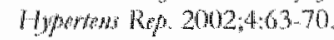

585. Wesschna JP, Dobtan AD, Shriver SD, Prewite RL. Sre tyrosine kinases and extracellatar signalrelnted kinase $1 / 2$ mitogen-acrivated protoin kinases mediate pressure-induced c-tow expression in

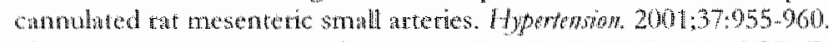

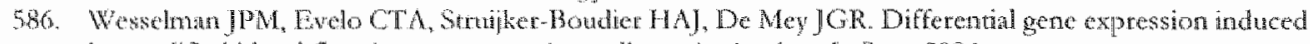
by modified blood tow in tat mesenteric small arteries in vivo in prot. 2004

587. Wiemer G, Scholkne BA, becke RHA, Busse R. Ramipilat enhances endothelal autocoid formation

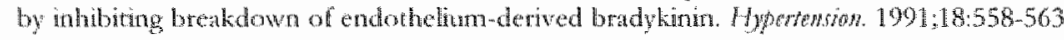


588. Wiemer $\mathrm{G}$, Scholkens BA, Wagner A, Heirsh H, Linz W. The functional role of angiotensin If subtype

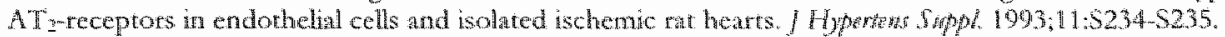

589. Wiinen J, Kuipers H, Kool M, Hoeks A, wan Bakk M, Struijker-Boudter H, Verstappen F, Van Bortel L.

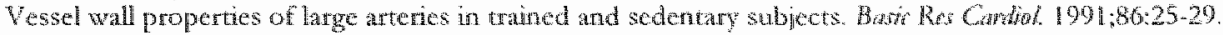

590. Wilson DD, De Garavilla L, Kuhn W, Togo J. Burch RM, Stenanka U. D. Atg [Hyp 3DPhe7] bradykinin, a bradykinin antagonist, decreases mortality in a rat model of endotoxic shock. Cim, Shock 1089;27,93. 101.

591. Wolf C, Cai W], Vosschulte R, Katai S, Mousawipour D, Scholz D, Arsh-Hediri A, Schaper W, Schaper J. Vascular remodeling and altered protein expression during growth of coronary collateral arteries./ Mol Cell Candiol $1998 ; 30: 22912305$.

592. Wolin MS, Gupte SA, Oeckler RA. Superoxide in the voseular syssem / War Re, 2002:39:191-207.

593. Wong AHY, Kulandavelu S, Whiteley KJ, Qu D. Lowell Langille B. Lec Adamson S. Maternal cardiovascular changes during pregnancy and postpartum in mice. Afos / Phyol 2002;282:14918-4925.

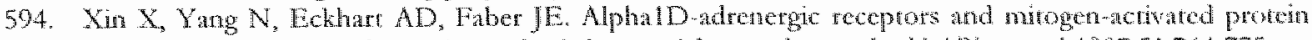
kinase mediate increased protein synthesis by arteral smooth musele. Mo/ Pharatrol. 1907,51:764-775.

595. Xu C, Jee $\mathrm{S}$, Shu $\mathrm{C}$, Masuda H, Zarins $\mathrm{CK}$. Expression of TGF $\beta 31$ and $\beta 3$ but not apoptosis factors

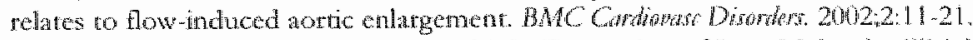

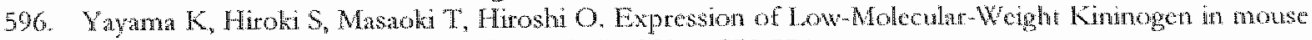
Wascular smooth muscle cells. Brof Pham Bant. 1908:21:772774.

597. Yanagisawa M, Kurihata H, Kirnura S, Tomobe Y, Kobayashi M, Mitsui Y, Yazaki Y, Goto K, Masaki T. A novel potent wasoconstrictor peptide produced by wasculat endothelial cells. Natum. 1988,332,411 415.

598. Yogo K, Shimokawa $H$, Funakosh $H$, Kandabash $T$, Myata K, Okamoro S, Egashira K, Huang P, Akaike T, Takeshita A. Different wasculoprorective roles of NO synthase isoforms in vascular lesion

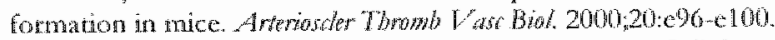

599. Zahradka $\mathrm{P}$, Wilson D, Saward L, Yau L, Chenng PK. Cellular phyciology of augionensin 11 recepturs in

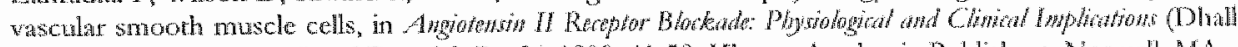
NS, Zathradka P, Dixon I and Beamish R eds, 1998: 41-50, Kluwer Academic Publishers, Nonwell, MA.

600. Zalewski $A$, Shi $Y$. Vascular myotibroblass: lessons from cononary repatr and remodeling Anforionder Thromb Vow Brol. 1997;17:417-422.

601. Zarins $C K$, Zatina MA. Giddens DP, Ku DN, Gligow S. Shear stress regulation of artery lumen diameter in experimental atherogenesis. I Vost Surg. 1987,5:413-420.

602. Zeiher AM, Drexler $\mathrm{H}$, Wollschlager $H$, Just $H$. Endothelial dysfuncrion of the coronary microvasculature is associated with impaired cotonaty blood flow regulation in patients with early atherosclerosis. Cinmiation. 1991;84:1984-1992.

603. Zeiher AM, Drexler H, Sautbier B, Just H. Endothelium-modiated coronary blood flow modultion in humans: effect of age, atheroselenosis, hypercholesterolemia and hypertension. / Clin Iswest 1993;92:652 602.

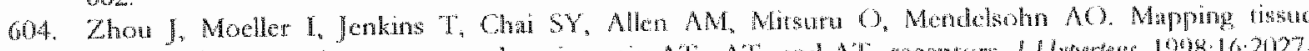

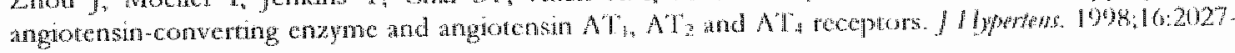
2037.

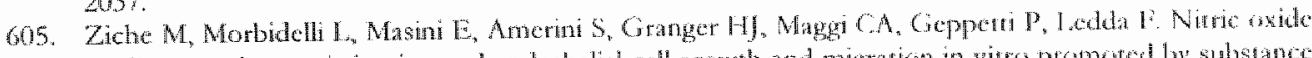

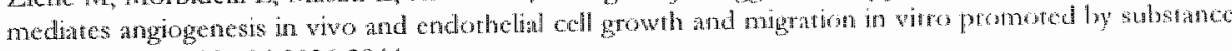

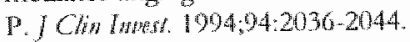

606. Zieglet T, Silicei P, Harrison V], Hayoz D. Nitric oxide synthase cxpression in endothelal cells expessed to mechanical fores. Hypertusion. $1998 ; 32: 351-355$.

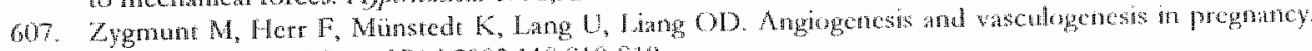

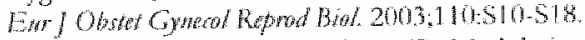

608. Zwart AS, Davis EA. Widdop RE. Modulation of Al, receptomedianced contraction of at uterine artery by $\mathrm{AT}_{2}$ receptors. Brf Plarmold 1098;125:14291436. 



\section{Summary}

Blood vessels have the adaptive mechanism to respond to, and normalize changes in hemodynamic stimuli such as pressure and flow. Situations of disturbed hemodynamic stresses occur in wivo for instance during pathological disorders such as hypertension and athetosclerosis, but also during physiological processes such as exercise and pregnancy. Endothelial cells are directly in contact with the dragging blood flow and are therefore particularly sensitive to changes in shear stress (flow). In the first chapter we described the acute (flow-induced dilatarion) and chronic arterial responses (remodeling) to flow in particular. We focused on the renin-angiotensin system and the kallikrein-kinin system, since the main objective of the present thesis was to determine the role of angiotensin II and bradykinin, two important mediators of both systems, in acute and chronic arterial responses to altered blood flow.

Tissue kallikrein-deficient mice ( $\mathrm{TK} /$ ) were studied to atralyze the effect of an absence of membrane-bound tissue kallikrein (and hence reduced bradykinin synthesis) on acute vasomotor responses and chronic structural responses to flow in both large conduit arteries (carotid arteries) and small peripheral arteries (uterine arteries). In chapter 2 we studied acute flow-induced dilatation in large conduit arteries such as the carotid artery. We confirmed previous observations that flow-induced dilatation was significantly blunted in carotid arteries of TK $/$ mice, demonstrating the key role of TK in acute flowinduced dilatation, by releasing endogenous kinins that act on endothelial bradykinin $B_{2}$ receptors. Furthermore, we demonstrated that this response required functional angiotensin type 2 receptors.

The role of the vascular kallikrein-kinin system during arterial remodeling in response to chronic changes in blood flow was analyzed by using a surgical model. In chapter 3 unilateral left carotid antery ligation was performed in both wild-type and TK mice, which resulted in a complete cessation in blood flow in the ligated left carotid attery and a doubling of blood flow in the contralateral right carotid artery. The vascular kallikrein-kinin system seems to protect against excessive inward temodeling, but was not involved in medial wall mass changes in response to chronic cessations in blood flow.

The participation of the kallikrein-kinin system in acute flow-induced dilatation was also analyzed in small tesistance-sized atteries. Similatly to tesponses in the carotid artery, flow-induced dilatation was blunted in uterine arteries of TK mice (chapter 4). Pregnancy, characterized by a drastic rise in uterine blood flow, resulted in an cnhanced release of nitric oxide in response to shear stress by uterinc arteries of both wild-type and TK - mice. The difference in the maximal vasodilator response to shear stress persisted between wild-rype and $\mathrm{TK} /$ mice. Despire this reduced vasodilator response to shear stress in TK mice, pregnancy-induced urerine artery outward hypertrophic remodeling in response to the uterine blood flow increase, was not compromised. Immediately after birth uterine blood flow returns to normal flow walues, representing a physiological model of reduced blood flow. We observed that hlow-induced dilatation was markedly reduced in uterine arteries of 7 -days postpartum mice, and the arrerial structure regressed toward prepregnant structure, demonstrating the dynamic nature of the uterine vascular bed dusing and after pregnancy. 
Tissue angiotensin-converting enzyme-deficient mice $(\mathrm{t}-\mathrm{ACE}$ ) wete studied to analyze the effect of an absence of membrane-bound tissue $A C E$ (and hence reduced angiotensin II synthesis, but enhanced endogenous bradykinin levels) on acute vasomotor tesponses and chronic structural responses to flow in both carodid and uterine arteries. In chapter 5 we investygated the role of endogenous bradykinin in chronic modulation of endotheliumdependent vascular reactivity that occurs during and after pregnancy. We showed that bradyknin can be released by both chemical (acerylcholine) and rnechanical (shear stress) stimulation of uterine arteries of wild-type and $\mathrm{ACE} / \mathrm{Anice}$. The contribution of bradykin in these vasodilator tesponses was higher in wterine arteries of $t-\mathrm{ACE} / \mathrm{mice}$, since blockade of $B_{2}$ receptors inhibited the response to acetylcholine and shear stress to a greater extent in $t-A C E /$ mice. During pregmancy the contribution of nitric oxide to the shear stress-induced vasodilatation was more substantial in uterine arteries of wild-rype mice compared to $\mathrm{ACE} / \mathrm{ACE}$, whereas the contribution of bradykinin was more pronounced in late pregnatut $\mathrm{ACE} / \mathrm{Amice}$, demonstrating the modulatory role of endothelium-derived vasoactive factors like bradylkinin during flow-induced dilatation of uterine arteties in situations of chronically altered uterine blood flow.

Tissue ACE deficiency resulted in a reduced arterial elasticity (chapter 6 ). This reduced elasticity originated from $B_{2}$ receptor stimulation, since chronic (14 days) blockade of these receptors restored the atterial elasticity in $\mathrm{t}-\mathrm{ACE} / \mathrm{m}$ mice. These data demonstrate the close relationshp between the tenin-angiotensin system and the kallikrein-kinin system and their contribution to the maturation of the cardiovascular system.

In chapter 7 we tested whether tissue ACE is inwolved in arterial remodeling by applying the unilateral carorid antery ligation method and studying urentne arteries before, during and after pregnancy in wild-type and $\mathrm{t}-\mathrm{ACE} \%$ mice. In the ligated carotid artery of t-ACE-/ mice, the observed medial hyperplasia, inward remodeling and arterial distensibility were teduced compared to the wild-type counterpart. Thus, tissue ACE plays a major role in suppressing the hyperplastic inward temodeling of the carotid artery imposed by blood flow cessation. Comparing the results of chapter 3 and 7 , it seems that angorensin II is responsible for the vascular smooth muscle cell hyperplasia, whereas bradykinin prevents luminal shrinkage in response to drastic decteascs in large artery blood flow. Like tissue kalliken, tissue ACE is not essential for the outward hypertrophic and subsequent inward hypotrophic remodeling of the uterine artery during and after pregnancy

In chapter 8 the effects of postponed murine pregrancy on acute and chronic uterine artery responses were investigated. Our findings suggest that at middle age the effecs of shear stress on endothelial nitric oxide synthase phosphorylation and subsequent nitric oxide release are lost. Upregularion by other endothelium detived factors like prostacyclin or endothelium-derved hyperpolarizing factor compensate during acute flow-induced dilatarion, but can not sustain chronic pregnancy-induced arterial remodeling. This might explain the reduced number of viable fetuses in the thind trimester of postponed murine pregnancy.

From the results obtaned in this thesis it is clear that blunted acute arterial tesponses to shear stress do not necessarily entail a defect in the remodeling response afrer chronic alterations in shear stress. Strengthened by our observations and others we have written in 
chapter 9 a review on the supposed dissociation between acute and chronic arterial responses to shear stress.

In summary, the results described in this thesis confirm the beneficial effects of bradykinin as is increasingly suggested by experimental and clinical drug-intervention studies. Furthermote, acute flow-induced vasodilatation and structural flow-induced arterial remodeling involve alternative mechanisms, which opens new possibilities for the development of new targets for cardiovascular-pharmaco therapy. In addition, adaptive structural arterial adaptations to shear stress differ between large elastic and pre-existing collateral arteries, and the effect of even middle age is more marked than deficiency of cardiovascular key enzymes, such as tissue kallikrein or tissue angiotensin-converting enzyme. This raises new perspectives, such as site-directed interventions, for unraveling the effects of aging and regionality on arterial remodeling. 


\section{Samenvatting}

Bloedvaten hebben de adaptieve eigenschap om te reageren op verandetingen in hemodynamische stimuli zoals druk en stroming (kortweg flow). Processen ran een verstoorde hemodynamische balans kumen zich win wo vootoen bij parhologische aandoeningen zoals Jypertensie en atheroscletose, maar ook tijdens fysiologische processen zoals langdurige lichamelike inspanning en de zwangerschap. Endotheelcellen zijn in direct contact met het stromende bloed en zijn dastoor bijzonder grevoelig voor veranderingen in afschuifspanning of flow. In hoofdstuk 1 beschriven we de acute (flowgeinduceerde vaatverwijding) en chronische responsen (remodelering) op flow. Hierbij hebben we ons gericht op de rol wan angiotensine II en bradykinine, twee belangrijke mediatoren van respectievelijk het renine-angiotensine systecm en het kallikreine-kinine systeem, bij de acute en chronische arteriele responsen op een veranderde flow.

Weefsel kallikreine-deficiënte muizen $\left(\mathrm{TK}^{\%}\right)$ werden bestudeerd om het effect van de afwezigheid van membraan-gebonden weefsel kalikreine (en dus een verminderde bradylinine synthese) op acute vasomotor tesponsen en chronische stucturele responsen op flow te analyzeren in zowel grote arterien (carotide arterièn) en kleine perifere arterien (uteriene arterien). In hoofdstuk 2 hebben we de acute door flow-geinduceerde vatwerwijding in de carotide arterie onderzocht. We bevestigden eerdere resulaten dat de door flow-geinduceetde vaatverwijding significant verlaggd is in carotide artetiën van TK - muizen, hetgeen de cruciale rol van weefsel lkalikreine in acute door flow-geinduceerde warwerwijding atatoont, via de secretie van endogene kinines die arangripen op endotheliale bradylinime $B_{2}$ receptoren. Bovendien toonden we aan dat deze reactic afhankelijk is van functionele angiotensine type 2 receptoren.

Een chinurgisch model werd toegepast om de rol van het vasculaite kallikreine-kinine systeem bij arterible remodelering tijdens chronische flow veranderingen te bestuderen. In hoofdstuk 3 pasten we unilaterale ligatie van de linker carotide arterie toe op zowel wildcype als TK- muizen, hetgeen resulteende in een complete obstructie van de flow door dit afgebonden bloedvat en een verdubbeling van de flow in de rechter catotide atterie. Het vasculaire kallikreme-kinine system bleek te beschemen tegen ovematige inwardse remodelering, matr was niet betrokken bij mediale watwandmassa veranderingen in respons op chronische flow cessatics.

Een bijdrage van het kallikreine-kinine system in acute llow-geinducerde vatwerwijding werd tevens onderzocht in keine weerstandsarterièn. In analogie met do responsen in de carotide arterie, was de doot flow-genducende vaatwerwijing verlaggd in uteriene arterien van $T K$ muzen (hoofdstuk 4). De zwangerschap, die gekarakteriseerd wordt doot een drastische toename in de doorloloding van het uteriene vatbed, resulteerde in een verhoogde ptoductie van stikstofmonooxide doot de uteriene arterie van wild-type en TK/ muzen in teactic op een verhoogde afschuifspanning op de endotheeleellen. Het verschil in de maximale varverwijdende respons op deze afschuifspanning was onveranderd tussen wild-type en $T K /$ murzen. Ondanks deze verminderde vaarverwijdende respons in TK muizen, was de doot zwangerschapgeinduceede uitwaardse hypertofe temodelering van de uteriene arterie ten gevolge wan de verhoogde dootbloeding van het uteriene vatbed identick an dic van wild-type muizen. Kont na de geboote daat de doorbloeding van het uteriene vatubed tot nomale 
warden, hetgeen een fysiologisch model voor een verminderde flow simuleert. We toonden aan dat de acute door flow-geinduceerde vatvenwijing in uteriene attetien van 7 dagen postpartum muzen drastisch is gereduceerd en dat de varwand dimensies van de niet-zwangere toestand terugkexrde.

Weefsel angiotensine-converting enzyme-deficiënte (t-ACE $/$ ) muizen werden bestudeerd om de gevolgen van het gemis van membraati-gebonden weefsel $A C E$ (en dientengevolge een verlagde angiotensime II synthese, maar tevens verhoogde endogene bradykinine spiegels) we bepalen op acute vasomotor responsen en chronische structurele responsen op how in zowel carotide als uteriene atterién. De rol van endogeen bradykinine bij de chronische modulatie van endotheel-afhankelike vatwand reactivirert, die opreedr tidens en na de zwangerschap, werd onderzocht in hoofdstuk 5. We licten zien dat bradykinine kan worden vrijgemaakt doot zowell chemische (acerylcholine) als mechansche (afschuifspanning) stmulatie van uteriene arterien van wild-type en $t-A C E-$ muizen. De bijdtage van bradykinine in deze vatverwijdende responsen was groter in uteriene atterien van $\mathrm{t}-\mathrm{ACE} /$ muizen, daar farmacologische blokkade van $\mathrm{B}_{2}$ receptoren de reactie op acetylcholine en de afschuifspanning een groter effect had in $\mathrm{t}-\mathrm{ACE} /$ muizen. Tijdens de zwangerschap bleek de bijdrage van stikstofmonooxide aan de door flow-geinduceerde vatverwijing substantieler in uteriene arteriën van wild-type muzen, terwijl de bijdrage van bradykinine groter was in uteriene arteriën van hoogzwangere tACE/ muizen. Deze bevindingen tonen de modulerende rol aan van vasoactieve factoren, zoals bradykinine, geproduceerd door de endotheelcellagg gedurende de door flow-geincluceerde vatverwijding van uteriene arterièn in situaties waarbil de dootbloeding van het uteriene vaatbed chronisch is verhoogd.

Weetsel $A C E$ deficiëntie resulteerde in een verlaggde arteriele elasticiteit (hoofdstuk 6). Deze verminderde elasticiteir bleek $B_{2}$ receptor stimulatic afhankelijk te zijn, daar chronische (14 dagen) blokkade van deze receptoren de arteriele elasticiteit in $\mathrm{t}-\mathrm{ACE}$ muizen herstelde. Deze bevindingen bewijzen de interactic tussen het renine-angiotensine systeem en het kallikreine-kinine systeem en hun bijdrage aan de maturatie van het cardiowasculaire systeem.

In hoofdstuk 7 hebben we onderzocht of weefsel ACE betrokken is bij de arteriele temodeleting door de unilaterale carotide arterie ligatie methode toe te passen en utenene arterien re bestuderen vór, tijdens en na de zwangerschap. In de afgebonden carotide arieric van de weefsel ACE-deficiente mus, gladde spictel hyperplasie, imwardse remodelcring en atteriele elasticiteit waren allen veminderd in vergelijking met de carotide arterte wan de wild-type mus. Hieruit blikt dat weefsel ACE eetr grote roll vervult in het oncerdrukten van de hypeplastische inwardse remodelering wan de catotide arterie die blootgesteld is an lage afschuifsanningen. Uit de bevindingen wan hoofdstuk 3 en 7 blipt dat angiotensine II verantwoordelijk is voor de gladde spietcel hyperplasie, tenwij bradykinine de inkrimping tegengat in respons op drastische verlagingen van de doobloeding in grote arterien. Net als weetsel kallikreine bleek weefsel ACE nier essentiel voor de uitwardse hypertrofe en inwatdse hypotrofe remodelening van de uteriene artetic tijdens en na de zwangerschap.

De effecten van de uitgestelde zwangexschap van de muis op de acute en chronische uteriene artericte responsen waren het onderwerp van hoofdstuk 8 . Onze bevindingen suggereren dat op middelbate leeftijd de effecten van de afschuifspanning op de 
fosforylering wan het endotheliaal stikstofmonoxide synthase en de onderliggende stikstofmonooxide productie vefloren zijn. Verhoogde uirscheiding van vasoactieve factoren geproduceerd door het endotheel, zoals prostacycline of "endothelium-derived hyperpolarizing factor" kunnen de acute door tlow-geïnduceerde valverwijding compenseren, maar garanderen geen optimale door zwangerschap-gerelateerde remodelering van de uteriene arterie. Dit kan het verlagagde antal levensvatbare foetussen tijdens het derde trimester van de uitgestelde zwangerschap bij de muis werklaren.

Uit de resulaten beschreven in dit proefschrift blijkt dat een verlangde acute atteriële response op flow niet noodzakelijkerwijs een defect van de structurele remodelering inhoudt. Gesterkt door onze bevindingen en die van anderen, hebben we een overzichtsartikel geschreven dat de dissociatie tussen acute en chronische vaatwand responsen op flow nader belicht.

De resultaten in dic proefschrift bevestigen de gunstige arteriële effecten van bradykinine. Verder is gebleken dat acute door flow-geinduceerde vaatverwijding en chronische door flow-gerelateerde remodelering niet met elkaar geassocieerd zijn en dat hun onderliggende mechanismen via andere signaaltransductic processen verlopen. Dit biedt nieuwe perspectieven voor de ontwikkeling van waardevolle "targets" voor cardiovasculair-farmacologische therapie. Bovendien verschillen adaptieve structurele arteriële veranderingen in respons op flow tussen grote elastische vaten en kleine collaterale vaten, en het effect van zelfs een middelbare leefujd op de arteriële remodelering is uitgesprokener dan een deficiëntie van cen van de cardiovasculaire sleutel enzymen, zoals weefsel kallikreine en weefsel ACE. Dit opent nieuwe perspectieven waaronder "site-directed" interventies die leiden to een breder inzicht van de effecten van ouderdom en regionaliteit op de arteriële remodelering. 



\section{Dankwoord}

Terugkijkend op een boeiende en plezierige AlO penode, warin ik geen seconde spijt heb gehad deze stap te hebben gemakt, zijn er een antal mensen die ik will bedanken.

Mijn promotor Jo: bedankt voor de kans die je me hebt gegeren. Ik heb bewondering woor je encyclopedische kennis van de vakliteratum, die je weet te combineten net een grote creativiteit. Je gedrewenheid en passic, hetgeen zich ondemeer witte in het matendsnel contgeren van manuscripten, hebben een besmettelijk ffect gehad op mijn motivatie.

Mijn co-promotor Paul: Je vemoedde al tijdens het sollicitatiegesprek dat het goed zou komen, bedankt voor dit vertrouwen. Je praktische vaardigheden zijn indrukwekkend, vooral als je tijdens het monteren van varies in de myogtaaf de "turbo" aanzerte. We hebben er toch iets moois van weten te maken!

De kamergenoten Gregorio en Ger: Gregorio, computer-hulp-in-nood, komik, goochelaar, shngemens, genealoog, fotograaf, histoloog, .. ik kan wog wel even doorgan. Je bent een bijzonder plezierige, goedgehumeurde en vakbekwame collega. Ger, jii bent de rust zelve, en af en toe kan je heel grappig uit de hoek komen. Stille wateren hebben diepe gronden. Bovendien heb je ook een Roda hart, dus nzie zullen we nooit krigen, alhoewel dat met jou onmogelijk is. Ik zal julie collegialiteit missend

Het was een voortecht en een geweldige ervaring om een Marie Curie fellowship re krijgen voot een stage bij INSERM Unit 541 wan Bernard Levy in hartje Parijs. Daniel, 1 admire your know-how and patience. Chantal, being both a mother and scientist, thanks for all your help and hospitality. Sonis, our cooperation was frutfull, resulting in three chapters in this thesis. Good luck in New Haven. Stephanie, your laugh is contagious, thanks for making my stay in Paris a pleasant one.

De paranimfen: Henny, kersverse Doctor, je moed en je eigengereide manier om problemen an te pakken getugt wan karaker. Je begint aatig je drat te vinden in het bourgondische Zuiden. Monique, lieve zus, hierom helb ik al die (zwangere) muzen opgeofferd. Ik zal me trots en gesterkt woelen als jullie nast me stant.

Verder bedank ik mijn (ex) collega's van Jo \& Co: Jolanda, Jos en Jos, Katin, Lilian, Marijke, en de rest van de capaciteitsgroep Iarmacologie \& Toxicologic, for, je hebt me veel werk wit handen genomen met de genotypering van muizen. Paulien en de andero CPVers, reuze bedankt voor de adequate organisatie van de muzenfok De sectretaressen Els en Mr, voor hun hulp met brieven, congresbezocken en dergelijke. Trees, al zil her als secretaresse van Biochemie, toch heb je middels ic hartelijke en moederlijke persoonlijhtheid ecn steentje bijgedtagen.

Natuurlijk mag een dankbeuiging aan mijn ouders niet ontreken. Pa en ma, jullic gaven, mij alle steun, vrijheid en vertrouwen in alle keuzes die in min leven gemakt heb on 
hopelijk nog zal maken. Met bewondering en trots hebben jullie mijn AJO periode gevolgd. Van jullie heb ik geleerd dat je met wilskracht en werklust een heel eind komt in het leven. 


\section{Curriculum Vitae}

Robertus Hubertus Petronella Hilgers was bom on August $7^{\text {th }} 1972$ in Kerkrade, the Netherlands. After completing VWO-B education at the Sancta Maria College in Kerkrade (1991), he enrolled at the Hogeschool Heerlen, the Netherlands (laboratory eduction speciality biochemistry). After doing his traineeship at the Biochemistry lab of Prof. dr. Twan Sadowski at the University of British Columbia, Vancouver, Canada, he decided to continue his stay in beautiful Vancouver. Two years later he returned to the Netherlands. He worked two years in the metal industry (Chroonwerk, Kerkrade) before starting as a research analist in the lab of Prof. dr. Jas Rosing ar the Biochemistry department of the University of Maastricht (1998). In May 2000 be switched to the neighbours of the Pharmacology \& Toxicology deparment to start a PhD study in the lab of Prof. dr. Jo De Mey. During this PhD project he worked for six months with the sponsorship of a Marie Curie fellowship at the Institut National de la Santé et de la Recherche Médicale (INSERM) U541, IFR Circalation Lariboisière, Université Paris VII at the supervision of Prof. dr. Bernard Lévy, Dr. Daniel Henrion, and Dr. Chantal Boulanger in Paris, France.

Robertus Hubertus Petronelia Hilgers werd geboren op 7 augustus 1972 te Kerkrade, Nederland. Na het behalen van het VWO-B dipioma an het Sancta Mara College te Kerkrade (1991) volgde een inschrijving an de Hogeschool Heetlen, Nederland (laboratorium opleiding specialisatie). $\mathrm{Na}$ een stage in het biochemisch laboratorium watn Prof. dr. Ivan Sadowski aan de Universiteit van Brits Columbia, Vancouver, Canada, besloot hij te blijven in het mooie Vancouver. Twee jaar later keerde hij teng naar Nederland, om vervolgens twee jaar in de metaalnijverheid (Chroomwerk, Kerlirade) te werken, vooraleer hij begon als onderzoeksassistent in het lab van Prof. dr. Jan Rosing (Biochemie) van de Universiteit Maastricht (1998). In mei 2000 verthuisde hij naar de buren van Farmacologie $\&$ Toxicologie in het lab van Prof. dr. Jo De Mey om te beginnen mer het werk beschreven in dit proefschrift. Tijclens zijn promotieonderzoek heeft hij zes maanden gewerkt, met de steun van een Marie Curie Fellowship, aan het Institut National de la Santé et de la Recherche Médicale (INSERM) U541, IFR Circulation Laribotisiere, Université Paris VII onder supervisie van Prof. dr. Bernard Jévy, Dr. Daniel Henrion on Dr. Chantal Boulanger in Parijs, Frankrijk. 



\section{Grants and Awards}

Graduate Student Travel Award of the American Society for Pharmacology and Experimental Therapeutics (ASPET) at Experimental Biology "01, Orlando, Florida, March 31-April 4, 2001.

Pre-doctoral Marie Curie European Fellowship (03/2002-09/2002)

Young Investigator Award European Council for Blood Pressure and Cardiovascular Research (ECCR), Secheim, Germany, October 10-12, 2003.

\section{List of Published Articles}

Hilgers RHP, Betgaya S, Schiffers PMH, Meneton P, Boulanger CM, Henrion D, Lévy BI, De Mey JGR. Uterine Artery Structural and Functional Changes During Pregnancy in Tissue Kallikrein-Deficient Mice. Arteriosder Thromb Vasc Biol. 2003;23:1826-1832.

Aartsen WM, Hilgers RHP, Schiffers PMH, Daemen MJAP, De Mey JGR, Smits J]FM. Changes in Vascular Distensibility During Angiotensin-Converting Enzyme Inhibition Involve Bradykinin Type 2 Receptors. J V ast Res. 2004:41:18-27.

Hilgers RHP, Schiffers PMH, Aartsen WM, Fazzi GE, Smits JFM, De Mey JGR. Tissue Angiotensin-Converting Enzyme in Imposed and Physiological Flow-Related Arterial Remodeling in Mice. Artenasider Thromb Yasc Bial. In Press.

Bergaya S, Hilgers RHP, Meneton P, Dong Y, Bloch-Faure M, Inagami 'T, Alhenc-Gelas F, Levy BI, Boulanger CM. Flow-Dependent Dilatation Mediated by Endogenous Kinins Requires Angiotensin $A T_{2}$ Receptors. Cir Res. In Press

\section{List of Submitted Articles}

Bergaya S, Hilgers RHP, Schiffers PMH, Fazzi GE, Meneton P, I.évy BI, De Mey JGR, Boulanger CM. Role of Tissue Kallikrein During Arterial Remodeling in Response to Atered Blood Flow.

Hilgers RHP, Schiffers PMH, De Mey JGR. Intra-Arterial Bradykinin and Vasodilatation in Relation to Acute and Chronic Changes in Blood Flow.

Hilgers RHP, Schiffers PMH, FazzI GE, De Mey JGR. Uterinc Artery Dilataion and Remodeling During Postponed Murine Pregnancy. 
Hilgers RHP. Schiffers PMH, Wesselman JP, De Mey JGR. Acute and Chromic Adaptive Responses to Altered Blood Flow in Large and Small Arteries. (Review)

Schulten HM, Hilgers RHP, Schiftets PMH, Strzeleck A, Fazzi GE, de Muinck ED, De Mey JGR. Reduced Endothelium-Dependent Relaxations and Impaired Acute FlowInduced Vasodilatation Do Not Comptomise Chronic Flow-Related Arterial Remodeling in Hyperlipidemic Mice.

\section{List of Abstracts}

De Mey JG, Hilgers RH, Aartsen WM, Schifters PM, Smits JF. Alteted flow-induced remodeling in tissue angotensin-conwerting enzyme deficient mice. 8th Internacional Symposium on Mechanisms of Vasodiatation. May 31-June 3, 2001, Boston, Mass., USA. J Vort Rer.2001;38(suppl 1):9.

Hilgers RH, Aartsen WM, Schiffers PM, De Mey JGR. Arterial stiffness and flow-related arterial remodeling in mice during and after pregnancy. $6^{\text {th }}$ Annual Meeting of the European Council for Blood Pressute and Cardiovascular Research, Noordwijkerhout, the Netherlands, October 26-28, 2001.

Aartsen W, Hilgers R, Fazzi G, Debets J, Bitsch N, Schiffers P, Smits J, De Mey J. Phenotyping of the vasculature in mice lacking tissue bound angiotensin converting enzyme. $6^{\text {th }}$ Annual Meeting of the European Council for Blood Pressure and Carcliovascular Research, Noordwijkerhout, the Netherlands, October 26-28, 2001.

Schulten H, Hilgers RH, Schiffers PMH, Fazzi GE, De Muinck ED, De Mey JGR. Hypertrophic flow-induced arterial remodeling increases with age in hyperlipidemic mice. Fouth St-Gerlach Vascular Biology Workshop, January 24-25, 2002.

Hilgers RH, Schulten H, Schiffers PM, Fazzi GE, De Muinck E, Strzelecka A, De Mey JGR. 2002. Imparred agonist- and flow-induced endothelium-dependent responses of catotid atteries in hypercholesterolemic mice. $22^{\text {nal }}$ Meeting of the European Society for Mictocirculation, August 28-30, 2002, Unversity of Exeter, Devon, UK. I Var Res. 2002,39 (suppl 1):25.

Van der Heijen OWH, Essers YPG, Hilgers RH, Aatenburg R, Courtar DA, Kars ME, Van Breugel NHAM, Spandeman MEA, De Mey JGR, Hotker MH, Peeters LLH. Van Eys GJJM. Effect of gestation on smoothelin expression in vascular remodeling in murine uterine atery smooth muscle cells. SGI, 2003.

Hilgers $\mathbb{R}$, Bergaya S, Hennon D, Boulanger C, Meneton P, Schiffers P, De Mey JGR. Flow-induced vasodiatation and phyiological arteriogenesis. Pivotal role of tissue kallikrein in the former but not the latter. Angiogenesis Conference Leiden, February 13 15,2003 . 
Hilgers RHP, Bergaya S, Schiffers PMH, Meneton P, Boulanger CM, Henrion D, Lexy BII, De Mey JGR. Deficiency of tissue kalikitein blunts flow-induced dilatation but not remodeling in murine uterine arteries. Experimental Biology ${ }^{0} 03$, San Diego, April 11-15, 2003.

Hilgers RHP, Schiffers PMH, Aatsen WM, Smits JFM, De Mey JGR. Flow-Induced Arterial Remodeling in Tissue Angiotensin-I Converting Enzyme Deficient Mice. 8th Annual Meeting of the European Council for Blood Pressure and Cardiovascular Research, Noordwijkethout, the Netherlands, October 10-12, 2003.

Hilgers R, Bergaya S, Schiffers P, Fazzi G, Smits J, Meneton P, Boulanger C, De Mey J. Angiotensin (Ang), Bradykinin (BK) and Convening Enzyme in Imposed and Physiological Flow-Related Arterial Remodeling. IVBM Toronto, Canada, June 1-5, 2004.

Hilgers RHP, Schiffers PMH, Fazzi GE, De Mey JGR. Uterine attery dilatation and remodeling during postponed murine pregnancy. 8th International Symposium on Resistance Anteries. Angers, France, June 20-23, 2004.

Hilgers RHP, Schiffers PMH, Bergaya S, Boulanger C, De Mey JGR. Roles of agiotensin II and bradykinin in flow-related arterial remodeling in mice. $8^{\text {th }}$ International Symposium on Resistance Arteries. Angers, France, Junc 20-23, 2004. 